Supporting Information for:

\title{
Cross-Selective Aza-Pinacol Coupling via Atom Transfer Catalysis
}

Sean M. Rafferty ${ }^{\dagger}$ Joy E. Rutherford ${ }^{\dagger}$ Lumin Zhang, Lu Wang, David A. Nagib*

Department of Chemistry and Biochemistry, The Ohio State University, 151 W. Woodruff Ave., Columbus, OH, 43210, United States

\section{Corresponding Author}

*E-mail: nagib.1@osu.edu 
$\begin{array}{ll}\text { I. General information } & \text { S3 }\end{array}$

II. General procedures $\quad$ S4

III. Development of cross-selective aza-pinacol coupling S5

a. Single electron transfer (SET) strategy S5

b. Atom transfer strategy S6

c. Cross-selectivity in aza-pinacol coupling $-1: 1$ stoichiometry S8

IV. Synthesis and characterization of acyl iodide and $\alpha$ oxy iodide S9

V. Synthesis and characterization of imines $\quad$ S10

VI. Synthesis and characterization of aldehydes $\quad$ S18

VII. Synthesis and characterization of aza-pinacol couplings - imines $\quad$ S21

VIII. Synthesis and characterization of aza-pinacol - aldehydes S33

$\begin{array}{ll}\text { IX. Asymmetric aza-pinacol coupling } & \text { S39 }\end{array}$

X. Synthesis and characterization of other ketyl couplings S41

$\begin{array}{ll}\text { a. Determination of stereochemistry } & \text { S44 }\end{array}$

$\begin{array}{ll}\text { XI. Mechanistic insights } & \text { S47 }\end{array}$

$\begin{array}{ll}\text { a. Zn-mediated turnover of Mn catalyst } & \text { S47 }\end{array}$

b. Investigation of the amine role in radical termination $\quad$ S47

$\begin{array}{ll}\text { c. UV-Vis experiments } & \text { S48 }\end{array}$

$\begin{array}{ll}\text { d. Cyclic voltammetry experiments } & \text { S50 }\end{array}$

$\begin{array}{ll}\text { XII. References } & \text { S51 }\end{array}$

$\begin{array}{ll}\text { XIII. NMR Spectra } & \text { S52 }\end{array}$ 


\section{General information}

All chemicals and reagents were purchased from Sigma-Aldrich, Alfa Aesar, Acros, TCI, or ChemImpex. $\mathrm{CH}_{2} \mathrm{Cl}_{2}$, THF, $\mathrm{Et}_{2} \mathrm{O}$ and DMF were dried and degassed with nitrogen using an Innovative Technology solvent system. $\mathrm{MeCN}, \mathrm{Et}_{3} \mathrm{~N}$, and ${ }^{{ }^{1} \operatorname{Pr}_{2} \mathrm{NEt}} \mathrm{were}$ distilled over calcium hydride. SiliCycle F60 (230 - 400 mesh) silica gel was used or a CombiFlash ${ }^{\circledR}$ Automated Flash Chromatograph for flash column chromatography. Thin layer chromatography (TLC) analyses were performed using Merck silica gel 60 F254 plates and visualized under UV (254 nm), $\mathrm{KMnO}_{4}$, Ceric Ammonium Molybdate (CAM), or iodine stain. ${ }^{1} \mathrm{H},{ }^{19} \mathrm{~F},{ }^{13} \mathrm{C}$ NMR spectra were recorded using a Bruker AVIII 400 or AVIII $600 \mathrm{MHz}$ NMR spectrometer. ${ }^{1} \mathrm{H}$ NMR and ${ }^{13} \mathrm{C}$ NMR chemical shifts are reported in parts per million and referenced with respect to $\mathrm{CDCl}_{3}\left({ }^{1} \mathrm{H}\right.$ : residual $\mathrm{CHCl}_{3}$ at $\delta 7.26,{ }^{13} \mathrm{C}: \mathrm{CDCl}_{3}$ triplet at $\left.\delta 77.16\right) .{ }^{1} \mathrm{H}$ NMR data are reported as chemical shifts $(\delta \mathrm{ppm})$, multiplicity $(\mathrm{s}=$ singlet, $\mathrm{bs}=$ broad singlet, $\mathrm{d}=$ doublet, $\mathrm{t}=$ triplet, $\mathrm{q}=$ quartet, quint $=$ quintet, $\mathrm{m}$ $=$ multiplet), coupling constant $(\mathrm{Hz})$, relative integral. ${ }^{19} \mathrm{~F}$ NMR data are reported as chemical shifts $(\delta \mathrm{ppm})$. High resolution mass spectra were obtained using Bruker MicrOTOF (ESI). IR spectra were recorded using a Thermo Fisher Nicolet iS10 FT-IR and are reported in terms of frequency of absorption $\left(\mathrm{cm}^{-1}\right)$. IR spectra were recorded using a Thermo Fisher Nicolet iS10 FT-IR and are reported in terms of frequency of absorption $\left(\mathrm{cm}^{-1}\right)$ and intensity of absorption $(\mathrm{s}=$ strong, $\mathrm{m}=$ medium, $\mathrm{w}=$ weak, $\mathrm{b}=$ broad). Previously reported imines and aldehydes were characterized by ${ }^{1} \mathrm{H}$ and ${ }^{13} \mathrm{C}$ NMR and matched previous spectra. UV-Vis absorbance spectra were recorded on a Cary $5000 \mathrm{UV}-\mathrm{Vis} / \mathrm{NIR}$ spectrophotometer using an internal DRA. Cyclic voltammograms were recorded using Gamry Reference 600 Potentiostat.

Photochemical reactions were performed by stirring reactions $2 \mathrm{~cm}$ from two $23 \mathrm{~W}$ (1650 lumens) white CFL lights (GE 22847-FLE23HT3/2/10E/SW). The temperature was maintained near $23{ }^{\circ} \mathrm{C}$ by using $2-3$ fans.

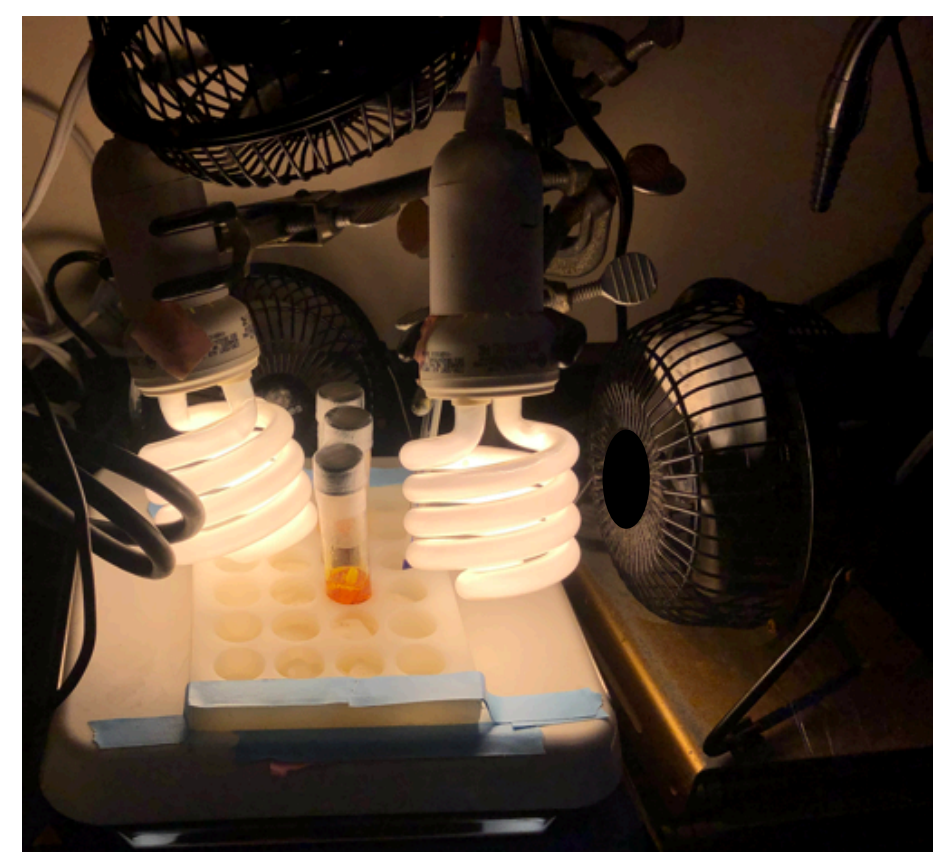

Figure S1. Two 23 W White CFL lights, with fans 


\section{General procedures}

Imine condensation - General Procedure 1 (GP1)

A round-bottom flask containing aldehyde (1-2 equiv.), $\mathrm{MsNH}_{2}$ (1 equiv.), $\mathrm{AlCl}_{3}(20 \mathrm{~mol} \%$ ), and PhMe $(0.2 \mathrm{M})$ was stirred while refluxing in a Dean-Stark apparatus for 24 hours. After cooling to room temperature, the solution was filtered through celite, then concentrated under reduced pressure. The residue was then dissolved in $\mathrm{CH}_{2} \mathrm{Cl}_{2}$ and washed with aqueous $\mathrm{NaHCO}_{3}$, which removed residual $\mathrm{MsNH}_{2}$. The organic layer was dried using $\mathrm{Na}_{2} \mathrm{SO}_{4}$ and then concentrated under reduced pressure. Recrystallization in Ethyl Acetate/Hexanes provided the pure imine.

\section{Aldehyde synthesis - General Procedure 2 (GP2)}

To a solution of oxalyl chloride (1.25 equiv.) in $\mathrm{CH}_{2} \mathrm{Cl}_{2}(0.2 \mathrm{M})$, DMSO (2.5 equiv.) in $\mathrm{CH}_{2} \mathrm{Cl}_{2}$ (2 $\mathrm{M})$ was added dropwise at $-78{ }^{\circ} \mathrm{C}$. After stirring for 30 minutes at $-78{ }^{\circ} \mathrm{C}$, alcohol (1 equiv.) in $\mathrm{CH}_{2} \mathrm{Cl}_{2}(1 \mathrm{M})$ was added dropwise. After stirring 30 minutes, $\mathrm{Et}_{3} \mathrm{~N}$ (4 equiv.) was added dropwise. The reaction mixture was transferred to an ice bath and stirred until complete, which was monitored by TLC. The reaction was quenched by the addition of $\mathrm{H}_{2} \mathrm{O}$, then extracted with $\mathrm{CH}_{2} \mathrm{Cl}_{2}$ and washed with $\mathrm{H}_{2} \mathrm{O}$ and brine.

\section{Aza-pinacol coupling using Zn - General Procedure 3 (GP3)}

To an oven dried vial of $\mathrm{CH}_{2} \mathrm{Cl}_{2}(1.0 \mathrm{~mL})$ at $0{ }^{\circ} \mathrm{C}$, AcI $(40 \mu \mathrm{L}, 0.52 \mathrm{mmol})$ was added using a 1 $\mathrm{mL}$ plastic syringe. After dropwise addition of aldehyde $(0.6 \mathrm{mmol})$, the reaction was stirred for $15 \mathrm{~min}$ at $0{ }^{\circ} \mathrm{C}$. Next, $\mathrm{Cy}_{2} \mathrm{NMe}(214 \mu \mathrm{L}, 1.0 \mathrm{mmol})$ was added at $0{ }^{\circ} \mathrm{C}$, followed by imine $(0.2$ $\mathrm{mmol}), \mathrm{Zn}(26.2 \mathrm{mg}, 0.4 \mathrm{mmol})$, and $\mathrm{Mn}_{2}(\mathrm{CO})_{10}(3.9 \mathrm{mg}, 0.01 \mathrm{mmol}, 5 \mathrm{~mol} \%)$. The reaction was degassed by stirring at $-78{ }^{\circ} \mathrm{C}$ for 20 min under vacuum. The reaction was then irradiated with white CFLs for 24 hours. The crude mixture was purified by column chromatography.

\section{Aza-pinacol coupling using Hantzsch Ester and Zn - General Procedure 4 (GP4)}

To an oven dried vial of $\mathrm{CH}_{2} \mathrm{Cl}_{2}(1.0 \mathrm{~mL})$ at $0{ }^{\circ} \mathrm{C}$, AcI $(20 \mu \mathrm{L}, 0.26 \mathrm{mmol})$ was added using a 1 $\mathrm{mL}$ plastic syringe. After dropwise addition of aldehyde $(0.3 \mathrm{mmol})$, the reaction was stirred for $15 \mathrm{~min}$ at $0{ }^{\circ} \mathrm{C}$. Next, $\mathrm{Cy}_{2} \mathrm{NMe}(86 \mu \mathrm{L}, 0.2 \mathrm{mmol})$ was added at $0{ }^{\circ} \mathrm{C}$, followed by imine $(0.2$ $\mathrm{mmol}), \mathrm{Zn}(26.2 \mathrm{mg}, 0.4 \mathrm{mmol})$, Hantzsch Ester $(38.0 \mathrm{mg}, 0.15 \mathrm{mmol})$ and $\mathrm{Mn}_{2}(\mathrm{CO})_{10}(11.6 \mathrm{mg}$, $0.03 \mathrm{mmol}, 15 \mathrm{~mol} \%$ ). The reaction mixture was degassed by stirring at $-78{ }^{\circ} \mathrm{C}$ for 20 min under vacuum and then irradiated with white CFLs for 24 hours before purification by column chromatography. 


\section{Development of cross-selective aza-pinacol coupling}

Single-electron transfer (SET) strategy:
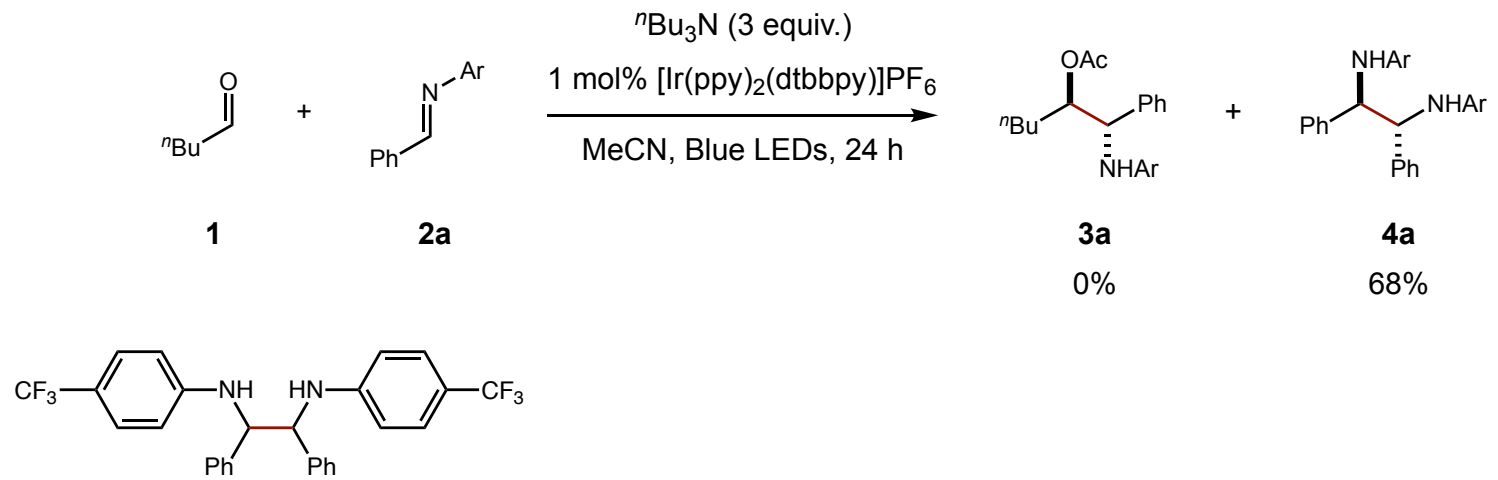

$N, N^{\prime}$-(1,2-diphenylethane-1,2-diyl)dimethanesulfonamide (4a): Following Rueping and coworker's reductive protocol ${ }^{14}$, to $\left[\operatorname{Ir}(\mathrm{ppy})_{2}(\mathrm{dtbbby})\right] \mathrm{PF}_{6}(1.8 \mathrm{mg}, 1 \mathrm{~mol} \%)$ and imine $(0.2 \mathrm{mmol}$, $49.8 \mathrm{mg}$ ) was added degassed $\mathrm{MeCN}(1.5 \mathrm{~mL})$ and ${ }^{n} \mathrm{Bu}_{3} \mathrm{~N}(143 \mu \mathrm{L}, 0.6 \mathrm{mmol})$. The reaction was irradiated under Blue LED for 24 hours. Upon completion, solvent was removed to provide $68 \%$ product 4a (1.2:1 d.r.) determined by crude NMR. $\mathrm{R}_{\mathrm{f}:} 0.41$ (15\% ethyl acetate/hexanes). ${ }^{1} \mathrm{H}$ NMR $\left(400 \mathrm{MHz}, \mathrm{CDCl}_{3}\right) \delta: 7.23-7.33(\mathrm{~m}, 10 \mathrm{H}), 6.93-6.95(\mathrm{~m}, 4 \mathrm{H}), 6.51-6.55(\mathrm{~m}, 4 \mathrm{H}), 4.98-5.00$ $(\mathrm{m}, 2 \mathrm{H}) .{ }^{13} \mathrm{C}$ NMR $\left(100 \mathrm{MHz}, \mathrm{CDCl}_{3}\right) \delta: 149.0,137.0,128.7,127.5,126.6-126.7$ (m), 124.7 (q, $\left.{ }^{1} J_{\mathrm{CF}}=262.1 \mathrm{~Hz}\right), 119.9\left(\mathrm{q},{ }^{1} J_{\mathrm{CF}}=32.6 \mathrm{~Hz}\right), 113.2,61.7 .{ }^{19} \mathrm{~F}\left(376 \mathrm{MHz}, \mathrm{CDCl}_{3}\right) \delta:-61.2,-61.3$. HRMS (ESI-TOF) m/z: calc'd for [M+H] ${ }^{+} 501.1765$, found 501.1734 . 


\section{Atom transfer strategy:}

Ir catalyzed

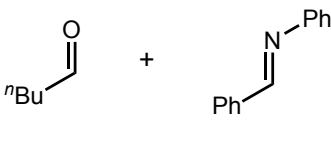

1<smiles>O=C(O)C(Br)C(Nc1ccccc1)c1ccccc1</smiles>

Acl; HE (0.75 eq), ${ }^{\mathrm{i}} \mathrm{Pr}_{2} \mathrm{NEt}$ (1 eq)

$1 \mathrm{~mol} \%\left[\operatorname{lr}(\mathrm{ppy})_{2}(\mathrm{dtbbpy}) \mathrm{PF}_{6}\right.$

$\mathrm{CH}_{2} \mathrm{Cl}_{2}$, Blue LEDs, $24 \mathrm{~h}$

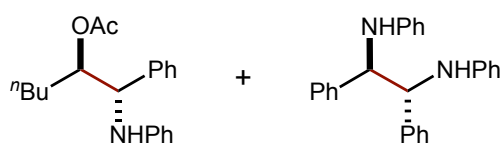

3b

$76 \%$
$4 b$

$0 \%$

1-phenyl-1-(phenylamino)hexan-2-yl acetate (3b): To an oven dried vial of $\mathrm{CH}_{2} \mathrm{Cl}_{2}(1.0 \mathrm{~mL})$ at $0{ }^{\circ} \mathrm{C}$, AcI $(50 \mu \mathrm{L}, 0.65 \mathrm{mmol})$ was added using a $1 \mathrm{~mL}$ plastic syringe. After dropwise addition of pentanal $(64 \mu \mathrm{L}, 0.6 \mathrm{mmol})$, the reaction was stirred for $15 \mathrm{~min}$ at $0{ }^{\circ} \mathrm{C}$. Next, ${ }^{i} \operatorname{Pr}_{2} \mathrm{NEt}(53 \mu \mathrm{L}, 0.3$ mmol) was added at $0{ }^{\circ} \mathrm{C}$, followed by imine ( $\left.54.3 \mathrm{mg}, 0.3 \mathrm{mmol}\right)$, Hantzsch Ester $(58.3 \mathrm{mg}, 0.23$ $\mathrm{mmol})$ and $\left[\operatorname{Ir}(\mathrm{ppy})_{2}(\mathrm{dtbbby})\right] \mathrm{PF}_{6}(2.7 \mathrm{mg}, 1 \mathrm{~mol} \%)$. The reaction was then degassed by stirring at $-78^{\circ} \mathrm{C}$ for 20 min under vacuum. The reaction was then irradiated with one $90 \mathrm{~W}$ blue LED lamp (Kessil A360WE tuna blue), then the crude mixture was purified by column chromatography to provide $76 \%$ product 3 b. $\mathrm{R}_{\mathrm{f}}: 0.51$ (15\% ethyl acetate/hexanes). ${ }^{1} \mathrm{H} \mathrm{NMR}\left(400 \mathrm{MHz}, \mathrm{CDCl}_{3}\right) \delta 7.37$ $(\mathrm{d}, J=7.4 \mathrm{~Hz}, 2 \mathrm{H}), 7.32(\mathrm{t}, J=7.6 \mathrm{~Hz}, 2 \mathrm{H}), 7.26(\mathrm{t}, J=6.7 \mathrm{~Hz}, 1 \mathrm{H}), 7.07(\mathrm{t}, J=8.0 \mathrm{~Hz}, 2 \mathrm{H}), 6.66$ $(\mathrm{t}, J=7.3 \mathrm{~Hz}, 1 \mathrm{H}), 6.50(\mathrm{~d}, J=7.7 \mathrm{~Hz}, 2 \mathrm{H}), 5.21(\mathrm{~m}, 1 \mathrm{H}), 4.55(\mathrm{~s}, 1 \mathrm{H}), 4.45(\mathrm{~s}, 1 \mathrm{H}), 2.02(\mathrm{~s}, 3 \mathrm{H})$, $1.58(\mathrm{~m}, 2 \mathrm{H}), 1.26(\mathrm{~m}, 4 \mathrm{H}), 0.84(\mathrm{t}, J=6.8 \mathrm{~Hz}, 3 \mathrm{H}) .{ }^{13} \mathrm{C} \mathrm{NMR}\left(150 \mathrm{MHz}, \mathrm{CDCl}_{3}\right) \delta 171.2,147.3$, 139.2 , 129.1, 128.5, 127.6, 127.6, 117.8, 113.8, 61.3, 29.4, 28.1, 22.4, 21.2, 14.0. HRMS (ESITOF) $\mathrm{m} / \mathrm{z}$ : calc'd for $[\mathrm{M}+\mathrm{H}]^{+} 312.1964$, found 312.1996 .

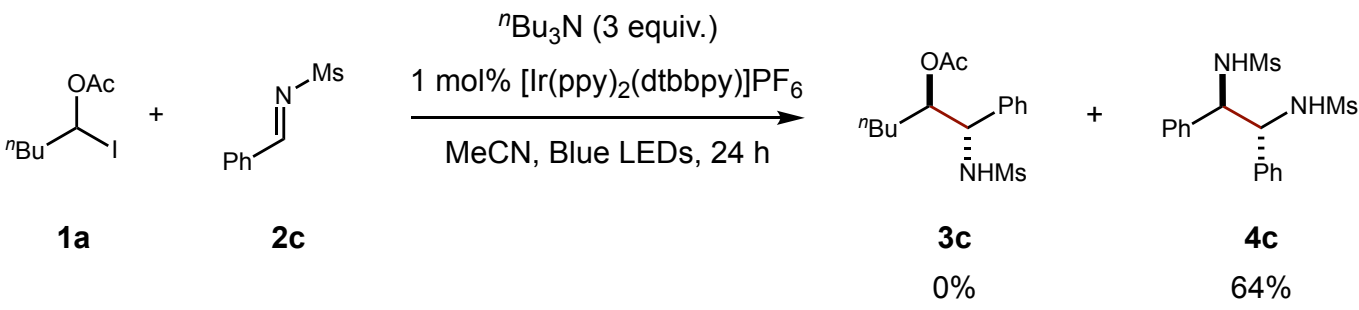

$N, N^{\prime}$-(1,2-diphenylethane-1,2-diyl)dimethanesulfonamide (4c): Following Rueping and coworker's reductive protocol ${ }^{14}$, to $\left[\operatorname{Ir}(\mathrm{ppy})_{2}(\mathrm{dtbbby})\right] \mathrm{PF}_{6}(1.8 \mathrm{mg}, 1 \mathrm{~mol} \%)$ and imine $\mathbf{S 3}(0.2$ mmol, $36.6 \mathrm{mg})$ was added degassed MeCN $(1.5 \mathrm{~mL})$, then $1 \mathrm{a}(72 \mu \mathrm{L}, 0.4 \mathrm{mmol})$ and ${ }^{n} \mathrm{Bu}_{3} \mathrm{~N}(143$ $\mu \mathrm{L}, 0.6 \mathrm{mmol})$. The reaction was irradiated under Blue LED for 24 hours. Upon completion, solvent was removed to provide $64 \%$ product $4 \mathbf{c}$ determined by crude NMR. $\mathrm{R}_{\mathrm{f}}: 0.18$ (40\% ethyl acetate/hexanes). ${ }^{1} \mathrm{H}$ NMR $\left(400 \mathrm{MHz}, \mathrm{CDCl}_{3}\right) \delta: 7.13-7.17(\mathrm{~m}, 6 \mathrm{H}), 7.01-7.04(\mathrm{~m}, 4 \mathrm{H}), 6.09$ $-6.10(\mathrm{~m}, 2 \mathrm{H}), 4.64(\mathrm{dd}, J=4.8,2.3 \mathrm{~Hz}, 2 \mathrm{H}), 2.53$ (s, 6H). ${ }^{13} \mathrm{C} \mathrm{NMR}\left(100 \mathrm{MHz}, \mathrm{CDCl}_{3}\right) \delta: 137.7$, 128.9, 128.6, 127.8, 62.5, 42.2. HRMS (ESI-TOF) $\mathrm{m} / \mathrm{z}$ : calc'd for $[\mathrm{M}+\mathrm{Na}]^{+} 391.0762$, found 391.0780 . 
Mn catalyzed

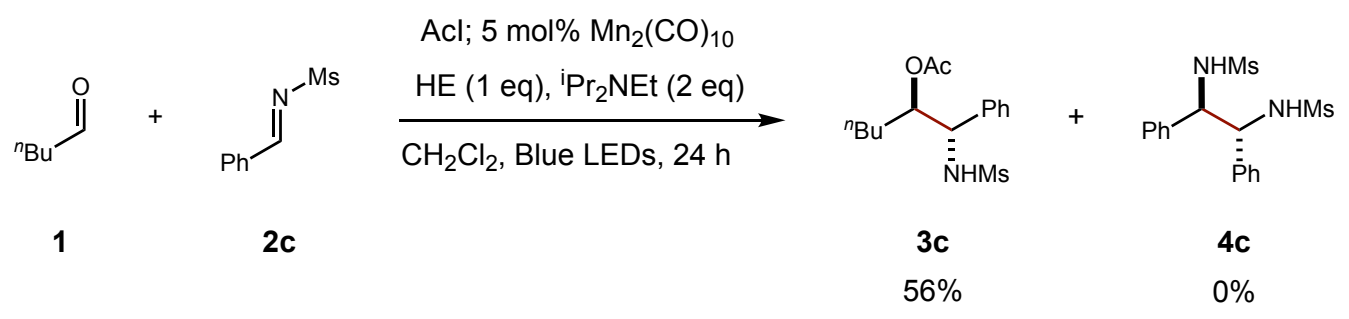

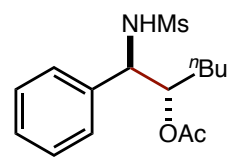

1-(methylsulfonamido)-1-phenylhexan-2-yl acetate: 1-(methylsulfonamido)-1-phenylhexan2-yl acetate (3c): To an oven dried vial of $\mathrm{CH}_{2} \mathrm{Cl}_{2}(1.0 \mathrm{~mL})$ at $0{ }^{\circ} \mathrm{C}$, AcI $(40 \mu \mathrm{L}, 0.52 \mathrm{mmol})$ was added using a $1 \mathrm{~mL}$ plastic syringe. After dropwise addition of pentanal $(0.6 \mathrm{mmol})$, the reaction was stirred for $15 \mathrm{~min}$ at $0{ }^{\circ} \mathrm{C}$. Next, ${ }^{i} \operatorname{Pr}_{2} \mathrm{NEt}(71 \mu \mathrm{L}, 0.4 \mathrm{mmol})$ was added at $0{ }^{\circ} \mathrm{C}$, followed by imine (0.2 mmol), Hantzsch Ester (50.7 mg, $0.2 \mathrm{mmol})$, and $\mathrm{Mn}_{2}(\mathrm{CO})_{10}(3.9 \mathrm{mg}, 0.01 \mathrm{mmol})$. The reaction was degassed by stirring at $-78{ }^{\circ} \mathrm{C}$ for 20 min under vacuum. The reaction was then irradiated with white CFLs for 24 hours. Upon completion, solvent was removed to provide 56\% product 3c (3:1 d.r.) determined by crude NMR. Rf: 0.43 (30\% ethyl acetate/hexanes). IR (film) $\mathrm{cm}^{-1}: 3273,3090,2957,2930,2861,1319,1231$. HRMS (ESI-TOF) m/z: calc'd for [M+Na] ${ }^{+}$ 336.1245, found 336.1269.

Major diastereomer: ${ }^{1} \mathrm{H} \mathrm{NMR}\left(400 \mathrm{MHz}, \mathrm{CDCl}_{3}\right) \delta: 7.32-7.39(\mathrm{~m}, 5 \mathrm{H}), 5.40(\mathrm{~d}, \mathrm{~J}=8.1 \mathrm{~Hz}, 1$ $\mathrm{H}), 5.11-5.14(\mathrm{~m}, 1 \mathrm{H}), 4.69(\mathrm{dd}, J=4.3,8.2 \mathrm{~Hz}, 1 \mathrm{H}), 2.62(\mathrm{~s}, 3 \mathrm{H}), 2.04(\mathrm{~s}, 3 \mathrm{H}), 1.46-1.48(\mathrm{~m}$, $2 \mathrm{H}), 1.22-1.29(\mathrm{~m}, 4 \mathrm{H}), 0.84(\mathrm{t}, J=6.8 \mathrm{~Hz}, 3 \mathrm{H}) .{ }^{13} \mathrm{C} \mathrm{NMR}\left(150 \mathrm{MHz}, \mathrm{CDCl}_{3}\right) \delta: 171.1,137.2$, $128.9,128.5,127.8,76.2,60.2,42.0,30.0,27.6,22.4,21.1,13.9$.

Minor diastereomer: ${ }^{1} \mathrm{H}$ NMR $\left(400 \mathrm{MHz} \mathrm{CDCl}_{3}\right) \delta: 7.28-7.38(\mathrm{~m}, 5 \mathrm{H}), 5.72(\mathrm{~d}, J=9.1 \mathrm{~Hz}, 1 \mathrm{H})$, 5.04-5.09 (m, 1H), $4.53(\mathrm{dd}, J=6.0,9.0 \mathrm{~Hz}, 1 \mathrm{H}), 2.55(\mathrm{~s}, 3 \mathrm{H}), 2.05(\mathrm{~s}, 3 \mathrm{H}), 1.44-1.49(\mathrm{~m}, 2 \mathrm{H})$, 1.18-1.30 (m, 4H), 0.80-0.83 (m, 3H). $\left.{ }^{13} \mathrm{C} \mathrm{NMR} \mathrm{(150} \mathrm{MHz,} \mathrm{CDCl}_{3}\right)$ 8: 171.1, 138.7, 129.2, 128.6, $127.0,76.0,60.8,42.1,31.2,27.4,22.4,21.1,14.1$. 


\section{Cross-selectivity in aza-pinacol coupling - 1:1 stoichiometry}

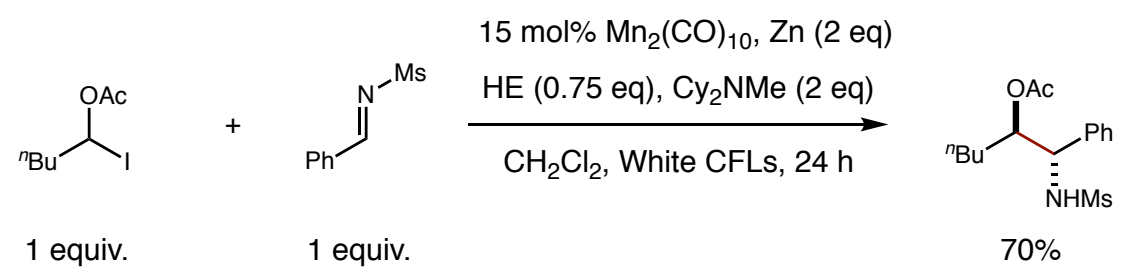

To a flame dried vial containing $\mathrm{Mn}_{2}(\mathrm{CO})_{10}(11.4 \mathrm{mg}, 0.015 \mathrm{mmol})$, imine $(0.2 \mathrm{mmol}), \mathrm{Zn}(26.2$ $\mathrm{mg}, 0.4 \mathrm{mmol}$ ), and Hantzsch Ester (38.4 mg, $0.15 \mathrm{mmol})$ in $\mathrm{CH}_{2} \mathrm{Cl}_{2}, \mathrm{Cy}_{2} \mathrm{NMe}(214 \mu \mathrm{L}, 1.0 \mathrm{mmol})$ and ${ }^{\mathrm{n}} \mathrm{BuC}(\mathrm{OAc}) \mathrm{I}(51.2 \mathrm{mg}, 0.2 \mathrm{mmol})$ was added. The reaction was then degassed by stirring at $78{ }^{\circ} \mathrm{C}$ under vacuum for 30 minutes. The reaction was irradiated with white CFL lights for 24 hours. The reaction mixture was passed through a silica plug using $10 \%$ ethyl acetate/hexanes (50 $\mathrm{mL})$ to remove residual $\mathrm{Mn}_{2}(\mathrm{CO})_{10}$. EtOAc $(50 \mathrm{~mL})$ was passed through the silica and the reaction mixture was concentrated to yield $70 \%$ product $3 \mathbf{c}$ (3:1), which was determined by using $\mathrm{CH}_{2} \mathrm{Br}_{2}$ $(7 \mu \mathrm{L}, 0.1 \mathrm{mmol})$ as an internal standard.

$\begin{array}{ccccc}\text { entry } & \text { Acl:Aldehyde:Imine (Equiv) } & \text { Hantzsch Ester } & \mathrm{Cy}_{2} \mathrm{NMe}(\text { Equiv) } & \text { yield (\%), d.r. } \\ 1 & 2.6: 3: 1 & - & 5 \text { equiv } & 90 \%, 3: 1 \\ 2 & 1.6: 2: 1 & - & 5 \text { equiv } & 62 \%, 3: 1 \\ 3 & 1.3: 1.5: 1 & + & 2 \text { equiv } & 73 \%, 3: 1 \\ 4 & 1: 1^{*} & + & 2 \text { equiv } & 70 \%, 3: 1\end{array}$

Table S1: Investigation of equivalency of AcI to aldehyde to imine using GP1 and GP2 conditions. ${ }^{*}$ using 1 equivalent of $\alpha$ oxy iodide instead of in-situ activation of aldehyde. 


\section{Synthesis and characterization of acyl iodide and $\alpha$-oxy iodide}<smiles>CC(=O)I</smiles>

acetyl iodide (S1): To flame dried $\mathrm{NaI}(23.7 \mathrm{~g}, 158 \mathrm{mmol})$, add $\mathrm{AcCl}(10.6 \mathrm{~mL}, 150 \mathrm{mmol})$ dropwise at $0{ }^{\circ} \mathrm{C}$. The reaction was warmed to room temperature and stirred for 2 hours. The mixture was then filtered to remove salts. The crude product was distilled at $140{ }^{\circ} \mathrm{C}$ at 1 atm to afford the product as yellow to light red liquid. The product was then stored in the freezer. ${ }^{1} \mathrm{H}$ NMR (600 MHz, $\left.\mathrm{CDCl}_{3}\right) \delta: 2.97$ (s, $\left.3 \mathrm{H}\right)$.<smiles>[13CH3]C([18OH])I</smiles>

1-iodopentyl acetate (1a): To an oven dried vial with AcI $(0.83 \mathrm{~mL}, 11 \mathrm{mmol})$, pentanal $(1.06$ $\mathrm{mL}, 10 \mathrm{mmol}$ ) was added at $0{ }^{\circ} \mathrm{C}$. The reaction was allowed to stir for $20 \mathrm{~min}$ at $0{ }^{\circ} \mathrm{C}$. The reaction was diluted with $\mathrm{CH}_{2} \mathrm{Cl}_{2}$, then transferred to a separatory funnel and quenched with aq. $\mathrm{NaHCO}_{3}$ and aq. $\mathrm{Na}_{2} \mathrm{~S}_{2} \mathrm{O}_{3}$, then extracted with $\mathrm{CH}_{2} \mathrm{Cl}_{2}$. The organic layer was dried over $\mathrm{Na}_{2} \mathrm{SO}_{4}$, then filtered over silica to a flask wrapped in aluminum foil. Solvent was removed at reduced pressure at room temperature and the pure $\alpha$ oxy iodide was obtained as a yellow oil. ${ }^{1} \mathrm{H} \mathrm{NMR} \mathrm{(400} \mathrm{MHz}$, $\left.\mathrm{CDCl}_{3}\right) \delta: 6.78(\mathrm{t}, \mathrm{J}=6.1 \mathrm{~Hz}, 1 \mathrm{H}), 2.11-2.17(\mathrm{~m}, 2 \mathrm{H}), 2.06(\mathrm{~s}, 3 \mathrm{H}), 1.32-1.43(\mathrm{~m}, 4 \mathrm{H}), 0.91$ $(\mathrm{t}, \mathrm{J}=7.1 \mathrm{~Hz}, 3 \mathrm{H}) .{ }^{13} \mathrm{C} \mathrm{NMR}\left(150 \mathrm{MHz}, \mathrm{CDCl}_{3}\right) \delta: 168.6,55.9,41.7,29.7,21.7,21.3,14.0$.

*Stored in freezer, slowly turns dark red over time. 


\section{Synthesis and characterization of imines}<smiles>CS(=O)(=O)c1ccccc1</smiles>

$N$-benzylidenemethanesulfonamide (2c): Benzaldehyde (1.5 equiv, $3.05 \mathrm{~mL}, 30 \mathrm{mmol})$ was subjected to GP1. Recrystallization from ethyl acetate and hexanes provided the product $(2.71 \mathrm{~g}$, 74\%) as a white solid. ${ }^{1} \mathrm{H}$ NMR $\left(600 \mathrm{MHz}, \mathrm{CDCl}_{3}\right) \delta: 9.04(\mathrm{~s}, 1 \mathrm{H}), 7.96(\mathrm{~d}, J=8.1 \mathrm{~Hz}, 2 \mathrm{H}), 7.66$ $(\mathrm{t}, J=7.5 \mathrm{~Hz}, 1 \mathrm{H}), 7.54(\mathrm{t}, J=7.8 \mathrm{~Hz}), 3.14(\mathrm{~s}, 3 \mathrm{H}) .{ }^{13} \mathrm{C} \mathrm{NMR}\left(150 \mathrm{MHz}, \mathrm{CDCl}_{3}\right) \delta: 171.8,135.3$, $132.3,131.4,129.4,40.4$. Spectral data matched those previously reported ${ }^{1}$.<smiles>CC(C)(F)c1ccc(C=[W])cc1</smiles>

N-(4-(trifluoromethyl)benzylidene)methanesulfonamide (S5): 4-trifluoromethylbenzaldehyde (1.4 eq, $1.91 \mathrm{~mL}, 14 \mathrm{mmol}$ ) was subjected to GP1. Recrystallization from ethyl acetate and hexanes provided the product $(1.68 \mathrm{~g}, 67 \%) .{ }^{1} \mathrm{H} \mathrm{NMR}\left(400 \mathrm{MHz}, \mathrm{CDCl}_{3}\right) \delta: 9.09(\mathrm{~s}, 1 \mathrm{H}), 8.09(\mathrm{~d}, J=8.1$ $\mathrm{Hz}, 2 \mathrm{H}), 7.81(\mathrm{~d}, J=8.2 \mathrm{~Hz}, 2 \mathrm{H}), 3.17(\mathrm{~s}, 3 \mathrm{H}) ;{ }^{13} \mathrm{C} \mathrm{NMR}\left(150 \mathrm{MHz}, \mathrm{CDCl}_{3}\right) \delta: 170.2,136.1$ (q, $\left.{ }^{2} J_{\mathrm{CF}}=32.8 \mathrm{~Hz}\right), 135.2,131.5,126.3\left(\mathrm{q},{ }^{4} J_{\mathrm{CF}}=3.7 \mathrm{~Hz}\right), 123.4\left(\mathrm{~d},{ }^{1} J_{\mathrm{CF}}=273 \mathrm{~Hz}\right), 40.4 .{ }^{19} \mathrm{~F} \mathrm{NMR}$ $\delta$ : $-63.34\left(377 \mathrm{MHz}, \mathrm{CDCl}_{3}\right)$. Spectral data matched those previously reported. ${ }^{2}$<smiles>CN(C)c1ccc(Br)cc1</smiles>

$\boldsymbol{N}$-(4-bromobenzylidene)methanesulfonamide (S6): 4-bromobenzaldehyde (1.4 eq, 2.59 g, 14 mmol) was subjected to GP1. Recrystallization from ethyl acetate and hexanes provided the product (0.95 g, 36\%). ${ }^{1} \mathrm{H}$ NMR (400 MHz, $\left.\mathrm{CDCl}_{3}\right) \delta: 8.99(\mathrm{~s}, 1 \mathrm{H}), 7.81-7.83(\mathrm{~m}, 2 \mathrm{H}), 7.67-7.70$ $(\mathrm{m}, 2 \mathrm{H}), 3.14(\mathrm{~s}, 3 \mathrm{H}) .{ }^{13} \mathrm{C} \mathrm{NMR}\left(150 \mathrm{MHz} \mathrm{CDCl}_{3}\right) \delta: 170.5,132.9,132.5,131.0,130.7,40.4$. Spectral data matched those previously reported. ${ }^{3}$ 
<smiles>Cc1ccc(C=[N+]([O-])c2ccccc2)cc1</smiles>

$\mathrm{N}$-(4-methylbenzylidene)methanesulfonamide (S7): 4-methylbenzaldehyde (1.4 eq, $2.36 \mathrm{~mL}$, $20 \mathrm{mmol}$ ) was subjected to GP1. Recrystallization from ethyl acetate and hexanes provided the product (1.25 g, 33\%). ${ }^{1} \mathrm{H} \mathrm{NMR}\left(400 \mathrm{MHz}, \mathrm{CDCl}_{3}\right) \delta: 8.99(\mathrm{~s}, 1 \mathrm{H}), 7.85(\mathrm{~d}, J=8.2 \mathrm{~Hz}, 2 \mathrm{H}), 7.34$ $(\mathrm{d}, J=8.0 \mathrm{~Hz}, 2 \mathrm{H}), 3.12(\mathrm{~s}, 3 \mathrm{H}), 2.46(\mathrm{~s}, 3 \mathrm{H}) .{ }^{13} \mathrm{C} \mathrm{NMR}\left(150 \mathrm{MHz}, \mathrm{CDCl}_{3}\right) \delta: 146.8,131.5,130.2$, $129.7,40.5,22.1$. Spectral data matched those previously reported. ${ }^{2}$<smiles></smiles>

$N$-(4-methoxybenzylidene)methanesulfonamide (S8): 4-methoxybenzaldehyde (1.4 eq, $1.7 \mathrm{~mL}$, $14 \mathrm{mmol}$ ) was subjected to GP1. Recrystallization from ethyl acetate and hexanes provided the product (0.82 g, 39\%). ${ }^{1} \mathrm{H}$ NMR (400 MHz, $\left.\mathrm{CDCl}_{3}\right) \delta: 8.93(\mathrm{~s}, 1 \mathrm{H}), 7.91-7.93(\mathrm{~m}, 2 \mathrm{H}), 7.00-7.02$ $(\mathrm{m}, 2 \mathrm{H}), 3.91(\mathrm{~s}, 3 \mathrm{H}), 3.11(\mathrm{~s}, 3 \mathrm{H}) .{ }^{13} \mathrm{C} \mathrm{NMR}\left(150 \mathrm{MHz}, \mathrm{CDCl}_{3}\right) \delta: 170.6,165.6,133.8,125.0$, $114.9,55.8,40.5$. Spectral data matched those previously reported. ${ }^{4}$<smiles>O=Cc1ccccc1F</smiles>

$\boldsymbol{N}$-(2-fluorobenzylidene)methanesulfonamide (S9): 2-fluorobenzaldehyde (1.4 eq, $0.59 \mathrm{~mL}, 5.6$ $\mathrm{mmol}$ ) was subjected to GP1. Recrystallization from ethyl acetate and hexanes provided the product $(0.4 \mathrm{~g}, 50 \%) .{ }^{1} \mathrm{H}$ NMR $\left(400 \mathrm{MHz} \mathrm{CDCl}_{3}\right) \delta: 9.38(\mathrm{~s}, 1 \mathrm{H}), 8.10-8.14(\mathrm{~m}, 1 \mathrm{H}), 7.63-7.68$ $(\mathrm{m}, 1 \mathrm{H}), 7.27-7.32(\mathrm{~m}, 1 \mathrm{H}), 7.18-7.23(\mathrm{~m}, 1 \mathrm{H}), 3.15(\mathrm{~s}, 3 \mathrm{H}) .{ }^{13} \mathrm{C} \mathrm{NMR}\left(150 \mathrm{MHz}, \mathrm{CDCl}_{3}\right) \delta: 165.4$ $(\mathrm{d}, J=6.0 \mathrm{~Hz}), 163.7,137.4(\mathrm{~d}, J=8.8 \mathrm{~Hz}), 129.4,125.1(\mathrm{~d}, J=3.3 \mathrm{~Hz}), 120.4(\mathrm{~d}, J=8.8 \mathrm{~Hz})$, $116.7(\mathrm{~d}, J=20.9 \mathrm{~Hz}), 40.3 .{ }^{19} \mathrm{~F} \mathrm{NMR} \delta$ : -115.67 (377 MHz, $\left.\mathrm{CDCl}_{3}\right)$. Spectral data matched those previously reported. ${ }^{5}$<smiles>O=[N+]([O-])c1ccccc1Br</smiles>

$N$-(2-bromobenzylidene)methanesulfonamide (S10): 2-bromobenzaldehyde (1.5 eq, $1.75 \mathrm{~mL}$, $15 \mathrm{mmol}$ ) was subjected to GP1. Recrystallization from ethyl acetate and hexanes provided the product (1.14 g, 45\%). ${ }^{1} \mathrm{H}$ NMR (400 $\left.\mathrm{MHz}, \mathrm{CDCl}_{3}\right) \delta: 9.48(\mathrm{~s}, 1 \mathrm{H}), 8.19-8.22(\mathrm{~m}, 1 \mathrm{H}), 7.69-7.71$ $(\mathrm{m}, 1 \mathrm{H}), 7.42-7.51(\mathrm{~m}, 2 \mathrm{H}), 3.16(\mathrm{~s}, 3 \mathrm{H}) .{ }^{13} \mathrm{C} \mathrm{NMR}\left(150 \mathrm{MHz}, \mathrm{CDCl}_{3}\right) \delta: 171.0,136.1,134.1$, $131.1,130.6,129.3,128.1,40.4$. Spectral data matched those previously reported. ${ }^{2}$ 
Me

$\mathrm{N}$-(2-methylbenzylidene)methanesulfonamide (S11): 2-methylbenzaldehyde (1.5 equiv, 1.73 $\mathrm{mL}, 15 \mathrm{mmol}$ ) was subjected to GP1. Recrystallization from ethyl acetate and hexanes provided the product $(1.35 \mathrm{~g}, 72 \%)$ as a white solid. ${ }^{1} \mathrm{H}$ NMR $\left(600 \mathrm{MHz}, \mathrm{CDCl}_{3}\right) \delta: 9.33(\mathrm{~s}, 1 \mathrm{H}), 8.05(\mathrm{~d}, J$ $=7.9 \mathrm{~Hz}, 1 \mathrm{H}), 7.51(\mathrm{t}, J=7.6 \mathrm{~Hz}, 1 \mathrm{H}), 7.33(\mathrm{t}, J=7.5 \mathrm{~Hz}, 1 \mathrm{H}), 7.30(\mathrm{~d}, J=7.7 \mathrm{~Hz}, 1 \mathrm{H}), 3.14$ $(\mathrm{s}, 3 \mathrm{H}), 2.63(\mathrm{~s}, 3 \mathrm{H}) .{ }^{13} \mathrm{C} \mathrm{NMR}\left(150 \mathrm{MHz}, \mathrm{CDCl}_{3}\right) \delta: 170.3,142.6,135.0,131.8,130.6,130.2$, $126.8,40.5,19.8$. Spectral data matched those previously reported. ${ }^{6}$<smiles>CN(C)c1cccc(Br)c1</smiles>

$\boldsymbol{N}$-(3-bromobenzylidene)methanesulfonamide (S12): 3-bromobenzaldehyde (2 eq, $2.33 \mathrm{~mL}, 20$ mmol) was subjected to GP1. Recrystallization from ethyl acetate and hexanes provided the product $(2 \mathrm{~g}, 78 \%) .{ }^{1} \mathrm{H}$ NMR $\left(400 \mathrm{MHz}, \mathrm{CDCl}_{3}\right) \delta: 8.97(\mathrm{~s}, 1 \mathrm{H}), 8.15(\mathrm{t}, J=1.8 \mathrm{~Hz}, 1 \mathrm{H}), 7.84-$ $7.87(\mathrm{~m}, 1 \mathrm{H}), 7.76-7.79(\mathrm{~m}, 1 \mathrm{H}), 7.42(\mathrm{t}, J=7.9 \mathrm{~Hz}, 1 \mathrm{H}), 3.15(\mathrm{~s}, 3 \mathrm{H}) .{ }^{13} \mathrm{C} \mathrm{NMR}(150 \mathrm{MHz}$, $\left.\mathrm{CDCl}_{3}\right) \delta: 170.2,138.0,134.1,133.4,130.8,130.3,123.6,40.4$. Spectral data matched those previously reported. ${ }^{4}$<smiles>Cc1cccc(C=[N+]([O-])c2ccccc2)c1</smiles>

$N$-(3-methylbenzylidene)methanesulfonamide (S13): 3-methylbenzaldehyde (1.4 eq, $0.67 \mathrm{~g}, 5.6$ mmol) was subjected to GP1. Recrystallization from ethyl acetate and hexanes provided the product (0.65 g, 82\%). ${ }^{1} \mathrm{H}$ NMR (400 $\mathrm{MHz}^{\mathrm{CDCl}} 3$ ) $\delta: 9.00(\mathrm{~s}, 1 \mathrm{H}), 7.79(\mathrm{~s}, 1 \mathrm{H}), 7.74(\mathrm{~d}, J=7.5$ $\mathrm{Hz}, 1 \mathrm{H}), 7.39-7.48$ (m, 2H), 3.13 (s, 3H), 2.43 (s, 3H). ${ }^{13} \mathrm{C}$ NMR (150 MHz, $\left.\mathrm{CDCl}_{3}\right) \delta: 171.9$, 139.3, 132.2, 131.5, 129.2, 129.1, 40.4, 21.3. Spectral data matched those previously reported. ${ }^{6}$ 
<smiles>O=[W]c1cccc(Oc2ccccc2)c1</smiles>

$\boldsymbol{N}$-(3-(benzyloxy)benzylidene)methanesulfonamide (S14): 3-benzyloxybenzaldehyde (1.2 eq, $0.3 \mathrm{~g}, 1.44 \mathrm{mmol}$ ) was subjected to GP1. Recrystallization from ethyl acetate and hexanes provided the product $(0.79 \mathrm{~g}, 38 \%)$. Rf: 0.50 (40\% Ethyl acetate/hexanes). ${ }^{1} \mathrm{H}$ NMR $\left(400 \mathrm{MHz}, \mathrm{CDCl}_{3}\right) \delta$ : $8.99(\mathrm{~s}, 1 \mathrm{H}), 7.26-7.60(\mathrm{~m}, 9 \mathrm{H}), 5.13(\mathrm{~s}, 2 \mathrm{H}), 3.13(\mathrm{~s}, 3 \mathrm{H}) .{ }^{13} \mathrm{C} \mathrm{NMR}\left(150 \mathrm{MHz}, \mathrm{CDCl}_{3}\right) \delta: 171.6$, 159.3, 136.2, 133.4, 130.3, 128.7, 128.3, 127.6, 125.4, 123.0, 114.7, 70.3, 40.3. IR (film): 3012, 1582, 1443, 1296, 1275, 1261, 1137, 961, 809, 799, 784, 750, 697, 680, 505, 452. HRMS (ESITOF) $\mathrm{m} / \mathrm{z}$ : calc'd for $[\mathrm{M}+\mathrm{Na}]^{+} 312.0670$, found 312.0828 .<smiles>Cc1ccc(F)cc1C=[N+]([O-])O</smiles>

$\mathrm{N}$-(5-fluoro-2-methylbenzylidene)methanesulfonamide (S15): 5-fluoro-2-methylbenzaldehyde ( $1 \mathrm{~g}, 7.23 \mathrm{mmol}$ ) was subjected to GP1. Recrystallization from ethyl acetate and hexanes provided the product (1.1 g, 72\%) as a white solid. $\mathrm{R}_{\mathrm{f}}$ : 0.55 (15\% Ethyl acetate/hexanes). ${ }^{1} \mathrm{H}$ NMR (400 $\left.\mathrm{MHz}, \mathrm{CDCl}_{3}\right) \delta: 9.30(\mathrm{~d}, J=2.1 \mathrm{~Hz}, 1 \mathrm{H}), 7.78(\mathrm{dd}, J=2.7,9.2 \mathrm{~Hz}, 1 \mathrm{H}), 7.20-7.29(\mathrm{~m}, 2 \mathrm{H}), 3.15$ $(\mathrm{s}, 3 \mathrm{H}), 2.58(\mathrm{~s}, 3 \mathrm{H}) \cdot{ }^{13} \mathrm{C} \mathrm{NMR}\left(100 \mathrm{MHz}, \mathrm{CDCl}_{3}\right) \delta: 168.8\left(\mathrm{~d},{ }^{4} J_{\mathrm{CF}}=2.6 \mathrm{~Hz}\right), 161.3\left(\mathrm{~d},{ }^{1} J_{\mathrm{CF}}=\right.$ $246.9 \mathrm{~Hz}) 138.3\left(\mathrm{~d},{ }^{4} J_{\mathrm{CF}}=3.1 \mathrm{~Hz}\right), 133.3\left(\mathrm{~d},{ }^{3} J_{\mathrm{CF}}=7.3 \mathrm{~Hz}\right), 131.7\left(\mathrm{~d},{ }^{3} J_{\mathrm{CF}}=7.3 \mathrm{~Hz}\right), 122.1(\mathrm{~d}$, $\left.{ }^{2} J_{\mathrm{CF}}=21.4 \mathrm{~Hz}\right), 115.7\left(\mathrm{q},{ }^{2} J_{\mathrm{CF}}=22.6 \mathrm{~Hz}\right), 40.4,18.7 .{ }^{19} \mathrm{~F}\left(376 \mathrm{MHz}, \mathrm{CDCl}_{3}\right) \delta:-115.1 . \mathrm{HRMS}$ (ESI-TOF) $\mathrm{m} / \mathrm{z}$ : calc'd for $[\mathrm{M}+\mathrm{Na}]^{+} 238.0314$, found 238.0318 .<smiles>CN(C)c1cc(C(F)(F)F)cc(C(F)(F)F)c1</smiles>

$\mathbf{N}$-(3,5-bis(trifluoromethyl)benzylidene) methanesulfonamide (S16): 3,5-bis(trifluoromethyl) benzaldehyde ( 1.5 equiv, $4.94 \mathrm{~mL}, 30 \mathrm{mmol}$ ) was subjected to GP1. Recrystallization from ethyl acetate and hexanes provided the product $(2.28 \mathrm{~g}, 72 \%)$ as a white solid. $\mathrm{R}_{\mathrm{f}}$ : 0.15 (15\% Ethyl acetate/hexanes). ${ }^{1} \mathrm{H}$ NMR $\left(600 \mathrm{MHz}, \mathrm{CDCl}_{3}\right) \delta: 9.15(\mathrm{~s}, 1 \mathrm{H}), 8.42,(\mathrm{~s}, 2 \mathrm{H}), 8.15(\mathrm{~s}, 1 \mathrm{H}), 3.21(\mathrm{~s}$, $3 \mathrm{H}) .{ }^{13} \mathrm{C}$ NMR $\left(150 \mathrm{MHz}, \mathrm{CDCl}_{3}\right) \delta: 168.6,134.2,133.3\left(\mathrm{q},{ }^{2} J_{\mathrm{CF}}=34.4 \mathrm{~Hz}\right) 130.8\left(\mathrm{q},{ }^{3} J_{\mathrm{CF}}=3.7\right.$ $\mathrm{Hz}), 127.9-128.1(\mathrm{~m}), 122.7\left(\mathrm{q},{ }^{1} J_{\mathrm{CF}}=273.1 \mathrm{~Hz}\right), 40.4 .{ }^{19} \mathrm{~F}\left(376 \mathrm{MHz}, \mathrm{CDCl}_{3}\right) \delta:-63.1 . \mathrm{HRMS}$ (ESI-TOF) $\mathrm{m} / \mathrm{z}$ : calc'd for $[\mathrm{M}+\mathrm{Na}]^{+} 341.9999$, found 342.0007 . 
<smiles>CN(C)c1ccc(F)nc1F</smiles>

N-((2,6-difluoropyridin-3-yl)methylene)methanesulfonamide (S17): 2,6-difluoronicotinaldehyde (1 g, $7.0 \mathrm{mmol})$ was subjected to GP1. Recrystallization from ethyl acetate and hexanes provided (937 mg, 57\%) as a white solid. $\mathrm{R}_{\mathrm{f}}$ : 0.42 (50\% Ethyl acetate/hexanes). ${ }^{1} \mathrm{H}$ NMR (400 $\left.\mathrm{MHz}_{\mathrm{CDCl}}\right) \delta: 9.25(\mathrm{~s}, 1 \mathrm{H}), 8.66(\mathrm{dd}, J=8.6,16.1 \mathrm{~Hz}, 1 \mathrm{H}), 7.03(\mathrm{dd}, J=2.8,8.3 \mathrm{~Hz}, 1 \mathrm{H}), 3.16$ $(\mathrm{s}, 3 \mathrm{H}) .{ }^{13} \mathrm{C} \mathrm{NMR}\left(100 \mathrm{MHz}, \mathrm{CDCl}_{3}\right) \delta: 165.5\left(\mathrm{dd},{ }^{1,3} J_{\mathrm{CF}}=15.2,258.1 \mathrm{~Hz}\right), 162.9\left(\mathrm{dd},{ }^{1,3} J_{\mathrm{CF}}=15.8\right.$, $257.7 \mathrm{~Hz}) 144.8\left(\mathrm{dd},{ }^{3,3} J_{\mathrm{CF}}=2.0,9.7 \mathrm{~Hz}\right), 112.7\left(\mathrm{dd},{ }^{2,4} J_{\mathrm{CF}}=5.5,28.1 \mathrm{~Hz}\right), 108.5\left(\mathrm{dd},{ }^{2,4} J_{\mathrm{CF}}=5.5\right.$, $34.8 \mathrm{~Hz}), 40.3 .{ }^{19} \mathrm{~F}\left(376 \mathrm{MHz}, \mathrm{CDCl}_{3}\right) \delta:-68.5,55.4$. HRMS (ESI-TOF) $\mathrm{m} / \mathrm{z}$ : calc'd for $[\mathrm{M}+\mathrm{Na}]^{+}$ 243.0016, found 243.0010 .<smiles>[N+]=CCCCc1ccccc1</smiles>

4-methyl-N-(4-phenylbutylidene)benzenesulfonamide (S18): To a round bottom flask with a stir bar, sodium 4-methylbenzenesulfinate (1 eq, $0.89 \mathrm{~g}, 5 \mathrm{mmol}$ ), toluene-4-sulfonamide ( $1 \mathrm{eq}$, $0.85 \mathrm{~g}, 5 \mathrm{mmol})$, pentanal (1 eq, $0.53 \mathrm{~mL}, 5 \mathrm{mmol})$, formic acid $(9 \mathrm{~mL})$, and water $(9 \mathrm{~mL})$ were added. The mixture was stirred at room temperature for 16 hours. The solid was filtered and washed with distilled water, then pentanes. The precipitate was dissolved in $\mathrm{CH}_{2} \mathrm{Cl}_{2}$ and stirred with saturated aqueous $\mathrm{NaHCO}_{3}$ for 2 hours. The combined organic layer was extracted with $\mathrm{CH}_{2} \mathrm{Cl}_{2}$ and dried over $\mathrm{NaHCO}_{3}$. The reaction mixture was then filtered and concentrated under reduced pressure to yield product $(0.52 \mathrm{~g}, 70 \%)$. $\mathrm{R}_{\mathrm{f}}: 0.6(30 \%$ Ethyl acetate/hexanes $)$. ${ }^{1} \mathrm{H}$ NMR $\left(400 \mathrm{MHz}, \mathrm{CDCl}_{3}\right) \delta: 8.59(\mathrm{t}, J=4.3 \mathrm{~Hz}, 1 \mathrm{H}), 7.79-7.82(\mathrm{~m}, 2 \mathrm{H}), 7.31-7.35(\mathrm{~m}, 2 \mathrm{H}), 7.24-7.28$ (m, 2H), 7.16-7.20 (m, 1H), 7.10-7.12 (m, 2H), $2.64(\mathrm{t}, J=7.5 \mathrm{~Hz}, 2 \mathrm{H}), 2.50-2.54(\mathrm{~m}, 2 \mathrm{H}), 2.43$ $(\mathrm{s}, 3 \mathrm{H}), 1.95$ (q, $J=7.5 \mathrm{~Hz}, 2 \mathrm{H}) .{ }^{13} \mathrm{C} \mathrm{NMR}\left(150 \mathrm{MHz}, \mathrm{CDCl}_{3}\right) \delta: 177.9,144.8,140.9,134.7$, 129.9, 128.6, 128.5, 128.2, 126.3, 35.5, 35.0, 26.2, 21.7. IR (neat): 3026, 2924, 1626, 1596, 1495, 1452, 1320, 1290, 1155, 1089, 812, 733, 699, 620, 549. HRMS (ESI-TOF) m/z: calc'd for $[\mathrm{M}+\mathrm{Na}]^{+}$302.1136, found 302.1162.

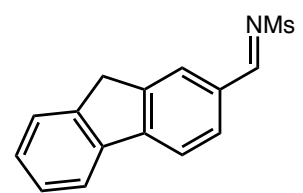

$\mathrm{N}$-((9H-fluoren-2-yl)methylene)methanesulfonamide (S19): 9H-fluorene-2-carbaldehyde (1.9 g, $10 \mathrm{mmol})$ was subjected to GP1. Column chromatography (10\% Ethyl Acetate/Hexanes) provided the product $(1.0 \mathrm{~g}, 25 \%)$ as an off-white solid. $\mathrm{R}_{\mathrm{f}}: 0.13(15 \%$ Ethyl acetate/hexanes $) .{ }^{1} \mathrm{H}$ NMR (400 MHz, $\left.\mathrm{CDCl}_{3}\right) \delta: 9.09(\mathrm{~s}, 1 \mathrm{H}), 8.17(\mathrm{~s}, 1 \mathrm{H}), 7.87-7.98(\mathrm{~m}, 3 \mathrm{H}), 7.60-7.63(\mathrm{~m}, 1 \mathrm{H})$, $7.40-7.47(\mathrm{~m}, 2 \mathrm{H}), 3.99(\mathrm{~s}, 2 \mathrm{H}), 3.16(\mathrm{~s}, 3 \mathrm{H}) .{ }^{13} \mathrm{C} \mathrm{NMR}\left(100 \mathrm{MHz}, \mathrm{CDCl}_{3}\right) \delta: 171.8,149.1$, $144.9,144.1,140.2$, 131.6, 130.6, 129.0, 127.4, 127.4, 125.5, 121.4, 120.6, 40.6, 36.9. HRMS (ESI-TOF) m/z: calc'd for [M+Na] ${ }^{+} 294.0565$, found 294.0588. 


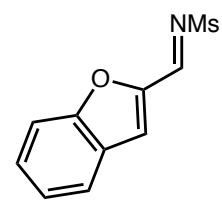

$\mathrm{N}$-(benzofuran-2-ylmethylene)methanesulfonamide (S20): Benzofuran-2-carbaldehyde (1.4 eq, $1.7 \mathrm{~mL}, 14 \mathrm{mmol}$ ) was subjected to GP1. Recrystallization from ethyl acetate and hexanes provided the product (1.2 g, 54\%). $\mathrm{R}_{\mathrm{f}}: 0.38$ (40\% Ethyl acetate/hexanes). ${ }^{1} \mathrm{H} \mathrm{NMR}(400 \mathrm{MHz}$, $\left.\mathrm{CDCl}_{3}\right) \delta: 8.95(\mathrm{~s}, 1 \mathrm{H}), 7.74-7.76(\mathrm{~m}, 1 \mathrm{H}), 7.68(\mathrm{~d}, J=0.9 \mathrm{~Hz}, 1 \mathrm{H}), 7.63-7.65(\mathrm{~m}, 1 \mathrm{H}), 7.53-7.57$ $(\mathrm{m}, 1 \mathrm{H}), 7.35-7.39(\mathrm{~m}, 1 \mathrm{H}), 3.20(\mathrm{~s}, 3 \mathrm{H}) .{ }^{13} \mathrm{C} \mathrm{NMR}\left(150 \mathrm{MHz}, \mathrm{CDCl}_{3}\right) \delta: 158.2,157.4,149.5$, 130.1, 127.3, 124.6, 123.5, 122.3, 112.8, 40.6. IR (neat): 3110, 3033, 3013, 2934, 1599, 1539, 1476, 1446, 1287, 1138, 1126, 966, 951, 808, 778, 745, 539, 495. HRMS (ESI-TOF) m/z: calc'd for $[\mathrm{M}+\mathrm{Na}]^{+} 246.0201$, found 246.0390 .

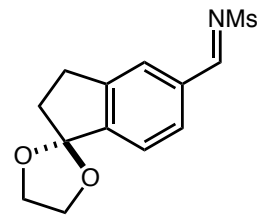

$N$-((2,3-dihydrospiro[indene-1,2'-[1,3]dioxolan]-5-yl)methylene)methanesulfonamide (S21): Aldehyde was synthesized via literature procedure. ${ }^{6}$ To a round bottom flask containing a stir bar, 2,3-dihydrospiro[indene-1,2'-[1,3]dioxolane]-5-carbaldehyde (1.2 eq, $0.67 \mathrm{~g}, 3.32 \mathrm{mmol}$ ) in THF $(3 \mathrm{~mL})$ was added at $0{ }^{\circ} \mathrm{C}$. Next $\mathrm{Ti}\left(\mathrm{O}^{\mathrm{i}} \mathrm{Pr}\right)_{4}(2 \mathrm{eq}, 1.63 \mathrm{~mL}, 5.52 \mathrm{mmol})$ was added. After 5 minutes of stirring, $\mathrm{MsNH}_{2}$ ( $1 \mathrm{eq}, 0.26 \mathrm{~g}, 2.76 \mathrm{mmol}$ ) was added. The reaction mixture was stirred for 10 minutes at $0{ }^{\circ} \mathrm{C}$ and then stirred 24 hours at room temperature. EtOAc and aqueous $\mathrm{NaHCO}_{3}$ were added with vigorously stirring. The reaction was filtered through a pad of $\mathrm{MgSO}_{4} / \mathrm{sand}$ and washed with EtOAc. The combined organic layers were washed with aqueous sodium bicarbonate and brine, then dried over $\mathrm{MgSO}_{4}$, filtered and concentrated under reduced pressure. The crude mixture was purified by column chromatography to provide product $(0.39 \mathrm{~g}, 51 \%)$. $\mathrm{R}_{\mathrm{f}}: 0.25$ (40\% Ethyl acetate/hexanes). ${ }^{1} \mathrm{H}$ NMR $\left(400 \mathrm{MHz}, \mathrm{CDCl}_{3}\right) \delta: 9.03(\mathrm{~s}, 1 \mathrm{H}), 7.82-7.85(\mathrm{~m}, 2 \mathrm{H}), 7.48-7.50$ $(\mathrm{m}, 1 \mathrm{H}), 4.17-4.25(\mathrm{~m}, 2 \mathrm{H}), 4.08-4.15(\mathrm{~m}, 2 \mathrm{H}), 3.13(\mathrm{~s}, 1 \mathrm{H}), 3.01(\mathrm{t}, J=6.9 \mathrm{~Hz}, 1 \mathrm{H}), 2.35(\mathrm{t}$, $J=7.0 \mathrm{~Hz}, 1 \mathrm{H}) .{ }^{13} \mathrm{C} \mathrm{NMR}\left(150 \mathrm{MHz}, \mathrm{CDCl}_{3}\right) \delta: 171.5,149.5,144.8,133.3,130.6,127.8,124.0$, 116.2, 65.4, 40.3, 37.0, 28.2. HRMS (ESI-TOF) $\mathrm{m} / \mathrm{z}$ : calc'd for $[\mathrm{M}+\mathrm{Na}]^{+} 304.0619$, found 304.0867 . 
<smiles>N#Cc1ccc(C=[N+]([O-])[O-])c([N+](=O)[O-])c1</smiles>

N-(4-cyano-2-methylbenzylidene)methanesulfonamide (S22): 4-formyl-3-methylbenzonitrile $(1.5 \mathrm{~g}, 10.3 \mathrm{mmol})$ was subjected to GP1. Recrystallization from ethyl acetate and hexanes provided the product $(900 \mathrm{mg}, 47 \%)$ as a white solid. $\mathrm{R}_{\mathrm{f}}: 0.30\left(15 \%\right.$ Ethyl acetate/hexanes). ${ }^{1} \mathrm{H}$ NMR $\left(400 \mathrm{MHz}, \mathrm{CDCl}_{3}\right) \delta: 9.35(\mathrm{~s}, 1 \mathrm{H}), 8.17(\mathrm{~d}, J=8.6 \mathrm{~Hz}, 1 \mathrm{H}), 7.62(\mathrm{~d}, J=8.6 \mathrm{~Hz}, 1 \mathrm{H}), 7.61$ $(\mathrm{s}, 1 \mathrm{H}), 3.18(\mathrm{~s}, 3 \mathrm{H}), 2.67(\mathrm{~s}, 3 \mathrm{H}) .{ }^{13} \mathrm{C} \mathrm{NMR}\left(100 \mathrm{MHz}, \mathrm{CDCl}_{3}\right) \delta: 168.3,142.9,135.2,134.0$, 130.6, 130.1, 117.8, 117.7, 40.4, 19.5. HRMS (ESI-TOF) $\mathrm{m} / \mathrm{z}$ : calc'd for $[\mathrm{M}+\mathrm{Na}]^{+} 245.0321$, found 245.0362 .<smiles>O=Cc1ccccc1I</smiles>

$N$-(2-iodobenzylidene)methanesulfonamide (S23): 4-iodobenzaldehyde (1.4 eq, $0.67 \mathrm{~g}, 2.88$ mmol) was subjected to GP1. Recrystallization from ethyl acetate and hexanes provided the product (0.40 g, 65\%). Rf: 0.39 (30\% Ethyl acetate/hexanes). ${ }^{1} \mathrm{H}$ NMR (400 MHz, $\left.\mathrm{CDCl}_{3}\right) \delta: 9.29$ (s, 1H), $8.17(\mathrm{dd}, J=1.8,7.8 \mathrm{~Hz}, 1 \mathrm{H}), 7.99$ (ddd, $J=0.3,1.2,8.0 \mathrm{~Hz}, 1 \mathrm{H}), 7.47$ (t, $J=7.5 \mathrm{~Hz}$, 1H), $7.30(\mathrm{~m}, 1 \mathrm{H}), 3.16(\mathrm{~s}, 3 \mathrm{H}) .{ }^{13} \mathrm{C} \mathrm{NMR}\left(150 \mathrm{MHz}, \mathrm{CDCl}_{3}\right) \delta: 175.4,140.8,136.1,133.5,130.8$, 128.9, 104.4, 77.3, 40.4. IR (neat): 3005, 2926, 1597, 1302, 1138, 971, 805, 757, 539, 508, 441. HRMS (ESI-TOF) m/z: calc'd for $[\mathrm{M}+\mathrm{H}]^{+}$309.9399, found 309.9393.<smiles>CN(C)c1cccc(I)c1</smiles>

$N$-(3-iodobenzylidene)methanesulfonamide (S24): 3-iodobenzaldehyde (1.4 eq, $0.65 \mathrm{~g}, 2.8$ mmol) was subjected to GP1. Recrystallization from ethyl acetate and hexanes provided the product (0.33 g, 54\%). Rf: 0.50 (40\% Ethyl acetate/hexanes). ${ }^{1} \mathrm{H}$ NMR (400 MHz, $\left.\mathrm{CDCl}_{3}\right) \delta: 8.94$ (s, 1H), 8.34-8.35 (m, 1H), 7.97-7.99 (m, 1H), 7.88-7.90 (m, 1H), 7.28 (t, J=7.9 Hz, 1H), 3.15 (s, $1 \mathrm{H}) .{ }^{13} \mathrm{C} \mathrm{NMR}\left(150 \mathrm{MHz}, \mathrm{CDCl}_{3}\right) \delta: 170.1,143.8,139.3,134.0,130.9,130.9,94.8,40.4$. IR (neat): 3003, 2923, 2158, 1600, 1548, 1423, 1299, 1275, 1138, 968, 806, 750, 676, 515, 496. HRMS (ESITOF) $\mathrm{m} / \mathrm{z}$ : calc'd for $[\mathrm{M}+\mathrm{Na}]^{+}$331.9218, found 331.9501 . 
<smiles>CN(C)c1ccc(I)cc1</smiles>

$\mathrm{N}$-(4-iodobenzylidene)methanesulfonamide (S25): 4-iodobenzaldehyde (1.4 eq, $0.52 \mathrm{~g}, 2.24$ mmol) was subjected to GP1. Recrystallization from ethyl acetate and hexanes provided the product (0.28 g, 56\%). Rf: 0.41 (30\% Ethyl acetate/hexanes). ${ }^{1} \mathrm{H}$ NMR (400 MHz, $\left.\mathrm{CDCl}_{3}\right) \delta: 8.97$ $(\mathrm{s}, 1 \mathrm{H}), 7.91(\mathrm{~d}, J=8.5 \mathrm{~Hz}, 2 \mathrm{H}), 7.65(\mathrm{~d}, J=8.4 \mathrm{~Hz}, 2 \mathrm{H}), 3.14(\mathrm{~s}, 3 \mathrm{H}) .{ }^{13} \mathrm{C}$ NMR $(150 \mathrm{MHz}$, $\mathrm{CDCl}_{3}$ ) $\delta: 170.8,138.8,132.3,131.5,103.7,40.4$. IR (neat): 3024, 1602, 1582, 1294, 1141, 972, 860, 806, 520, 481. HRMS (ESI-TOF) m/z: calc'd for $[\mathrm{M}+\mathrm{H}]^{+} 309.9399$, found 309.9392.<smiles>O=C(c1ccccc1)c1cccc(C=Nc2ccccc2)c1</smiles>

$N$-(3-benzoylbenzylidene)methanesulfonamide (S26): 3-benzoylbenzaldehyde (1.95 g, 9.28 mmol) was subjected to GP1. Recrystallization from ethyl acetate and hexanes provided the product (750 mg, 35\%) as a white solid. $\mathrm{R}_{\mathrm{f}}: 0.13$ (15\% Ethyl acetate/hexanes). ${ }^{1} \mathrm{H}$ NMR (400 MHz, $\left.\mathrm{CDCl}_{3}\right) \delta: 9.10(\mathrm{~s}, 1 \mathrm{H}), 8.34-8.35(\mathrm{~m}, 1 \mathrm{H}), 8.18-8.21(\mathrm{~m}, 1 \mathrm{H}), 8.08-8.11(\mathrm{~m}, 1 \mathrm{H}), 7.80-7.82$ $(\mathrm{m}, 2 \mathrm{H}), 7.63-7.71(\mathrm{~m}, 2 \mathrm{H}), 7.51-7.55(\mathrm{~m}, 2 \mathrm{H}), 3.15(\mathrm{~s}, 3 \mathrm{H}) .{ }^{13} \mathrm{C}$ NMR $\left(100 \mathrm{MHz}, \mathrm{CDCl}_{3}\right) \delta$ : $195.3,170.8,139.0,136.8,136.1,134.4,133.3,132.6,132.4,130.2,129.6,128.8,40.4$. HRMS (ESI-TOF) $\mathrm{m} / \mathrm{z}$ : calc'd for $[\mathrm{M}+\mathrm{Na}]^{+} 310.0514$, found 310.0525 .<smiles>[N-]=[N+]=CCCC[Mg]</smiles>

4-methyl- $N$-pentylidenebenzenesulfonamide (S27): Pentanal $(0.53 \mathrm{~mL}, 5 \mathrm{mmol}), \quad p$ toluenesulfonamide $(0.85 \mathrm{~g}, 5 \mathrm{mmol})$, and sodium $p$ - toluenesulfinate $(0.89 \mathrm{~g}, 5 \mathrm{mmol})$ was stirred at room temperature in formic acid $(9 \mathrm{~mL})$ and water $(9 \mathrm{~mL})$ for $16 \mathrm{hrs}$. Precipitate was filtered and washed with water then pentanes. The precipitate was dissolved in dichloromethane $(17 \mathrm{~mL})$ and extracted with a solution of aqueous saturated sodium bicarbonate $(17 \mathrm{~mL})$ for $45 \mathrm{sec}$. The organic layer was extracted and dried over $\mathrm{Na}_{2} \mathrm{SO}_{4}$. Solvent was removed under reduced pressure to yield $76 \% \mathbf{S 2 7}$ as a pale yellow oil. ${ }^{1} \mathrm{H}$ NMR (400 $\left.\mathrm{MHz}, \mathrm{CDCl}_{3}\right): \delta=8.60(\mathrm{t}, J=4.6 \mathrm{~Hz}, 1 \mathrm{H})$, $7.81(\mathrm{~d}, J=8.3 \mathrm{~Hz}, 2 \mathrm{H}), 7.34(\mathrm{~d}, J=8.1 \mathrm{~Hz}, 2 \mathrm{H}), 2.51(\mathrm{~m}, 2 \mathrm{H}), 2.44(\mathrm{~s}, 3 \mathrm{H}), 1.60(\mathrm{p}, J=7.5 \mathrm{~Hz}$, $2 \mathrm{H}), 1.41-1.30(\mathrm{~m}, 2 \mathrm{H}), 0.90(\mathrm{t}, J=7.3,3 \mathrm{H}) .{ }^{13} \mathrm{C} \mathrm{NMR}(150 \mathrm{MHz}, \mathrm{CDCl} 3): \delta=178.6,144.8$, $134.9,129.9,128.3,35.8,26.8,22.4,21.8,13.8$. Spectroscopic data is consistent with reported literature data. ${ }^{15}$ 


\section{Synthesis and characterization of aldehydes}<smiles>CC(=O)CCCCC=O</smiles>

methyl 5-oxopentanoate (S30): methyl 5-hydroxypentanoate ( $9.70 \mathrm{mmol}, 1.28 \mathrm{~g})$ was subjected to GP2 then purified by column chromatography. The crude reaction was purified by column chromatography (25\% Et $2 \mathrm{O} /$ Pentanes) to obtain aldehyde $\mathbf{S 3 0}$ (1.23 g, quantitative). ${ }^{1} \mathrm{H}$ NMR (400 $\left.\mathrm{MHz} \mathrm{CDCl}_{3}\right) \delta: 9.77(\mathrm{t}, J=1.3 \mathrm{~Hz}, 1 \mathrm{H}), 3.67(\mathrm{~s}, 3 \mathrm{H}), 2.52(\mathrm{td}, J=7.2,1.3 \mathrm{~Hz}, 2 \mathrm{H}), 2.37(\mathrm{t}, J=$ $7.3 \mathrm{~Hz}, 2 \mathrm{H}), 1.95$ (quint, 3H). ${ }^{13} \mathrm{C} \mathrm{NMR}\left(100 \mathrm{MHz}, \mathrm{CDCl}_{3}\right) \delta: 201.5,173.3,51.6,42.9,32.9,17.3$. Spectral data matched those previously reported. ${ }^{7}$

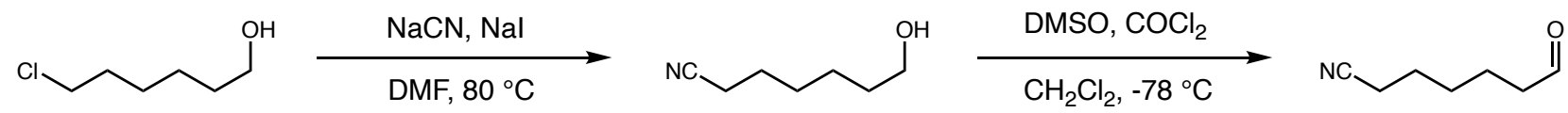<smiles>N#CCCCCCCO</smiles>

7-hydroxyheptanenitrile (S31'): To 6-chlorohexanol (2.67 mL, $20 \mathrm{mmol})$ in DMF (20 mL), NaI (300 mg, $2 \mathrm{mmol})$ and $\mathrm{NaCN}(1.47 \mathrm{~g}, 30 \mathrm{mmol})$ were added. The mixture was then stirred at $80{ }^{\circ} \mathrm{C}$ for 48 hours. The reaction was cooled to room temperature and then extracted with ethyl acetate and washed with $\mathrm{H}_{2} \mathrm{O}$, then brine. The combined organic layers were dried with sodium sulfate, filtered, and purified by column chromatography $\left(2: 1 \mathrm{Et}_{2} \mathrm{O} / \mathrm{PET}\right.$ Ether) to provide the alcohol product (1.72 g, 68\% yield) as a colorless oil. ${ }^{1} \mathrm{H} \mathrm{NMR}\left(400 \mathrm{MHz}, \mathrm{CDCl}_{3}\right) \delta: 3.62(\mathrm{t}, J=6.4 \mathrm{~Hz}$, 2H), $2.33(\mathrm{t}, J=7.1 \mathrm{~Hz}, 2 \mathrm{H}), 1.60-1.70(\mathrm{~m}, 2 \mathrm{H}), 1.53-1.63(\mathrm{~m}, 2 \mathrm{H}), 1.37-1.51(\mathrm{~m}, 2 \mathrm{H}) .{ }^{13} \mathrm{C}$ NMR $\left(100 \mathrm{MHz}, \mathrm{CDCl}_{3}\right) \delta: 119.9,62.6,32.4,28.5,25.4,25.1,17.2$. Spectral data matched those previously reported. ${ }^{8}$<smiles>N#CCCCCCC=O</smiles>

7-oxoheptanenitrile (S31): 7-hydroxyheptanenitrile (1.02 g, $8 \mathrm{mmol})$ was subjected to GP2 then purification by column chromatography $\left(60 \% \mathrm{Et}_{2} \mathrm{O} /\right.$ pentane) provided aldehyde $\mathbf{S 3 1}(915 \mathrm{mg}$, $90 \%$ yield) as a colorless oil. ${ }^{1} \mathrm{H}$ NMR $\left(400 \mathrm{MHz}, \mathrm{CDCl}_{3}\right) \delta: 9.74(\mathrm{~d}, J=1.5 \mathrm{~Hz}, 1 \mathrm{H}), 2.44(\mathrm{dt}, J$ $=1.5,7.2 \mathrm{~Hz}, 1 \mathrm{H}), 2.33(\mathrm{t}, J=7.1 \mathrm{~Hz}, 2 \mathrm{H}), 1.60-1.67(\mathrm{~m}, 4 \mathrm{H}), 1.43-1.49(\mathrm{~m}, 2 \mathrm{H}) .{ }^{13} \mathrm{C} \mathrm{NMR}$ $\left(100 \mathrm{MHz}_{2} \mathrm{CDCl}_{3}\right) \delta: 201.9,116.6,43.5,28.1,25.2,21.2$, 17.0. Spectral data matched those previously reported. ${ }^{8}$ 


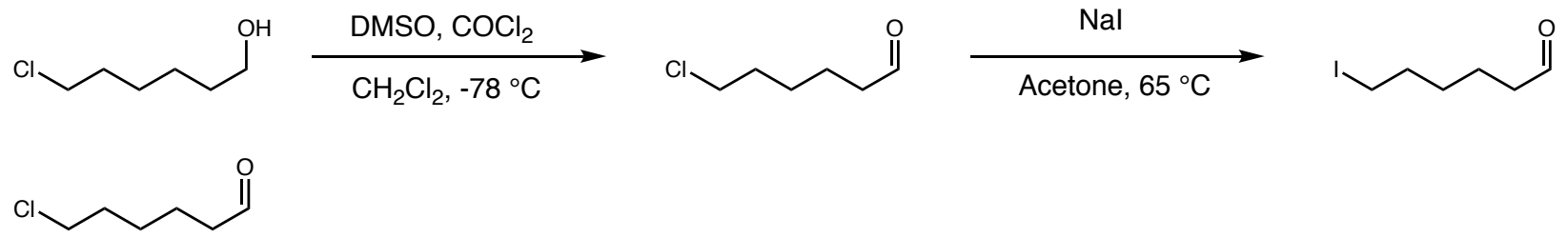

6-chlorohexanal (S32): 6-chlorohexanol $(2.60 \mathrm{~mL}, 19.5 \mathrm{mmol})$ was subjected to GP2, then purification by column chromatography (10:1 to 4:1 pentane/ $\mathrm{Et}_{2} \mathrm{O}$ ) provided aldehyde $\mathbf{S 3 2}$ ( 2.17 $\mathrm{g}, 83 \%$ yield) as slightly yellow oil. ${ }^{1} \mathrm{H}$ NMR $\left(400 \mathrm{MHz}, \mathrm{CDCl}_{3}\right) \delta: 9.72(\mathrm{t}, J=1.6 \mathrm{~Hz}, 1 \mathrm{H}), 3.49$ (t, $J=6.6 \mathrm{~Hz}, 2 \mathrm{H}), 2.42$ (dt, $J=1.6,7.3 \mathrm{~Hz}, 2 \mathrm{H}), 1.72-1.78(\mathrm{~m}, 2 \mathrm{H}), 1.58-1.64(\mathrm{~m}, 2 \mathrm{H}), 1.42$ $-1.46(\mathrm{~m}, 2 \mathrm{H}) .{ }^{13} \mathrm{C}$ NMR $\left(100 \mathrm{MHz}, \mathrm{CDCl}_{3}\right) \delta$ : 202.3, 44.7, 43.7, 32.3, 26.4, 21.3. Spectral data matched those previously reported. ${ }^{9}$<smiles>O=CCCCCCI</smiles>

6-iodohexanal (S33): To 6-chlorohexanal (1.0 g, $7.4 \mathrm{mmol})$ in dry acetone $(40 \mathrm{~mL})$, was added $\mathrm{NaI}(37 \mathrm{mmol}, 5.56 \mathrm{~g})$. The mixture was refluxed for 10 hours. After cooling, the reaction was extracted with $\mathrm{Et}_{2} \mathrm{O}$ then washed with aq $\mathrm{Na}_{2} \mathrm{~S}_{2} \mathrm{O}_{3}$ and brine. The organic layer was dried over sodium sulfate, concentrated, then purification by column chromatography $\left(5 \% \mathrm{Et}_{2} \mathrm{O} /\right.$ pentane) provided aldehyde $\mathbf{S 3 3}\left(1.25 \mathrm{~g}, 75 \%\right.$ yield). ${ }^{1} \mathrm{H}$ NMR $\left(400 \mathrm{MHz}, \mathrm{CDCl}_{3}\right) \delta$ : 9.76 (t, $J=1.6 \mathrm{~Hz}$, $1 \mathrm{H}), 3.18(\mathrm{t}, J=6.9 \mathrm{~Hz}, 2 \mathrm{H}), 2.45(\mathrm{dt}, J=1.6,7.3 \mathrm{~Hz}, 2 \mathrm{H}), 1.79-1.90(\mathrm{~m}, 2 \mathrm{H}), 1.63-1.71(\mathrm{~m}$, $2 \mathrm{H}), 1.42-1.52(\mathrm{~m}, 2 \mathrm{H}) .{ }^{13} \mathrm{C} \mathrm{NMR}\left(100 \mathrm{MHz}, \mathrm{CDCl}_{3}\right) \delta: 202.3,43.8,33.3,30.1,21.1,6.6$. Spectral data matched those previously reported. ${ }^{9}$<smiles>O=CCCO</smiles>

3-((tert-butyldiphenylsilyl)oxy)propanal (S34): 3-((tert-butyldiphenylsilyl)oxy)propan-1-ol (10 $\mathrm{mmol}, 3.15 \mathrm{~g}$ ) was subjected to GP2 then purification by column chromatography (5\% ethyl acetate/hexanes) provided aldehyde $\mathbf{S 3 4}$ as a white solid ( $2.17 \mathrm{~g}, 69 \%$ yield). ${ }^{1} \mathrm{H}$ NMR (400 MHz, $\left.\mathrm{CDCl}_{3}\right) \delta: 9.83(\mathrm{t}, J=2.2 \mathrm{~Hz}, 1 \mathrm{H}), 7.67(\mathrm{~m}, 4 \mathrm{H}), 7.43(\mathrm{~m}, 2 \mathrm{H}), 4.03(\mathrm{t}, J=6.0 \mathrm{~Hz}, 2 \mathrm{H}), 2.61(\mathrm{dt}$, $J=2.2,6.0 \mathrm{~Hz}, 2 \mathrm{H}), 1.05(\mathrm{~s}, 9 \mathrm{H}) .{ }^{13} \mathrm{C}$ NMR $\left(100 \mathrm{MHz}, \mathrm{CDCl}_{3}\right) \delta: 202.0,135.7,133.4,129.9$, $127.9,58.4,46.5,26.9,19.3$. Spectral data matched those previously reported. ${ }^{9}$ 
<smiles>O=CCCN1C(=O)c2ccccc2C1=O</smiles>

3-(1,3-dioxoisoindolin-2-yl)propanal (S35): 2-(3-hydroxypropyl)isoindoline-1,3-dione (5 mmol, $1.02 \mathrm{~g})$ was subjected to GP2 then purification by column chromatography $(50 \%$ ethyl acetate/hexanes) provided aldehyde $\mathbf{S 3 5}$ as a white solid (915 mg, 90\% yield). ${ }^{1} \mathrm{H}$ NMR (400 MHz, $\left.\mathrm{CDCl}_{3}\right) \delta: 9.81(\mathrm{~d}, J=1.4 \mathrm{~Hz}, 1 \mathrm{H}), 7.84(\mathrm{dd}, J=3.0,5.4 \mathrm{~Hz}, 2 \mathrm{H}), 7.72(\mathrm{dd}, J=3.1,5.5 \mathrm{~Hz}, 2 \mathrm{H})$, $4.03(\mathrm{t}, J=7.0 \mathrm{~Hz}, 2 \mathrm{H}), 2.87(\mathrm{dt}, J=1.4,7.0 \mathrm{~Hz}, 2 \mathrm{H}) .{ }^{13} \mathrm{C} \mathrm{NMR}\left(100 \mathrm{MHz}, \mathrm{CDCl}_{3}\right) \delta: 199.4$, $168.0,134.1,132.6,123.4,42.4,31.7$. Spectral data matched those previously reported. ${ }^{9}$<smiles>O=CCCCCCNC(=O)C(F)(F)F</smiles>

2,2,2-trifluoro- $\boldsymbol{N}$-(6-oxohexyl)acetamide (S36): 2,2,2-trifluoro- $N$-(6-hydroxyhexyl)acetamide $(10 \mathrm{mmol}, 2.13 \mathrm{~g})$ was subjected to GP2 then purification by column chromatography (50\% ethyl acetate/hexanes) provided aldehyde $\mathbf{S 3 6}$ as a white solid (1.65 g, 78\% yield). ${ }^{1} \mathrm{H}$ NMR (400 MHz, $\left.\mathrm{CDCl}_{3}\right) \delta: 9.78(\mathrm{t}, J=1.4 \mathrm{~Hz}, 1 \mathrm{H}), 6.30-7.10$ (brs, $\left.1 \mathrm{H}\right), 3.34-3.39(\mathrm{~m}, 2 \mathrm{H}), 2.46-2.50(\mathrm{~m}, 2 \mathrm{H})$, $1.58-1.69(\mathrm{~m}, 4 \mathrm{H}), 1.34-1.41(\mathrm{~m}, 2 \mathrm{H}) .{ }^{13} \mathrm{C} \mathrm{NMR}\left(100 \mathrm{MHz}, \mathrm{CDCl}_{3}\right) \delta: 202.6,157.5\left(\mathrm{q},{ }^{2} J_{\mathrm{CF}}=\right.$ $36.6 \mathrm{~Hz}), 116.0\left(\mathrm{q},{ }^{1} J_{\mathrm{CF}}=287.7 \mathrm{~Hz}\right), 43.7,39.7,28.7,26.2,21.5$. Spectral data matched those previously reported. ${ }^{10}$

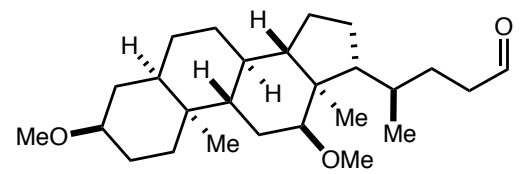

(R)-4-((3R,5R,8R,9S,10S,12S,13R,14S,17R)-3,12-dimethoxy-10,13-dimethylhexadecahydro$1 H$-cyclopenta[a]phenanthren-17-yl)pentanal (S37): The corresponding alcohol (891 mg, 2.2 mmol), synthesized using Gao et al procedure ${ }^{11}$, was subjected to GP2, then purification by column chromatography (15-20\% ethyl acetate/hexanes) provided aldehyde $\mathbf{S 3 7}$ (668 $\mathrm{mg}, 75 \%$ yield) as a white solid. ${ }^{1} \mathrm{H}$ NMR $\left(400 \mathrm{MHz}, \mathrm{CDCl}_{3}\right) \delta: 9.76(\mathrm{t}, J=2.0 \mathrm{~Hz}, 1 \mathrm{H}), 3.37(\mathrm{t}, J=2.4 \mathrm{~Hz}, 1 \mathrm{H}), 3.34$ $(\mathrm{s}, 3 \mathrm{H}), 3.25(\mathrm{~s}, 3 \mathrm{H}), 3.12-3.16(\mathrm{~m}, 1 \mathrm{H}), 2.43-2.48(\mathrm{~m}, 1 \mathrm{H}), 2.33-2.37(\mathrm{~m}, 1 \mathrm{H}), 1.67-1.80$ $(\mathrm{m}, 9 \mathrm{H}), 1.53-1.58(\mathrm{~m}, 3 \mathrm{H}), 1.33-1.41(\mathrm{~m}, 5 \mathrm{H}), 1.09-1.32(\mathrm{~m}, 5 \mathrm{H}), 1.00-1.04(\mathrm{~m}, 1 \mathrm{H}), 0.92$ $-0.98(\mathrm{~m}, 1 \mathrm{H}), 0.91(\mathrm{~s}, 3 \mathrm{H}), 0.90(\mathrm{~d}, J=6.5 \mathrm{~Hz}, 3 \mathrm{H}), 0.66(\mathrm{~s}, 3 \mathrm{H}) .{ }^{13} \mathrm{C} \mathrm{NMR}\left(100 \mathrm{MHz}, \mathrm{CDCl}_{3}\right)$ $\delta: 203.5,82.4,80.6,55.8,55.7,49.0,46.6,46.5,42.2,41.0,36.2,35.5,35.2,34.6,33.7,32.8,28.1$, $27.6,27.5,27.0,26.2,23.8,23.4,22.1,17.6,12.9$. Spectral data matched those previously reported. ${ }^{11}$ 
VII. Synthesis and characterization of aza-pinacol products - imines<smiles>O=C(O)C(c1ccccc1)[C@H](Br)c1ccccc1</smiles>

1-(methylsulfonamido)-1-phenylhexan-2-yl acetate: 1-(methylsulfonamido)-1-phenylhexan2-yl acetate (3c): Imine 2c $(36.6 \mathrm{mg}, 0.2 \mathrm{mmol})$ was subjected to GP3 and upon column chromatography (20\% ethyl acetate/hexanes) provided $90 \%$ 3c (3:1 d.r.) as colorless oil. Rf: 0.43 (30\% ethyl acetate/hexanes). IR (film) $\mathrm{cm}^{-1}: 3273,3090,2957,2930,2861,1319,1231$. HRMS (ESI-TOF) $\mathrm{m} / \mathrm{z}$ : calc'd for $[\mathrm{M}+\mathrm{Na}]^{+} 336.1245$, found 336.1269 .

Major diastereomer: ${ }^{1} \mathrm{H}$ NMR $\left(400 \mathrm{MHz} \mathrm{CDCl}_{3}\right) \delta: 7.32-7.39(\mathrm{~m}, 5 \mathrm{H}), 5.40(\mathrm{~d}, \mathrm{~J}=8.1 \mathrm{~Hz}, 1$ H), $5.11-5.14(\mathrm{~m}, 1 \mathrm{H}), 4.69(\mathrm{dd}, J=4.3,8.2 \mathrm{~Hz}, 1 \mathrm{H}), 2.62(\mathrm{~s}, 3 \mathrm{H}), 2.04(\mathrm{~s}, 3 \mathrm{H}), 1.46-1.48(\mathrm{~m}$, $2 \mathrm{H}), 1.22-1.29(\mathrm{~m}, 4 \mathrm{H}), 0.84(\mathrm{t}, J=6.8 \mathrm{~Hz}, 3 \mathrm{H}) .{ }^{13} \mathrm{C} \mathrm{NMR}\left(150 \mathrm{MHz}, \mathrm{CDCl}_{3}\right) \delta: 171.1,137.2$, $128.9,128.5,127.8,76.2,60.2,42.0,30.0,27.6,22.4,21.1,13.9$.

Minor diastereomer: ${ }^{1} \mathrm{H} \mathrm{NMR}\left(400 \mathrm{MHz}, \mathrm{CDCl}_{3}\right) \delta$ : 7.28-7.38 (m, 5H), $5.72(\mathrm{~d}, J=9.1 \mathrm{~Hz}, 1 \mathrm{H})$, 5.04-5.09 (m, 1H), $4.53(\mathrm{dd}, J=6.0,9.0 \mathrm{~Hz}, 1 \mathrm{H}), 2.55(\mathrm{~s}, 3 \mathrm{H}), 2.05(\mathrm{~s}, 3 \mathrm{H}), 1.44-1.49(\mathrm{~m}, 2 \mathrm{H})$, 1.18-1.30 (m, 4H), 0.80-0.83 (m, 3H). $\left.{ }^{13} \mathrm{C} \mathrm{NMR} \mathrm{(150} \mathrm{MHz,} \mathrm{CDCl}_{3}\right)$ 8: 171.1, 138.7, 129.2, 128.6, 127.0, 76.0, 60.8, 42.1, 31.2, 27.4, 22.4, 21.1, 14.1.<smiles>CC(C)[C@@H](OC(=O)[O-])C(N)c1ccc(C(F)(F)F)cc1</smiles>

1-(methylsulfonamido)-1-(4-(trifluoromethyl)phenyl)hexan-2-yl acetate (5): Imine S5 (50.2 $\mathrm{mg}, 0.2 \mathrm{mmol})$ was subjected to GP3 and upon column chromatography (20\% ethyl acetate/hexanes) provided $92 \% 5$ (2:1 d.r.) as yellow oil. $\mathrm{R}_{\mathrm{f}}$ : 0.53 (30\% ethyl acetate/hexanes). IR (film): 3286, 2933, 2859, 1739, 1611, 1444, 1323, 1231, 1155, 1122, 1067, 1017, 734, 703, 518. HRMS (ESI-TOF) m/z: calc'd for [M+Na] 404.1119 , found 404.1147.

Major diastereomer: ${ }^{1} \mathrm{H} \mathrm{NMR}\left(150 \mathrm{MHz}, \mathrm{CDCl}_{3}\right) \delta: 7.65-7.66(\mathrm{~m}, 2 \mathrm{H}), 7.42-7.46(\mathrm{~m}, 2 \mathrm{H})$, $5.26(\mathrm{~s}, 1 \mathrm{H}), 5.11-5.14(\mathrm{~m}, 1 \mathrm{H}), 4.80(\mathrm{dd}, J=4.0,7.4 \mathrm{~Hz}, 1 \mathrm{H}), 2.70(\mathrm{~s}, 1 \mathrm{H}), 2.06(\mathrm{~s}, 1 \mathrm{H}), 1.42-$ $1.46(\mathrm{~m}, 2 \mathrm{H}), 1.21-1.31(\mathrm{~m}, 4 \mathrm{H}), 0.83-0.87(\mathrm{~m}, 3 \mathrm{H}) .{ }^{13} \mathrm{C} \mathrm{NMR}\left(150 \mathrm{MHz}, \mathrm{CDCl}_{3}\right) \delta: 171.1$, $141.4,130.6\left(\mathrm{q},{ }^{2} J_{\mathrm{CF}}=32.6 \mathrm{~Hz}\right), 128.2,125.7\left(\mathrm{q},{ }^{3} J_{\mathrm{CF}}=3.7 \mathrm{~Hz}\right), 123.9\left(\mathrm{q},{ }^{1} J_{\mathrm{CF}}=272.2 \mathrm{~Hz}\right), 75.9$, 59.7, 42.2, 34.2, 30.1, 27.6, 22.4, 21.1, 13.8. ${ }^{19} \mathrm{~F} \mathrm{NMR}\left(376 \mathrm{MHz}, \mathrm{CDCl}_{3}\right) \delta$ : -62.7.

Minor diastereomer: ${ }^{1} \mathrm{H} \mathrm{NMR}\left(150 \mathrm{MHz}, \mathrm{CDCl}_{3}\right) \delta$ : $7.65-7.66(\mathrm{~m}, 2 \mathrm{H}), 7.42-7.46(\mathrm{~m}, 2 \mathrm{H})$, $5.23(\mathrm{~d}, J=7.3 \mathrm{~Hz}, 1 \mathrm{H}), 5.06-5.09(\mathrm{~m}, 1 \mathrm{H}), 4.67(\mathrm{dd}, J=5.6,8.4 \mathrm{~Hz}, 1 \mathrm{H}), 2.67(\mathrm{~s}, 1 \mathrm{H}), 2.05(\mathrm{~s}$, $1 \mathrm{H}), 1.42-1.46(\mathrm{~m}, 2 \mathrm{H}), 1.21-1.31(\mathrm{~m}, 4 \mathrm{H}), 0.83-0.87(\mathrm{~m}, 3 \mathrm{H}) .{ }^{13} \mathrm{C}$ NMR $\left(150 \mathrm{MHz}, \mathrm{CDCl}_{3}\right)$ $\delta: 170.8,143.0,130.7\left(\mathrm{q},{ }^{2} J_{\mathrm{CF}}=32.6 \mathrm{~Hz}\right), 127.4,126.1\left(\mathrm{q},{ }^{3} J_{\mathrm{CF}}=3.7 \mathrm{~Hz}\right), 123.9\left(\mathrm{q},{ }^{1} J_{\mathrm{CF}}=272.2\right.$ $\mathrm{Hz}), 75.8,60.1,42.3,34.2,31.2,27.3,22.4,21.0,14.1 .{ }^{19} \mathrm{~F} \mathrm{NMR}\left(376 \mathrm{MHz}, \mathrm{CDCl}_{3}\right) \delta$ : -62.7 . 


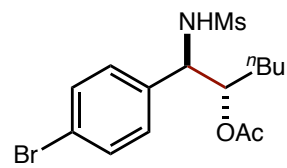

1-(4-bromophenyl)-1-(methylsulfonamido)hexan-2-yl acetate (6): Imine S6 (52.4 mg, 0.2 mmol) was subjected to GP3 and upon column chromatography (20\% ethyl acetate/hexanes) provided 89\% 6 (3:1 d.r.) as colorless oil. $\mathrm{R}_{\mathrm{f}}$ : 0.42 (30\% ethyl acetate/hexanes). IR (film): 3280, 2956, 2931, 2861, 2361, 1739, 1489, 1439, 1373, 1318, 1230, 1155, 1121, 1073, 1025, 1010, 980, 760, 519. HRMS (ESI-TOF) m/z: calc'd for $[\mathrm{M}+\mathrm{Na}]^{+} 414.0351$, found 414.0348.

Major diastereomer: ${ }^{1} \mathrm{H} \mathrm{NMR}\left(150 \mathrm{MHz}, \mathrm{CDCl}_{3}\right) \delta: 7.50-7.52(\mathrm{~m}, 2 \mathrm{H}), 7.17-7.21(\mathrm{~m}, 2 \mathrm{H})$, $5.26(\mathrm{~s}, 1 \mathrm{H}), 5.07-5.10(\mathrm{~m}, 1 \mathrm{H}), 4.67(\mathrm{dd}, J=4.2,7.2 \mathrm{~Hz}, 1 \mathrm{H}), 2.67(\mathrm{~s}, 3 \mathrm{H}), 2.05(\mathrm{~s}, 3 \mathrm{H}), 1.41-$ $1.45(\mathrm{~m}, 2 \mathrm{H}), 1.20-1.29(\mathrm{~m}, 4 \mathrm{H}), 0.83-0.86(\mathrm{~m}, 3 \mathrm{H}) .{ }^{13} \mathrm{C} \mathrm{NMR}\left(150 \mathrm{MHz}, \mathrm{CDCl}_{3}\right) \delta: 171.1$, $136.3,132.0,129.5,122.5,75.9,59.6,42.1,30.1,27.5,22.3,21.0,13.9$.

Minor diastereomer: ${ }^{1} \mathrm{H}$ NMR $\left(150 \mathrm{MHz}^{\mathrm{CDCl}} 3\right) \delta: 7.50-7.52(\mathrm{~m}, 2 \mathrm{H}), 7.17-7.21(\mathrm{~m}, 2 \mathrm{H})$, $5.20(\mathrm{~d}, J=8.2 \mathrm{~Hz}, 1 \mathrm{H}), 5.02-5.05(\mathrm{~m}, 1 \mathrm{H}), 4.54(\mathrm{dd}, J=5.9,8.3 \mathrm{~Hz}, 1 \mathrm{H}), 2.63(\mathrm{~s}, 3 \mathrm{H}), 2.07(\mathrm{~s}$, $3 \mathrm{H}), 1.41-1.45(\mathrm{~m}, 2 \mathrm{H}), 1.20-1.29(\mathrm{~m}, 4 \mathrm{H}), 0.83-0.86(\mathrm{~m}, 3 \mathrm{H}) .{ }^{13} \mathrm{C} \mathrm{NMR}\left(150 \mathrm{MHz}, \mathrm{CDCl}_{3}\right)$ $\delta: 171.0,137.9,132.4,128.7,122.6,75.8,60.1,42.3,31.1,27.3,22.3,21.0,13.9$.

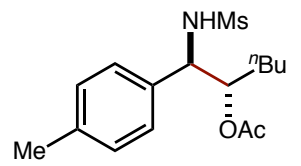

1-(methylsulfonamido)-1-(p-tolyl)hexan-2-yl acetate (7): Imine S7 (39.4 mg, $0.2 \mathrm{mmol}$ ) was subjected to GP3 and upon column chromatography (20\% ethyl acetate/hexanes) provided 75\% 7 (3:1 d.r.) as colorless oil. $\mathrm{R}_{\mathrm{f}}$ : 0.39 (30\% ethyl acetate/hexanes). IR (film): 3282, 2956, 2930, 2861, 2360, 1737, 1515, 1439, 1373, 1317, 1230, 1154, 1021, 979, 729, 516. HRMS (ESI-TOF) m/z: calc'd for $[\mathrm{M}+\mathrm{Na}]^{+} 350.1402$, found 350.1429 .

Major diastereomer: ${ }^{1} \mathrm{H} \mathrm{NMR}\left(400 \mathrm{MHz}, \mathrm{CDCl}_{3}\right) \delta: 7.15-7.22(\mathrm{~m}, 4 \mathrm{H}), 5.54(\mathrm{~d}, J=8.6 \mathrm{~Hz}$, $1 \mathrm{H}), 5.09-5.13(\mathrm{~m}, 1 \mathrm{H}), 4.62(\mathrm{dd}, J=4.3,8.3 \mathrm{~Hz}, 1 \mathrm{H}), 2.60(\mathrm{~s}, 3 \mathrm{H}), 2.33(\mathrm{~s}, 3 \mathrm{H}), 2.03(\mathrm{~s}, 3 \mathrm{H})$, $1.42-1.48(\mathrm{~m}, 2 \mathrm{H}), 1.18-1.31(\mathrm{~m}, 4 \mathrm{H}), 0.81-0.84(\mathrm{~m}, 3 \mathrm{H}) .{ }^{13} \mathrm{C}$ NMR $\left(150 \mathrm{MHz}, \mathrm{CDCl}_{3}\right) \delta$ : $171.0,138.2,134.1,129.5,127.7,76.2,59.9,42.0,30.1,27.6,22.4,21.2,21.1,13.9$.

Minor diastereomer: ${ }^{1} \mathrm{H} \mathrm{NMR}\left(400 \mathrm{MHz}, \mathrm{CDCl}_{3}\right) \delta$ : $7.15-7.22(\mathrm{~m}, 4 \mathrm{H}), 5.40(\mathrm{~d}, J=9.0 \mathrm{~Hz}$, $1 \mathrm{H}), 5.03-5.07(\mathrm{~m}, 1 \mathrm{H}), 4.49(\mathrm{dd}, J=6.2,9.0 \mathrm{~Hz}, 1 \mathrm{H}), 2.55(\mathrm{~s}, 3 \mathrm{H}), 2.33(\mathrm{~s}, 3 \mathrm{H}), 2.07(\mathrm{~s}, 3 \mathrm{H})$, $1.42-1.48(\mathrm{~m}, 2 \mathrm{H}), 1.18-1.31(\mathrm{~m}, 4 \mathrm{H}), 0.81-0.84(\mathrm{~m}, 3 \mathrm{H}) .{ }^{13} \mathrm{C} \mathrm{NMR}\left(150 \mathrm{MHz}, \mathrm{CDCl}_{3}\right) \delta$ : $171.1,138.3,135.6,129.8,126.9,76.0,60.6,42.0,31.2,27.4,22.4,21.2,21.1,13.9$. 


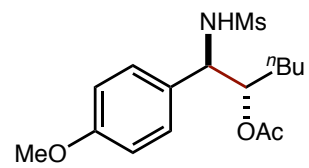

1-(4-methoxyphenyl)-1-(methylsulfonamido)hexan-2-yl acetate (8): Imine S8 (42.6 mg, 0.2 mmol) was subjected to GP3 and upon column chromatography (25\% ethyl acetate/hexanes) provided 54\% 8 (3:1 d.r.) as colorless oil. $\mathrm{R}_{\mathrm{f}}$ : 0.50 (50\% ethyl acetate/hexanes). IR (film): 3278 , 2956, 2931, 2859, 2359, 2340, 1738, 1611, 1513, 1441, 1372, 1318, 1233, 1180, 1154, 1030, 980, 833, 753, 521. HRMS (ESI-TOF) m/z: calc'd for [M+Na] 366.1351 , found 366.1355.

Major diastereomer: ${ }^{1} \mathrm{H}$ NMR $\left(400 \mathrm{MHz}, \mathrm{CDCl}_{3}\right) \delta: 7.19-7.24(\mathrm{~m}, 2 \mathrm{H}), 6.88-6.91(\mathrm{~m}, 2 \mathrm{H})$, $5.25(\mathrm{~d}, J=7.9 \mathrm{~Hz}, 1 \mathrm{H}), 5.08-5.12(\mathrm{~m}, 1 \mathrm{H}), 4.62(\mathrm{dd}, J=4.3,7.9 \mathrm{~Hz}, 1 \mathrm{H}), 3.81(\mathrm{~s}, 3 \mathrm{H}), 2.61(\mathrm{~s}$, $3 \mathrm{H}), 2.04(\mathrm{~s}, 3 \mathrm{H}), 1.42-1.50(\mathrm{~m}, 2 \mathrm{H}), 1.19-1.32(\mathrm{~m}, 4 \mathrm{H}), 0.82-0.85(\mathrm{~m}, 3 \mathrm{H}) .{ }^{13} \mathrm{C}$ NMR $(150$ $\left.\mathrm{MHz} \mathrm{CDCl}_{3}\right) \delta: 171.1,159.6,129.0,128.3,114.2,76.3,59.7,55.4,42.1,30.2,27.6,22.4,21.1$, 14.0 .

Minor diastereomer: ${ }^{1} \mathrm{H} \mathrm{NMR}\left(400 \mathrm{MHz}, \mathrm{CDCl}_{3}\right) \delta: 7.19-7.24(\mathrm{~m}, 2 \mathrm{H}), 6.88-6.91(\mathrm{~m}, 2 \mathrm{H})$, $5.17(\mathrm{~d}, J=8.5 \mathrm{~Hz}, 1 \mathrm{H}), 5.01-5.06(\mathrm{~m}, 1 \mathrm{H}), 4.49(\mathrm{dd}, J=6.5,8.5 \mathrm{~Hz}, 1 \mathrm{H}), 3.80(\mathrm{~s}, 3 \mathrm{H}), 2.55(\mathrm{~s}$, $3 \mathrm{H}), 2.08(\mathrm{~s}, 3 \mathrm{H}), 1.42-1.50(\mathrm{~m}, 2 \mathrm{H}), 1.19-1.32(\mathrm{~m}, 4 \mathrm{H}), 0.82-0.85(\mathrm{~m}, 3 \mathrm{H}) .{ }^{13} \mathrm{C}$ NMR $(150$ $\left.\mathrm{MHz}_{\mathrm{CDCl}}\right)$ ) $: 171.2,159.7,130.6,129.1,114.6,76.0,60.3,55.4,42.1,31.2,27.4,22.4,21.1$, 14.0 .

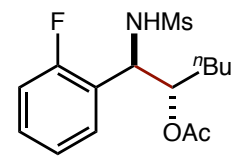

1-(2-fluorophenyl)-1-(methylsulfonamido)hexan-2-yl acetate (9): Imine S9 (40.2 mg, 0.2 mmol) was subjected to GP3 and upon column chromatography (15\% ethyl acetate/hexanes) provided 90\% 9 (1:1 d.r.) as colorless oil. $\mathrm{R}_{\mathrm{f}}$ : 0.34 (30\% ethyl acetate/hexanes). IR (film): 3280, 2957, 2861, 2360, 2341, 1738, 1595, 1570, 1442, 1321, 1275, 1259, 1229, 1154, 979, 764, 750, 520. HRMS (ESI-TOF) m/z: calc'd for $[\mathrm{M}+\mathrm{Na}]^{+} 354.1151$, found 354.1145.

Major diastereomer: ${ }^{1} \mathrm{H} \mathrm{NMR}\left(400 \mathrm{MHz}, \mathrm{CDCl}_{3}\right) \delta: 7.27-7.36(\mathrm{~m}, 2 \mathrm{H}), 7.13-7.17(\mathrm{~m}, 1 \mathrm{H})$, $7.05-7.09(\mathrm{~m}, 1 \mathrm{H}), 5.75(\mathrm{~d}, J=8.9 \mathrm{~Hz}, 1 \mathrm{H}), 5.17-5.22(\mathrm{~m}, 1 \mathrm{H}), 4.86-4.89(\mathrm{~m}, 1 \mathrm{H}), 2.67(\mathrm{~s}$, $3 \mathrm{H}), 1.95(\mathrm{~s}, 3 \mathrm{H}), 1.46-1.65(\mathrm{~m}, 2 \mathrm{H}), 1.24-1.30(\mathrm{~m}, 4 \mathrm{H}), 0.82-0.85(\mathrm{~m}, 3 \mathrm{H}) .{ }^{13} \mathrm{C}$ NMR $(150$ $\left.\mathrm{MHz}, \mathrm{CDCl}_{3}\right) \delta: 170.7,160.5\left(\mathrm{~d},{ }^{1} J_{\mathrm{CF}}=246.6 \mathrm{~Hz}\right), 130.2,129.8\left(\mathrm{~d},{ }^{3} J_{\mathrm{CF}}=4.0 \mathrm{~Hz}\right), 124.6\left(\mathrm{~d},{ }^{2} J_{\mathrm{CF}}\right.$ $=15.0 \mathrm{~Hz}), 124.75\left(\mathrm{~d},{ }^{3} J_{\mathrm{CF}}=5.5 \mathrm{~Hz}\right), 116.0\left(\mathrm{~d},{ }^{2} J_{\mathrm{CF}}=22.5 \mathrm{~Hz}\right), 75.4,55.2,41.6,31.0,27.2,22.4$, 20.9, 13.9. ${ }^{19} \mathrm{~F}$ NMR $\delta:-116.60\left(377 \mathrm{MHz}, \mathrm{CDCl}_{3}\right)$.

Minor diastereomer: ${ }^{1} \mathrm{H}$ NMR $\left(400 \mathrm{MHz} \mathrm{CDCl}_{3}\right) \delta: 7.27-7.36(\mathrm{~m}, 2 \mathrm{H}), 7.13-7.17(\mathrm{~m}, 1 \mathrm{H})$, $7.05-7.09(\mathrm{~m}, 1 \mathrm{H}), 5.64(\mathrm{~d}, J=9.6 \mathrm{~Hz}, 1 \mathrm{H}), 5.12-5.17(\mathrm{~m}, 1 \mathrm{H}), 4.82-4.86(\mathrm{~m}, 1 \mathrm{H}), 2.67(\mathrm{~s}$, $3 \mathrm{H}), 2.03(\mathrm{~s}, 3 \mathrm{H}), 1.46-1.65(\mathrm{~m}, 2 \mathrm{H}), 1.24-1.30(\mathrm{~m}, 4 \mathrm{H}), 0.82-0.85(\mathrm{~m}, 3 \mathrm{H}) .{ }^{13} \mathrm{C}$ NMR $(150$ $\left.\mathrm{MHz}, \mathrm{CDCl}_{3}\right) \delta: 170.7,160.1\left(\mathrm{~d},{ }^{1} J_{\mathrm{CF}}=246.5 \mathrm{~Hz}\right), 130.1,128.5\left(\mathrm{~d},{ }^{3} J_{\mathrm{CF}}=4.0 \mathrm{~Hz}\right), 125.9\left(\mathrm{~d},{ }^{2} J_{\mathrm{CF}}\right.$ $=13.1 \mathrm{~Hz}), 124.5\left(\mathrm{~d},{ }^{3} J_{\mathrm{CF}}=3.4 \mathrm{~Hz}\right), 116.1\left(\mathrm{~d},{ }^{2} J_{\mathrm{CF}}=21.8 \mathrm{~Hz}\right), 75.0,55.4,41.8,30.7,27.4,22.3$, 20.9, 13.9. ${ }^{19} \mathrm{~F}$ NMR $\delta:-117.54\left(377 \mathrm{MHz}, \mathrm{CDCl}_{3}\right)$. 


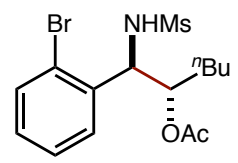

1-(2-bromophenyl)-1-(methylsulfonamido)hexan-2-yl acetate (10): Imine S10 (52.4 mg, 0.2 mmol) was subjected to GP3 and upon column chromatography (15\% ethyl acetate/hexanes) provided $86 \% 10$ (2:1 d.r.) as colorless oil. $\mathrm{R}_{\mathrm{f}}$ : 0.30 (30\% ethyl acetate/hexanes). IR (film): 3282 , 2958, 2861, 2360, 2255, 1737, 1439, 1324, 1275, 1260, 1229, 1155, 1024, 978, 906, 749, 726, 647, 518. HRMS (ESI-TOF) m/z: calc'd for $[\mathrm{M}+\mathrm{Na}]^{+} 414.0351$, found 414.0336.

Major diastereomer: ${ }^{1} \mathrm{H} \mathrm{NMR}\left(400 \mathrm{MHz}, \mathrm{CDCl}_{3}\right) \delta$ : $7.55-7.57(\mathrm{~m}, 1 \mathrm{H}), 7.30-7.45(\mathrm{~m}, 2 \mathrm{H})$, $7.14-7.19(\mathrm{~m}, 1 \mathrm{H}), 5.91-5.95(\mathrm{~m}, 1 \mathrm{H}), 5.09-5.29(\mathrm{~m}, 2 \mathrm{H}), 2.65(\mathrm{~s}, 3 \mathrm{H}), 1.96(\mathrm{~s}, 3 \mathrm{H}), 1.45-$ $1.70(\mathrm{~m}, 2 \mathrm{H}), 1.24-1.29(\mathrm{~m}, 4 \mathrm{H}), 0.81-0.88(\mathrm{~m}, 3 \mathrm{H}) .{ }^{13} \mathrm{C} \mathrm{NMR}\left(150 \mathrm{MHz}, \mathrm{CDCl}_{3}\right) \delta: 170.4$, 137.2, 133.4, 129.9, 128.0, 124.0, 75.0, 59.0, 41.7, 30.6, 27.5, 22.4, 20.9, 14.0.

Minor diastereomer: ${ }^{1} \mathrm{H} \mathrm{NMR}\left(400 \mathrm{MHz}, \mathrm{CDCl}_{3}\right) \delta: 7.55-7.57(\mathrm{~m}, 1 \mathrm{H}), 7.30-7.45(\mathrm{~m}, 2 \mathrm{H})$, $7.14-7.19(\mathrm{~m}, 1 \mathrm{H}), 5.91-5.95(\mathrm{~m}, 1 \mathrm{H}), 5.09-5.29(\mathrm{~m}, 2 \mathrm{H}), 2.66(2,3 \mathrm{H}), 1.99(\mathrm{~s}, 3 \mathrm{H}), 1.45-$ $1.70(\mathrm{~m}, 2 \mathrm{H}), 1.24-1.29(\mathrm{~m}, 4 \mathrm{H}), 0.81-0.88(\mathrm{~m}, 3 \mathrm{H}) .{ }^{13} \mathrm{C} \mathrm{NMR}\left(150 \mathrm{MHz}, \mathrm{CDCl}_{3}\right), \delta: 170.4$, $138.0,133.7,129.7,127.9,123.0,75.0,59.0,42.0,31.2,27.3,22.4,20.9,13.9$.<smiles>CC(=O)OC([18O])[C@H]([NH3+])c1ccccc1[N+](=O)[O-]</smiles>

1-(methylsulfonamido)-1-(o-tolyl)hexan-2-yl acetate (11): Imine S11 (59.2 mg, $0.2 \mathrm{mmol})$ was subjected to GP3 and upon column chromatography (15 - 25\% ethyl acetate/hexanes) provide $81 \% 11$ (7:1 d.r.) as colorless oil. $\mathrm{R}_{\mathrm{f}}: 0.35$ (30\% ethyl acetate/hexanes). HRMS (ESI-TOF) m/z: calc'd for $[\mathrm{M}+\mathrm{Na}]^{+} 350.1402$, found 350.1413 .

Major diastereomer: ${ }^{1} \mathrm{H} \mathrm{NMR}\left(400 \mathrm{MHz}_{\mathrm{CDCl}}\right) \delta: 7.19-7.32(\mathrm{~m}, 4 \mathrm{H}), 5.08-5.13(\mathrm{~m}, 2 \mathrm{H})$, $5.02(\mathrm{dd}, J=5.3,7.8 \mathrm{~Hz}, 1 \mathrm{H}), 2.62(\mathrm{~s}, 3 \mathrm{H}), 2.49(\mathrm{~s}, 3 \mathrm{H}), 2.02(\mathrm{~s}, 3 \mathrm{H}), 1.50-1.69(\mathrm{~m}, 2 \mathrm{H}), 1.19-$ $1.38(\mathrm{~m}, 4 \mathrm{H}), 0.87(\mathrm{t}, J=6.9 \mathrm{~Hz}, 3 \mathrm{H}) .{ }^{13} \mathrm{C} \mathrm{NMR}\left(100 \mathrm{MHz}, \mathrm{CDCl}_{3}\right) \delta: 170.8,136.5,136.0,130.9$, $128.2,127.0,126.6,75.2,56.1,42.0,29.5,27.7,22.5,21.0,19.6,14.0$.

Minor diastereomer: ${ }^{1} \mathrm{H}$ NMR $\left(400 \mathrm{MHz} \mathrm{CDCl}_{3}\right) \delta: 7.19-7.32(\mathrm{~m}, 4 \mathrm{H}), 5.25(\mathrm{~d}, J=8.6 \mathrm{~Hz}, 1$ H) $5.08-5.13(\mathrm{~m}, 1 \mathrm{H}), 4.91(\mathrm{dd}, J=5.8,8.6 \mathrm{~Hz}, 1 \mathrm{H}), 2.54(\mathrm{~s}, 3 \mathrm{H}), 2.44(\mathrm{~s}, 3 \mathrm{H}), 2.09(\mathrm{~s}, 3 \mathrm{H}), 1.50$ - $1.69(\mathrm{~m}, 2 \mathrm{H}), 1.19-1.38(\mathrm{~m}, 4 \mathrm{H}), 0.87(\mathrm{t}, J=6.9 \mathrm{~Hz}, 3 \mathrm{H}) \cdot{ }^{13} \mathrm{C}$ NMR $\left(100 \mathrm{MHz}, \mathrm{CDCl}_{3}\right) \delta$ : $171.1,137.0,135.9,131.3,128.3,126.8,126.2$, 126.6, 75.6, 56.4, 42.1, 31.1, 27.6, 21.1, 19.6, 14.0. 


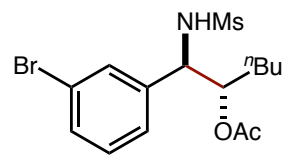

1-(3-bromophenyl)-1-(methylsulfonamido)hexan-2-yl acetate (12): Imine S12 (52.4 mg, 0.2 mmol) was subjected to GP3 and upon column chromatography (15\% ethyl acetate/hexanes) provides $71 \% 12$ (2:1 d.r.) as colorless oil. $\mathrm{R}_{\mathrm{f}}$ : 0.36 (30\% ethyl acetate/hexanes). IR (film): 3279, 2957, 2861, 2359, 1735, 1570, 1439, 1373, 1321, 1275, 1260, 1229, 1154, 978, 907, 749, 520. HRMS (ESI-TOF) m/z: calc'd for $[\mathrm{M}+\mathrm{Na}]^{+} 414.0351$, found 414.0341 .

Major diastereomer: ${ }^{1} \mathrm{H} \mathrm{NMR}\left(400 \mathrm{MHz}, \mathrm{CDCl}_{3}\right) \delta$ : $7.44-7.49(\mathrm{~m}, 2 \mathrm{H}), 7.23-7.28(\mathrm{~m}, 1 \mathrm{H})$, $7.16-7.19(\mathrm{~m}, 1 \mathrm{H}), 5.58(\mathrm{~d}, J=8.0 \mathrm{~Hz}, 1 \mathrm{H}), 5.08-5.12(\mathrm{~m}, 1 \mathrm{H}), 4.66(\mathrm{dd}, J=4.3,7.6 \mathrm{~Hz}, 1 \mathrm{H})$, $2.69(\mathrm{~s}, 3 \mathrm{H}), 2.06(\mathrm{~s}, 3 \mathrm{H}), 1.41-1.46(\mathrm{~m}, 2 \mathrm{H}), 1.19-1.32(\mathrm{~m}, 4 \mathrm{H}), 0.82-0.87(\mathrm{~m}, 3 \mathrm{H}) .{ }^{13} \mathrm{C} \mathrm{NMR}$ $\left(150 \mathrm{MHz}, \mathrm{CDCl}_{3}\right) \delta: 171.1,139.7,131.6,130.9,130.4,126.4,122.9,75.9,59.6,42.2,30.0,27.6$, 22.4, 21.1, 13.9 .

Minor diastereomer: ${ }^{1} \mathrm{H}$ NMR $\left(400 \mathrm{MHz}, \mathrm{CDCl}_{3}\right) \delta: 7.44-7.49(\mathrm{~m}, 2 \mathrm{H}), 7.23-7.28(\mathrm{~m}, 1 \mathrm{H})$, $7.16-7.19(\mathrm{~m}, 1 \mathrm{H}), 5.48(\mathrm{~d}, J=8.9 \mathrm{~Hz}, 1 \mathrm{H}), 5.01-5.06(\mathrm{~m}, 1 \mathrm{H}), 4.53(\mathrm{dd}, J=5.7,8.7 \mathrm{~Hz}, 1 \mathrm{H})$, $2.65(\mathrm{~s}, 3 \mathrm{H}), 2.07(\mathrm{~s}, 3 \mathrm{H}), 1.41-1.46(\mathrm{~m}, 2 \mathrm{H}), 1.19-1.32(\mathrm{~m}, 4 \mathrm{H}), 0.82-0.87(\mathrm{~m}, 3 \mathrm{H}) .{ }^{13} \mathrm{C} \mathrm{NMR}$ $\left(150 \mathrm{MHz}, \mathrm{CDCl}_{3}\right) \delta: 170.9,141.2,131.7,130.7,130.1,125.7,123.2,75.9,60.1,42.3,31.2,27.4$, 22.4, 21.0, 13.9 .<smiles>Cc1cccc(C(C(O)C(=O)O)[N+](=O)[O-])c1</smiles>

1-(methylsulfonamido)-1-(m-tolyl)hexan-2-yl acetate (13): Imine S13 (39.4 mg, $0.2 \mathrm{mmol})$ was subjected to GP3 and upon column chromatography (15\% ethyl acetate/hexanes) provides 79\% 13 (3:1 d.r.) as colorless oil. Rf: 0.39 (30\% ethyl acetate/hexanes). IR (film): 3279, 2930, 2860, 2360, 1736, 1609, 1443, 1372, 1316, 1274, 1260, 1229, 1152, 979, 764, 749, 707, 520. HRMS (ESITOF) $\mathrm{m} / \mathrm{z}$ : calc'd for $[\mathrm{M}+\mathrm{Na}]^{+} 350.1402$, found 350.1401 .

Major diastereomer: ${ }^{1} \mathrm{H} \mathrm{NMR}\left(400 \mathrm{MHz}, \mathrm{CDCl}_{3}\right) \delta: 7.23-7.27(\mathrm{~m}, 1 \mathrm{H}), 7.08-7.13(\mathrm{~m}, 3 \mathrm{H})$, $5.53(\mathrm{~d}, J=8.8 \mathrm{~Hz}, 1 \mathrm{H}), 5.08-5.12(\mathrm{~m}, 1 \mathrm{H}), 4.63(\mathrm{dd}, J=4.3,8.8 \mathrm{~Hz}, 1 \mathrm{H}), 2.62(\mathrm{~s}, 3 \mathrm{H}), 2.35(\mathrm{~s}$, $3 \mathrm{H}), 2.03(\mathrm{~s}, 3 \mathrm{H}), 1.44-1.50(\mathrm{~m}, 2 \mathrm{H}), 1.22-1.29(\mathrm{~m}, 4 \mathrm{H}), 0.81-0.89(\mathrm{~m}, 3 \mathrm{H}) .{ }^{13} \mathrm{C}$ NMR $(150$ $\left.\mathrm{MHz}, \mathrm{CDCl}_{3}\right) \delta: 171.0,138.5,137.1,129.1,128.7,128.5,124.6,76.2,60.1,42.0,29.9,27.6,22.4$, 21.5, 21.1, 13.9

Minor diastereomer: ${ }^{1} \mathrm{H}$ NMR $\left(400 \mathrm{MHz}, \mathrm{CDCl}_{3}\right) \delta: 7.23-7.27(\mathrm{~m}, 1 \mathrm{H}), 7.08-7.13(\mathrm{~m}, 3 \mathrm{H})$, $5.40(\mathrm{~d}, J=9.1 \mathrm{~Hz}, 1 \mathrm{H}), 5.03-5.08(\mathrm{~m}, 1 \mathrm{H}), 4.48(\mathrm{dd}, J=6.2,9.1 \mathrm{~Hz}, 1 \mathrm{H}), 2.55(\mathrm{~s}, 3 \mathrm{H}), 2.35(\mathrm{~s}$, $3 \mathrm{H}), 2.07(\mathrm{~s}, 3 \mathrm{H}), 1.44-1.50(\mathrm{~m}, 2 \mathrm{H}), 1.22-1.29(\mathrm{~m}, 4 \mathrm{H}), 0.81-0.89(\mathrm{~m}, 3 \mathrm{H}) .{ }^{13} \mathrm{C} \mathrm{NMR}(150$ $\left.\mathrm{MHz}_{2} \mathrm{CDCl}_{3}\right) \delta: 171.1,138.9,138.6,129.3,129.0,127.8,124.0,76.0,60.8,42.0,31.2,27.4,22.4$, 21.5, 21.1, 14.1. 


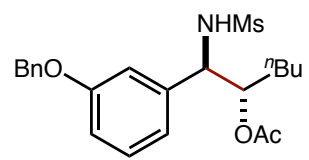

1-(3-(benzyloxy)phenyl)-1-(methylsulfonamido)hexan-2-yl acetate (14): Imine S14 (57.8 mg, $0.2 \mathrm{mmol}$ ) was subjected to GP3 and upon column chromatography (15\% ethyl acetate/hexanes) provides $79 \% 14$ (3:1 d.r.) as colorless oil. $\mathrm{R}_{\mathrm{f}}$ : 0.42 (30\% ethyl acetate/hexanes). IR (film): 3281 , 2930, 2858, 2359, 1738, 1608, 1452, 1372, 1317, 1274, 1259, 1231, 1154, 1025, 981, 764, 749, 698. HRMS (ESI-TOF) m/z: calc'd for $[\mathrm{M}+\mathrm{Na}]^{+} 442.1664$, found 442.1662 .

Major diastereomer: ${ }^{1} \mathrm{H} \mathrm{NMR}\left(400 \mathrm{MHz}, \mathrm{CDCl}_{3}\right) \delta: 7.28-7.44(\mathrm{~m}, 6 \mathrm{H}), 6.88-6.97(\mathrm{~m}, 3 \mathrm{H})$, $5.79(\mathrm{~d}, J=8.5 \mathrm{~Hz}, 1 \mathrm{H}), 5.09-5.14(\mathrm{~m}, 1 \mathrm{H}), 5.08(\mathrm{~s}, 2 \mathrm{H}), 4.64(\mathrm{dd}, J=4.3,8.1 \mathrm{~Hz}, 1 \mathrm{H}), 2.56(\mathrm{~s}$, $3 \mathrm{H}), 2.03(\mathrm{~s}, 3 \mathrm{H}), 1.42-1.47(\mathrm{~m}, 2 \mathrm{H}), 1.20-1.29(\mathrm{~m}, 4 \mathrm{H}), 0.81-0.85(\mathrm{~m}, 3 \mathrm{H}) .{ }^{13} \mathrm{C}$ NMR $(150$ $\left.\mathrm{MHz} \mathrm{CDCl}_{3}\right) \delta: 170.9,158.9,138.8,136.8,129.8,128.7,128.1,127.5,120.3,114.9,114.2,76.1$, 70.0, 59.9, 41.8, 29.9, 27.6, 22.3, 21.1, 13.89

Minor diastereomer: ${ }^{1} \mathrm{H} \mathrm{NMR}\left(400 \mathrm{MHz} \mathrm{CDCl}_{3}\right) \delta: 7.28-7.44(\mathrm{~m}, 6 \mathrm{H}), 6.88-6.97(\mathrm{~m}, 3 \mathrm{H})$, $5.63(\mathrm{~d}, J=9.2 \mathrm{~Hz}, 1 \mathrm{H}), 5.08(\mathrm{~s}, 2 \mathrm{H}), 5.03-5.07(\mathrm{~m}, 1 \mathrm{H}), 4.48(\mathrm{dd}, J=6.3,9.0 \mathrm{~Hz}, 1 \mathrm{H}), 2.52(\mathrm{~s}$, $3 \mathrm{H}), 2.06(\mathrm{~s}, 3 \mathrm{H}), 1.42-1.47(\mathrm{~m}, 2 \mathrm{H}), 1.20-1.29(\mathrm{~m}, 4 \mathrm{H}), 0.81-0.85(\mathrm{~m}, 3 \mathrm{H}) .{ }^{13} \mathrm{C}$ NMR $(150$ $\left.\mathrm{MHz}, \mathrm{CDCl}_{3}\right) \delta: 171.1,159.2,140.2,136.7,130.2,128.7,128.1,127.5,119.6,115.0,113.6,75.8$, $70.0,60.7,41.9,31.1,27.3,22.3,21.0,13.9$.<smiles>Cc1ccc(F)cc1C([18O])[13C](=O)[O-]</smiles>

1-(5-fluoro-2-methylphenyl)-1-(methylsulfonamido)hexan-2-yl acetate (15): $10 \% \mathrm{Mn}_{2}(\mathrm{CO})_{10}$ used. Imine S15 (43.0 mg, $0.2 \mathrm{mmol}$ ) was subjected to GP3 and upon column chromatography (15 - 25\% ethyl acetate/hexanes) provided 70\% 15 (9:1 d.r.) as a colorless oil. $\mathrm{R}_{\mathrm{f}}$ : 0.41 (30\% ethyl acetate/hexanes). IR (film) $\mathrm{cm}^{-1}: 3283,2957,2932,2872,1741,1320,1230,1153$. HRMS (ESITOF) $\mathrm{m} / \mathrm{z}$ : calc'd for $[\mathrm{M}+\mathrm{Na}]^{+} 368.1308$, found 368.1312. ${ }^{1} \mathrm{H} \mathrm{NMR}\left(400 \mathrm{MHz}, \mathrm{CDCl}_{3}\right) \delta: 7.16$ $(\mathrm{dd}, J=5.8 .8 .4 \mathrm{~Hz}, 1 \mathrm{H}), 7.04(\mathrm{dd}, J=2.7,1.9 \mathrm{~Hz}, 1 \mathrm{H}), 6.93(\mathrm{dd}, J=2.8,8.3 \mathrm{~Hz}, 1 \mathrm{H}), 5.06-5.10$ $(\mathrm{m}, 1 \mathrm{H}), 4.97-5.00(\mathrm{~m}, 1 \mathrm{H}), 2.66(\mathrm{~s}, 3 \mathrm{H}), 2.42(\mathrm{~s}, 3 \mathrm{H}), 2.05(\mathrm{~s}, 3 \mathrm{H}), 1.46-1.58(\mathrm{~m}, 2 \mathrm{H}), 1.16-$ $1.34(\mathrm{~m}, 4 \mathrm{H}), 0.84(\mathrm{t}, J=7.0 \mathrm{~Hz}, 3 \mathrm{H}) \cdot{ }^{13} \mathrm{C} \mathrm{NMR}\left(100 \mathrm{MHz}, \mathrm{CDCl}_{3}\right) \delta: 170.8,161.5\left(\mathrm{~d},{ }^{1} J_{\mathrm{CF}}=\right.$ $244.5 \mathrm{~Hz}), 138.0\left(\mathrm{~d},{ }^{3} J_{\mathrm{CF}}=6.3 \mathrm{~Hz}\right), 132.4\left(\mathrm{~d},{ }^{3} J_{\mathrm{CF}}=7.7 \mathrm{~Hz}\right), 132.0\left(\mathrm{~d},{ }^{4} J_{\mathrm{CF}}=3.2 \mathrm{~Hz}\right), 115.2(\mathrm{~d}$, $\left.{ }^{2} J_{\mathrm{CF}}=20.9 \mathrm{~Hz}\right), 114.1\left(\mathrm{~d},{ }^{2} J_{\mathrm{CF}}=22.4 \mathrm{~Hz}\right), 74.9,56.0\left(\mathrm{~d},{ }^{4} J_{\mathrm{CF}}=1.5 \mathrm{~Hz}\right), 42.2,29.4,27.6,22.4$, 21.0, 18.9, 14.0. ${ }^{19} \mathrm{~F}\left(376 \mathrm{MHz}, \mathrm{CDCl}_{3}\right) \delta:-115.6$. 


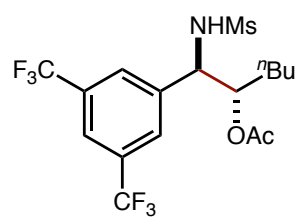

1-(3,5-bis(trifluoromethyl)phenyl)-1-(methylsulfonamido)hexan-2-yl acetate (16): Imine S16 (63.8 $\mathrm{mg}, 0.2 \mathrm{mmol}$ ) was subjected to GP3 and upon column chromatography (15 - 25\% ethyl acetate/hexanes) provided $71 \% \mathbf{1 6}\left(1: 1 \mathrm{~d} . \mathrm{r}\right.$.) as a yellow oil. $\mathrm{R}_{\mathrm{f}}$ : 0.50 (30\% ethyl acetate/hexanes). IR (film) $\mathrm{cm}^{-1}$ : 3282, 2960, 2935, 2866, 1376, 1279, 1134. HRMS (ESI-TOF) m/z: calc'd for $[\mathrm{M}+\mathrm{Na}]^{+}$472.0993, found 472.1035. IR (film) $\mathrm{cm}^{-1}:$ 3282, 2960, 2935, 2866, 1376, 1279, 1134.

Major diastereomer: ${ }^{1} \mathrm{H} \mathrm{NMR}\left(400 \mathrm{MHz}, \mathrm{CDCl}_{3}\right) \delta: 7.87(\mathrm{~s}, 1 \mathrm{H}), 7.79(\mathrm{~s}, 2 \mathrm{H}), 5.63(\mathrm{~d}, J=5.9$ $\mathrm{Hz}, 1 \mathrm{H}), 5.12-5.16(\mathrm{~m}, 1 \mathrm{H}), 4.86(\mathrm{dd}, J=3.7,6.9 \mathrm{~Hz}, 1 \mathrm{H}), 2.77(\mathrm{~s}, 3 \mathrm{H}), 2.07(\mathrm{~s}, 3 \mathrm{H}), 1.57-$ $1.73(\mathrm{~m}, 1 \mathrm{H}), 1.40-1.45(\mathrm{~m}, 4 \mathrm{H}), 1.24-1.36(\mathrm{~m}, 3 \mathrm{H}) .{ }^{13} \mathrm{C} \mathrm{NMR}\left(100 \mathrm{MHz}, \mathrm{CDCl}_{3}\right) \delta: 171.2$, 140.3, 131.6 - 133.0 (m), 128.1, $123.2\left(\mathrm{q},{ }^{1} J_{\mathrm{CF}}=272.8 \mathrm{~Hz}\right), 122.4-122.5(\mathrm{~m}), 75.7,59.5,42.3$, $30.4,27.5,22.3,20.9,13.9 .{ }^{19} \mathrm{~F}\left(376 \mathrm{MHz}, \mathrm{CDCl}_{3}\right) \delta:-62.9$.

Minor diastereomer: ${ }^{1} \mathrm{H}$ NMR $\left(400 \mathrm{MHz}, \mathrm{CDCl}_{3}\right) \delta: 7.85(\mathrm{~s}, 1 \mathrm{H}), 7.77(\mathrm{~s}, 2 \mathrm{H}), 5.51(\mathrm{~d}, J=8.0$ $\mathrm{Hz}, 1 \mathrm{H}), 5.04-5.08(\mathrm{~m}, 1 \mathrm{H}), 4.78(\mathrm{dd}, J=4.2,8.3 \mathrm{~Hz}, 1 \mathrm{H}), 2.77(\mathrm{~s}, 3 \mathrm{H}), 2.03(\mathrm{~s}, 3 \mathrm{H}), 1.57-$ $1.73(\mathrm{~m}, 1 \mathrm{H}), 1.40-1.45(\mathrm{~m}, 4 \mathrm{H}), 1.24-1.36(\mathrm{~m}, 3 \mathrm{H}) .{ }^{13} \mathrm{C} \mathrm{NMR}\left(100 \mathrm{MHz}, \mathrm{CDCl}_{3}\right) \delta: 170.4$, 142.1, $131.6-133.0(\mathrm{~m}), 127.2,123.1\left(\mathrm{q},{ }^{1} J_{\mathrm{CF}}=273.7 \mathrm{~Hz}\right), 122.4-122.5(\mathrm{~m}), 75.8,59.5,42.4$, 31.3, 27.4, 22.4, 20.7, 13.9. ${ }^{19} \mathrm{~F}\left(376 \mathrm{MHz} \mathrm{CDCl}_{3}\right) \delta:-62.9$.<smiles>O=C(O)C(c1ccc(F)nc1F)C([18O])[18O]</smiles>

1-(2,6-difluoropyridin-3-yl)-1-(methylsulfonamido)hexan-2-yl acetate (17): Imine S17 (44.0 $\mathrm{mg}, 0.2 \mathrm{mmol})$ was subjected to GP3 using $10 \% \mathrm{Mn}_{2}(\mathrm{CO})_{10}$ catalyst and upon column chromatography (20 - 50\% ethyl acetate/hexanes) provided 91\% 17 (1.4:1 d.r.) as a white solid. $R_{\mathrm{f}}: 0.31,0.42$ (50\% ethyl acetate/hexanes). IR (film): 3282, 2959, 2932, 2862, 1740, 1610, 1468. HRMS (ESI-TOF) m/z: calc'd for [M+Na] 373.1010, found 373.0997.

Major diastereomer: ${ }^{1} \mathrm{H} \mathrm{NMR}\left(400 \mathrm{MHz}, \mathrm{CDCl}_{3}\right) \delta: 7.84-7.93(\mathrm{~m}, 1 \mathrm{H}), 6.85-6.90(\mathrm{~m}, 1 \mathrm{H})$, $5.42(\mathrm{~d}, J=8.9 \mathrm{~Hz}, 1 \mathrm{H}), 5.12(\mathrm{td}, J=4.8,10.1 \mathrm{~Hz}, 1 \mathrm{H}), 4.82(\mathrm{dd}, J=5.0,9.0 \mathrm{~Hz}, 1 \mathrm{H}), 2.84(\mathrm{~s}$, $3 \mathrm{H}), 2.05(\mathrm{~s}, 3 \mathrm{H}), 1.53-1.67(\mathrm{~m}, 2 \mathrm{H}), 1.27-1.34(\mathrm{~m}, 4 \mathrm{H}), 0.85-0.89(\mathrm{~m}, 3 \mathrm{H}) .{ }^{13} \mathrm{C}$ NMR $(100$ $\left.\mathrm{MHz}, \mathrm{CDCl}_{3}\right) \delta: 170.6,161.2\left(\mathrm{dd},{ }^{1,3} J_{\mathrm{CF}}=14.4,248.4 \mathrm{~Hz}\right), 158.3\left(\mathrm{dd},{ }^{1,3} J_{\mathrm{CF}}=14.3,246.0 \mathrm{~Hz}\right)$, $143.6\left(\mathrm{dd},{ }^{3,3} J_{\mathrm{CF}}=4.6,7.8 \mathrm{~Hz}\right), 118.1\left(\mathrm{dd},{ }^{2,4} J_{\mathrm{CF}}=6.2,26.2 \mathrm{~Hz}\right), 106.9\left(\mathrm{dd},{ }^{2,4} J_{\mathrm{CF}}=5.4,34.5 \mathrm{~Hz}\right)$, $74.7,54.3\left(\mathrm{~d},{ }^{4} J_{\mathrm{CF}}=2.8 \mathrm{~Hz}\right), 42.0,31.0,27.3,22.4,20.9,13.9 .{ }^{19} \mathrm{~F}\left(376 \mathrm{MHz}, \mathrm{CDCl}_{3}\right) \delta:-68.0,-$ 70.3 .

Minor diastereomer: ${ }^{1} \mathrm{H}$ NMR $\left(400 \mathrm{MHz}, \mathrm{CDCl}_{3}\right) \delta: 7.84-7.93(\mathrm{~m}, 1 \mathrm{H}), 6.85-6.90(\mathrm{~m}, 1 \mathrm{H})$, $5.61(\mathrm{~d}, J=8.2 \mathrm{~Hz}, 1 \mathrm{H}), 5.18(\mathrm{td}, J=4.4,8.8 \mathrm{~Hz}, 1 \mathrm{H}), 4.87(\mathrm{dd}, J=4.4,8.3 \mathrm{~Hz}, 1 \mathrm{H}), 2.83(\mathrm{~s}, 3 \mathrm{H})$, $2.04(\mathrm{~s}, 3 \mathrm{H}), 1.53-1.67(\mathrm{~m}, 2 \mathrm{H}), 1.27-1.34(\mathrm{~m}, 4 \mathrm{H}), 0.85-0.89(\mathrm{~m}, 3 \mathrm{H}) .{ }^{13} \mathrm{C} \mathrm{NMR}(100 \mathrm{MHz}$, $\left.\mathrm{CDCl}_{3}\right) \delta: 170.9,161.2\left(\mathrm{dd},{ }^{1,3} J_{\mathrm{CF}}=14.4,248.6 \mathrm{~Hz}\right), 158.7\left(\mathrm{dd},{ }^{1,3} J_{\mathrm{CF}}=14.1,245.5 \mathrm{~Hz}\right), 145.0(\mathrm{dd}$, $\left.{ }^{3,3} J_{\mathrm{CF}}=4.5,7.8 \mathrm{~Hz}\right), 116.5\left(\mathrm{dd},{ }^{2,4} J_{\mathrm{CF}}=6.3,25.7 \mathrm{~Hz}\right), 106.9\left(\mathrm{dd},{ }^{2,4} J_{\mathrm{CF}}=4.8,34.4 \mathrm{~Hz}\right), 75.4,53.9$ $\left(\mathrm{d},{ }^{4} J_{\mathrm{CF}}=2.8 \mathrm{~Hz}\right), 41.9,30.9,27.5,22.4,21.0,13.9 .{ }^{19} \mathrm{~F}\left(376 \mathrm{MHz}, \mathrm{CDCl}_{3}\right) \delta:-67.7,-69.9$. 
<smiles>COC(O)C([NH3+])CCCc1ccccc1</smiles>

4-((4-methylphenyl)sulfonamido)-1-phenylnonan-5-yl acetate (18): : To the $\alpha$ oxy iodide reaction described in GP3, Imine $\mathbf{S 1 8}(60.2 \mathrm{mg}, 0.2 \mathrm{mmol}), \mathrm{Zn}$ (26.2 $\mathrm{mg}, 0.4 \mathrm{mmol})$, Hantzsch ester (37.9 mg, $0.15 \mathrm{mmol})$, and $\mathrm{Mn}_{2}(\mathrm{CO})_{10}(7.8 \mathrm{mg}, 0.02 \mathrm{mmol})$ were added the same time. The reaction was degassed by stirring at $-78^{\circ} \mathrm{C}$ for 20 min under vacuum. The reaction was irradiated with Blue LEDs $(34 \mathrm{~W})$. Upon column chromatography $(10 \%$ ethyl acetate/hexanes) provides 55\% 18 (1:1 d.r.) as colorless oil. $\mathrm{R}_{\mathrm{f}}$ : 0.57 (30\% ethyl acetate/hexanes). IR (film): 3280 , 3005, 2988, 2360, 1735, 1453, 1326, 1275, 1260, 1159, 1092, 749, 701, 548. HRMS (ESI-TOF) $\mathrm{m} / \mathrm{z}$ : calc'd for $[\mathrm{M}+\mathrm{Na}]^{+} 454.2028$, found 454.2057 .

Major diastereomer: ${ }^{1} \mathrm{H}$ NMR $\left(400 \mathrm{MHz}, \mathrm{CDCl}_{3}\right) \delta: 7.71-7.73(\mathrm{~m}, 2 \mathrm{H}), 7.14-7.27(\mathrm{~m}, 5 \mathrm{H})$, $7.03-7.08(\mathrm{~m}, 2 \mathrm{H}), 4.81-4.85(\mathrm{~m}, 1 \mathrm{H}), 4.54(\mathrm{~d}, J=9.4 \mathrm{~Hz}, 1 \mathrm{H}), 3.35-3.44(\mathrm{~m}, 1 \mathrm{H}), 2.45-$ $2.54(\mathrm{~m}, 2 \mathrm{H}), 2.39(\mathrm{~s}, 3 \mathrm{H}), 1.95(\mathrm{~s}, 3 \mathrm{H}), 1.25-1.50(\mathrm{~m}, 6 \mathrm{H}), 1.05-1.19(\mathrm{~m}, 4 \mathrm{H}), 0.78-0.84(\mathrm{~m}$, $3 \mathrm{H}) .{ }^{13} \mathrm{C} \mathrm{NMR}\left(150 \mathrm{MHz}, \mathrm{CDCl}_{3}\right) \delta: 170.4,143.2,141.6,138.5,129.5,128.3,128.2,126.9,125.8$, 74.3, 55.9, 35.3, 30.9, 30.0, 27.4, 27.1, 22.3, 21.4, 20.8, 13.8 .

Minor diastereomer: ${ }^{1} \mathrm{H} \mathrm{NMR}\left(400 \mathrm{MHz}, \mathrm{CDCl}_{3}\right) \delta$ : $7.71-7.73(\mathrm{~m}, 2 \mathrm{H}), 7.14-7.27(\mathrm{~m}, 5 \mathrm{H})$, $7.03-7.08(\mathrm{~m}, 2 \mathrm{H}), 4.72(\mathrm{~d}, J=9.3 \mathrm{~Hz}, 1 \mathrm{H}), 4.47-4.51(\mathrm{~m}, 1 \mathrm{H}), 3.35-3.44(\mathrm{~m}, 1 \mathrm{H}), 2.45-$ $2.54(\mathrm{~m}, 2 \mathrm{H}), 2.38(\mathrm{~s}, 3 \mathrm{H}), 1.95(\mathrm{~s}, 3 \mathrm{H}), 1.25-1.50(\mathrm{~m}, 6 \mathrm{H}), 1.05-1.19(\mathrm{~m}, 4 \mathrm{H}), 0.78-0.84(\mathrm{~m}$, $3 \mathrm{H}) .{ }^{13} \mathrm{C} \mathrm{NMR}\left(150 \mathrm{MHz}, \mathrm{CDCl}_{3}\right) \delta: 171.2,143.3,141.6,138.1,129.6,128.3,128.2,127.1,125.8$, $76.1,55.9,35.2,32.7,29.7,27.5,27.1,22.2,21.4,20.9,13.8$.

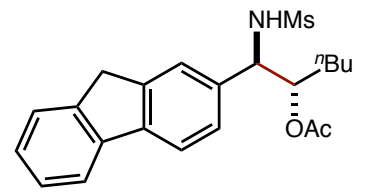

1-(9H-fluoren-2-yl)-1-(methylsulfonamido)hexan-2-yl acetate (19): Imine S19 (54.3 mg, 0.2 mmol) was subjected to GP3 using $10 \% \mathrm{Mn}_{2}(\mathrm{CO})_{10}$ catalyst and upon column chromatography (15 $-20 \%$ ethyl acetate/hexanes) provided 55\% 19 (3:1 d.r.) as an off-white solid. Rf: 0.32 (30\% ethyl acetate/hexanes). IR (film): 3281, 3050, 2959, 1735, 1264. HRMS (ESI-TOF) m/z: calc'd for $[\mathrm{M}+\mathrm{Na}]^{+}$424.1558, found 424.1566.

Major diastereomer: ${ }^{1} \mathrm{H}$ NMR $\left(400 \mathrm{MHz} \mathrm{CDCl}_{3}\right) \delta: 7.78(\mathrm{~d}, J=7.7 \mathrm{~Hz}, 1 \mathrm{H}), 7.56(\mathrm{~d}, J=7.4$ $\mathrm{Hz}, 1 \mathrm{H}), 7.49(\mathrm{~d}, J=8.3 \mathrm{~Hz}, 1 \mathrm{H}), 7.39(\mathrm{t}, J=7.2 \mathrm{~Hz}, 1 \mathrm{H}), 7.29-7.35(\mathrm{~m}, 2 \mathrm{H}), 5.14-5.20(\mathrm{~m}$, $2 \mathrm{H}), 4.77(\mathrm{dd}, J=4.4,7.8 \mathrm{~Hz}, 1 \mathrm{H}), 3.92(\mathrm{~s}, 2 \mathrm{H}), 2.65(\mathrm{~s}, 3 \mathrm{H}), 2.06,(\mathrm{~s}, 3 \mathrm{H}), 1.51-1.58(\mathrm{~m}, 2 \mathrm{H})$, $1.23-1.35(\mathrm{~m}, 4 \mathrm{H}), 0.82-0.86(\mathrm{~m}, 3 \mathrm{H}) .{ }^{13} \mathrm{C} \mathrm{NMR}\left(100 \mathrm{MHz}, \mathrm{CDCl}_{3}\right) \delta: 171.1,143.9,143.5$, $142.1,141.1,135.8,127.3,127.1,126.5,125.3,124.5,120.6,120.3,76.3,60.4,42.1,37.1,30.1$, 27.7, 22.5, 21.2, 14.0.

Minor diastereomer: ${ }^{1} \mathrm{H} \mathrm{NMR}\left(400 \mathrm{MHz}, \mathrm{CDCl}_{3}\right) \delta: 7.78(\mathrm{~d}, J=7.7 \mathrm{~Hz}, 1 \mathrm{H}), 7.56(\mathrm{~d}, J=7.4$ $\mathrm{Hz}, 1 \mathrm{H}), 7.49$ (d, $J=8.3 \mathrm{~Hz}, 1 \mathrm{H}), 7.39(\mathrm{t}, J=7.2 \mathrm{~Hz}, 1 \mathrm{H}), 7.29-7.35(\mathrm{~m}, 2 \mathrm{H}), 5.14-5.20(\mathrm{~m}$, 2H), $4.61(\mathrm{dd}, J=6.7,8.4 \mathrm{~Hz}, 1 \mathrm{H}), 3.92(\mathrm{~s}, 2 \mathrm{H}), 2.57(\mathrm{~s}, 3 \mathrm{H}), 2.11,(\mathrm{~s}, 3 \mathrm{H}), 1.51-1.58(\mathrm{~m}, 2 \mathrm{H})$, $1.23-1.35(\mathrm{~m}, 4 \mathrm{H}), 0.82-0.86(\mathrm{~m}, 3 \mathrm{H}) .{ }^{13} \mathrm{C}$ NMR $\left(100 \mathrm{MHz}, \mathrm{CDCl}_{3}\right) \delta: 171.4,144.3,143.4$, $142.3,141.0,137.1,127.3,127.1,125.8,125.3,123.9,120.5,120.2,76.1,61.2,42.2,37.0,31.3$, $27.4,22.4,21.2,14.0$. 


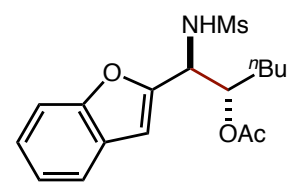

1-(benzofuran-2-yl)-1-(methylsulfonamido)hexan-2-yl acetate (20): Imine S20 (44.6 mg, 0.2 mmol) was subjected to GP3 and upon column chromatography (15\% ethyl acetate/hexanes) provided 52\% 20 (2:1 d.r.) as colorless oil. $\mathrm{R}_{\mathrm{f}}$ : 0.41 (30\% ethyl acetate/hexanes). IR (film): 3275 , 3005, 2958, 2360, 2341, 1740, 1454, 1323, 1275, 1260, 1155, 980, 750, 523. HRMS (ESI-TOF) $\mathrm{m} / \mathrm{z}$ : calc'd for $[\mathrm{M}+\mathrm{Na}]+376.1195$, found 376.1177 .

Major diastereomer: ${ }^{1} \mathrm{H}$ NMR $\left(400 \mathrm{MHz}, \mathrm{CDCl}_{3}\right) \delta$ : 7.54-7.57 (m, 1H), 7.44-7.48 (m, 1H), 7.22$7.33(\mathrm{~m}, 2 \mathrm{H}), 6.72(\mathrm{~s}, 1 \mathrm{H}), 5.33-5.37(\mathrm{~m}, 1 \mathrm{H}), 5.31(\mathrm{~d}, J=9.3 \mathrm{~Hz}, 1 \mathrm{H}), 4.91(\mathrm{dd}, J=4.3,9.3 \mathrm{~Hz}$, $1 \mathrm{H}), 2.80(\mathrm{~s}, 3 \mathrm{H}), 2.05(\mathrm{~s}, 3 \mathrm{H}), 1.62-1.68(\mathrm{~m}, 2 \mathrm{H}), 1.26-1.37(\mathrm{~m}, 4 \mathrm{H}), 0.85-0.89(\mathrm{~m}, 3 \mathrm{H}) .{ }^{13} \mathrm{C} \mathrm{NMR}$ $\left(150 \mathrm{MHz}, \mathrm{CDCl}_{3}\right) \delta: 171.0,154.9,152.8,127.7,124.9,123.4,121.4,111.4,105.6,75.1,54.8$, 41.8, 30.3, 27.5, 22.4, 21.1, 14.0.

Minor diastereomer: ${ }^{1} \mathrm{H}$ NMR $\left(400 \mathrm{MHz}, \mathrm{CDCl}_{3}\right) \delta$ : 7.54-7.57 (m, 1H), 7.44-7.48 (m, 1H), 7.22$7.33(\mathrm{~m}, 2 \mathrm{H}), 6.69(\mathrm{~s}, 1 \mathrm{H}), 5.16-5.21(\mathrm{~m}, 1 \mathrm{H}), 5.14(\mathrm{~d}, J=9.5 \mathrm{~Hz}, 1 \mathrm{H}), 4.78(\mathrm{dd}, J=5.3,9.5 \mathrm{~Hz}$, $1 \mathrm{H}), 2.80(\mathrm{~s}, 3 \mathrm{H}), 2.09(\mathrm{~s}, 3 \mathrm{H}), 1.62-1.68(\mathrm{~m}, 2 \mathrm{H}), 1.26-1.37(\mathrm{~m}, 4 \mathrm{H}), 0.85-0.89(\mathrm{~m}, 3 \mathrm{H}) .{ }^{13} \mathrm{C} \mathrm{NMR}$ $\left(150 \mathrm{MHz}, \mathrm{CDCl}_{3}\right) \delta: 170.8,155.0,153.7,127.6,125.0,123.4,121.5,111.4,105.0,73.7,54.9$, $42.0,31.1,27.3,22.4,21.1,14.0$.

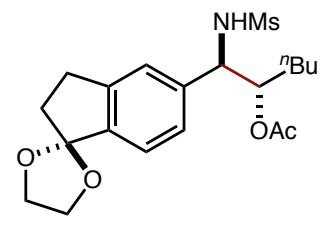

1-(2,3-dihydrospiro[indene-1,2'-[1,3] dioxolan]-5-yl)-1-(methylsulfonamido)hexan-2-yl acetate (21): Imine S21 (56.2 $\mathrm{mg}, 0.2 \mathrm{mmol})$ was subjected to GP3 and upon column chromatography ( $25 \%$ ethyl acetate/hexanes) provides $66 \% \mathbf{2 1}$ (3:1 d.r.) as colorless oil. Rf: 0.30 (30\% ethyl acetate/hexanes). IR (film): 3279, 2931, 2390, 1738, 1447, 1319, 1233, 1155, 1041, 980, 919. HRMS (ESI-TOF) m/z: calc'd for $[\mathrm{M}+\mathrm{Na}]^{+} 434.1613$, found 434.1625.

Major diastereomer: ${ }^{1} \mathrm{H}$ NMR $\left(400 \mathrm{MHz} \mathrm{CDCl}_{3}\right) \delta$ : 7.37-7.35 (m, 1H), 7.16-7.22 (m, 2H), 5.52 $(\mathrm{d}, J=8.4 \mathrm{~Hz}, 1 \mathrm{H}), 5.06-5.11(\mathrm{~m}, 1 \mathrm{H}), 4.71(\mathrm{dd}, J=4.0,8.4 \mathrm{~Hz}, 1 \mathrm{H}), 4.13-4.21(\mathrm{~m}, 2 \mathrm{H}), 4.06-$ $4.12(\mathrm{~m}, 2 \mathrm{H}), 2.93-2.96(\mathrm{~m}, 2 \mathrm{H}), 2.62(\mathrm{~s}, 3 \mathrm{H}), 2.29-2.32(\mathrm{~m}, 2 \mathrm{H}), 2.04(\mathrm{~s}, 3 \mathrm{H}), 1.39-1.46(\mathrm{~m}, 2 \mathrm{H})$, $1.18-1.30(\mathrm{~m}, 4 \mathrm{H}), 0.81-0.84(\mathrm{~m}, 3 \mathrm{H}) .{ }^{13} \mathrm{C} \mathrm{NMR}\left(150 \mathrm{MHz}, \mathrm{CDCl}_{3}\right) \delta: 171.1,144.5,142.2,138.7$, 126.4, 124.5, 123.6, 116.9, 76.3, 65.3, 65.3, 60.0, 42.1, 37.2, 29.7, 28.5, 27.7, 22.4, 21.1, 14.0.

Minor diastereomer: ${ }^{1} \mathrm{H}$ NMR $\left(400 \mathrm{MHz}, \mathrm{CDCl}_{3}\right) \delta: 7.37-7.35(\mathrm{~m}, 1 \mathrm{H}), 7.16-7.22(\mathrm{~m}, 2 \mathrm{H}), 5.38$ $(\mathrm{d}, J=8.8 \mathrm{~Hz}, 1 \mathrm{H}), 5.01-5.06(\mathrm{~m}, 1 \mathrm{H}), 4.52(\mathrm{dd}, J=6.6,8.8 \mathrm{~Hz}, 1 \mathrm{H}), 4.13-4.21(\mathrm{~m}, 2 \mathrm{H}), 4.06-$ $4.12(\mathrm{~m}, 2 \mathrm{H}), 2.93-2.96(\mathrm{~m}, 2 \mathrm{H}), 2.55(\mathrm{~s}, 3 \mathrm{H}), 2.29-2.32(\mathrm{~m}, 2 \mathrm{H}), 2.07(\mathrm{~s}, 3 \mathrm{H}), 1.39-1.46(\mathrm{~m}, 2 \mathrm{H})$, 1.18-1.30 (m, 4H), 0.81-0.84 (m, 3H). $\left.{ }^{13} \mathrm{C} \mathrm{NMR} \mathrm{(150} \mathrm{MHz,} \mathrm{CDCl}_{3}\right) \delta: 171.3,144.9,142.4,140.3$, 125.8, 124.0, 123.9, 116.8, 75.9, 65.3, 65.3, 60.8, 42.2, 37.2, 31.1, 28.5, 27.4, 22.4, 21.1, 14.0. 
<smiles>N#Cc1ccc(C(N)C(Br)(Br)c2ccccc2)c([N+](=O)[O-])c1</smiles>

1-(4-cyano-2-methylphenyl)-1-(methylsulfonamido)hexan-2-yl acetate (22): Imine S22 (44.5 $\mathrm{mg}, 0.2 \mathrm{mmol}$ ) was subjected to GP3 using $10 \% \mathrm{Mn}_{2}(\mathrm{CO})_{10}$ catalyst and upon column chromatography (15 - 25\% ethyl acetate/hexanes) provided 61\% 22 (3:1 d.r.) as a colorless oil. $\mathrm{R}_{\mathrm{f}}$ : 0.24 (30\% ethyl acetate/hexanes). IR (film): 3282, 2958, 2931, 2871, 1736, 1319, 1153. HRMS (ESI-TOF) $\mathrm{m} / \mathrm{z}$ : calc'd for [M+Na] ${ }^{+} 375.1354$, found 375.1340 .

Major diastereomer: ${ }^{1} \mathrm{H}$ NMR $\left(400 \mathrm{MHz}, \mathrm{CDCl}_{3}\right) \delta: 7.50-7.55(\mathrm{~m}, 2 \mathrm{H}), 7.45(\mathrm{~d}, J=8.1 \mathrm{~Hz}$, 1H), $5.14(\mathrm{~d}, J=6.4 \mathrm{~Hz}, 1 \mathrm{H}), 5.03-5.09(\mathrm{~m}, 2 \mathrm{H}), 2.69$ (s, 3H), $2.51(\mathrm{~s}, 3 \mathrm{H}), 2.04$, (s, 3H), $1.46-$ $1.52(\mathrm{~m}, 2 \mathrm{H}), 1.17-1.33(\mathrm{~m}, 4 \mathrm{H}), 0.84(\mathrm{t}, J=7.0 \mathrm{~Hz}, 3 \mathrm{H}) .{ }^{13} \mathrm{C} \mathrm{NMR}\left(100 \mathrm{MHz}, \mathrm{CDCl}_{3}\right) \delta: 170.6$, 141.8, 137.9, 134.3, 130.1, 128.2, 118.5, 112.1, 74.6, 55.8, 42.1, 29.5, 27.5, 22.4, 21.0, 19.5, 13.9. Minor diastereomer: ${ }^{1} \mathrm{H} \mathrm{NMR}\left(400 \mathrm{MHz}^{\mathrm{CDCl}} \mathrm{CD}_{3}\right) \delta: 7.39-7.54(\mathrm{~m}, 3 \mathrm{H}), 5.45(\mathrm{~d}, J=8.3 \mathrm{~Hz}$, $1 \mathrm{H}), 4.99-5.10(\mathrm{~m}, 1 \mathrm{H}), 4.93(\mathrm{dd}, J=4.9,8.3 \mathrm{~Hz}), 2.65(\mathrm{~s}, 3 \mathrm{H}), 2.46(\mathrm{~s}, 3 \mathrm{H}), 2.04,(\mathrm{~s}, 3 \mathrm{H}), 1.49$ $-1.52(\mathrm{~m}, 2 \mathrm{H}), 1.26-1.33(\mathrm{~m}, 4 \mathrm{H}), 0.82-0.89(\mathrm{~m}, 3 \mathrm{H}) .{ }^{13} \mathrm{C} \mathrm{NMR}\left(100 \mathrm{MHz}, \mathrm{CDCl}_{3}\right) \delta: 170.7$, 142.7, 136.7, 134.7, 130.3, 127.1, 118.4, 112.3, 74.9, 56.1, 42.4, 31.2, 27.5, 22.4, 20.9, 19.4, 14.0. HRMS (ESI-TOF) m/z: calc'd for [M+Na] 375.1354 , found 375.1340.

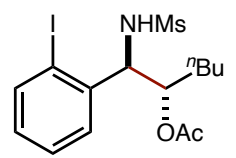

1-(2-iodophenyl)-1-(methylsulfonamido)hexan-2-yl acetate (23): Imine S23 (61.8 mg, 0.2 mmol) was subjected to GP3 and upon column chromatography (15\% ethyl acetate/hexanes) provides $56 \% \mathbf{2 3}$ (2:1 d.r.) as a yellow oil. IR (film): 3276, 2956, 2930, 2860, 1739, 1320, 1227 , 1154, 1010, 977, 756, 730, 518. HRMS (ESI-TOF) m/z: calc'd for $[\mathrm{M}+\mathrm{H}]^{+} 440.0392$, found 440.0382 .

Major diastereomer: $\mathrm{R}_{\mathrm{f}}: 0.35$ (30\% ethyl acetate/hexanes) ${ }^{1} \mathrm{H}$ NMR $\left(400 \mathrm{MHz}, \mathrm{CDCl}_{3}\right) \delta: 7.88$ $(\mathrm{dd}, J=1.7,7.9 \mathrm{~Hz}, 1 \mathrm{H}), 7.36(\mathrm{~m}, 2 \mathrm{H}), 7.01(\mathrm{~m}, 1 \mathrm{H}), 5.37(\mathrm{~d}, J=8.2 \mathrm{~Hz}, 1 \mathrm{H}), 5.23(\mathrm{~m}, 1 \mathrm{H}), 5.04$ $(\mathrm{dd}, J=6.1,8.2 \mathrm{~Hz}, 1 \mathrm{H}), 2.66(\mathrm{~s}, 3 \mathrm{H}), 1.96(\mathrm{~s}, 3 \mathrm{H}), 1.74(\mathrm{~m}, 1 \mathrm{H}), 1.49(\mathrm{~m}, 1 \mathrm{H}), 1.27(\mathrm{~m}, 4 \mathrm{H})$, $0.86(\mathrm{t}, J=7.4 \mathrm{~Hz}, 3 \mathrm{H}) .{ }^{13} \mathrm{C} \mathrm{NMR}\left(150 \mathrm{MHz}, \mathrm{CDCl}_{3}\right) \delta: 170.4,140.4,140.2,130.1,128.9,128.8$, 75.2, 63.3, 41.9, 30.7, 27.5, 22.4, 20.9, 14.0.

Minor diastereomer: $\mathrm{R}_{\mathrm{f}}: 0.25$ (30\% ethyl acetate/hexanes) ${ }^{1} \mathrm{H}$ NMR $\left(400 \mathrm{MHz}, \mathrm{CDCl}_{3}\right) \delta: 7.88$ $(\mathrm{dd}, J=1.2,8.0 \mathrm{~Hz}, 1 \mathrm{H}), 7.36(\mathrm{dt}, J=1.2,11.3 \mathrm{~Hz}, 1 \mathrm{H}), 7.25(\mathrm{~m}, 2 \mathrm{H}), 7.01(\mathrm{~m}, 1 \mathrm{H}), 5.38(\mathrm{~d}, J=$ $8.5 \mathrm{~Hz}, 1 \mathrm{H}), 5.12(\mathrm{~s}, 1 \mathrm{H}), 5.00(\mathrm{~m}, 1 \mathrm{H}), 2.67(\mathrm{~s}, 3 \mathrm{H}), 2.01(\mathrm{~s}, 3 \mathrm{H}), 1.70(\mathrm{~m}, 2 \mathrm{H}), 1.35(\mathrm{~m}, 4 \mathrm{H})$, $0.89(\mathrm{t}, J=7.2 \mathrm{~Hz}, 1 \mathrm{H}) .{ }^{13} \mathrm{C} \mathrm{NMR}\left(150 \mathrm{MHz}, \mathrm{CDCl}_{3}\right) \delta: 170.4,140.5,140.5,130.1,128.7,75.2$, 63.1, 42.2, 31.4, 27.5, 22.5, 20.9, 14.0. 
<smiles>CC(=O)OC(C(N)c1cccc(I)c1)C(C)(C)C</smiles>

1-(3-iodophenyl)-1-(methylsulfonamido)hexan-2-yl acetate (24): Imine S24 (61.8 mg, 0.2 mmol) was subjected to GP3 and upon column chromatography (15\% ethyl acetate/hexanes) provides $87 \% 24$ (2:1 d.r.) as colorless oil. $\mathrm{R}_{\mathrm{f}}$ : 0.42 (30\% ethyl acetate/hexanes). IR (film): 3279 , 2956, 2860, 2360, 1736, 1590, 1566, 1440, 1372, 1320, 1275, 1228, 1153, 978, 763, 750, 701, 519. HRMS (ESI-TOF) m/z: calc'd for $[\mathrm{M}+\mathrm{Na}]^{+} 462.0212$, found 462.0208 .

Major diastereomer: ${ }^{1} \mathrm{H} \mathrm{NMR}\left(400 \mathrm{MHz}, \mathrm{CDCl}_{3}\right) \delta$ : 7.65-7.69 (m, 2H), 7.27-7.31 (m, 1H), 7.09$7.13(\mathrm{~m}, 1 \mathrm{H}), 5.58(\mathrm{~d}, J=8.2 \mathrm{~Hz}, 1 \mathrm{H}), 5.06-5.11(\mathrm{~m}, 1 \mathrm{H}), 4.63(\mathrm{dd}, J=4.2,8.2 \mathrm{~Hz}, 1 \mathrm{H}), 2.69(\mathrm{~s}$, $3 \mathrm{H}), 2.05(\mathrm{~s}, 3 \mathrm{H}), 1.41-1.46(\mathrm{~m}, 2 \mathrm{H}), 1.20-1.31(\mathrm{~m}, 4 \mathrm{H}), 0.82-0.87(\mathrm{~m}, 3 \mathrm{H}) .{ }^{13} \mathrm{C} \mathrm{NMR}(150 \mathrm{MHz}$, $\left.\mathrm{CDCl}_{3}\right) \delta: 171.0,139.7,137.5,136.8,130.5,127.0,94.5,75.9,59.4,42.2,30.0,27.5,22.3,21.1$, 13.9.

Minor diastereomer: ${ }^{1} \mathrm{H}$ NMR $\left(400 \mathrm{MHz}, \mathrm{CDCl}_{3}\right) \delta$ : 7.65-7.69 (m, 2H), 7.27-7.31 (m, 1H), 7.09$7.13(\mathrm{~m}, 1 \mathrm{H}), 5.47(\mathrm{~d}, J=8.9 \mathrm{~Hz}, 1 \mathrm{H}), 5.00-5.05(\mathrm{~m}, 1 \mathrm{H}), 4.50(\mathrm{dd}, J=5.6,8.9 \mathrm{~Hz}, 1 \mathrm{H}), 2.64(\mathrm{~s}$, $3 \mathrm{H}), 2.07$ (s, 3H), 1.41-1.46 (m, 2H), 1.20-1.31 (m, 4H), 0.82-0.87 (m, 3H). ${ }^{13} \mathrm{C}$ NMR (150 MHz, $\left.\mathrm{CDCl}_{3}\right) \delta: 170.9,141.2,137.6,136.0,130.8,126.3,94.9,75.8,59.9,42.2,31.2,27.3,22.4,21.0$, 13.9 .<smiles>O=[N+]([O-])[C@@H]([18OH])c1ccc(I)cc1</smiles>

1-(4-iodophenyl)-1-(methylsulfonamido)hexan-2-yl acetate (25): Imine S25 (61.8 mg, 0.2 mmol) was subjected to GP3 and upon column chromatography (15\% ethyl acetate/hexanes) provides 83\% 25 (3:1 d.r.) as a yellow oil. IR (film): 3284, 2955, 2930, 1737, 1317, 1229, 1154 , 1006, 979, 732, 519. HRMS (ESI-TOF) m/z: calc'd for $[\mathrm{M}+\mathrm{H}]^{+} 440.0392$, found 440.0382 .

Major diastereomer: $\mathrm{R}_{\mathrm{f}}: 0.42$ (30\% ethyl acetate/hexanes) ${ }^{1} \mathrm{H}$ NMR $\left(400 \mathrm{MHz}, \mathrm{CDCl}_{3}\right) \delta: 7.71$ $(\mathrm{d}, J=8.4 \mathrm{~Hz}, 2 \mathrm{H}), 7.08(\mathrm{~d}, J=8.3 \mathrm{~Hz}, 2 \mathrm{H}), 5.49(\mathrm{~d}, J=7.9 \mathrm{~Hz}, 1 \mathrm{H}), 5.09(\mathrm{~m}, 1 \mathrm{H}), 4.64(\mathrm{dd}, J$ $=4.1,8.0 \mathrm{~Hz}, 1 \mathrm{H}), 2.66(\mathrm{~s}, 3 \mathrm{H}), 2.05(\mathrm{~s}, 3 \mathrm{H}), 1.41(\mathrm{~m}, 2 \mathrm{H}), 1.25(\mathrm{~m}, 4 \mathrm{H}), 0.84(\mathrm{t}, J=7.1 \mathrm{~Hz}, 1 \mathrm{H})$. ${ }^{13} \mathrm{C} \mathrm{NMR}\left(150 \mathrm{MHz} \mathrm{CDCl}_{3}\right) \delta: 171.1,137.9,137.0,129.7,94.1,75.9,59.7,42.1,30.0,27.6,22.4$, 21.1 .

Minor diastereomer: $\mathrm{R}_{\mathrm{f}}: 0.3\left(30 \%\right.$ ethyl acetate/hexanes) ${ }^{1} \mathrm{H}$ NMR $\left(400 \mathrm{MHz}, \mathrm{CDCl}_{3}\right) \delta: 7.71(\mathrm{~d}$, $J=8.4 \mathrm{~Hz}, 2 \mathrm{H}), 7.05(\mathrm{~d}, J=8.3 \mathrm{~Hz}, 2 \mathrm{H}), 5.38(\mathrm{~d}, J=8.6 \mathrm{~Hz}, 1 \mathrm{H}), 5.02(\mathrm{~m}, 1 \mathrm{H}), 4.51(\mathrm{dd}, J=$ 5.9, $8.6 \mathrm{~Hz}, 1 \mathrm{H}), 2.62(\mathrm{~s}, 3 \mathrm{H}), 2.06(\mathrm{~s}, 3 \mathrm{H}), 1.57(\mathrm{~m}, 1 \mathrm{H}), 1.49(\mathrm{~m}, 1 \mathrm{H}), 1.25(\mathrm{~m}, 4 \mathrm{H}), 0.84(\mathrm{t}, J=$ $7.1 \mathrm{~Hz}, 1 \mathrm{H}) .{ }^{13} \mathrm{C} \mathrm{NMR}\left(150 \mathrm{MHz}, \mathrm{CDCl}_{3}\right) \delta: 171.0,138.5,138.3,128.9,94.2,75.8,60.2,42.3$, $31.2,27.3,21.0,13.9$. 
<smiles>O=C(c1ccccc1)c1cccc(C([18O])[13C](=O)[O-])c1</smiles>

1-(3-benzoylphenyl)-1-(methylsulfonamido)hexan-2-yl acetate (26): Imine S26 (57.5 mg, 0.2 mmol) was subjected to GP3 using $10 \% \mathrm{Mn}_{2}(\mathrm{CO})_{10}$ catalyst and upon column chromatography (15 - 30\% ethyl acetate/hexanes) provided $68 \% 26$ (2:1 d.r.) as a white solid. $\mathrm{R}_{\mathrm{f}}: 0.26$ (30\% ethyl acetate/hexanes). IR (film): 3279, 3059, 2958, 2931, 2861, 1737, 1658, 1318, 733. HRMS (ESITOF) $\mathrm{m} / \mathrm{z}$ : calc'd for $[\mathrm{M}+\mathrm{Na}]^{+} 440.1508$, found 440.1523 .

Major diastereomer: ${ }^{1} \mathrm{H}$ NMR $\left(400 \mathrm{MHz} \mathrm{CDCl}_{3}\right) \delta: 7.22-7.80(\mathrm{~m}, 4 \mathrm{H}), 7.48-7.68(\mathrm{~m}, 5 \mathrm{H})$, $5.27(\mathrm{~d}, J=7.0 \mathrm{~Hz}, 1 \mathrm{H}), 5.13-5.18(\mathrm{~m}, 1 \mathrm{H}), 4.79(\mathrm{dd}, J=4.0,7.5 \mathrm{~Hz}, 1 \mathrm{H}), 2.71(\mathrm{~s}, 3 \mathrm{H}), 2.05(\mathrm{~s}$, $3 \mathrm{H}), 1.45-1.50(\mathrm{~m}, 1 \mathrm{H}), 1.21-1.33(\mathrm{~m}, 5 \mathrm{H}), 0.83-0.89(\mathrm{~m}, 3 \mathrm{H}) .{ }^{13} \mathrm{C} \mathrm{NMR}\left(100 \mathrm{MHz}, \mathrm{CDCl}_{3}\right)$ $\delta: 196.3,171.1,139.6,137.9,137.3,132.9,131.9,130.2,130.1,129.2,128.8,128.6,76.0,59.9$, 42.2, 30.2, 27.6, 22.4, 21.1, 14.0.

Minor diastereomer: ${ }^{1} \mathrm{H}$ NMR $\left(400 \mathrm{MHz}, \mathrm{CDCl}_{3}\right) \delta: 7.22-7.80(\mathrm{~m}, 4 \mathrm{H}), 7.48-7.68(\mathrm{~m}, 5 \mathrm{H})$, $5.25(\mathrm{~d}, J=8.2 \mathrm{~Hz}, 1 \mathrm{H}), 5.08-5.12(\mathrm{~m}, 1 \mathrm{H}), 4.68(\mathrm{dd}, J=5.2,8.6 \mathrm{~Hz}, 1 \mathrm{H}), 2.69(\mathrm{~s}, 3 \mathrm{H}), 2.05(\mathrm{~s}$, $3 \mathrm{H}), 1.45-1.50(\mathrm{~m}, 1 \mathrm{H}), 1.21-1.33(\mathrm{~m}, 5 \mathrm{H}), 0.83-0.89(\mathrm{~m}, 3 \mathrm{H}) .{ }^{13} \mathrm{C} \mathrm{NMR}\left(100 \mathrm{MHz}, \mathrm{CDCl}_{3}\right)$ $\delta: 196.3,170.8,138.4,138.1,137.3,132.9,131.0,130.3,130.1,129.1,128.6,128.3,76.1,60.2$, $42.3,31.3,27.4,22.4,21.0,14.0$. 
VIII. Synthesis and characterization of aza-pinacol products - aldehydes<smiles>CC(=O)OC(C)[C@H](N)c1ccccc1</smiles>

1-(methylsulfonamido)-1-phenylpropan-2-yl acetate (27): Acetaldehyde (40 $\mu \mathrm{L}, 0.6 \mathrm{mmol})$ was subjected to GP3 and upon column chromatography (15 - 25\% ethyl acetate/hexanes) provided 63\% 27 (3:1 d.r.) as a colorless oil. $\mathrm{R}_{\mathrm{f}}$ : 0.24 (30\% ethyl acetate/hexanes). IR (film): 3250 , 2981, 1734, 1236, 1044. HRMS (ESI-TOF) m/z: calc'd for [M+Na] ${ }^{+}$294.0076, found 294.0786.

Major diastereomer: ${ }^{1} \mathrm{H} \mathrm{NMR}\left(400 \mathrm{MHz}, \mathrm{CDCl}_{3}\right) \delta$ : $7.29-7.41(\mathrm{~m}, 5 \mathrm{H}), 5.34(\mathrm{~d}, J=6.4 \mathrm{~Hz}$, 1H), $5.21(\mathrm{dq}, J=4.4,6.5 \mathrm{~Hz}, 1 \mathrm{H}), 4.65$ (dd, $J=4.4,8.4 \mathrm{~Hz}, 1 \mathrm{H}), 2.66$ (s, 3H), 2.07 (s, 3H), 1.16 $(\mathrm{d}, J=6.5 \mathrm{~Hz}, 3 \mathrm{H}) .{ }^{13} \mathrm{C} \mathrm{NMR}\left(100 \mathrm{MHz}, \mathrm{CDCl}_{3}\right) \delta: 170.6,137.2,128.9,128.5,129.8,72.7,60.8$, 41.9, 21.3, 16.1 .

Minor diastereomer: ${ }^{1} \mathrm{H} \mathrm{NMR}\left(400 \mathrm{MHz}^{\left.\mathrm{CDCl}_{3}\right)} \delta: 7.29-7.41(\mathrm{~m}, 5 \mathrm{H}), 5.27(\mathrm{~d}, J=6.8 \mathrm{~Hz}\right.$, 1H), 5.14 (quin., $J=6.3 \mathrm{~Hz}, 1 \mathrm{H}), 4.49$ (dd, $J=6.4,8.1 \mathrm{~Hz}, 1 \mathrm{H}), 2.58$ (s, 3H), 2.05 (s, 3H), 1.22 $(\mathrm{d}, J=6.4 \mathrm{~Hz}, 3 \mathrm{H}) .{ }^{13} \mathrm{C} \mathrm{NMR}\left(100 \mathrm{MHz}, \mathrm{CDCl}_{3}\right) \delta: 170.8,138.5,129.2,128.6,127.2,72.7,62.2$, 42.0, 21.2, 17.8 .

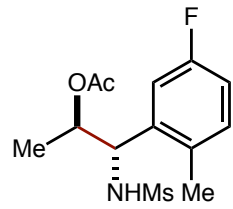

1-(5-fluoro-2-methylphenyl)-1-(methylsulfonamido)propan-2-yl acetate (28): Acetaldehyde (40 $\mu \mathrm{L}, 0.6 \mathrm{mmol}$ ) was subjected to GP3 using $10 \% \mathrm{Mn}_{2}(\mathrm{CO})_{10}$ and upon column chromatography (15 - 25\% ethyl acetate/hexanes) provided $60 \% 28$ (10:1 d.r.) as a colorless oil. $\mathrm{R}_{\mathrm{f}}: 0.33(30 \%$ ethyl acetate/hexanes). IR (film): 3282, 2980, 1738, 1238, 1153, 1063. HRMS (ESI-TOF) m/z: calc'd for $[\mathrm{M}+\mathrm{Na}]^{+}$326.0838, found 326.0842. ${ }^{1} \mathrm{H} \mathrm{NMR}\left(400 \mathrm{MHz}, \mathrm{CDCl}_{3}\right) \delta: 7.17(\mathrm{dd}, J=5.8$, $8.4 \mathrm{~Hz}, 1 \mathrm{H}), 7.05(\mathrm{dd}, J=2.7,4.4,6.5 \mathrm{~Hz}, 1 \mathrm{H}), 6.93(\mathrm{dt}, J=2.7,8.3 \mathrm{~Hz}, 1 \mathrm{H}), 5.16(\mathrm{dq}, J=4.4$, $6.5 \mathrm{~Hz}, 1 \mathrm{H}), 4.96-5.09(\mathrm{~m}, 1 \mathrm{H}), 2.68(\mathrm{~s}, 3 \mathrm{H}), 2.42(\mathrm{~s}, 3 \mathrm{H}), 2.08(\mathrm{~s}, 3 \mathrm{H}), 1.18(\mathrm{~d}, J=6.5 \mathrm{~Hz}, 3 \mathrm{H})$. ${ }^{13} \mathrm{C} \mathrm{NMR}\left(100 \mathrm{MHz}, \mathrm{CDCl}_{3}\right) \delta: 170.4,161.5\left(\mathrm{~d},{ }^{1} J_{\mathrm{CF}}=244.6 \mathrm{~Hz}\right), 137.8\left(\mathrm{~d},{ }^{3} J_{\mathrm{CF}}=6.4 \mathrm{~Hz}\right), 132.4$ $\left(\mathrm{d},{ }^{3} J_{\mathrm{CF}}=7.7 \mathrm{~Hz}\right), 132.0\left(\mathrm{~d},{ }^{4} J_{\mathrm{CF}}=3.2 \mathrm{~Hz}\right), 115.3\left(\mathrm{~d},{ }^{2} J_{\mathrm{CF}}=20.8 \mathrm{~Hz}\right), 114.0\left(\mathrm{~d},{ }^{2} J_{\mathrm{CF}}=22.4 \mathrm{~Hz}\right)$, 71.6, $56.3\left(\mathrm{~d},{ }^{4} J_{\mathrm{CF}}=1.3 \mathrm{~Hz}\right), 42.2,21.3,18.9,15.5 .{ }^{19} \mathrm{~F}\left(376 \mathrm{MHz}, \mathrm{CDCl}_{3}\right) \delta:-68.0,-70.3$. 


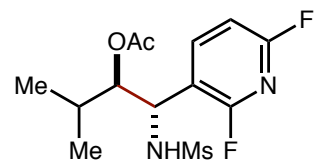

1-(2,6-difluoropyridin-3-yl)-3-methyl-1-(methylsulfonamido)butan-2-yl acetate (29): Isobutyraldehyde (55 $\mu \mathrm{L}, 0.6 \mathrm{mmol}$ ) was subjected to GP3 using $10 \% \mathrm{Mn}_{2}(\mathrm{CO})_{10}$ and upon column chromatography (40-50\% ethyl acetate/hexanes) provided $73 \% \mathbf{2 9}(1.2: 1$ d.r.) as an orange oil. $\mathrm{R}_{\mathrm{f}}: 0.27,0.22$ (40\% ethyl acetate/hexanes). HRMS (ESI-TOF) $\mathrm{m} / \mathrm{z}$ : calc'd for $[\mathrm{M}+\mathrm{Na}]^{+}$ 359.0853, found 359.0828.

Major diastereomer: ${ }^{1} \mathrm{H} \mathrm{NMR}\left(400 \mathrm{MHz}, \mathrm{CDCl}_{3}\right) \delta: 7.91-7.97(\mathrm{~m}, 1 \mathrm{H}), 6.86(\mathrm{dd}, J=2.9,8.2$ $\mathrm{Hz}, 1 \mathrm{H}), 5.47$ (d, $J=6.4 \mathrm{~Hz}, 1 \mathrm{H}), 5.02$ (t, $J=6.1 \mathrm{~Hz}, 1 \mathrm{H}), 4.95$ (dd, $J=6.6,6.6 \mathrm{~Hz}, 1 \mathrm{H}), 2.80(\mathrm{~s}$, $3 \mathrm{H}), 1.97(\mathrm{~s}, 3 \mathrm{H}), 1.88(\mathrm{~m}, J=6.7 \mathrm{~Hz}, 1 \mathrm{H}), 1.02(\mathrm{~d}, J=6.8 \mathrm{~Hz}, 3 \mathrm{H}), 0.94(\mathrm{~d}, J=6.8 \mathrm{~Hz}, 3 \mathrm{H})$. ${ }^{13} \mathrm{C}$ NMR $\left(100 \mathrm{MHz}, \mathrm{CDCl}_{3}\right) \delta: 170.8,161.3\left(\mathrm{dd},{ }^{1,3} J_{\mathrm{CF}}=234.5,14.3 \mathrm{~Hz}\right), 158.8\left(\mathrm{dd},{ }^{1,3} J_{\mathrm{CF}}=\right.$ $231.6,14.4 \mathrm{~Hz}), 145.0\left(\mathrm{dd},{ }^{3,3} J_{\mathrm{CF}}=4.5,7.8 \mathrm{~Hz}\right), 116.9\left(\mathrm{dd},{ }^{2,4} J_{\mathrm{CF}}=25.8,6.0 \mathrm{~Hz}\right), 106.8\left(\mathrm{dd},{ }^{2,4} J_{\mathrm{CF}}\right.$ $=34.4,5.6 \mathrm{~Hz}), 79.4,52.2\left(\mathrm{~d},{ }^{4} J_{\mathrm{CF}}=2.5 \mathrm{~Hz}\right), 42.1,29.2,20.7,19.5,17.0 .{ }^{19} \mathrm{~F}\left(376 \mathrm{MHz}, \mathrm{CDCl}_{3}\right)$ $\delta:-67.6,-70.2$.

Minor diastereomer: ${ }^{1} \mathrm{H} \mathrm{NMR}\left(400 \mathrm{MHz}, \mathrm{CDCl}_{3}\right) \delta: 7.84(\mathrm{~m}, 1 \mathrm{H}), 6.86(\mathrm{dd}, J=8.1,2.9 \mathrm{~Hz}, 1 \mathrm{H})$, $5.25(\mathrm{~d}, \mathrm{~J}=7.6 \mathrm{~Hz}, 1 \mathrm{H}), 4.96(\mathrm{~m}, 2 \mathrm{H}), 2.82(\mathrm{~s}, 3 \mathrm{H}), 2.06(\mathrm{~s}, 3 \mathrm{H}), 1.89(\mathrm{~m}, J=6.7 \mathrm{~Hz}, 1 \mathrm{H}), 1.05$ $(\mathrm{d}, J=6.8 \mathrm{~Hz}, 3 \mathrm{H}), 0.93(\mathrm{~d}, J=6.7 \mathrm{~Hz}, 3 \mathrm{H}) .{ }^{13} \mathrm{C}$ NMR $\left(100 \mathrm{MHz}, \mathrm{CDCl}_{3}\right) \delta: 170.6,161.2(\mathrm{dd}$, $\left.{ }^{1,3} J_{\mathrm{CF}}=248.8,14.5 \mathrm{~Hz}\right), 158.4\left(\mathrm{dd},{ }^{1,3} J_{\mathrm{CF}}=246.4,14.3 \mathrm{~Hz}\right), 143.4\left(\mathrm{dd},{ }^{3,3} J_{\mathrm{CF}}=7.8,4.7 \mathrm{~Hz}\right), 118.3$ $\left(\mathrm{dd},{ }^{2,4} J_{\mathrm{CF}}=25.7,6.0 \mathrm{~Hz}\right), 106.9\left(\mathrm{dd},{ }^{2,4} J_{\mathrm{CF}}=34.5,5.7 \mathrm{~Hz}\right), 79.0,53.3\left(\mathrm{~d},{ }^{4} J_{\mathrm{CF}}=3.0 \mathrm{~Hz}\right), 42.3$, 29.3, 20.7, 19.2, 17.4. ${ }^{19} \mathrm{~F}\left(376 \mathrm{MHz}, \mathrm{CDCl}_{3}\right) \delta:-67.9,-70.1$.

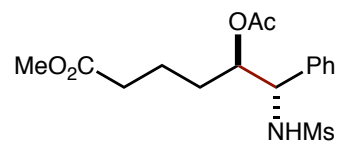

methyl 5-acetoxy-6-(methylsulfonamido)-6-phenylhexanoate (30): Aldehyde S30 (75 mg, 0.6 $\mathrm{mmol}$ ) was subjected to GP3 using $10 \% \mathrm{Mn}_{2}(\mathrm{CO})_{10}$ catalyst and upon column chromatography (20 $-40 \%$ ethyl acetate/hexanes) provided $70 \% 30$ (3:1 d.r.). Rf: 0.39 (50\% ethyl acetate/hexanes). IR (film): 3285, 2980, 1734, 1321, 1235, 1153. HRMS (ESI-TOF) m/z: calc'd for [M+Na] 380.1144 , found 380.1155 .

Major diastereomer: ${ }^{1} \mathrm{H}$ NMR (400 $\left.\mathrm{MHz}, \mathrm{CDCl}_{3}\right) \delta: 7.28-7.40(\mathrm{~m}, 5 \mathrm{H}), 5.42(\mathrm{~d}, J=8.3 \mathrm{~Hz}$, $1 \mathrm{H}), 5.11-5.16(\mathrm{~m}, 1 \mathrm{H}), 4.68(\mathrm{dd}, J=6.2,8.7 \mathrm{~Hz}, 1 \mathrm{H}), 3.64(\mathrm{~s}, 3 \mathrm{H}), 2.63(\mathrm{~s}, 3 \mathrm{H}), 2.27(\mathrm{t}, J=6.9$ $\mathrm{Hz}), 2.02$ (s, 3H), $1.53-1.68(\mathrm{~m}, 4 \mathrm{H}) .{ }^{13} \mathrm{C} \mathrm{NMR}\left(100 \mathrm{MHz}, \mathrm{CDCl}_{3}\right) \delta: 173.0,171.0,137.1,129.0$, $128.6,127.7,75.5,60.2,51.7,42.1,33.5,29.8,21.1,20.9$.

Minor diastereomer: ${ }^{1} \mathrm{H}$ NMR $\left(400 \mathrm{MHz}, \mathrm{CDCl}_{3}\right) \delta: 7.28-7.40(\mathrm{~m}, 5 \mathrm{H}), 5.31(\mathrm{~d}, J=87.8 \mathrm{~Hz}$, $1 \mathrm{H}), 5.11-5.16(\mathrm{~m}, 1 \mathrm{H}), 4.55(\mathrm{dd}, J=6.2,8.7 \mathrm{~Hz}, 1 \mathrm{H}), 3.63(\mathrm{~s}, 3 \mathrm{H}), 2.57(\mathrm{~s}, 3 \mathrm{H}), 2.27(\mathrm{t}, J=6.9$ $\mathrm{Hz}), 2.08$ (s, 3H), 1.53 - $1.68(\mathrm{~m}, 4 \mathrm{H}) .{ }^{13} \mathrm{C} \mathrm{NMR}\left(100 \mathrm{MHz}, \mathrm{CDCl}_{3}\right) \delta: 173.5,171.1,138.4,129.3$, 128.8, 127.1, 75.4, 60.8, 51.7, 42.2, 33.6, 31.0, 21.2, 20.7. 
<smiles>CCCCCCC(OC(C)=O)C(N=[N+]([O-])c1ccccc1)c1ccccc1</smiles>

7-cyano-1-(methylsulfonamido)-1-phenylheptan-2-yl acetate (31): Aldehyde S31 (75 mg, 0.6 mmol) was subjected to GP3 using $10 \% \mathrm{Mn}_{2}(\mathrm{CO})_{10}$ catalyst and upon column chromatography (20 $-40 \%$ ethyl acetate/hexanes) provided $70 \% 31$ (3:1 d.r.). Rf: 0.33 (50\% ethyl acetate/hexanes). IR (film): 3281, 2980, 2970, 2889, 1735, 1376, 1236, 1153. HRMS (ESI-TOF) m/z: calc'd for $[\mathrm{M}+\mathrm{Na}]^{+}$375.1354, found 375.1361 .

Major diastereomer: ${ }^{1} \mathrm{H}$ NMR $\left(400 \mathrm{MHz}, \mathrm{CDCl}_{3}\right) \delta: 7.27-7.41(\mathrm{~m}, 5 \mathrm{H}), 5.26(\mathrm{~d}, J=8.1 \mathrm{~Hz}$, $1 \mathrm{H}), 5.09-5.15(\mathrm{~m}, 1 \mathrm{H}), 4.67(\mathrm{dd}, J=4.7,8.3 \mathrm{~Hz}, 1 \mathrm{H}), 2.63(\mathrm{~s}, 3 \mathrm{H}), 2.30(\mathrm{t}, J=7.1 \mathrm{~Hz}, 2 \mathrm{H}), 2.03$ $(\mathrm{s}, 3 \mathrm{H}), 1.55-1.64(\mathrm{~m}, 4 \mathrm{H}), 1.34-1.43(\mathrm{~m}, 4 \mathrm{H}) .{ }^{13} \mathrm{C} \mathrm{NMR}\left(100 \mathrm{MHz}, \mathrm{CDCl}_{3}\right) \delta: 170.9,137.2$, $128.9,128.5,127.7,119.7,75.7,60.1,42.0,30.2,28.3,25.2,24.7,21.1,17.1$

Minor diastereomer: ${ }^{1} \mathrm{H}$ NMR $\left(400 \mathrm{MHz} \mathrm{CDCl}_{3}\right) \delta: 7.27-7.41(\mathrm{~m}, 5 \mathrm{H}), 5.21(\mathrm{~d}, J=8.8 \mathrm{~Hz}$, $1 \mathrm{H}), 5.05-5.09(\mathrm{~m}, 1 \mathrm{H}), 4.55(\mathrm{dd}, J=6.0,8.8 \mathrm{~Hz}, 1 \mathrm{H}), 2.57(\mathrm{~s}, 3 \mathrm{H}), 2.30(\mathrm{t}, J=7.0 \mathrm{~Hz}, 2 \mathrm{H}), 2.07$ $(\mathrm{s}, 3 \mathrm{H}), 1.55-1.64(\mathrm{~m}, 4 \mathrm{H}), 1.34-1.43(\mathrm{~m}, 4 \mathrm{H}) .{ }^{13} \mathrm{C} \mathrm{NMR}\left(100 \mathrm{MHz}, \mathrm{CDCl}_{3}\right) \delta: 171.0,138.4$, $129.3,128.7,127.0,119.7,75.7,60.7,42.0,31.2,28.3,25.2,24.5,21.1,17.1$.<smiles>COC(CCCCCCl)[C@H](N)c1ccccc1</smiles>

7-chloro-1-(methylsulfonamido)-1-phenylheptan-2-yl acetate (32): Aldehyde S32 (80.8 mg, $0.6 \mathrm{mmol})$ was subjected to GP3 and upon column chromatography $(15-25 \%$ ethyl acetate/hexanes) provided $74 \% 32$ (3:1 d.r.). $\mathrm{R}_{\mathrm{f}}$ : 0.26 (30\% ethyl acetate/hexanes). IR (film): 3282 , 2980, 2889, 1734, 1320, 1236, 906. HRMS (ESI-TOF) m/z: calc'd for $[\mathrm{M}+\mathrm{Na}]^{+} 384.1012$, found 384.1011 .

Major diastereomer: ${ }^{1} \mathrm{H} \mathrm{NMR}\left(400 \mathrm{MHz}, \mathrm{CDCl}_{3}\right) \delta: 7.28-7.40(\mathrm{~m}, 5 \mathrm{H}), 5.27(\mathrm{~d}, J=7.9 \mathrm{~Hz}$, $1 \mathrm{H}), 5.12-5.15(\mathrm{~m}, 1 \mathrm{H}), 4.68(\mathrm{dd}, J=4.3,7.8 \mathrm{~Hz}, 1 \mathrm{H}), 3.47-3.50(\mathrm{~m}, 2 \mathrm{H}), 2.63(\mathrm{~s}, 3 \mathrm{H}), 2.03$ $(\mathrm{s}, 3 \mathrm{H}), 1.68-1.73(\mathrm{~m}, 2 \mathrm{H}), 1.48-1.52(\mathrm{~m}, 2 \mathrm{H}), 1.33-1.41(\mathrm{~m}, 3 \mathrm{H}), 1.25-1.28(\mathrm{~m}, 1 \mathrm{H}) .{ }^{13} \mathrm{C}$ NMR $\left(100 \mathrm{MHz}, \mathrm{CDCl}_{3}\right) \delta: 171.0,137.2,128.9,128.5,127.8,75.9,60.1,44.9,42.0,32.4,30.3$, 26.6, 24.8, 21.1.

Minor diastereomer: ${ }^{1} \mathrm{H}$ NMR $\left(400 \mathrm{MHz}, \mathrm{CDCl}_{3}\right) \delta: 7.28-7.40(\mathrm{~m}, 5 \mathrm{H}), 5.22(\mathrm{~d}, J=8.8 \mathrm{~Hz}$, $1 \mathrm{H}), 5.07-5.10(\mathrm{~m}, 1 \mathrm{H}), 4.55(\mathrm{dd}, J=6.1,8.9 \mathrm{~Hz}, 1 \mathrm{H}), 3.47-3.50(\mathrm{~m}, 2 \mathrm{H}), 2.657(\mathrm{~s}, 3 \mathrm{H}), 2.07$ $(\mathrm{s}, 3 \mathrm{H}), 1.68-1.73(\mathrm{~m}, 2 \mathrm{H}), 1.48-1.52(\mathrm{~m}, 2 \mathrm{H}), 1.33-1.41(\mathrm{~m}, 3 \mathrm{H}), 1.25-1.28(\mathrm{~m}, 1 \mathrm{H}) .{ }^{13} \mathrm{C}$ NMR $\left(100 \mathrm{MHz}, \mathrm{CDCl}_{3}\right) \delta: 171.1,138.5,129.3,128.7,127.1,75.8,60.8,44.9,42.1,32.4,31.4$, $26.5,24.6,21.1$. 


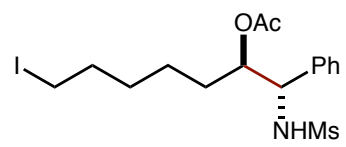

7-iodo-1-(methylsulfonamido)-1-phenylheptan-2-yl acetate (33): Aldehyde S33 (119 mg, 0.6 mmol) was subjected to GP3 using $10 \% \mathrm{Mn}_{2}(\mathrm{CO})_{10}$ and ${ }^{i} \operatorname{Pr}_{2} \mathrm{NEt}(171 \mu \mathrm{L}, 1 \mathrm{mmol})$ and upon column chromatography ( $15-25 \%$ ethyl acetate/hexanes) provided $54 \% 33$ (3:1 d.r.). $\mathrm{R}_{\mathrm{f}}: 0.26$

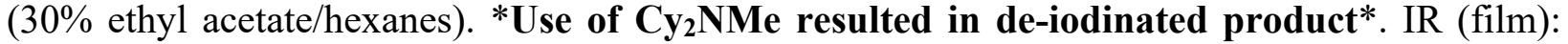
3280, 2980, 2889, 1735, 1372, 1234, 1154, 1043. HRMS (ESI-TOF) m/z: calc'd for [M+Na] ${ }^{+}$ 384.1012, found 384.1011.

Major diastereomer: ${ }^{1} \mathrm{H}$ NMR $\left(400 \mathrm{MHz}, \mathrm{CDCl}_{3}\right) \delta: 7.28-7.39(\mathrm{~m}, 5 \mathrm{H}), 5.23(\mathrm{~d}, J=8.2 \mathrm{~Hz}$, $1 \mathrm{H}), 5.11-5.15(\mathrm{~m}, 1 \mathrm{H}), 4.68(\mathrm{dd}, J=4.5,8.1 \mathrm{~Hz}, 1 \mathrm{H}), 3.13(\mathrm{t}, J=6.9 \mathrm{~Hz}, 2 \mathrm{H}), 2.63(\mathrm{~s}, 3 \mathrm{H})$, $2.03(\mathrm{~s}, 3 \mathrm{H}), 1.72-1.79(\mathrm{~m}, 2 \mathrm{H}), 1.48-1.52(\mathrm{~m}, 2 \mathrm{H}), 1.29-1.37(\mathrm{~m}, 4 \mathrm{H}), 1.25-1.28(\mathrm{~m}, 1 \mathrm{H})$. ${ }^{13} \mathrm{C}$ NMR $\left(100 \mathrm{MHz}, \mathrm{CDCl}_{3}\right) \delta: 171.0,137.1,129.0,128.6,127.8,76.0,60.2,42.1,33.2,30.3$, $30.2,24.5,21.2,6.8$.

Minor diastereomer: ${ }^{1} \mathrm{H}$ NMR $\left(400 \mathrm{MHz}, \mathrm{CDCl}_{3}\right) \delta: 7.28-7.39(\mathrm{~m}, 5 \mathrm{H}), 5.22(\mathrm{~d}, J=8.8 \mathrm{~Hz}$, $1 \mathrm{H}), 5.07-5.10(\mathrm{~m}, 1 \mathrm{H}), 4.55(\mathrm{dd}, J=6.1,8.9 \mathrm{~Hz}, 1 \mathrm{H}), 3.13(\mathrm{t}, J=6.9 \mathrm{~Hz}, 2 \mathrm{H}), 2.57(\mathrm{~s}, 3 \mathrm{H})$, $2.07(\mathrm{~s}, 3 \mathrm{H}), 1.72-1.79(\mathrm{~m}, 2 \mathrm{H}), 1.48-1.52(\mathrm{~m}, 2 \mathrm{H}), 1.29-1.37(\mathrm{~m}, 4 \mathrm{H}), 1.25-1.28(\mathrm{~m}, 1 \mathrm{H})$. ${ }^{13} \mathrm{C}$ NMR $\left(100 \mathrm{MHz}, \mathrm{CDCl}_{3}\right) \delta: 171.1,138.6,129.3,128.7,127.1,75.8,60.7,42.2,31.4,30.2$, $30.2,24.2,21.1,6.8$.

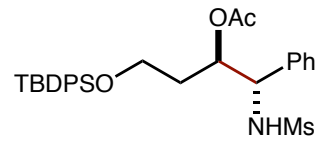

4-((tert-butyldiphenylsilyl)oxy)-1-(methylsulfonamido)-1-phenylbutan-2-yl acetate (34): Aldehyde S34 (93.7 mg, $0.3 \mathrm{mmol}$ ) was subjected to GP4 and upon column chromatography (15 $-25 \%$ ethyl acetate/hexanes) provided $68 \% 34$ (3:1 d.r.). Rf: 0.36 (30\% ethyl acetate/hexanes). IR (film): 3281, 2980, 2970, 2931, 2888, 1736, 1154, 907. HRMS (ESI-TOF) m/z: calc'd for $[\mathrm{M}+\mathrm{Na}]^{+}$562.2059, found 562.2068.

Major diastereomer: ${ }^{1} \mathrm{H}$ NMR $\left(400 \mathrm{MHz}, \mathrm{CDCl}_{3}\right) \delta: 7.56-7.60(\mathrm{~m}, 4 \mathrm{H}), 7.28-7.44(\mathrm{~m}, 11 \mathrm{H})$, $5.50(\mathrm{~d}, J=7.9 \mathrm{~Hz}, 1 \mathrm{H}), 5.39(\mathrm{td}, J=3.9,8.8 \mathrm{~Hz}, 1 \mathrm{H}), 4.83(\mathrm{dd}, J=4.1,7.9 \mathrm{~Hz}, 1 \mathrm{H}), 3.57-3.70$ $(\mathrm{m}, 2 \mathrm{H}), 2.67(\mathrm{~s}, 3 \mathrm{H}), 1.99,(\mathrm{~s}, 3 \mathrm{H}), 1.71-1.83(\mathrm{~m}, 1 \mathrm{H}) 1.57-1.66(\mathrm{~m}, 1 \mathrm{H}), 1.02(\mathrm{~s}, 9 \mathrm{H}) .{ }^{13} \mathrm{C}$ NMR $\left(100 \mathrm{MHz}, \mathrm{CDCl}_{3}\right) \delta: 171.2,137.2,135.7,133.4,129.9,128.9,128.4,127.9,127.6,73.2$, $60.0,59.7,42.1,32.6,27.0,21.1,19.3$.

Minor diastereomer: ${ }^{1} \mathrm{H}$ NMR $\left(400 \mathrm{MHz}, \mathrm{CDCl}_{3}\right) \delta: 7.56-7.60(\mathrm{~m}, 4 \mathrm{H}), 7.28-7.44(\mathrm{~m}, 11 \mathrm{H})$, $5.40-5.46(\mathrm{~m}, 1 \mathrm{H}), 5.32(\mathrm{~d}, J=8.4 \mathrm{~Hz}, 1 \mathrm{H}), 4.58(\mathrm{dd}, J=7.0,8.6 \mathrm{~Hz}, 1 \mathrm{H}) 3.57-3.70(\mathrm{~m}, 2 \mathrm{H})$, $2.55(\mathrm{~s}, 3 \mathrm{H}), 2.02,(\mathrm{~s}, 3 \mathrm{H}), 1.71-1.83(\mathrm{~m}, 1 \mathrm{H}) 1.57-1.66(\mathrm{~m}, 1 \mathrm{H}), 1.02(\mathrm{~s}, 9 \mathrm{H}) .{ }^{13} \mathrm{C}$ NMR $(100$ $\left.\mathrm{MHz}, \mathrm{CDCl}_{3}\right) \delta: 171.0,138.6,135.6,133.5,129.8,129.3,128.7,127.8,127.3,73.0,61.2,59.7$, 42.1, 34.3, 26.9, 21.1, 19.2. 
$\overbrace{\text { i }}^{\mathrm{N} H \mathrm{H} M \mathrm{~s}}$

4-(1,3-dioxoisoindolin-2-yl)-1-(methylsulfonamido)-1-phenylbutan-2-yl acetate (35): Aldehyde S35 (61.0 mg, $0.3 \mathrm{mmol})$ was subjected to GP3 and upon column chromatography (20 $-50 \%$ ethyl acetate/hexanes) provided $50 \% 35$ (3:1 d.r.) as an off-white solid. $R_{\mathrm{f}}: 0.28$ (50\% ethyl acetate/hexanes). IR (film): 2984, 2970, 1736, 1372, 1233, 1043. HRMS (ESI-TOF) m/z: calc'd for $[\mathrm{M}+\mathrm{Na}]^{+} 453.1096$, found 453.1121 .

Major diastereomer: ${ }^{1} \mathrm{H}$ NMR $\left(400 \mathrm{MHz}, \mathrm{CDCl}_{3}\right) \delta: 7.81-7.84(\mathrm{~m}, 2 \mathrm{H}), 7.69-7.72(\mathrm{~m}, 2 \mathrm{H})$, $7.29-7.37(\mathrm{~m}, 5 \mathrm{H}) 5.65(\mathrm{~d}, J=8.1 \mathrm{~Hz}, 1 \mathrm{H}), 5.08-5.13(\mathrm{~m}, 1 \mathrm{H}), 4.70-4.75(\mathrm{~m}, 1 \mathrm{H}), 3.69-3.79$ $(\mathrm{m}, 2 \mathrm{H}), 2.63(\mathrm{~s}, 3 \mathrm{H}), 2.00(\mathrm{~s}, 3 \mathrm{H}), 1.98-2.05(\mathrm{~m}, 1 \mathrm{H}), 1.82-1.90(\mathrm{~m}, 1 \mathrm{H}) .{ }^{13} \mathrm{C} \mathrm{NMR}(100 \mathrm{MHz}$, $\left.\mathrm{CDCl}_{3}\right) \delta: 171.1,168.4,137.0,134.2,132.1,129.0,128.6,127.6,123.4,73.5,60.3,42.0,34.6$, 29.6, 21.0.

Minor diastereomer: ${ }^{1} \mathrm{H} \mathrm{NMR}\left(400 \mathrm{MHz}, \mathrm{CDCl}_{3}\right) \delta: 7.81-7.84(\mathrm{~m}, 2 \mathrm{H}), 7.69-7.72(\mathrm{~m}, 2 \mathrm{H})$, $7.29-7.37(\mathrm{~m}, 5 \mathrm{H}) 5.36(\mathrm{~d}, J=9.1 \mathrm{~Hz}, 1 \mathrm{H}), 5.08-5.13(\mathrm{~m}, 1 \mathrm{H}), 4.70-4.75(\mathrm{~m}, 1 \mathrm{H}), 3.69-3.79$ $(\mathrm{m}, 2 \mathrm{H}), 2.59(\mathrm{~s}, 3 \mathrm{H}), 2.04(\mathrm{~s}, 3 \mathrm{H}), 1.98-2.05(\mathrm{~m}, 1 \mathrm{H}), 1.82-1.90(\mathrm{~m}, 1 \mathrm{H}) .{ }^{13} \mathrm{C} \mathrm{NMR}(100 \mathrm{MHz}$, $\left.\mathrm{CDCl}_{3}\right) \delta: 170.9,168.3,138.1,134.1,132.1,129.3,128.7,127.1,123.4,73.6,60.3,42.0,34.3$, $30.4,21.0$.<smiles>COC(CCCCCNC(=O)C(F)(F)F)C(N)c1ccccc1</smiles>

1-(methylsulfonamido)-1-phenyl-7-(2,2,2-trifluoroacetamido)heptan-2-yl acetate (36): Aldehyde S36 (109.9 mg, $0.6 \mathrm{mmol}$ ) was subjected to GP3 and upon column chromatography (30 - 50\% ethyl acetate/hexanes) provided $65 \% 36$ (3:1 d.r.) as a slightly yellow oil. $\mathrm{R}_{\mathrm{f}}: 0.31(50 \%$ ethyl acetate/hexanes). IR (film): 3326, 3103, 2937, 2859, 1708, 1208, 1183, 1152. HRMS (ESITOF) $\mathrm{m} / \mathrm{z}$ : calc'd for $[\mathrm{M}+\mathrm{H}]^{+}$461.1334, found 461.1343 .

Major diastereomer: ${ }^{1} \mathrm{H}$ NMR $\left(400 \mathrm{MHz} \mathrm{CDCl}_{3}\right) \delta: 7.29-7.43(\mathrm{~m}, 5 \mathrm{H}), 6.26$ (brs, 1H), 5.61 $(\mathrm{d}, J=8.8 \mathrm{~Hz}, 1 \mathrm{H}), 5.14-5.18(\mathrm{~m}, 1 \mathrm{H}), 4.64(\mathrm{dd}, J=4.9,8.8 \mathrm{~Hz}, 1 \mathrm{H}), 3.28-3.40(\mathrm{~m}, 2 \mathrm{H}), 2.61$ $(\mathrm{s}, 3 \mathrm{H}), 2.00,(\mathrm{~s}, 3 \mathrm{H}), 1.49-1.60(\mathrm{~m}, 4 \mathrm{H}) 1.25-1.37(\mathrm{~m}, 4 \mathrm{H}) .{ }^{13} \mathrm{C}$ NMR $\left(100 \mathrm{MHz}, \mathrm{CDCl}_{3}\right) \delta$ : $171.0,157.4\left(\mathrm{q},{ }^{2} J_{\mathrm{CF}}=36.7 \mathrm{~Hz}\right), 137.2,129.0,128.6,127.7,116.0\left(\mathrm{q},{ }^{1} J_{\mathrm{CF}}=287.9 \mathrm{~Hz}\right), 75.7,60.2$, 42.0, 39.7, 30.3, 28.6, 26.1, 24.7, 21.1. ${ }^{19} \mathrm{~F}\left(376 \mathrm{MHz}, \mathrm{CDCl}_{3}\right) \delta$ : - 75.9.

Minor diastereomer: ${ }^{1} \mathrm{H}$ NMR $\left(400 \mathrm{MHz}, \mathrm{CDCl}_{3}\right) \delta: 7.29-7.43(\mathrm{~m}, 5 \mathrm{H}), 6.26$ (brs, $\left.1 \mathrm{H}\right), 5.44$ $(\mathrm{d}, J=10.1 \mathrm{~Hz}, 1 \mathrm{H}), 5.08-5.10(\mathrm{~m}, 1 \mathrm{H}), 4.55(\mathrm{dd}, J=5.6,9.2 \mathrm{~Hz}, 1 \mathrm{H}), 3.28-3.40(\mathrm{~m}, 2 \mathrm{H})$, $2.58(\mathrm{~s}, 3 \mathrm{H}), 2.05,(\mathrm{~s}, 3 \mathrm{H}), 1.49-1.60(\mathrm{~m}, 4 \mathrm{H}) 1.25-1.37(\mathrm{~m}, 4 \mathrm{H}) .{ }^{13} \mathrm{C} \mathrm{NMR}\left(100 \mathrm{MHz}, \mathrm{CDCl}_{3}\right)$ $\delta: 171.0,157.4\left(\mathrm{q},{ }^{2} J_{\mathrm{CF}}=36.7 \mathrm{~Hz}\right), 138.5,129.3,128.7,127.0,116.0\left(\mathrm{q},{ }^{1} J_{\mathrm{CF}}=287.9 \mathrm{~Hz}\right), 75.9$, $60.5,42.1,39.7,31.3,28.6,26.1,24.5,21.0 .{ }^{19} \mathrm{~F}\left(376 \mathrm{MHz}, \mathrm{CDCl}_{3}\right) \delta:-75.9$. 
<smiles>CCCCC(N)C(CCCC)OC(C)=O</smiles>

6-((4-methylphenyl)sulfonamido)decan-5-yl acetate (37): To the $\alpha$ oxy iodide reaction described in GP3, Imine $\mathbf{S 2 7}$ (47.8 mg, $0.2 \mathrm{mmol}$ ), Zn (26.2 mg, $0.4 \mathrm{mmol}$ ), Hantzsch ester (37.9 $\mathrm{mg}, 0.15 \mathrm{mmol})$, and $\mathrm{Mn}_{2}(\mathrm{CO})_{10}(15.6 \mathrm{mg}, 0.04 \mathrm{mmol})$ were added at the same time. The reaction was degassed by stirring at $-78^{\circ} \mathrm{C}$ for $20 \mathrm{~min}$ under vacuum. The reaction was irradiated with Blue LEDs (34W). Upon column chromatography (20-40\% ethyl acetate/hexanes) provides 54\% 37 (1:1 d.r.) as a yellow oil ( ${ }^{1} \mathrm{H}$ NMR yield). $\mathrm{R}_{\mathrm{f}}$ : 0.5 (30\% ethyl acetate/hexanes). HRMS (ESI-TOF) $\mathrm{m} / \mathrm{z}$ : calc'd for $[\mathrm{M}+\mathrm{H}]^{+} 370.2052$, found 370.2043 .

Major diastereomer: ${ }^{1} \mathrm{H}$ NMR $\left(400 \mathrm{MHz}, \mathrm{CDCl}_{3}\right) \delta: 7.75(\mathrm{t}, J=7.9 \mathrm{~Hz}, 1 \mathrm{H}), 7.29(\mathrm{~d}, J=7.9 \mathrm{~Hz}$, $1 \mathrm{H}), 4.83-4.86(\mathrm{~m}, 1 \mathrm{H}), 4.64(\mathrm{~d}, J=9.5 \mathrm{~Hz}, 1 \mathrm{H}), 3.31-3.40(\mathrm{~m}, 1 \mathrm{H}), 2.41(\mathrm{~s}, 3 \mathrm{H}), 1.99(\mathrm{~s}, 3 \mathrm{H})$, 1.04-1.51 (m, 12H), 0.73-0.86 (m, 6H). ${ }^{13} \mathrm{C} \mathrm{NMR}\left(150 \mathrm{MHz}, \mathrm{CDCl}_{3}\right)$ \&: 170.6, 138.7, 129.7, 127.1, 74.4, 56.1, 31.1, 29.7, 27.7, 27.5, 22.5, 22.4, 21.0, 13.9, 13.9.

Minor diastereomer: ${ }^{1} \mathrm{H}$ NMR $\left(400 \mathrm{MHz}, \mathrm{CDCl}_{3}\right) \delta: 7.75(\mathrm{t}, J=7.9 \mathrm{~Hz}, 1 \mathrm{H}), 7.29(\mathrm{~d}, J=7.9 \mathrm{~Hz}$, $1 \mathrm{H}), 4.49-4.54(\mathrm{~m}, 1 \mathrm{H}), 4.78(\mathrm{~d}, J=9.2 \mathrm{~Hz}, 1 \mathrm{H}), 3.31-3.40(\mathrm{~m}, 1 \mathrm{H}), 2.41(\mathrm{~s}, 3 \mathrm{H}), 1.99(\mathrm{~s}, 3 \mathrm{H})$, $1.04-1.51(\mathrm{~m}, 12 \mathrm{H}), 0.73-0.86(\mathrm{~m}, 6 \mathrm{H}) .{ }^{13} \mathrm{C} \mathrm{NMR}\left(150 \mathrm{MHz}, \mathrm{CDCl}_{3}\right) \delta: 171.3,138.4,129.7,127.2$, $76.1,56.2,32.8,30.4,27.8,27.7,22.4,22.3,21.6,21.0,13.9,13.8$.

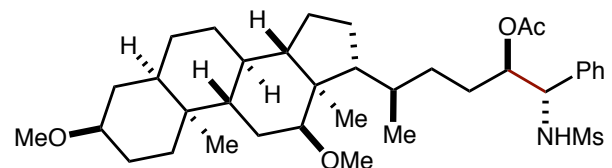

(5R)-5-((3R,5R,8R,9S,10S,12S,13R,14S,17R)-3,12-dimethoxy-10,13dimethylhexadecahydro-1H-cyclopenta $[a] 38$ henanthrene-17-yl)-1-(methylsulfonamido)-1phenylhexan-2-yl acetate (38): Aldehyde $\mathbf{S 3 7}(121 \mathrm{mg}, 0.3 \mathrm{mmol})$ was subjected to GP4 using $15 \% \mathrm{Mn}_{2}(\mathrm{CO})_{10}$ and Hantzsch Ester $(25.3 \mathrm{mg}, 0.1 \mathrm{mmol})$ and upon column chromatography (15 $-25 \%$ ethyl acetate/hexanes) provided 54\% 38 (3:3:1:1 d.r.). $R_{f}: 0.29$ (30\% ethyl acetate/hexanes). IR (film): 2985, 2941, 1736, 1372, 1233, 1043. HRMS (ESI-TOF) m/z: calc'd for [M+Na] ${ }^{+}$ 654.3804, found 654.3784.

Major diastereomers (mixture): ${ }^{1} \mathrm{H}$ NMR $\left(400 \mathrm{MHz}, \mathrm{CDCl}_{3}\right) \delta: 7.28-7.39(\mathrm{~m}, 5 \mathrm{H}), 5.21-5.22$ $(\mathrm{m}, 1 \mathrm{H}), 5.06-5.19(\mathrm{~m}, 1 \mathrm{H}), 4.68-4.71(\mathrm{~m}, 1 \mathrm{H}), 3.33(\mathrm{~s}, 3 \mathrm{H}), 3.30-3.37(\mathrm{~m}, 1 \mathrm{H}), 3.22(\mathrm{~m}, 2 \mathrm{H})$, $3.11-3.17(\mathrm{~m}, 1 \mathrm{H}), 2.63(3,3 \mathrm{H}), 2.03(\mathrm{~s}, 3 \mathrm{H}), 1.67-1.82(\mathrm{~m}, 8 \mathrm{H}), 1.50-1.58(\mathrm{~m}, 2 \mathrm{H}), 0.91(\mathrm{~m}$, $16 \mathrm{H}), 0.90(\mathrm{~s}, 3 \mathrm{H}), 0.79-0.82(\mathrm{~m}, 3 \mathrm{H}), 0.62(\mathrm{~s}, 3 \mathrm{H}) .{ }^{13} \mathrm{C} \mathrm{NMR}\left(100 \mathrm{MHz}, \mathrm{CDCl}_{3}\right) \delta: 171.1,171.0$, 137.3, 137.2, 128.8, 128.5, 128.4, 128.0, 127.8, 80.6, 76.4, 60.3, 59.8, 55.6, 48.9, 46.7, 46.4, 42.2, $42.1,36.1,35.4,34.6,33.6,32.7,31.5,31.4,27.4,26.9,26.2,23.7,23.4,22.0,21.1,17.8,17.6$, 12.8 .

Minor diastereomers (mixture): ${ }^{1} \mathrm{H}$ NMR $\left(400 \mathrm{MHz}, \mathrm{CDCl}_{3}\right) \delta: 7.28-7.39(\mathrm{~m}, 5 \mathrm{H}), 5.13-5.17$ $(\mathrm{m}, 1 \mathrm{H}), 5.00-5.04(\mathrm{~m}, 1 \mathrm{H}), 4.53-4.58(\mathrm{~m}, 1 \mathrm{H}), 3.33(\mathrm{~s}, 3 \mathrm{H}), 3.30-3.37(\mathrm{~m}, 1 \mathrm{H}), 3.22(\mathrm{~m}, 2 \mathrm{H})$, $3.11-3.17(\mathrm{~m}, 1 \mathrm{H}), 2.56(3,3 \mathrm{H}), 2.08(\mathrm{~s}, 3 \mathrm{H}), 1.67-1.82(\mathrm{~m}, 8 \mathrm{H}), 1.50-1.58(\mathrm{~m}, 2 \mathrm{H}), 0.91(\mathrm{~m}$, $16 \mathrm{H}), 0.90(\mathrm{~s}, 3 \mathrm{H}), 0.79-0.82(\mathrm{~m}, 3 \mathrm{H}), 0.62(\mathrm{~s}, 3 \mathrm{H}) .{ }^{13} \mathrm{C} \mathrm{NMR}\left(100 \mathrm{MHz}, \mathrm{CDCl}_{3}\right) \delta: 171.2,170.9$, $138.8,138.7,129.2,128.6,128.5,127.2,127.1,82.3,76.1,60.9,60.3,55.7,48.9,46.6,46.4,42.1$, $42.1,35.5,35.5,35.1,31.2,31.0,29.8,29.1,27.5,27.5,27.3,23.8,23.4,21.2,21.1,17.8,17.5$, 12.8 . 


\section{Asymmetric aza-pinacol coupling}<smiles>O=S(/N=C/c1ccccc1)c1ccc(F)cc1F</smiles>

(R,E)- $\boldsymbol{N}$-benzylidene-2,4-difluorobenzenesulfinamide (S39): Synthesized by previously reported procedure. ${ }^{12}$ White solid. IR (film): 3660, 3360, 2970, 2890, 1600, 1460, 1380, 1320, 1160, 1130, 1100, 949, 816, 736. ${ }^{1} \mathrm{H}$ NMR (400 MHz, $\left.\mathrm{CDCl}_{3}\right) \delta: 8.76(\mathrm{~d}, J=1.3 \mathrm{~Hz}, 1 \mathrm{H}), 7.89-$ $7.83(\mathrm{~m}, 2 \mathrm{H}), 7.67(\mathrm{ddd}, J=8.8,7.5,6.2 \mathrm{~Hz}, 1 \mathrm{H}), 7.56-7.50(\mathrm{~m}, 1 \mathrm{H}), 7.50-7.43(\mathrm{~m}, 2 \mathrm{H}), 7.05$ - $6.98(\mathrm{~m}, 1 \mathrm{H}), 6.96-6.89(\mathrm{~m}, 1 \mathrm{H}) .{ }^{13} \mathrm{C} \mathrm{NMR}\left(151 \mathrm{MHz}, \mathrm{CDCl}_{3}\right) \delta: 165.3(\mathrm{dd}, J=254.6,11.1$ Hz), 161.9, 159.9 (dd, $J=254.3,12.6 \mathrm{~Hz}), 133.8,133.1,129.9,129.1,128.3(\mathrm{dd}, J=16.6,3.7 \mathrm{~Hz})$, $126.8(\mathrm{dd}, J=10.4,3.8 \mathrm{~Hz}), 112.6(\mathrm{dd}, J=22.1,3.5 \mathrm{~Hz}), 105.1(\mathrm{dd}, J=25.9,24.4 \mathrm{~Hz}) .{ }^{19} \mathrm{~F} \mathrm{NMR}$ (377 MHz, $\left.\mathrm{CDCl}_{3}\right)$ : $\delta:-104.0(\mathrm{~d}, J=9.8 \mathrm{~Hz}),-109.6(\mathrm{~d}, J=9.7 \mathrm{~Hz})$. HRMS (ESI-TOF) m/z: calc'd for $[\mathrm{M}+\mathrm{H}]^{+}$266.0451, found: 266.0423

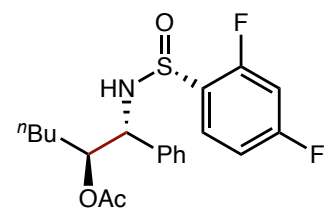

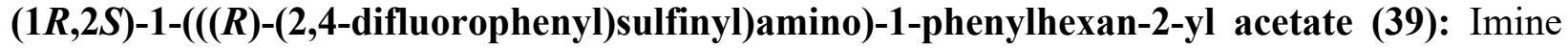
S39 $(53.0 \mathrm{mg}, 0.2 \mathrm{mmol}$ ) was subjected to GP3 and upon column chromatography (20\% ethyl acetate/hexanes) provided 65\% 39 (18:3:3:1 d.r.) as colorless oil. $* \mathbf{1 5} \mathbf{~ m o l \% ~} \mathbf{M n}_{\mathbf{2}}(\mathrm{CO})_{\mathbf{1 0}}$ used. $\mathrm{R}_{\mathrm{f}}$ $=0.2$ (15\% ethyl acetate/hexanes). IR (film): 3650, 2980, 2880, 2250, 1730, 1600, 1480, 1380, 1240, 1140, 1080, 963, 903, 724. ${ }^{1} \mathrm{H}$ NMR (400 MHz, $\left.\mathrm{CDCl}_{3}\right)$ \&: 7.70 (ddd, $J=8.6,8.0,6.3 \mathrm{~Hz}$, $1 \mathrm{H}), 7.15-7.09(\mathrm{~m}, 3 \mathrm{H}), 7.03-6.96(\mathrm{~m}, 2 \mathrm{H}), 6.87-6.80(\mathrm{~m}, 1 \mathrm{H}), 6.45-6.37(\mathrm{~m}, 1 \mathrm{H}), 5.11-$ $5.04(\mathrm{~m}, 2 \mathrm{H}), 4.63-4.59(\mathrm{~m}, 1 \mathrm{H}), 2.06(\mathrm{~s}, 3 \mathrm{H}), 1.53-1.43(\mathrm{~m}, 1 \mathrm{H}), 1.42-1.33(\mathrm{~m}, 1 \mathrm{H}), 1.32-$ $1.27(\mathrm{~m}, 1 \mathrm{H}), 1.23-1.11(\mathrm{~m}, 3 \mathrm{H}), 0.82(\mathrm{t}, J=7.0 \mathrm{~Hz}, 3 \mathrm{H}) .{ }^{13} \mathrm{C} \mathrm{NMR}\left(101 \mathrm{MHz}, \mathrm{CDCl}_{3}\right) \delta: 171.6$, $165.3(\mathrm{dd}, J=254.9,11.3), 159.0(\mathrm{dd}, J=254.1,12.6), 137.9,128.2(\mathrm{dd}, J=13.7,3.3), 128.0$, 127.8, 127.6, $127.1(\mathrm{dd}, J=15.5,3.7), 111.6(\mathrm{dd}, J=22.0,3.5 \mathrm{~Hz}), 104.2(\mathrm{dd}, J=26.3,24.6)$, 76.7, 56.2, 29.9, 27.8, 22.4, 21.2, 14.0. $\left.{ }^{19} \mathrm{~F} \mathrm{NMR} \mathrm{(377} \mathrm{MHz,} \mathrm{CDCl}_{3}\right)$ \&: -105.4 (d, J=10.2 Hz), $108.4\left(\mathrm{~d}, J=10.2 \mathrm{~Hz}\right.$ ). HRMS (ESI-TOF) m/z: calc'd for [M+H] ${ }^{+} 266.0451$, found: 266.0423 . 
<smiles>CC(C)(C)OC(=O)C([18O])NS(=O)(=O)c1ccc(F)cc1F</smiles>

1-((2,4-difluorophenyl)sulfonamido)-1-phenylhexan-2-yl acetate (40): To an oven-dried round bottom flask containing a stir bar, product 39 (1 eq) was dissolved in $\mathrm{CH}_{2} \mathrm{Cl}_{2}(0.2 \mathrm{M})$. Next mCPBA (1.5 eq) was added slowly at rt. Reaction was monitored by TLC. When complete, a 40\% solution of aq. $\mathrm{NaHSO}_{3}(0.2 \mathrm{M})$ was added then stirred for 5 minutes. The mixture was extracted three times with $\mathrm{CH}_{2} \mathrm{Cl}_{2}$. The organic layers were combined and rinsed two times with aq. $\mathrm{HCO}_{3}$ and dried with $\mathrm{Na}_{2} \mathrm{SO}_{4}$. The crude mixture was concentrated and purified by column chromatography $(20 \%$ ethyl acetate/hexanes) to provide product 35\% 40 (98\% ee) as a colorless oil. $\mathrm{R}_{\mathrm{f}}=0.68$ (30\% ethyl acetate/hexanes). IR (film): 3285, 3106, 3065, 3030, 2957, 2931, 2862, 1735, 1602, 1232, 1166, 1073, 969, 753, 701, 537. ${ }^{1} \mathrm{H}$ NMR $\left(400 \mathrm{MHz}, \mathrm{CDCl}_{3}\right) \delta: 7.66(\mathrm{~m}, 1 \mathrm{H}), 7.15(\mathrm{~m}, 3 \mathrm{H}), 7.03(\mathrm{~m}$, 2H), $6.79(\mathrm{~m}, 1 \mathrm{H}), 6.65(\mathrm{~m}, 1 \mathrm{H}), 5.59(\mathrm{~d}, J=4.9,8.9 \mathrm{~Hz}, 1 \mathrm{H}), 5.05(\mathrm{~m}, 1 \mathrm{H}), 4.51(\mathrm{dd}, J=4.9,8.9$ $\mathrm{Hz}, 1 \mathrm{H}), 1.94(\mathrm{~s}, 3 \mathrm{H}), 1.48(\mathrm{~m}, 2 \mathrm{H}), 1.22(\mathrm{~m}, 4 \mathrm{H}), 0.83(\mathrm{t}, J=6.9 \mathrm{~Hz}, 1 \mathrm{H}) .{ }^{13} \mathrm{C} \mathrm{NMR}(101 \mathrm{MHz}$, $\left.\mathrm{CDCl}_{3}\right) \delta: 170.8,165.7(\mathrm{dd}, J=11.5,257.3 \mathrm{~Hz}), 159.3(\mathrm{dd}, J=12.7,257.3 \mathrm{~Hz}), 136.1,131.7(\mathrm{~d}, J$ $=10.5), 128.4,128.1,127.3,125.1(\mathrm{dd}, J=3.8,14.1 \mathrm{~Hz}), 111.5(\mathrm{dd}, J=3.7,21.9 \mathrm{~Hz}), 105.2(\mathrm{t}, J$ $=25.4 \mathrm{~Hz}), 75.9,60.5,30.0,27.5,22.4,20.9,13.9 .{ }^{19} \mathrm{~F} \mathrm{NMR}\left(377 \mathrm{MHz}, \mathrm{CDCl}_{3}\right) \delta:-101.0(\mathrm{~d}, J=$ $12.3 \mathrm{~Hz}$ ), $-104.5\left(\mathrm{~d}, J=12.3 \mathrm{~Hz}\right.$ ). HRMS (ESI-TOF) $\mathrm{m} / \mathrm{z}$ : calc'd for $[\mathrm{M}+\mathrm{H}]^{+} 412.1394$, found: 412.1389 .

HPLC (CHIRALCEL AD-H, 0.46*25 cm, 5 $\mu \mathrm{m}$, hexane/isopropanol = 90/10, flow $0.7 \mathrm{~mL} / \mathrm{min}$, detection at $254 \mathrm{~nm}$ ) retention time $=6.9 \mathrm{~min}$ (major) and $7.4 \mathrm{~min}$ (minor).
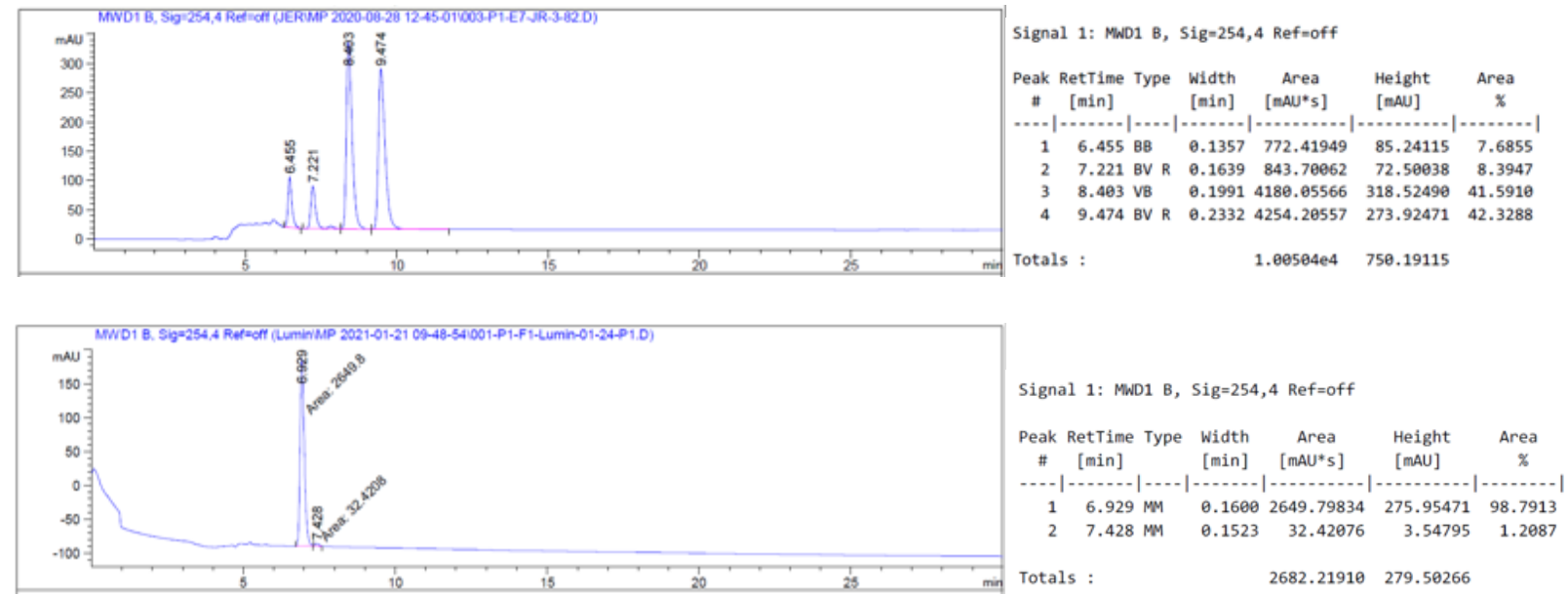


\section{Synthesis and characterization of other ketyl couplings}<smiles>COC(=O)C=CC([18O])OC(C)=O</smiles>

methyl 4-acetoxyoct-2-enoate (41): To an oven dried vial of $\mathrm{CH}_{2} \mathrm{Cl}_{2}(1.0 \mathrm{~mL})$ at $0{ }^{\circ} \mathrm{C}$, $\mathrm{AcI}(20$ $\mu \mathrm{L}, 0.26 \mathrm{mmol})$ was added using a $1 \mathrm{~mL}$ plastic syringe, followed by $\mathrm{Zn}(\mathrm{OTf})_{2}(3.6 \mathrm{mg}, 0.01$ $\mathrm{mmol})$. After dropwise addition of pentanal $(21 \mu \mathrm{L}, 0.2 \mathrm{mmol})$, the reaction was stirred for $15 \mathrm{~min}$ at $0{ }^{\circ} \mathrm{C}$. Next, $\mathrm{Cy}_{2} \mathrm{NMe}(86 \mu \mathrm{L}, 0.4 \mathrm{mmol})$ was added at $0{ }^{\circ} \mathrm{C}$, followed by $\mathrm{Zn}(26.2 \mathrm{mg}, 0.4 \mathrm{mmol})$, Hantzsch Ester $(38.0 \mathrm{mg}, 0.15 \mathrm{mmol})$ and $\mathrm{Mn}_{2}(\mathrm{CO})_{10}(11.4 \mathrm{mg}, 0.03 \mathrm{mmol})$. Finally, methyl propiolate $(89 \mu \mathrm{L}, 1.0 \mathrm{mmol})$ was added and the reaction was degassed by stirring at $-78{ }^{\circ} \mathrm{C}$ for 20 min under vacuum. The reaction was then irradiated with white CFLs for 24 hours. The crude mixture was purified by column chromatography (5-10\% ethyl acetate/hexanes) to provide 56\% 41 (1:1, Z:E) as a slight yellow oil. $\mathrm{R}_{\mathrm{f}}: 0.2$ (5\% ethyl acetate/hexanes). ${ }^{1} \mathrm{H}$ NMR $\left(400 \mathrm{MHz}, \mathrm{CDCl}_{3}\right)$ $\delta$ : E-isomer: $6.85(\mathrm{dd}, J=5.4,15.8 \mathrm{~Hz}, 1 \mathrm{H}), 5.94(\mathrm{dd}, J=1.6,15.8,1 \mathrm{H}), 5.37-5.42(\mathrm{~m}, 1 \mathrm{H})$, $3.74(\mathrm{~s}, 3 \mathrm{H}), 2.09(\mathrm{~s}, 3 \mathrm{H}), 1.63-1.71(\mathrm{~m}, 2 \mathrm{H}), 1.30-1.38(\mathrm{~m}, 4 \mathrm{H}), 0.88-0.92(\mathrm{~m}, 3 \mathrm{H})$. Z-isomer: 6.19 (ddt, $J=1.2,4.9,7.9 \mathrm{~Hz}, 1 \mathrm{H}), 6.07$ (dd, $J=7.9,11.6,1 \mathrm{H}), 5.82(\mathrm{dd}, J=1.2,11.6 \mathrm{~Hz}, 1 \mathrm{H})$, $3.74(\mathrm{~s}, 3 \mathrm{H}), 2.05(\mathrm{~s}, 3 \mathrm{H}), 1.63-1.71(\mathrm{~m}, 2 \mathrm{H}), 1.30-1.38(\mathrm{~m}, 4 \mathrm{H}), 0.88-0.92(\mathrm{~m}, 3 \mathrm{H}) .{ }^{13} \mathrm{C}$ NMR $\left(100 \mathrm{MHz}, \mathrm{CDCl}_{3}\right) \delta$ : $\mathbf{E}$ - and $\mathbf{Z}$ - isomers: $170.5,170.2,166.6,165.9,148.0,145.9,121.2$, 120.0, 72.6, 71.8, 51.8, 51.6, 33.8, 33.6, 27.3, 27.2, 22.6, 22.5, 21.3, 21.1, 14.1, 14.0. IR (film): 2956, 2932, 1735, 1370, 1227. HRMS (ESI-TOF) $\mathrm{m} / \mathrm{z}$ : calc'd for $[\mathrm{M}+\mathrm{Na}]^{+} 237.1103$, found 237.1090 .

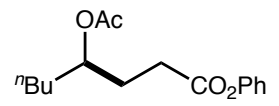

phenyl 4-acetoxyoctanoate (42): To an oven dried vial of $\mathrm{CH}_{2} \mathrm{Cl}_{2}(1.0 \mathrm{~mL})$ at $0{ }^{\circ} \mathrm{C}, \mathrm{AcI}(40 \mu \mathrm{L}$, $0.52 \mathrm{mmol})$ was added using a $1 \mathrm{~mL}$ plastic syringe. After dropwise addition of pentanal $(64 \mu \mathrm{L}$, $0.6 \mathrm{mmol})$, the reaction was stirred for $15 \mathrm{~min}$ at $0{ }^{\circ} \mathrm{C}$. Next, $\mathrm{Cy}_{2} \mathrm{NMe}(89 \mu \mathrm{L}, 0.4 \mathrm{mmol})$ was at 0 ${ }^{\circ} \mathrm{C}$, followed by $\mathrm{Zn}(26.2 \mathrm{mg}, 0.4 \mathrm{mmol})$, Hantzsch Ester $(38.0 \mathrm{mg}, 0.15 \mathrm{mmol})$ and $\mathrm{Mn}_{2}(\mathrm{CO})_{10}$ $(11.4 \mathrm{mg}, 0.03 \mathrm{mmol})$, then phenyl acrylate $(32 \mu \mathrm{L}, 0.2 \mathrm{mmol})$. The reaction was degassed by stirring at $-78^{\circ} \mathrm{C}$ for 20 min under vacuum. The reaction was then irradiated with white CFLs for 24 hours. The crude mixture was purified by column chromatography ( $3 \%$ ethyl acetate/hexanes) to provide $85 \% 42$ as a colorless oil. $\mathrm{R}_{\mathrm{f}}$ : 0.2 (5\% ethyl acetate/hexanes). ${ }^{1} \mathrm{H}$ NMR (400 MHz, $\left.\mathrm{CDCl}_{3}\right) \delta: 7.35-7.39(\mathrm{~m}, 2 \mathrm{H}), 7.20-7.26(\mathrm{~m}, 1 \mathrm{H}), 7.08-7.11(\mathrm{~m}, 1 \mathrm{H}), 4.95-5.02(\mathrm{~m}, 1 \mathrm{H}), 2.59$ $(\mathrm{t}, J=7.5 \mathrm{~Hz}, 2 \mathrm{H}), 2.06(\mathrm{~s}, 3 \mathrm{H}), 1.90-2.11(\mathrm{~m}, 2 \mathrm{H}), 1.51-1.67(\mathrm{~m}, 4 \mathrm{H}), 0.90(\mathrm{t}, J=6.9 \mathrm{~Hz}, 3 \mathrm{H})$. ${ }^{13} \mathrm{C}$ NMR $\left(100 \mathrm{MHz}, \mathrm{CDCl}_{3}\right) \delta: 171.8,171.0,150.8,129.6,126.0,121.7,73.3,34.0,30.6,29.3$, 27.6, 22.7, 21.3, 14.1. IR (film): 2958, 2932, 2862, 1735, 1370. HRMS (ESI-TOF) m/z: calc'd for $[\mathrm{M}+\mathrm{Na}]^{+}$301.1416, found 301.1398. 


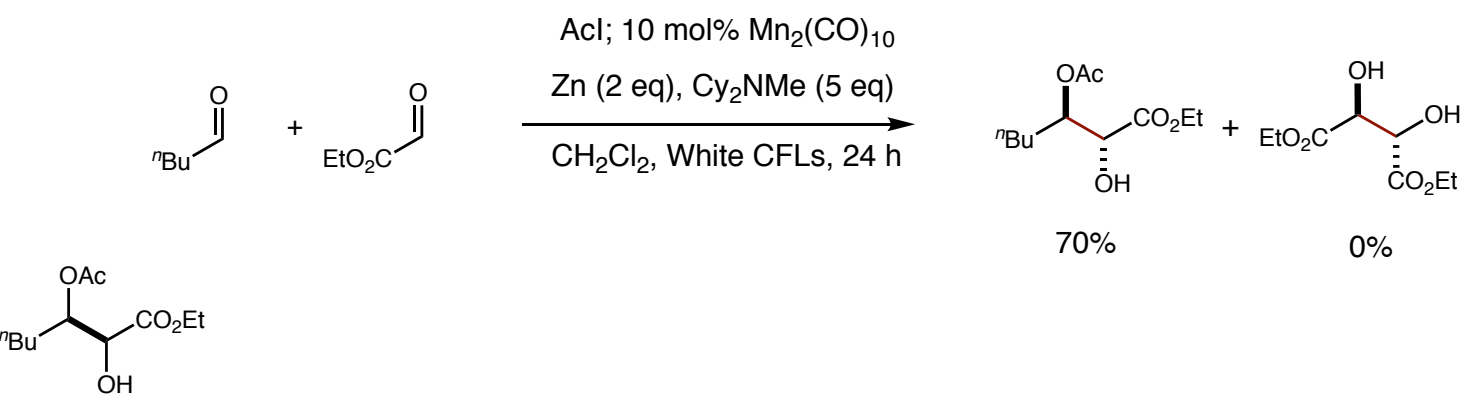

ethyl 3-acetoxy-2-hydroxyheptanoate (43): To an oven dried vial of $\mathrm{CH}_{2} \mathrm{Cl}_{2}(1.0 \mathrm{~mL})$ at $0{ }^{\circ} \mathrm{C}$, AcI $(20 \mu \mathrm{L}, 0.26 \mathrm{mmol})$ was added using a $1 \mathrm{~mL}$ plastic syringe. After dropwise addition of pentanal $(64 \mu \mathrm{L}, 0.6 \mathrm{mmol})$, the reaction was stirred for $15 \mathrm{~min}$ at $0{ }^{\circ} \mathrm{C}$. Next, $\mathrm{Cy}_{2} \mathrm{NMe}(214 \mu \mathrm{L}$, $1.0 \mathrm{mmol})$ was added at $0{ }^{\circ} \mathrm{C}$, followed by $\mathrm{Zn}(26.2 \mathrm{mg}, 0.4 \mathrm{mmol})$ and $\mathrm{Mn}_{2}(\mathrm{CO})_{10}(7.8 \mathrm{mg}, 0.02$ $\mathrm{mmol}$ ), and freshly distilled ethyl glyoxylate ( $40 \mu \mathrm{L}, 0.2 \mathrm{mmol}, 50 \%$ soln. in toluene). The reaction was degassed by stirring at $-78^{\circ} \mathrm{C}$ for 20 min under vacuum. The reaction was then irradiated with white CFLs for 24 hours. The crude mixture was purified by column chromatography ( $25 \%$ ethyl acetate/hexanes) to provide $70 \% \mathbf{4 3}$ as a colorless oil. $\mathrm{R}_{\mathrm{f}}$ : $0.23,0.20$ (15\% ethyl acetate/hexanes). IR (film): 2957, 2932, 2861, 1735, 1230, 1022. HRMS (ESI-TOF) m/z: calc'd for $[\mathrm{M}+\mathrm{Na}]^{+}$ 255.1208 , found 255.1196 .

Diastereomer A: ${ }^{1} \mathrm{H}$ NMR $\left(400 \mathrm{MHz}, \mathrm{CDCl}_{3}\right) \delta: 5.16(\mathrm{dt}, J=2.1,7.2 \mathrm{~Hz}, 1 \mathrm{H}), 4.20-4.25(\mathrm{~m}$, 2H), 4.19 (dd, $J=2.1,7.8 \mathrm{~Hz}, 1 \mathrm{H}), 2.86$ (d, $J=7.8 \mathrm{~Hz}, 1 \mathrm{H}), 2.03$ (s, 3H), $1.71-1.76(\mathrm{~m}, 2 \mathrm{H})$, $1.31-1.37(\mathrm{~m}, 4 \mathrm{H}), 1.28(\mathrm{t}, J=7.1 \mathrm{~Hz}, 3 \mathrm{H}), 0.91$ (t, $J=7.1 \mathrm{~Hz}, 3 \mathrm{H}) .{ }^{13} \mathrm{C} \mathrm{NMR}\left(100 \mathrm{MHz}, \mathrm{CDCl}_{3}\right)$ $\delta: 172.9,170.3,74.4,71.6,62.2,30.3,27.6,22.6,20.9,14.2,14.1$.

Diastereomer B: ${ }^{1} \mathrm{H}$ NMR $\left(400 \mathrm{MHz}, \mathrm{CDCl}_{3}\right) \delta: 5.11(\mathrm{dt}, J=9.3,3.9 \mathrm{~Hz}, 1 \mathrm{H}), 4.26-4.31(\mathrm{~m}$, $3 \mathrm{H}), 3.05(\mathrm{~d}, J=6.1 \mathrm{~Hz}, 1 \mathrm{H}), 2.09(\mathrm{~s}, 3 \mathrm{H}), 1.71-1.78(\mathrm{~m}, 2 \mathrm{H}), 1.49-1.53(\mathrm{~m}, 2 \mathrm{H}), 1.26-1.30$ $(\mathrm{m}, 2 \mathrm{H}), 1.32(\mathrm{t}, J=7.1 \mathrm{~Hz}, 3 \mathrm{H}), 0.89(\mathrm{t}, J=7.0 \mathrm{~Hz}, 3 \mathrm{H}) .{ }^{13} \mathrm{C} \mathrm{NMR}\left(100 \mathrm{MHz}, \mathrm{CDCl}_{3}\right) \delta: 172.4$, $171.0,75.2,72.2,62.4,29.0,27.6,22.6,21.2,14.3,14.0$. 
Single-electron transfer (SET) strategy:

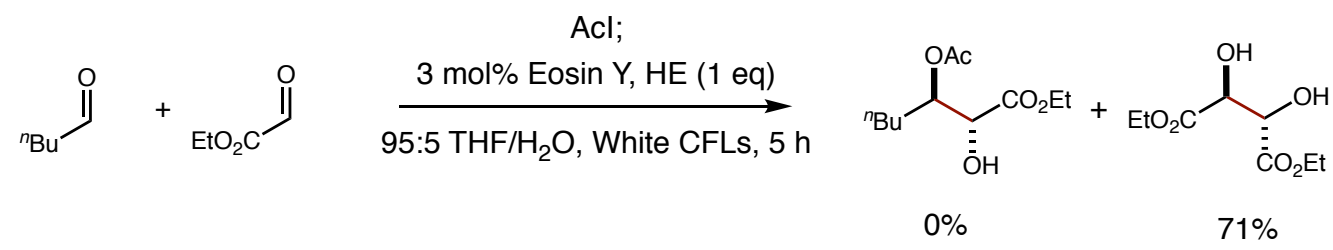

$\mathrm{EtO}_{2} \mathrm{C} \overbrace{\mathrm{OH}}^{\mathrm{OH}} \mathrm{CO}_{2} \mathrm{Et}$

diethyl 2,3-dihydroxysuccinate (44): Using Zhu et al's reductive procedure ${ }^{13}$, a vial with $\mathrm{Na}_{2} \mathrm{Ey}$ (4.9 $\mathrm{mg}, 0.006 \mathrm{mmol})$ and Hantzsch Ester $(41.8 \mathrm{mg}, 0.165 \mathrm{mmol})$ was dissolved in degassed 95:5 $\mathrm{THF} / \mathrm{H}_{2} \mathrm{O}(6 \mathrm{~mL}),{ }^{\mathrm{n}} \mathrm{BuC}(\mathrm{OAc}) \mathrm{I}(54 \mu \mathrm{L}, 0.3 \mathrm{mmol})$ and freshly distilled ethyl glyoxylate $(0.3$ $\mathrm{mmol})$ in toluene was added. The reaction mixture was irradiated with $23 \mathrm{~W}$ white CFLs for 5 hours. The reaction was extracted with ethyl acetate, washed with brine, then dried with sodium sulfate. The yield of diethyl tartrate 44 was determined by NMR (71\% yield). ${ }^{1} \mathrm{H}$ NMR (400 MHz, $\left.\mathrm{CDCl}_{3}\right) \delta: 4.53(\mathrm{~d}, J=6.4 \mathrm{~Hz}, 2 \mathrm{H}), 4.33(\mathrm{q}, J=7.1 \mathrm{~Hz}, 4 \mathrm{H}), 3.14(\mathrm{~d}, J=6.9 \mathrm{~Hz}, 2 \mathrm{H}), 1.33(\mathrm{t}, J=$ $7.1 \mathrm{~Hz}, 6 \mathrm{H}) .{ }^{13} \mathrm{C} \mathrm{NMR}\left(100 \mathrm{MHz}, \mathrm{CDCl}_{3}\right) \delta: 171.7,72.1,62.6,14.3$. HRMS (ESI-TOF) m/z: calc'd for $[\mathrm{M}+\mathrm{Na}]^{+} 229.0688$, found 229.0681 .

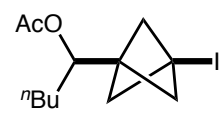

1-(3-iodobicyclo[1.1.1]pentan-1-yl)pentyl acetate (45): To an oven dried vial of $\mathrm{CH}_{2} \mathrm{Cl}_{2}(1.0$ $\mathrm{mL})$ at $0{ }^{\circ} \mathrm{C}$, AcI $(20 \mu \mathrm{L}, 0.26 \mathrm{mmol})$ was added using a $1 \mathrm{~mL}$ plastic syringe, followed by $\mathrm{Zn}(\mathrm{OTf})_{2}(3.6 \mathrm{mg}, 0.01 \mathrm{mmol})$. After dropwise addition of pentanal $(21 \mu \mathrm{L}, 0.2 \mathrm{mmol})$, the reaction was stirred for $15 \mathrm{~min}$ at $0{ }^{\circ} \mathrm{C}$. Next, ${ }^{i} \operatorname{Pr}_{2} \mathrm{NEt}(17 \mu \mathrm{L}, 0.1 \mathrm{mmol})$ was added at $0{ }^{\circ} \mathrm{C}$, followed by $\mathrm{Mn}_{2}(\mathrm{CO})_{10}(7.8 \mathrm{mg}, 0.02 \mathrm{mmol})$. Finally, methyl propiolate $(89 \mu \mathrm{L}, 1.0 \mathrm{mmol})$ was added and the reaction was degassed by stirring in dry ice/diethyl ether under vacuum for $20 \mathrm{~min}$. The reaction was then irradiated with one $90 \mathrm{~W}$ blue LED lamp (Kessil A360WE tuna blue), then the crude mixture was purified by column chromatography (5\% ethyl acetate/hexanes) to provide $67 \% 45$ as a slight yellow oil. $\mathrm{R}_{\mathrm{f}}$ : 0.41 (5\% ethyl acetate/hexanes). ${ }^{1} \mathrm{H}$ NMR $\left(400 \mathrm{MHz}, \mathrm{CDCl}_{3}\right) \delta$ : $4.84(\mathrm{t}, J=6.7 \mathrm{~Hz}, 1 \mathrm{H}), 2.23(\mathrm{~s}, 6 \mathrm{H}), 2.04(\mathrm{~s}, 3 \mathrm{H}), 1.42-1.47(\mathrm{~m}, 2 \mathrm{H}), 1.25-1.31(\mathrm{~m}, 4 \mathrm{H}), 0.88$ $(\mathrm{t}, J=7.0 \mathrm{~Hz}, 3 \mathrm{H}) .{ }^{13} \mathrm{C} \mathrm{NMR}\left(100 \mathrm{MHz}, \mathrm{CDCl}_{3}\right) \delta: 170.6,72.3,58.9,49.5,31.3,27.7,22.5,21.0$, 14.0, 6.9. HRMS (ESI-TOF) $\mathrm{m} / \mathrm{z}$ : calc'd for $[\mathrm{M}+\mathrm{Na}]^{+} 345.0327$, found 345.0359 . 


\section{Determination of stereochemistry}

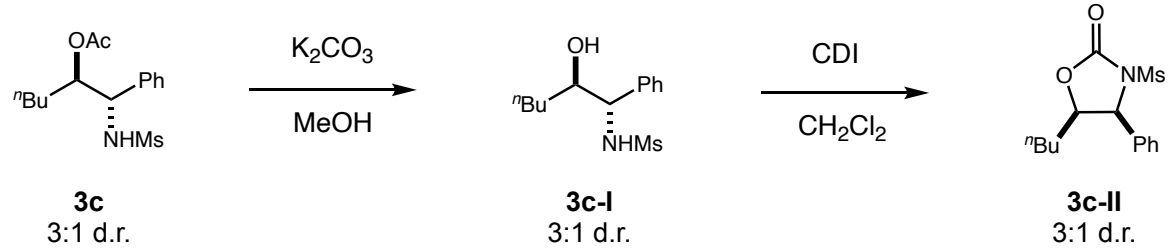

Aza-pinacol adduct 3c was saponified to 3c-I, then cyclized to oxazolidinone 3c-II. NOESY experiments confirm the major isomer of the cyclic adduct 3c-II has a syn arrangement, which corresponds to anti arrangements of product $\mathbf{3 c}$ and free alcohol $\mathbf{3 c}-\mathbf{I}$.

Stereochemical correlation: All $>2: 1 \mathrm{dr}$ products in Table 2 are assigned as anti, since the $\alpha$ amino C-H of the major isomer (anti: $4.7 \mathrm{ppm}$ ) is always downfield of the minor isomer (syn: $\sim 4.5 \mathrm{ppm}$ ). Additionally, coupling constants $(\mathrm{J})$ are smaller for the major isomer (anti) versus the minor (syn) isomer.<smiles>NC(c1ccccc1)C(O)[18OH]</smiles>

$\boldsymbol{N}$-(2-hydroxy-1-phenylhexyl)methanesulfonamide (3c-I): To amino acetate 3c $(62.7 \mathrm{mg}, 0.2$ $\mathrm{mmol})$ and $\mathrm{K}_{2} \mathrm{CO}_{3}(30.4 \mathrm{mg}, 0.22 \mathrm{mmol}), \mathrm{MeOH}(1 \mathrm{~mL})$ was added. Upon completion, solvent was removed and the crude mixture was subjected to column chromatography (30\% ethyl acetate/hexanes) to provide $85 \%$ 3c-I (3:1 d.r) as a white solid. HRMS (ESI-TOF) m/z: calc'd for $[\mathrm{M}+\mathrm{Na}]^{+} 294.1140$, found 294.1118 .

Major diastereomer: ${ }^{1} \mathrm{H}$ NMR $\left(400 \mathrm{MHz}, \mathrm{CDCl}_{3}\right) \delta: 7.29-7.40(\mathrm{~m}, 5 \mathrm{H}), 5.93(\mathrm{~d}, J=8.9 \mathrm{~Hz}$, $1 \mathrm{H}), 4.46(\mathrm{dd}, J=3.7,9.0,1 \mathrm{H}), 3.96-3.98(\mathrm{~m}, 1 \mathrm{H}), 2.57(\mathrm{~s}, 3 \mathrm{H}), 2.27$ (brs, $1 \mathrm{H}), 1.07-1.49$ (m, $6 \mathrm{H}), 0.84-0.87(\mathrm{~m}, 1 \mathrm{H}) .{ }^{13} \mathrm{C} \mathrm{NMR}\left(100 \mathrm{MHz}, \mathrm{CDCl}_{3}\right) \delta: 137.3,128.8,128.2,127.4,74.8,61.8$, $41.8,33.5,28.0,22.6,14.0$.

Minor diastereomer: ${ }^{1} \mathrm{H}$ NMR $\left(400 \mathrm{MHz}, \mathrm{CDCl}_{3}\right) \delta: 7.29-7.40(\mathrm{~m}, 5 \mathrm{H}), 5.83(\mathrm{~d}, J=7.5 \mathrm{~Hz}$, $1 \mathrm{H}), 4.37$ (dd, $J=5.7,7.4 \mathrm{~Hz}, 1 \mathrm{H}), 3.71-3.75(\mathrm{~m}, 1 \mathrm{H}), 2.60$ (s, 3H), 1.93 (brs, $1 \mathrm{H}), 1.07-1.49$ $(\mathrm{m}, 6 \mathrm{H}), 0.84-0.87(\mathrm{~m}, 1 \mathrm{H}) .{ }^{13} \mathrm{C} \mathrm{NMR}\left(100 \mathrm{MHz}, \mathrm{CDCl}_{3}\right) \delta: 139.5,129.1,128.3,128.3,74.9$, 62.6, 42.0, 33.6, 27.8, 22.6, 14.0.<smiles>O=C1OC([18OH])C([18OH])[C@@H]1c1ccccc1</smiles>

5-butyl-3-(methylsulfonyl)-4-phenyloxazolidin-2-one (3c-II): To amino alcohol 3c-I (46.1 mg, $0.17 \mathrm{mmol}$ ) and 1,1'-carbonyldiimidazole (55.2 $\mathrm{mg}, 0.34 \mathrm{mmol}), \mathrm{CH}_{2} \mathrm{Cl}_{2}(2 \mathrm{~mL})$ was added. Upon completion, solvent was removed and the crude mixture was subjected to column chromatography to provide $92 \%$ 3c-II $\left(3: 1\right.$ d.r.) as a white solid. HRMS (ESI-TOF) m/z: calc'd for $[\mathrm{M}+\mathrm{Na}]^{+}$ 320.0932, found 320.0942.

Major diastereomer (Syn): ${ }^{1} \mathrm{H}$ NMR $\left(400 \mathrm{MHz}, \mathrm{CDCl}_{3}\right) \delta: 7.42-7.44(\mathrm{~m}, 3 \mathrm{H}), 7.26$ - $7.28(\mathrm{~m}$, 2H), 5.29 (d, $J=7.1 \mathrm{~Hz}, 1 \mathrm{H}), 4.84-4.88(\mathrm{~m}, 1 \mathrm{H}), 2.98(\mathrm{~s}, 3 \mathrm{H}), 1.36-1.41(\mathrm{~m}, 2 \mathrm{H}), 1.17-1.23$ 
(m, 4H), $0.78(\mathrm{t}, J=7.2 \mathrm{~Hz}, 3 \mathrm{H}) .{ }^{13} \mathrm{C} \mathrm{NMR}\left(100 \mathrm{MHz}, \mathrm{CDCl}_{3}\right) \delta: 152.9,133.9,129.8,129.3$, 123.0, 80.9, 64.1, 41.4, 29.7, 27.4, 22.2, 13.8 .

Minor diastereomer (Anti): ${ }^{1} \mathrm{H}$ NMR $\left(400 \mathrm{MHz}, \mathrm{CDCl}_{3}\right) \delta$ : $7.42-7.44(\mathrm{~m}, 3 \mathrm{H}), 7.36-7.37(\mathrm{~m}$, $2 \mathrm{H}), 4.95(\mathrm{~d}, J=3.6 \mathrm{~Hz}, 1 \mathrm{H}), 4.46-4.49(\mathrm{~m}, 1 \mathrm{H}), 3.00(\mathrm{~s}, 3 \mathrm{H}), 1.77-1.93(\mathrm{~m}, 2 \mathrm{H}), 1.35-1.40$ $(\mathrm{m}, 2 \mathrm{H}), 1.18-1.21(\mathrm{~m}, 2 \mathrm{H}), 0.92(\mathrm{t}, J=6.5 \mathrm{~Hz}, 1 \mathrm{H}) .{ }^{13} \mathrm{C} \mathrm{NMR}\left(100 \mathrm{MHz}, \mathrm{CDCl}_{3}\right) \delta: 152.5$, 138.0, 129.7, 127.9, 126.7, 83.6, 65.0, 41.6, 34.3, 26.5, 22.3, 13.9.

\section{NOESY Experiments}

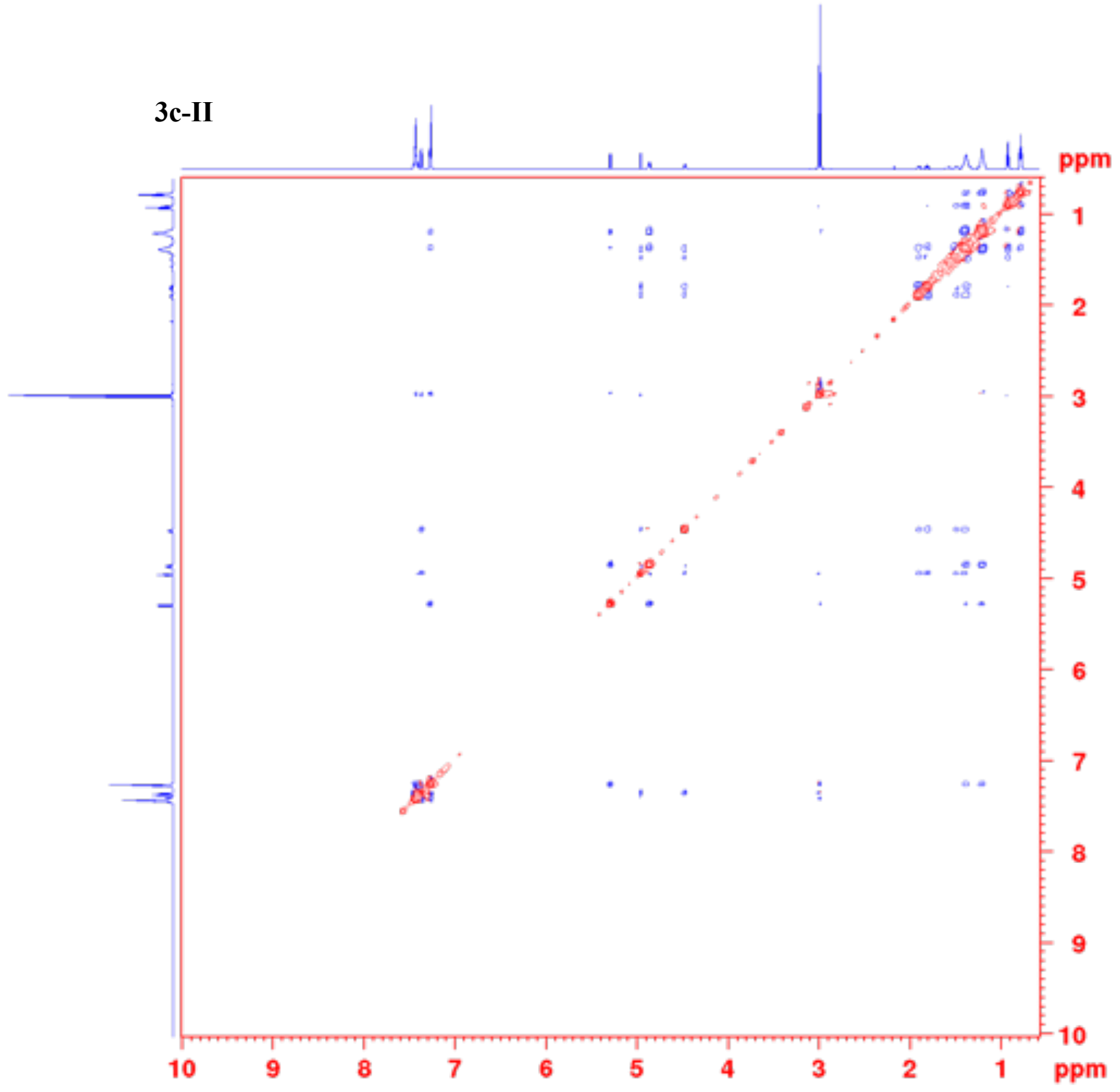




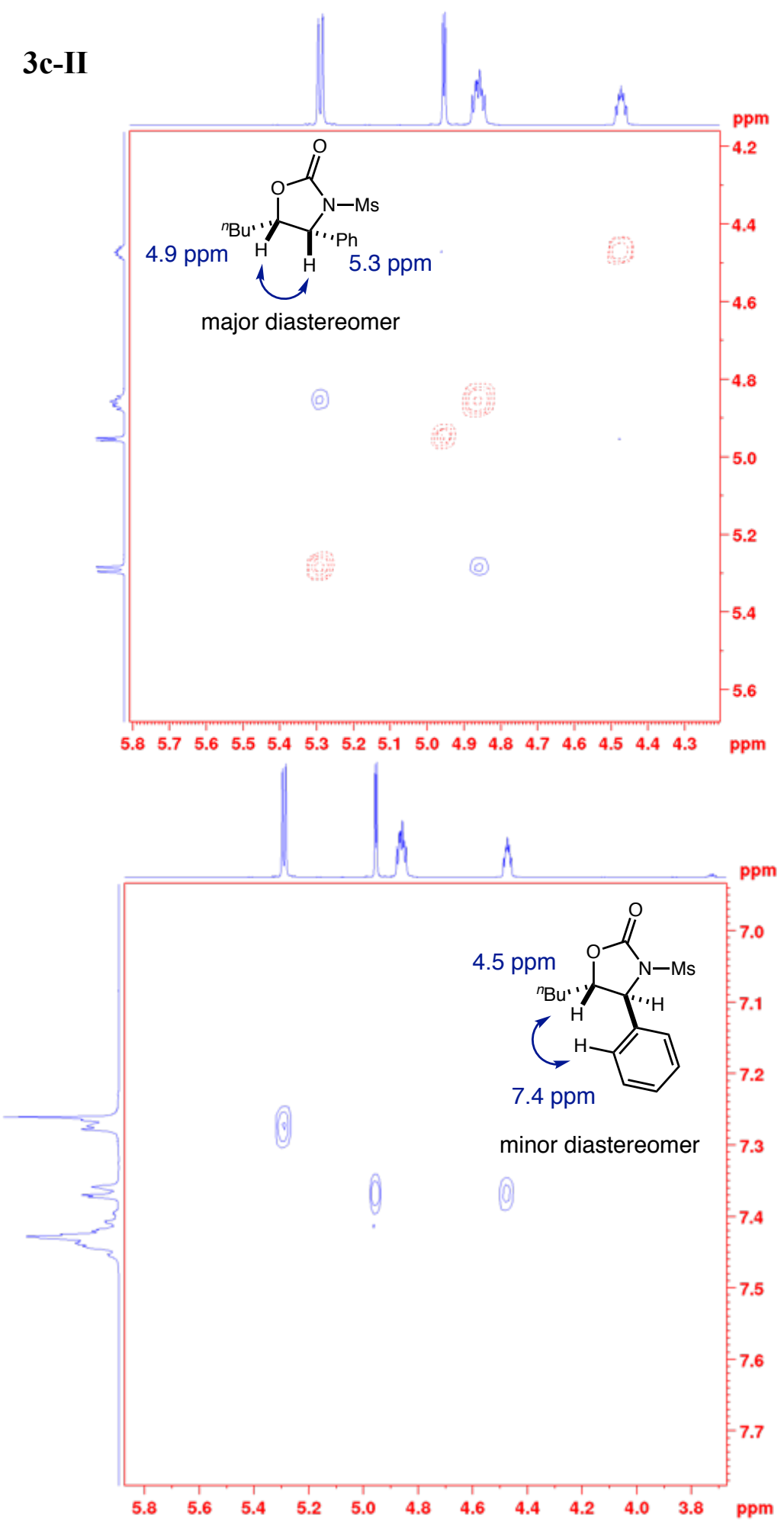

A NOESY correlation was only observed between the $\alpha$-amino C-H (5.3 ppm) and $\alpha$-oxy C-H (4.9 ppm) of the major isomer (top). Additionally, the ortho-phenyl C-H (7.4 ppm) only correlates to the $\alpha$-oxy C-H (4.5 ppm) of the minor isomer (bottom), as well as to the $\alpha$-amino C-H of each. 


\section{Mechanistic insights}

\section{Zn-mediated turnover of Mn catalyst}

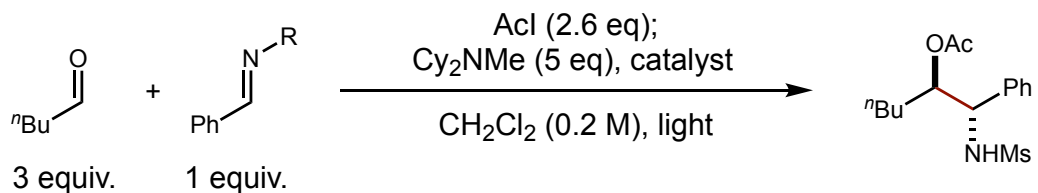

$\begin{array}{ccccc}\text { entry } & \text { catalyst } & \text { light } & \mathrm{Zn} & \text { yield, d.r. } \\ 1 & 5 \% \mathrm{Mn}(\mathrm{CO})_{10} & + & + & 90 \%, 3: 1 \\ 2 & 10 \%(\mathrm{CO})_{5} \mathrm{Mn}-\mathrm{I} & + & + & 66 \%, 3: 1 \\ 3 & 10 \%(\mathrm{CO})_{5} \mathrm{Mn}-\mathrm{I} & + & - & 25 \%, 3: 1 \\ 4 & 10 \%(\mathrm{CO})_{5} \mathrm{Mn}-\mathrm{I} & - & + & 51 \%, 3: 1\end{array}$

Table S2. Mechanistic insight of $\mathrm{Zn}$ turnover of Mn-I via reaction controls.

\section{Investigation of the amine role in radical termination}

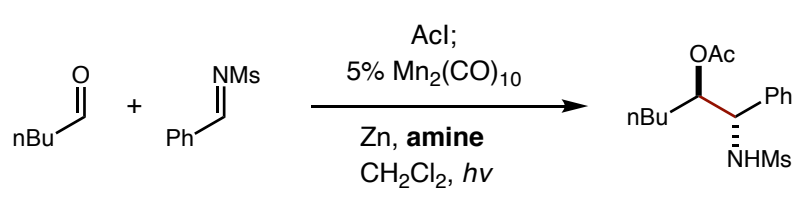

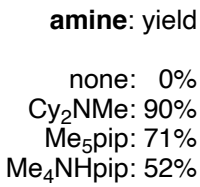

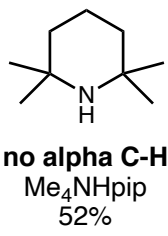

To an oven dried vial of $\mathrm{CH}_{2} \mathrm{Cl}_{2}(1.0 \mathrm{~mL})$ at $0{ }^{\circ} \mathrm{C}$, AcI $(40 \mu \mathrm{L}, 0.52 \mathrm{mmol})$ was added using a 1 $\mathrm{mL}$ plastic syringe. After dropwise addition of aldehyde $(0.6 \mathrm{mmol})$, the reaction was stirred for $15 \mathrm{~min}$ at $0{ }^{\circ} \mathrm{C}$. Next, amine $(1.0 \mathrm{mmol})$ was added at $0{ }^{\circ} \mathrm{C}$, followed by imine $2 \mathrm{c}(36.6 \mathrm{mg}, 0.2$ $\mathrm{mmol}), \mathrm{Zn}(26.2 \mathrm{mg}, 0.4 \mathrm{mmol})$, and $\mathrm{Mn}_{2}(\mathrm{CO})_{10}(3.9 \mathrm{mg}, 0.01 \mathrm{mmol}, 5 \mathrm{~mol} \%)$. The reaction was degassed by stirring at $-78{ }^{\circ} \mathrm{C}$ for 20 min under vacuum. The reaction was then irradiated with white CFLs for 24 hours. The reaction mixture was passed through a silica plug using $10 \%$ ethyl acetate/hexanes $(50 \mathrm{~mL})$ to remove residual $\mathrm{Mn}_{2}(\mathrm{CO})_{10}$. EtOAc $(50 \mathrm{~mL})$ was passed through the silica and the reaction mixture was concentrated. Yield was determined by ${ }^{1} \mathrm{H}$ NMR which was determined by using $\mathrm{CH}_{2} \mathrm{Br}_{2}(7 \mu \mathrm{L}, 0.1 \mathrm{mmol})$ as an internal standard.

Based on this data, we confirmed both $\mathrm{Zn}$ and amine are necessary for reactivity, but while an $\alpha$ amino C-H improves efficiency, likely via termination by HAT to the N-radical (e.g. $\mathrm{Cy}_{2} \mathrm{NMe}$, pentamethylpiperidine), it is not essential, and other amines (e.g. tetramethylpiperidine) may assist with the $\mathrm{Zn}$-mediated reduction. 


\section{UV-Vis experiments}

To catalyst $(0.1 \mathrm{mmol})$ and $\mathrm{Zn}(13.1 \mathrm{mg}, 0.2 \mathrm{mmol})$, degassed $\mathrm{CH}_{2} \mathrm{Cl}_{2}(1 \mathrm{~mL})$ was added, then $\mathrm{Cy}_{2} \mathrm{NMe}(0.11 \mathrm{~mL}, 0.5 \mathrm{mmol})$. The reaction was irradiated with two $23 \mathrm{~W}$ (1650 lumens) white CFL lights for 2 hours. A $200 \mu \mathrm{L}$ aliquot was then diluted to $10 \mathrm{~mL}$ to give the spectra below. UV/Vis analysis was performed with a Cary 5000 UV-Vis/NIR spectrophotometer.

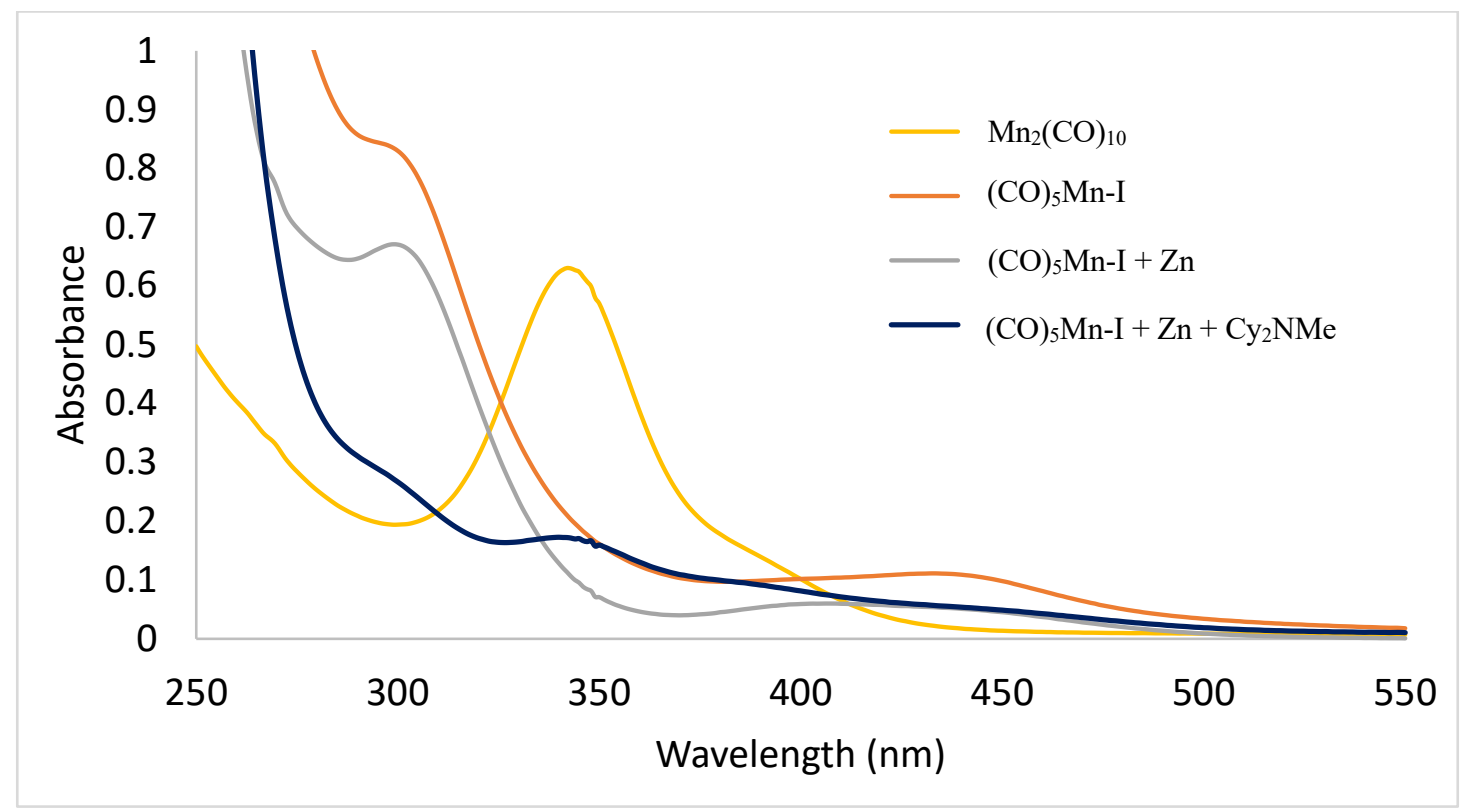

Figure S2: UV-Vis absorbance to observe conversion of $\mathrm{Mn}_{2}(\mathrm{CO})_{10}$ to $(\mathrm{CO})_{5} \mathrm{Mn}-\mathrm{I}$ with $\mathrm{Zn}$ and $\mathrm{Cy}_{2} \mathrm{NMe}$.

To catalyst $(0.1 \mathrm{mmol})$, degassed $\mathrm{CH}_{2} \mathrm{Cl}_{2}(1 \mathrm{~mL})$ was added, then $\mathrm{Cy}_{2} \mathrm{NMe}(0 \mu \mathrm{L}, 0 \mathrm{mmol} ; 4.3$ $\mu \mathrm{L}, 0.02 \mathrm{mmol} ; 10.7 \mu \mathrm{L}, 0.05 \mathrm{mmol} ; 21.4 \mu \mathrm{L}, 0.1 \mathrm{mmol} ; 107.1 \mu \mathrm{L}, 0.5 \mathrm{mmol})$. A $200 \mu \mathrm{L}$ aliquot was then diluted to $10 \mathrm{~mL}$ to give the below spectrum. 


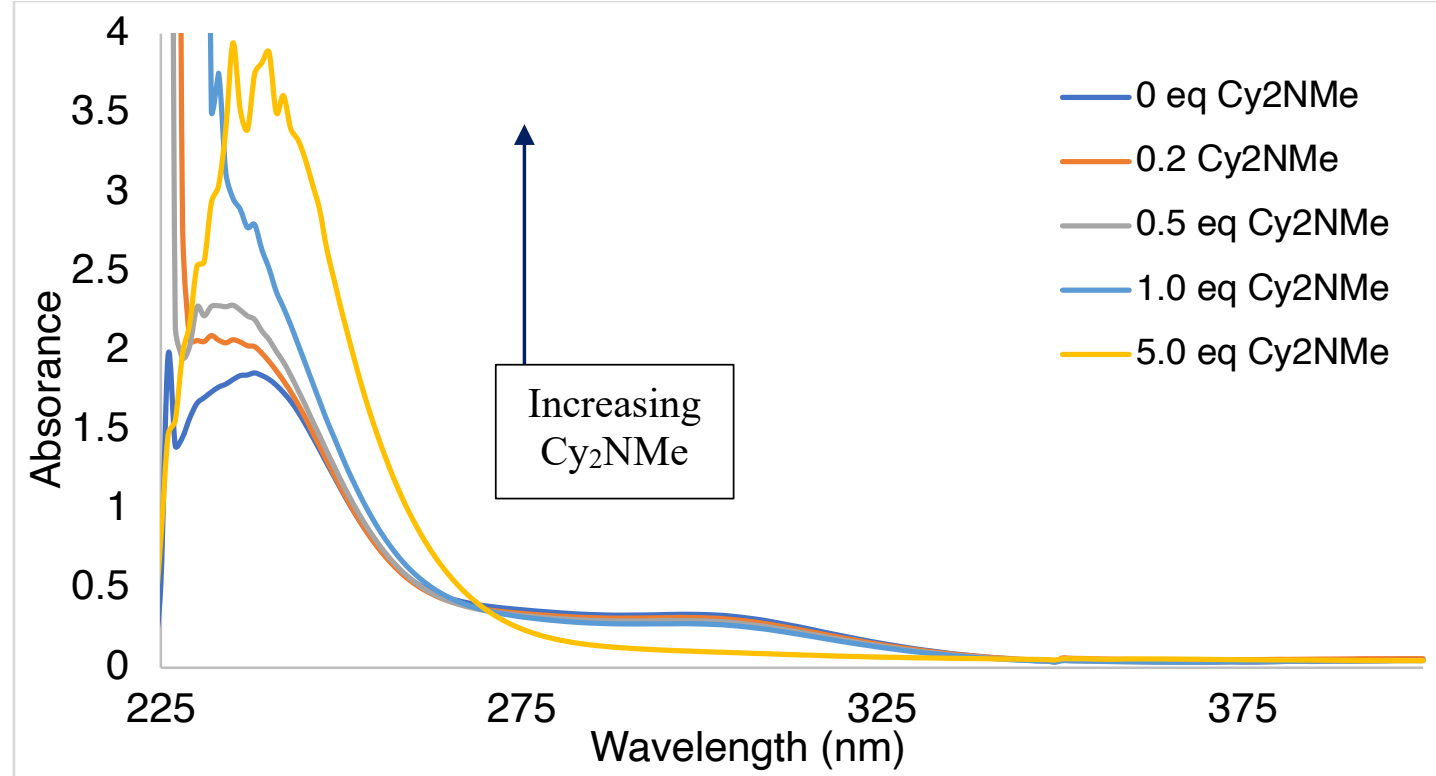

Figure S3: Increasing amounts of $\mathrm{Cy}_{2} \mathrm{NMe}$ with $(\mathrm{CO})_{5} \mathrm{MnI}, \lambda=225-400 \mathrm{~nm}$

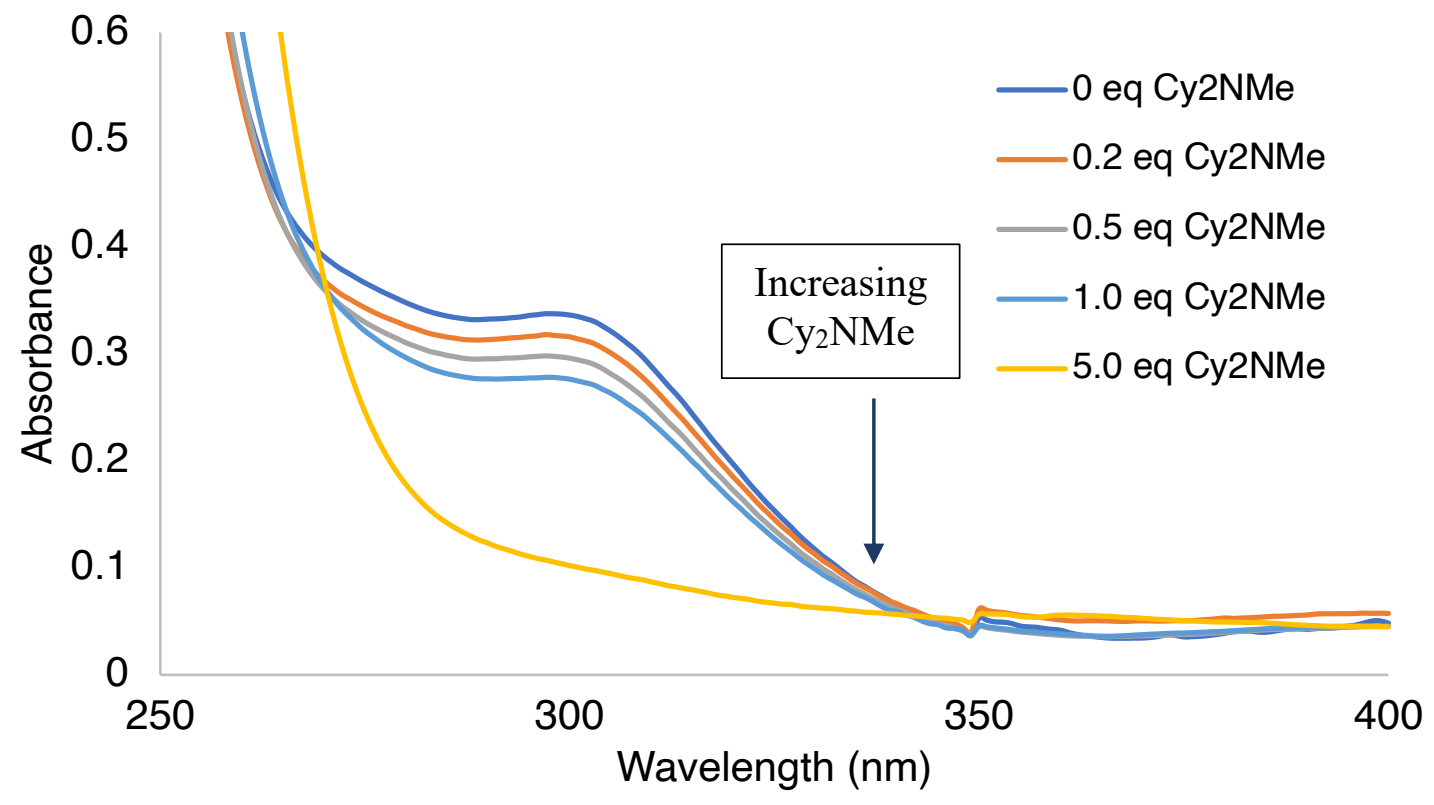

Figure S4: Increasing amounts of $\mathrm{Cy}_{2} \mathrm{NMe}$ with $(\mathrm{CO})_{5} \mathrm{MnI}$ results in decrease of $(\mathrm{CO})_{5} \mathrm{MnI}$ absorbance $(\lambda=300 \mathrm{~nm})$ 


\section{Cyclic voltammetry experiments}

Cyclic voltammograms were recorded using Gamry Reference 600 Potentiostat. A glassy carbon disk (3 mm diameter, $\mathrm{CH}$ Instrument, part \# CHI104), Pt wire, and nonaqueous Ag/Ag+ electrode was used as the working, counter and reference electrode, respectively. The nonaqueous $\mathrm{Ag} / \mathrm{Ag}+$ electrode was prepared in an electrode kit ( $\mathrm{CH}$ instrument, part \# $\mathrm{CH} 112)$ in which a silver wire was immersed into an $\mathrm{MeCN}$ solution containing $0.01 \mathrm{M} \mathrm{AgNO}_{3}$ and $0.1 \mathrm{M}\left[{ }^{n} \mathrm{Bu}_{4} \mathrm{~N}\right] \mathrm{PF}_{6}$. The electrolyte solution was $1 \mathrm{mM}$ substrate/ $1 \mathrm{mM}\left[{ }^{n} \mathrm{Bu}_{4} \mathrm{~N}\right] \mathrm{PF}_{6}$ in anhydrous $\mathrm{CH}_{2} \mathrm{Cl}_{2}$ and was bubbled with argon for 20 minutes prior to the measurement. A scan rate of $100 \mathrm{mV} / \mathrm{s}$ was used.

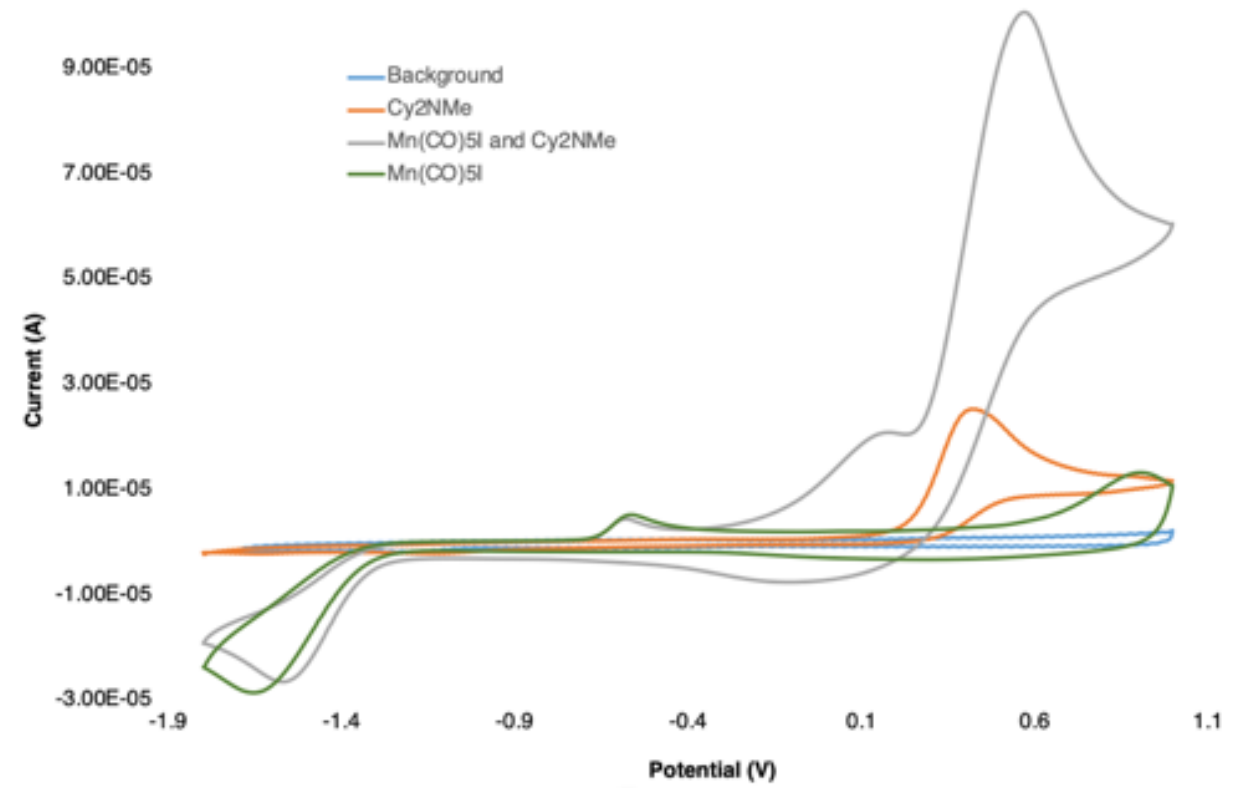

Figure S5: Cyclic voltammogram of manganese catalysts

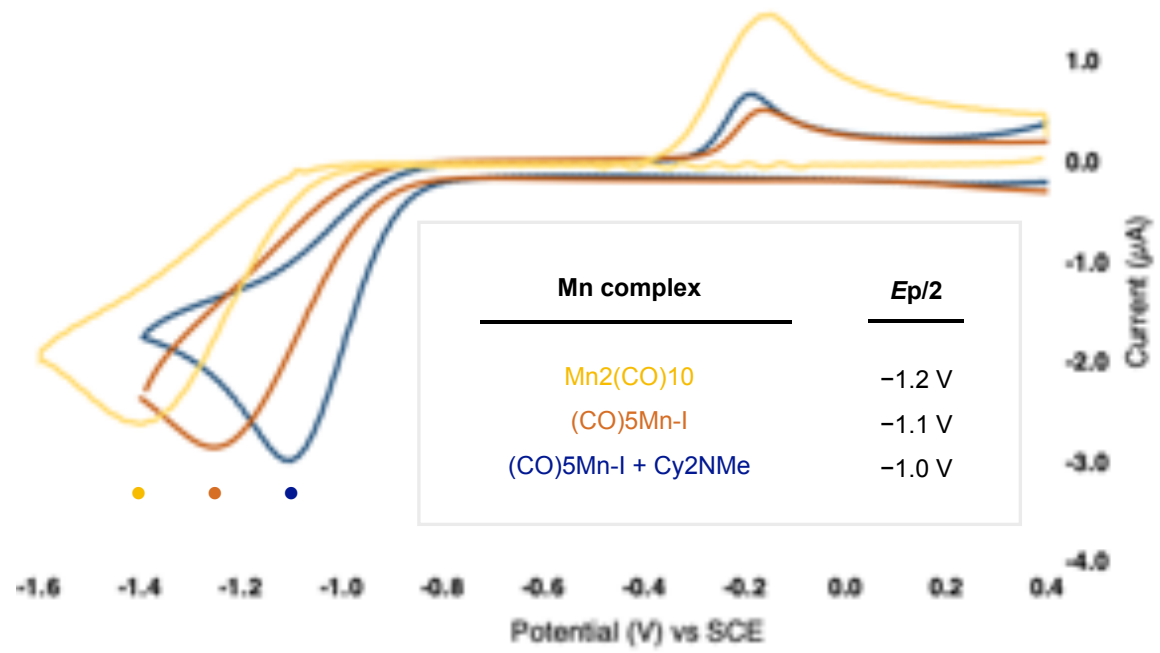

Figure S6: Cyclic voltammogram of manganese catalysts and influence of amine. 


\section{References}

(1) Chen, D.; Chen, X.; Du, T.; Kong, L.; Zhen, R.; Zhen, S.; Wen, Y.; Zhu, G. Highly Efficient and Diastereoselective Synthesis of 1,3-Oxazolidines Featuring a PalladiumCatalyzed Cyclization Reaction of 2-Butene-1,4-Diol Derivatives and Imines. Tetrahedron Lett. 2010, 51, 5131-5133.

(2) Shaghafi, M. B.; Grote, R. E.; Jarvo, E. R. Oxazolidine Synthesis by Complementary Stereospecific and Stereoconvergent Methods. Org. Lett. 2011, 13, 5188-5191.

(3) Wu, X. F.; Vovard-Le Bray, C.; Bechki, L.; Darcel, C. Iron-Catalyzed Sulfonylimine Synthesis under Neutral Conditions. Tetrahedron 2009, 65, 7380-7384.

(4) Cronin, S. A.; Gutiérrez Collar, A.; Gundala, S.; Cornaggia, C.; Torrente, E.; Manoni, F.; Botte, A.; Twamley, B.; Connon, S. J. The First Catalytic Asymmetric Cycloadditions of Imines with an Enolisable Anhydride. Org. Biomol. Chem. 2016, 14, 6955-6959. Zhang, R.; Zhang, Z.; Zhou, Q.; Yu, L.; Wang, J. The Generation of Difluoroketenimine and Its Application in the Synthesis of $\alpha, A-D i f l u o r o-\beta$-amino Amides. Angew. Chem. Int. Ed 2019, 131, 5800-5804.

(6) Uraguchi, D.; Kinoshita, N.; Kizu, T.; Ooi, T. Synergistic Catalysis of Ionic Brønsted Acid and Photosensitizer for a Redox Neutral Asymmetric $\alpha$-Coupling of NArylaminomethanes with Aldimines. J. Am. Chem. Soc. 2015, 137, 13768-13771.

(7) Reid, B. T.; Mailyan, A. K.; Zakarian, A. Total Synthesis of (+)-Guadinomic Acid via Hydroxyl-Directed Guanidylation. J. Org. Chem. 2018, 83, 9492-9496.

(8) Johnson, D. C.; Widlanski, T. S. Cerium(III) Chloride-Mediated Reactions of Sulfonamide Dianions. J. Org. Chem. 2003, 68, 5300-5309.

(9) Wang, L.; Lear, J. M.; Rafferty, S. M.; Fosu, S. C.; Nagib, D. A. Ketyl Radical Reactivity via Atom Transfer Catalysis. Science 2018, 362, 225-229.

(10) Santiago, J. V.; Burtoloso, A. C. B. Rapid Synthesis of Bicyclic N-Heterocyclic Cores from N-Terminal $\alpha, \beta$-Unsaturated Diazoketones. European J. Org. Chem. 2018, 2018, 2822-2830.

(11) Gao, D. W.; Vinogradova, E. V.; Nimmagadda, S. K.; Medina, J. M.; Xiao, Y.; Suciu, R. M.; Cravatt, B. F.; Engle, K. M. Direct Access to Versatile Electrophiles via Catalytic Oxidative Cyanation of Alkenes. J. Am. Chem. Soc. 2018, 140, 8069-8073.

(12) Han, Z.; Krishnamurthy, D.; Grover, P.; Fang, Q. K.; Senanayake, C. H. Properly Designed Modular Asymmetric Synthesis for Enantiopure Sulfinamide Auxiliaries from N-Sulfonyl-1,2,3-Oxathiazolidine-2-Oxide Agents. J. Am. Chem. Soc. 2002, 124, 78807881.

(13) Zhu, J.; Yuan, Y.; Wang, S.; Yao, Z.-J. Synthesis of 2,3-Dialkylated Tartaric Acid Esters via Visible Light Photoredox-Catalyzed Reductive Dimerization of $\alpha$-Ketoesters. ACS Omega 2017, 2, 4665-4677.

(14) Nakajima, M.; Fava, E.; Loescher, S.; Jiang, Z.; Rueping, M. Photoredox-Catalyzed Reductive Coupling of Aldehydes, Ketones, and Imines with Visible Light. Angew. Chem. Int. Ed. 2015, 54, 8828-8832.

(15) F. Chemla, V. Hebbe, J. Normant, Synthesis 2000, 1, 75. 
<smiles>CCCCC(I)OC(C)=O</smiles>

$1 a$
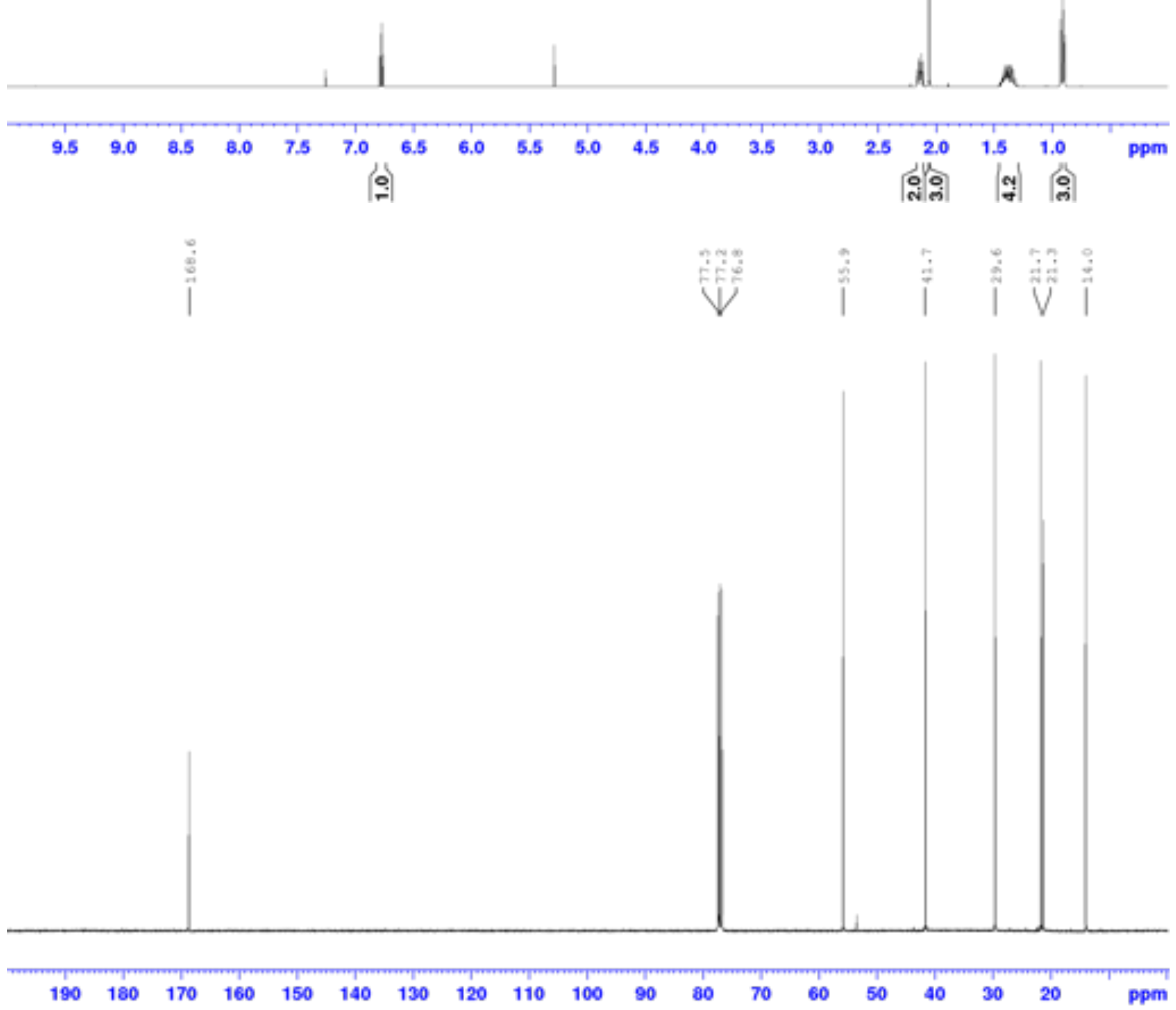


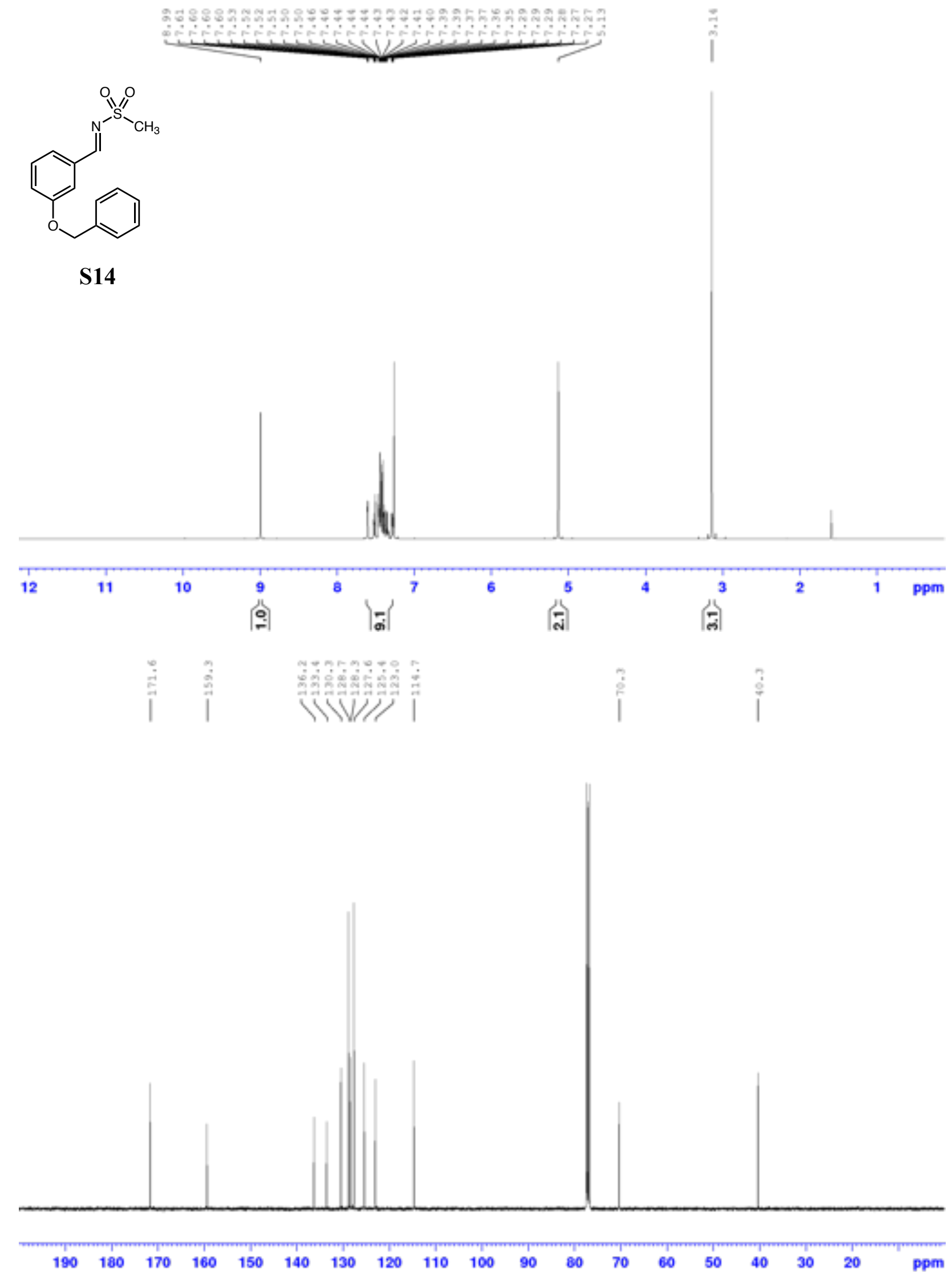



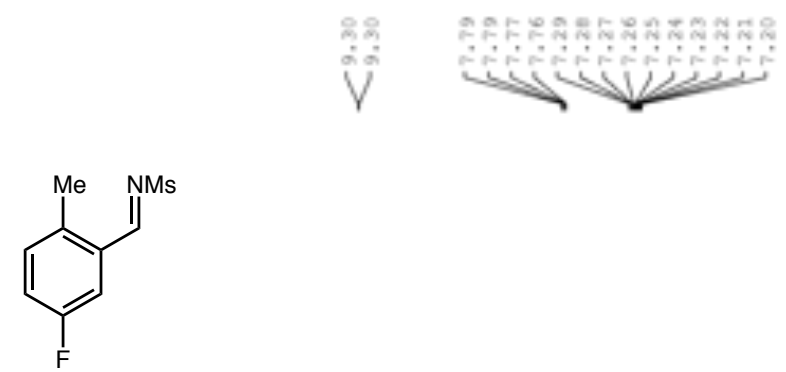

\section{S15}
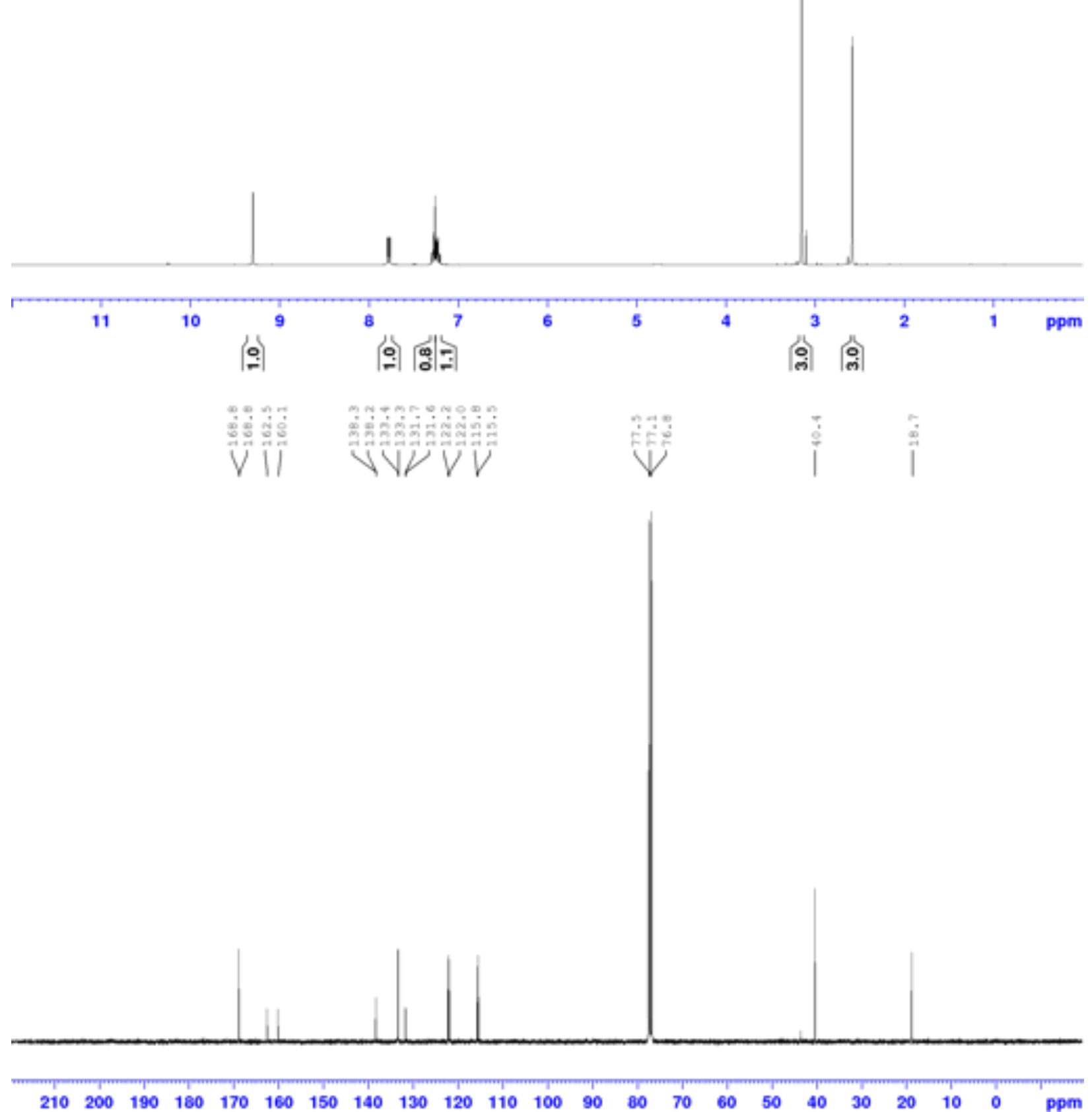


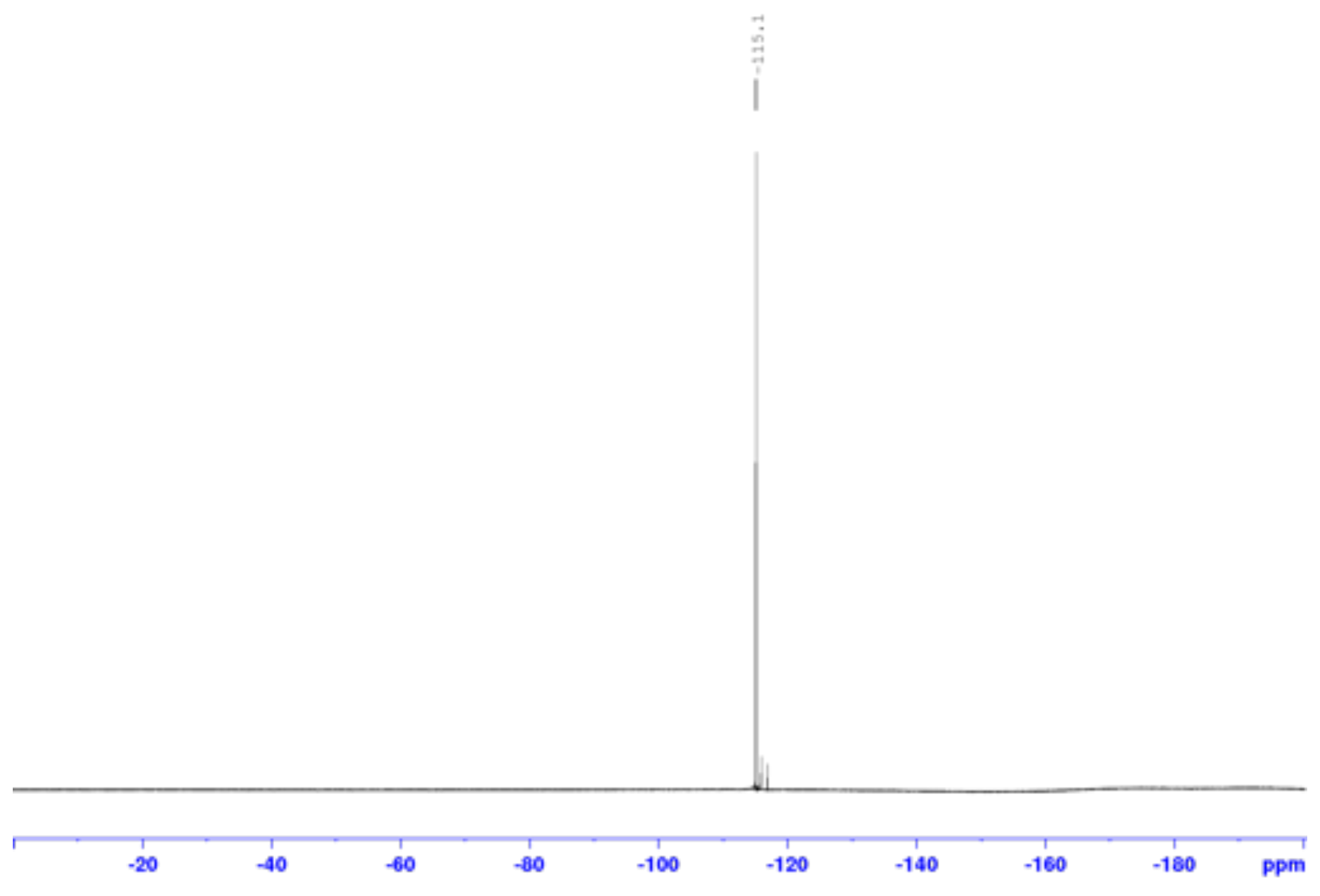




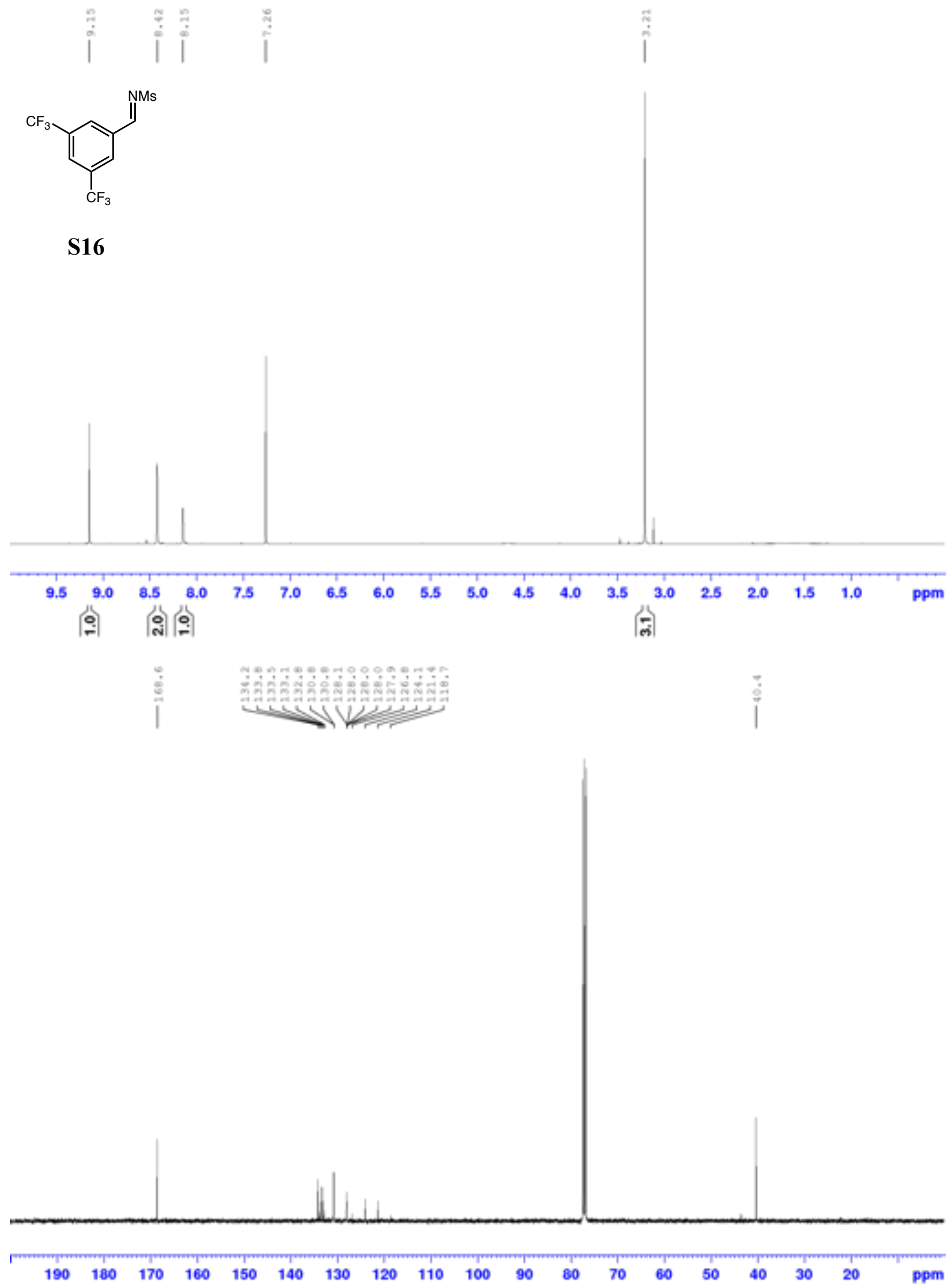




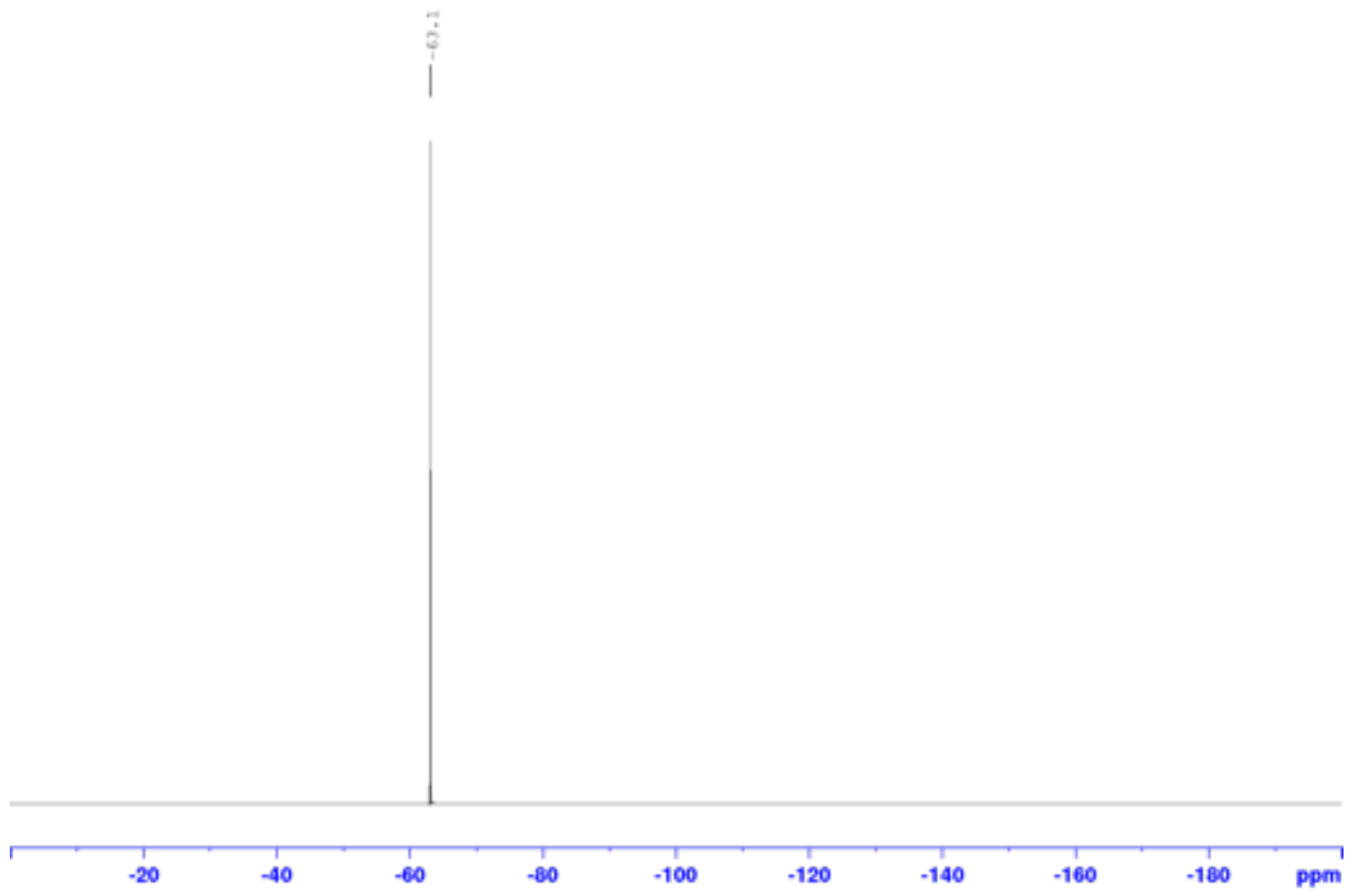




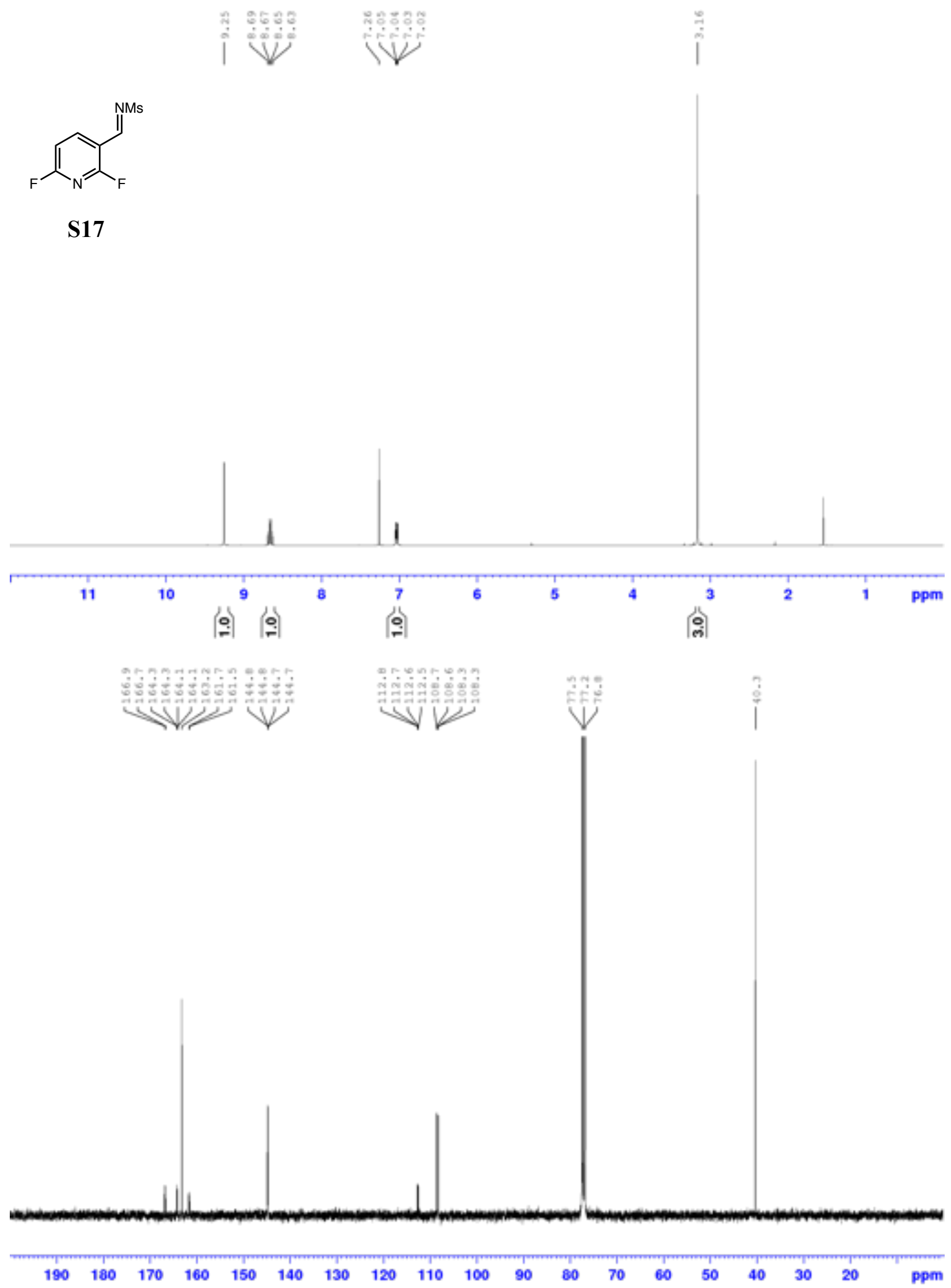




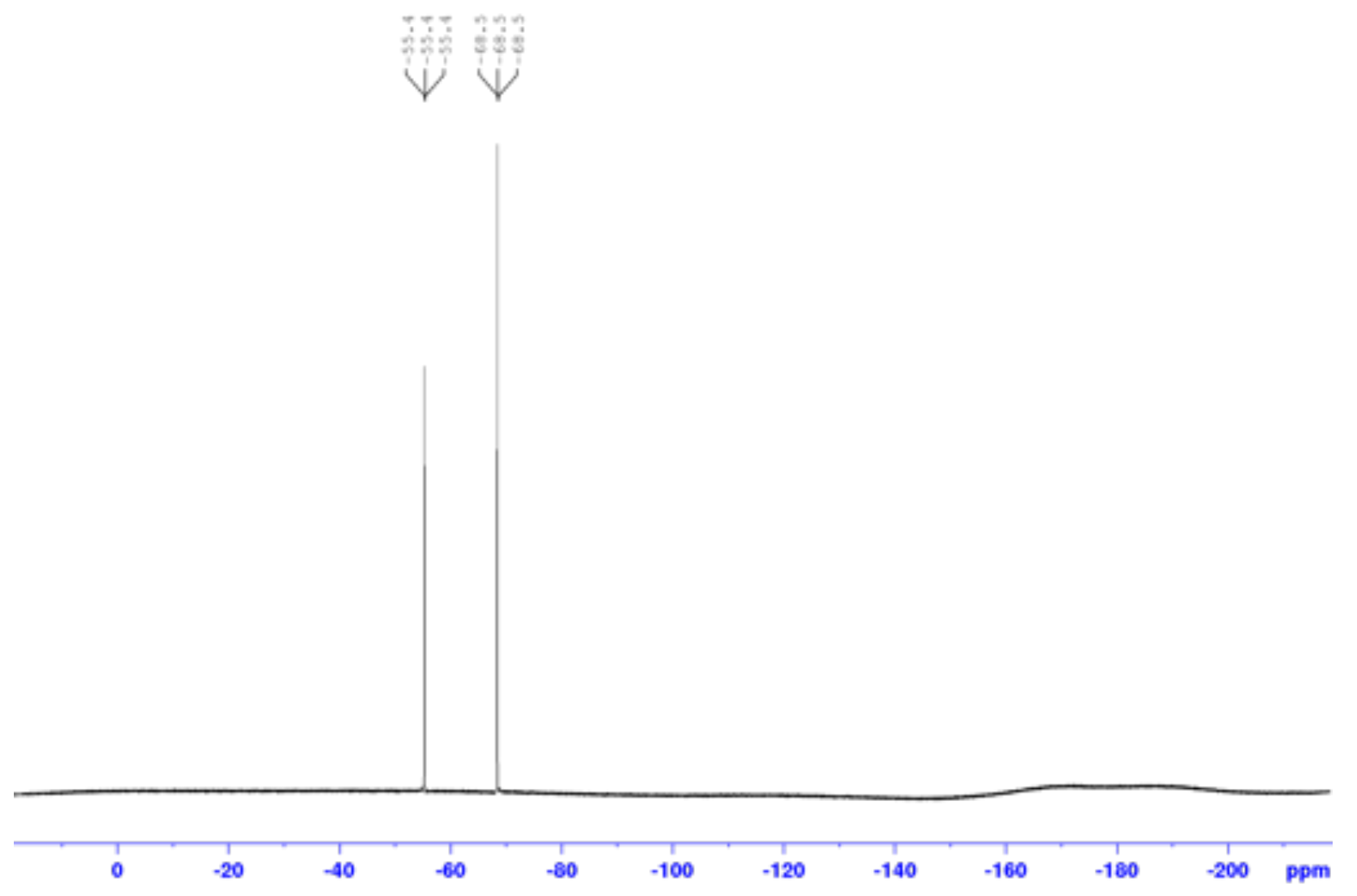




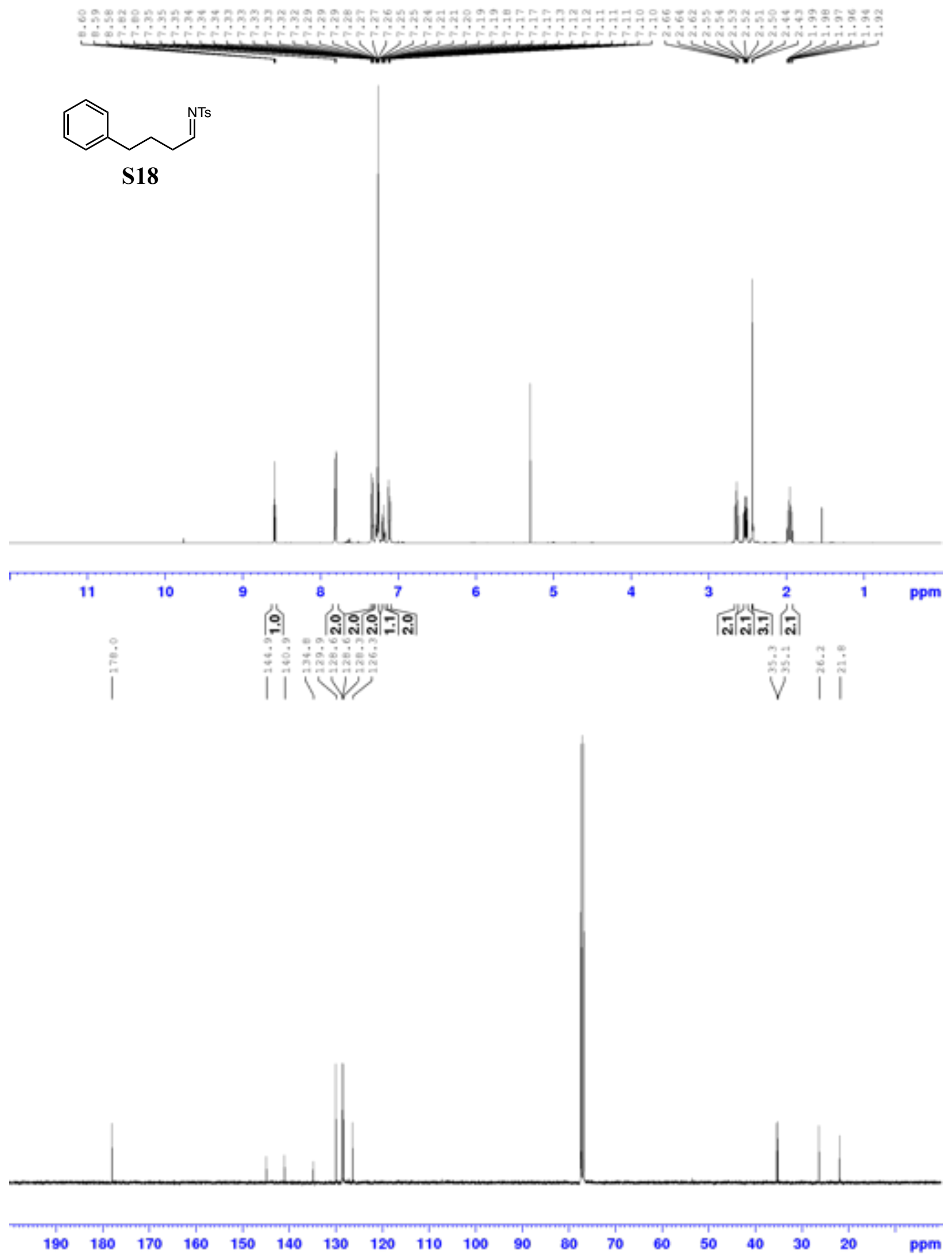




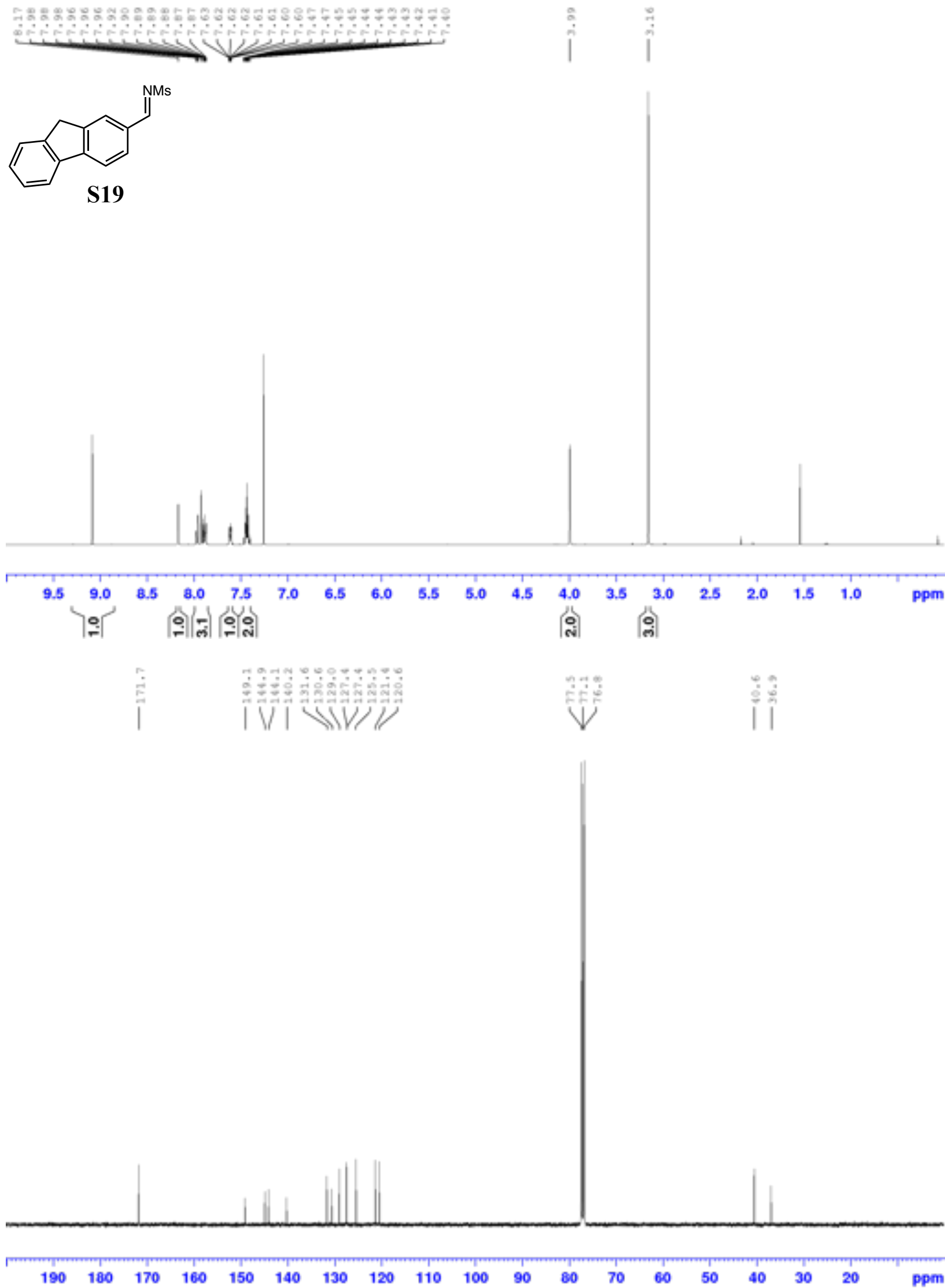



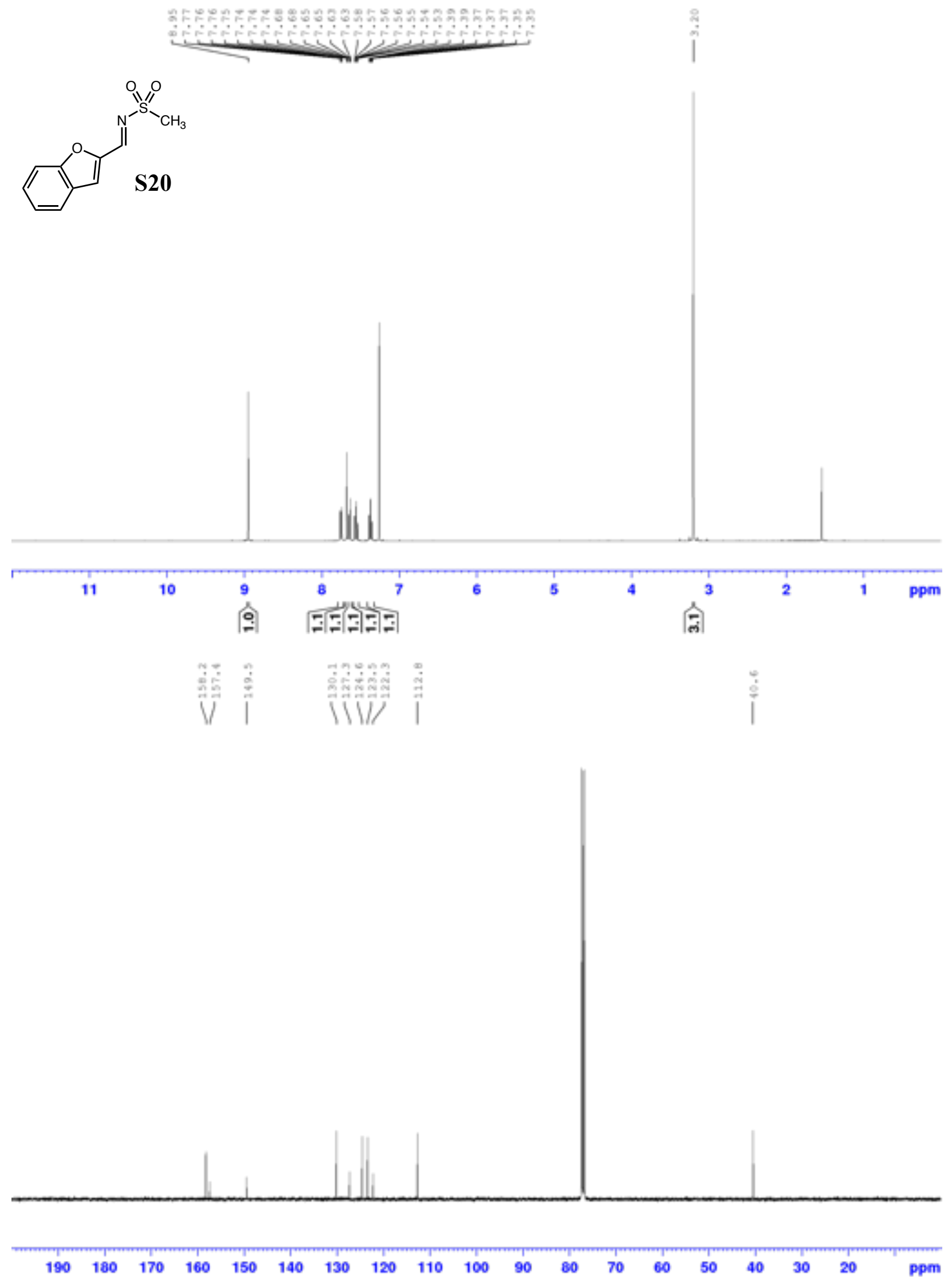


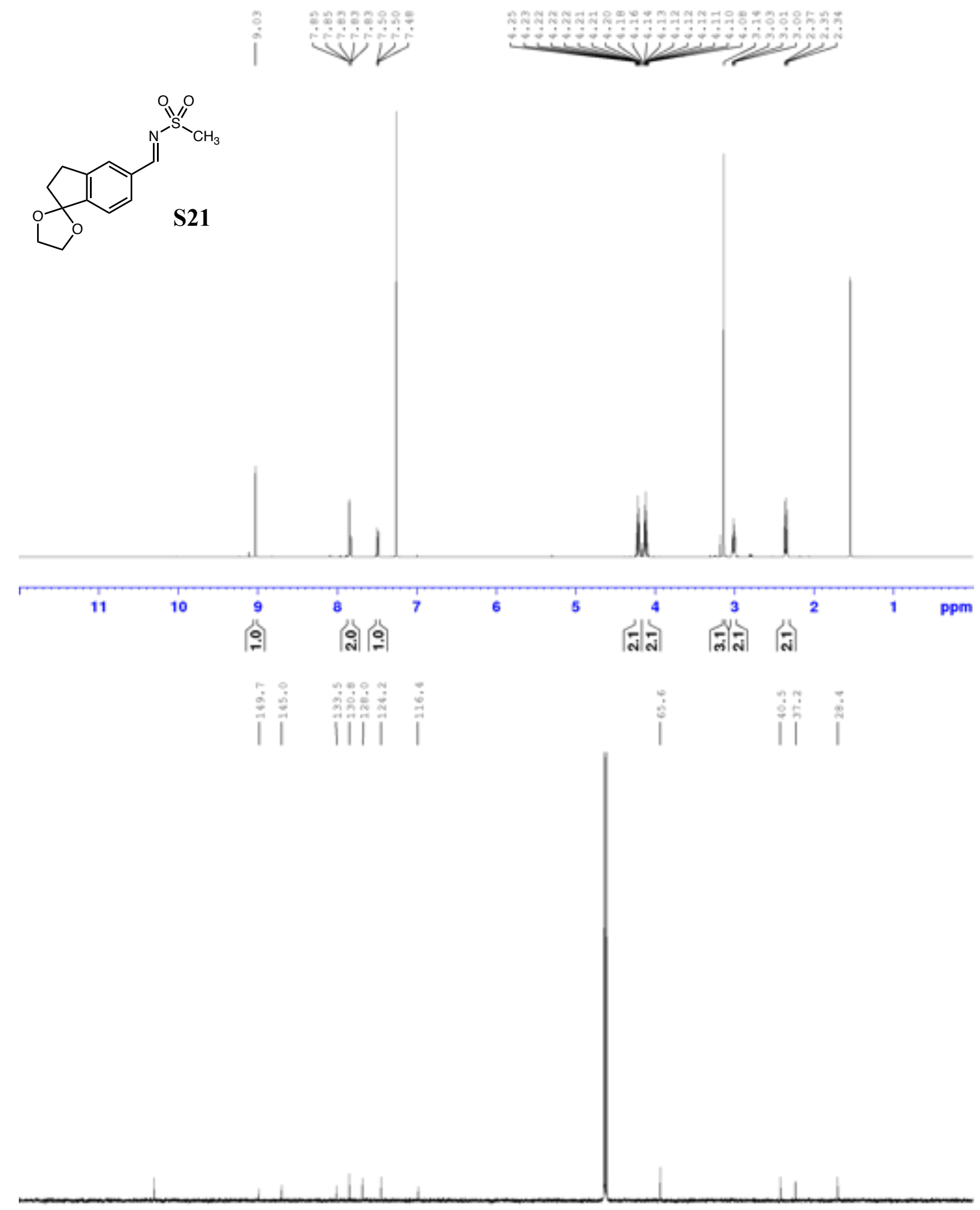

$\begin{array}{lllllllllllllllllll}190 & 180 & 170 & 160 & 150 & 140 & 130 & 120 & 110 & 100 & 90 & 80 & 70 & 60 & 50 & 40 & 30 & 20 & \mathrm{ppm}\end{array}$ 

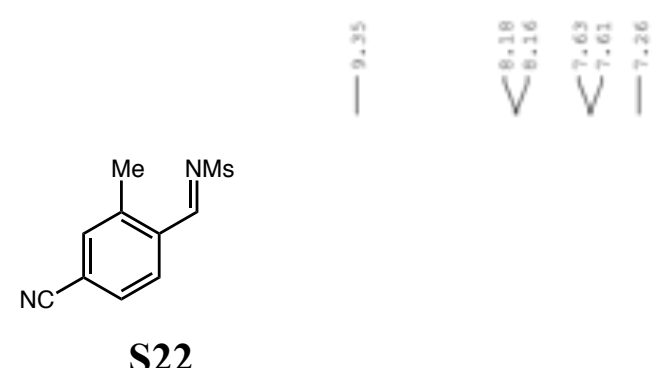

S22
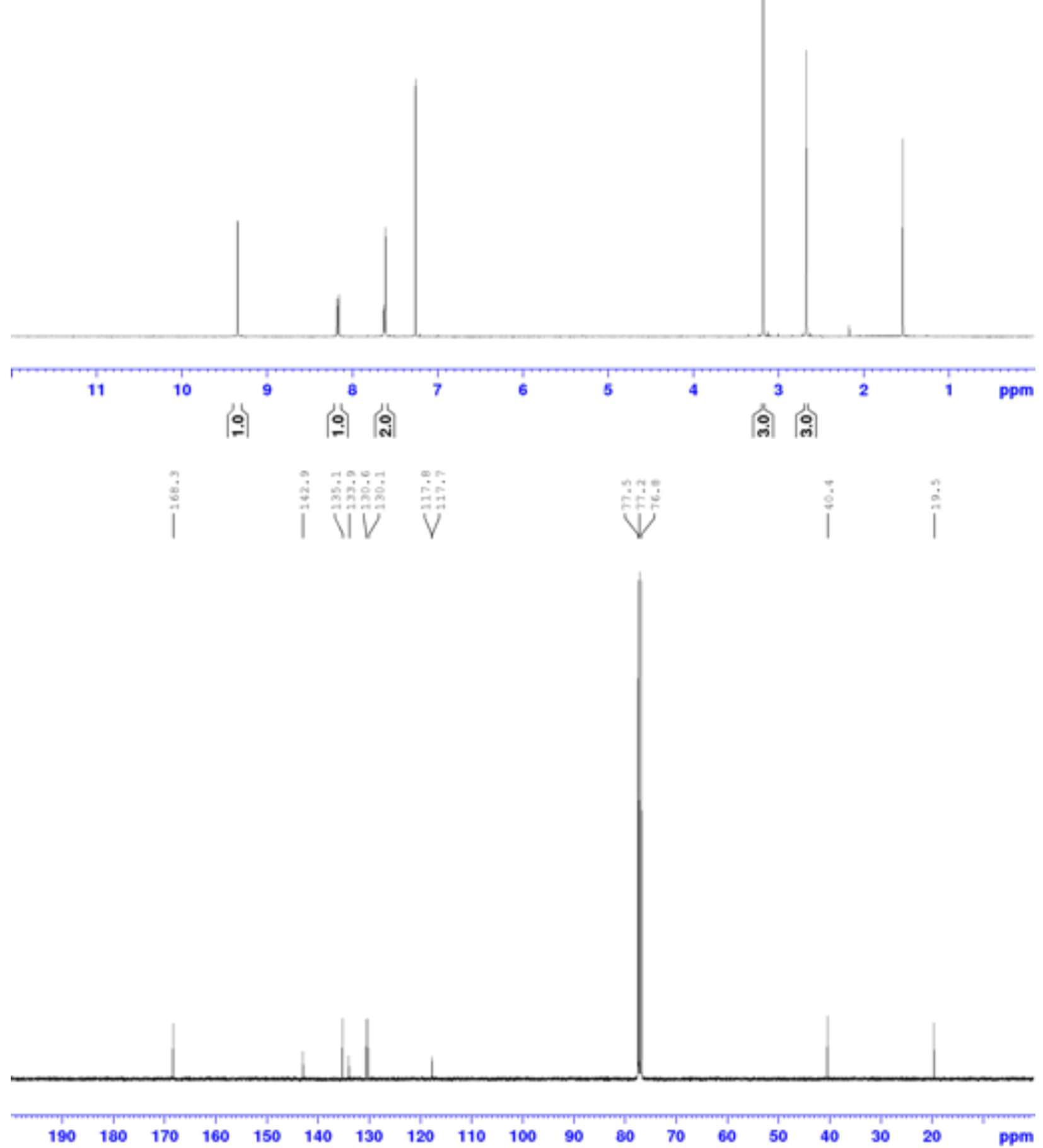


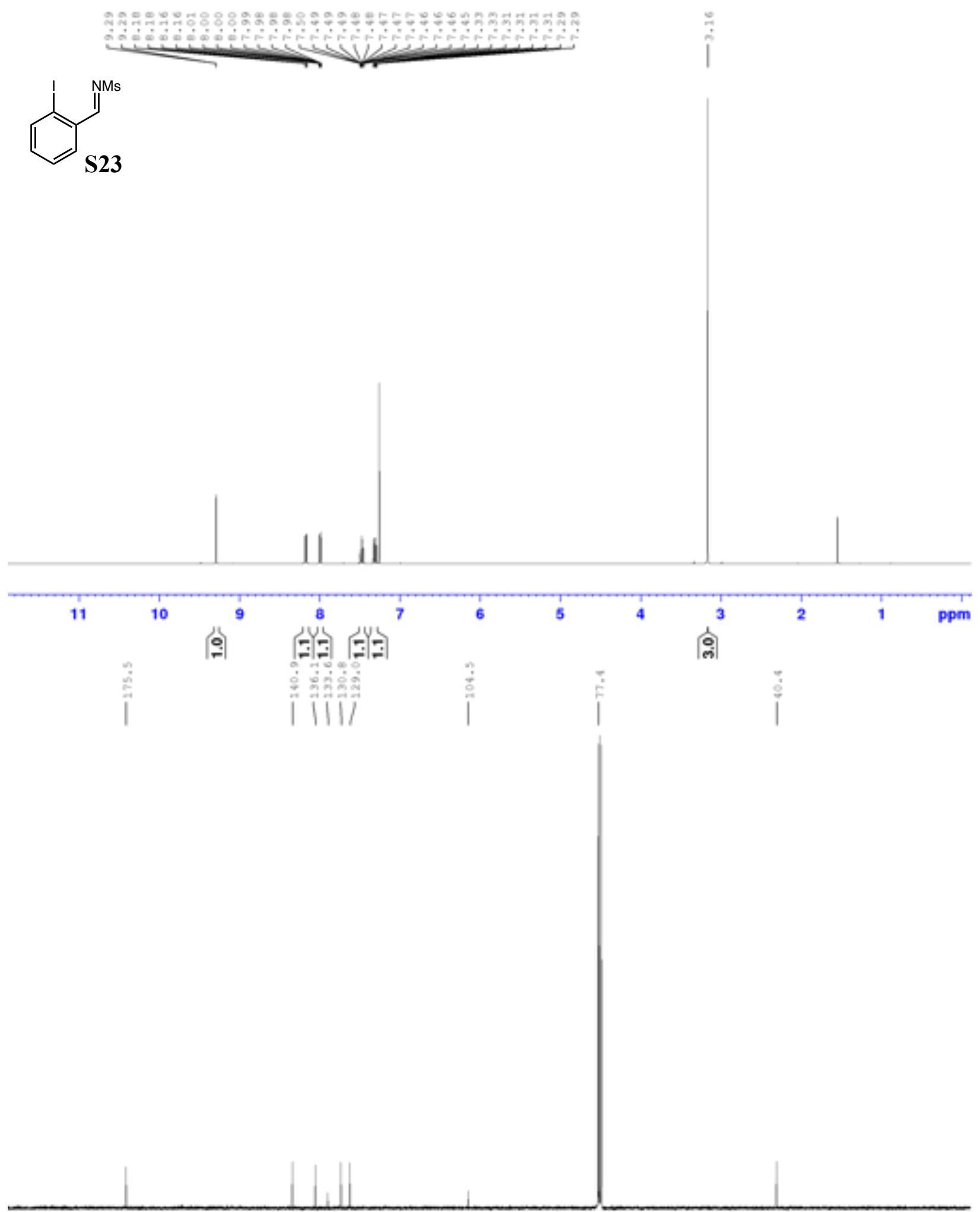

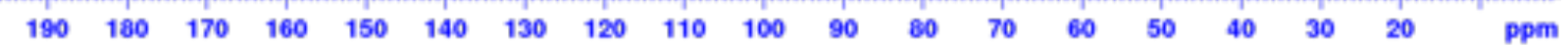




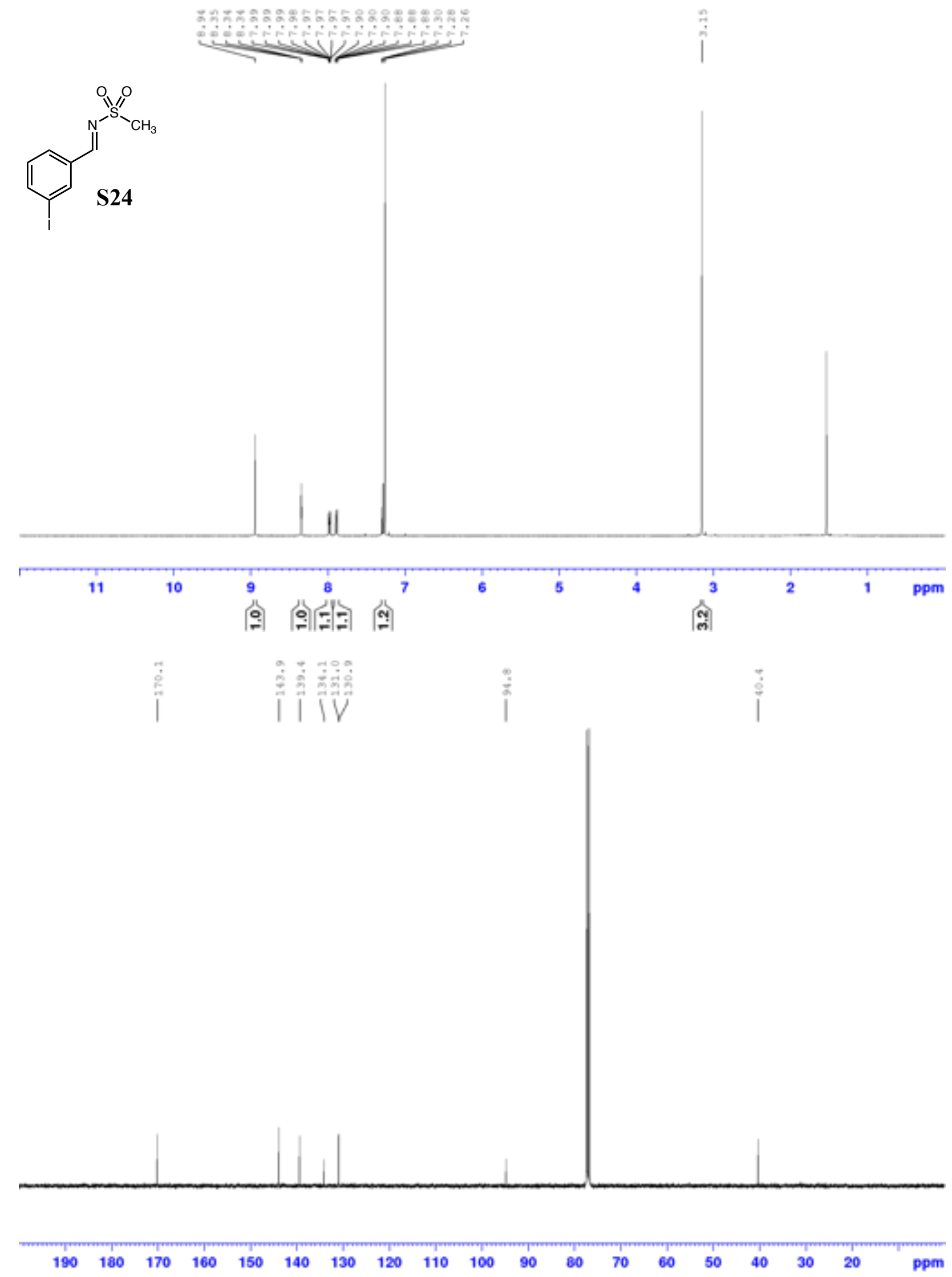



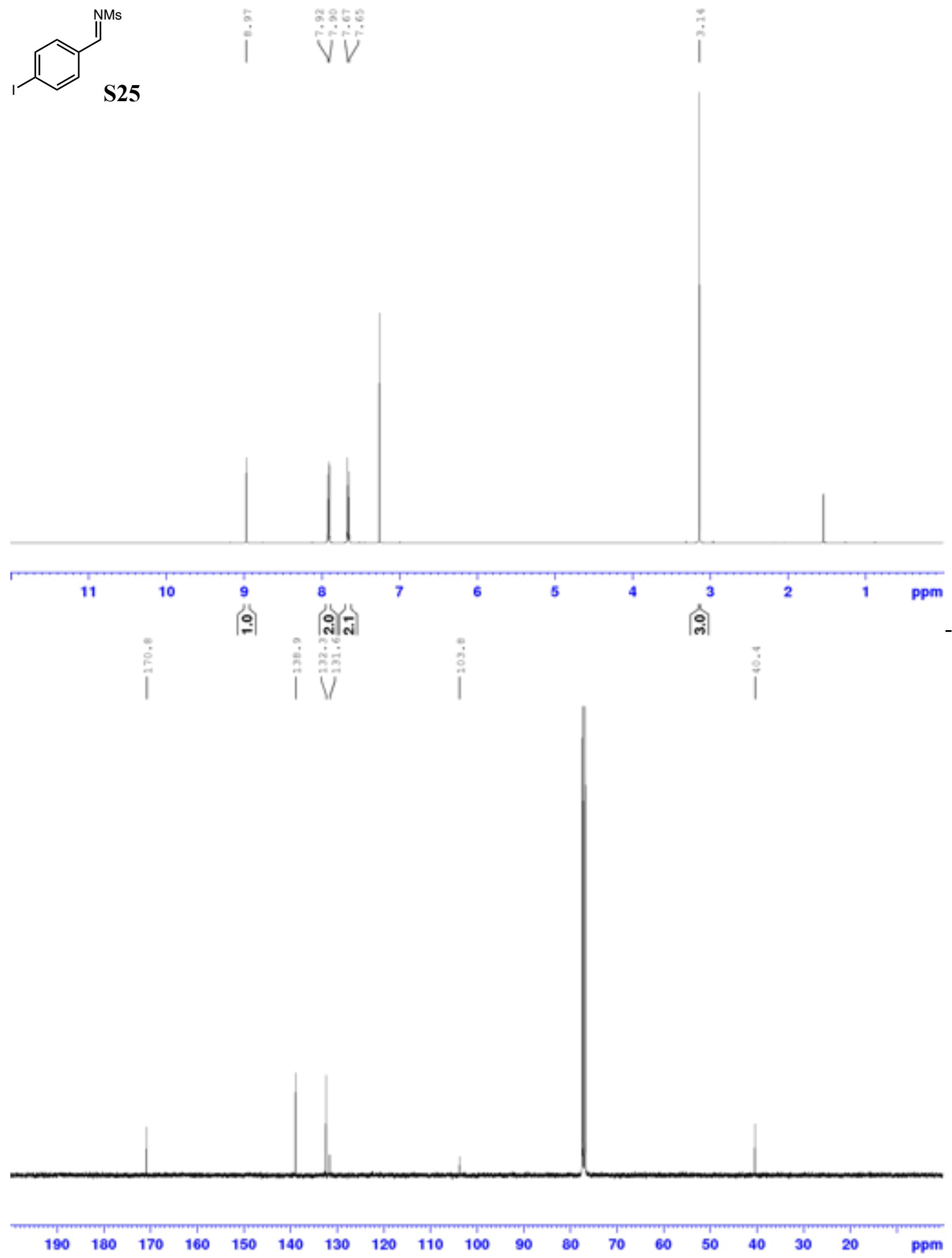


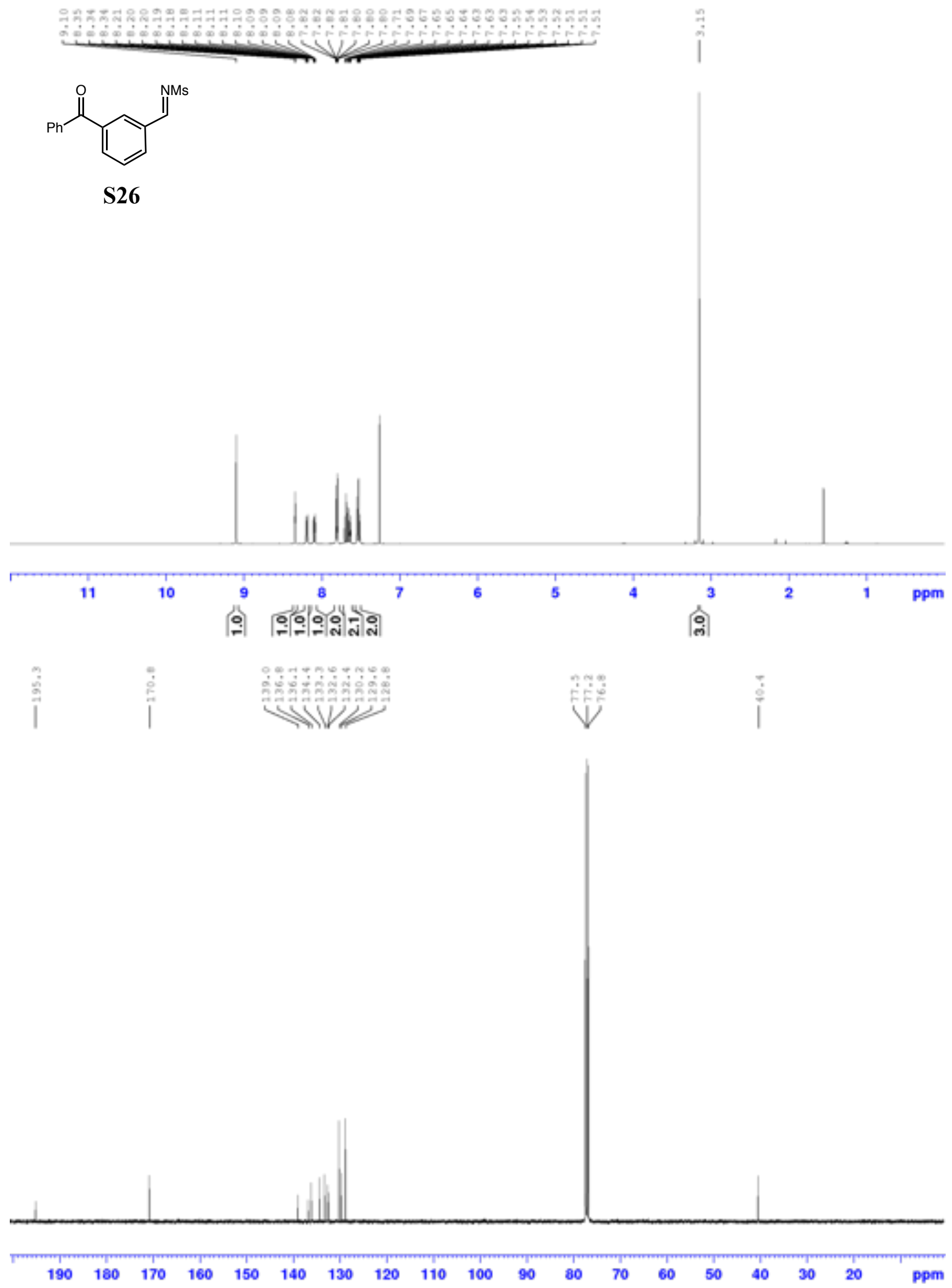



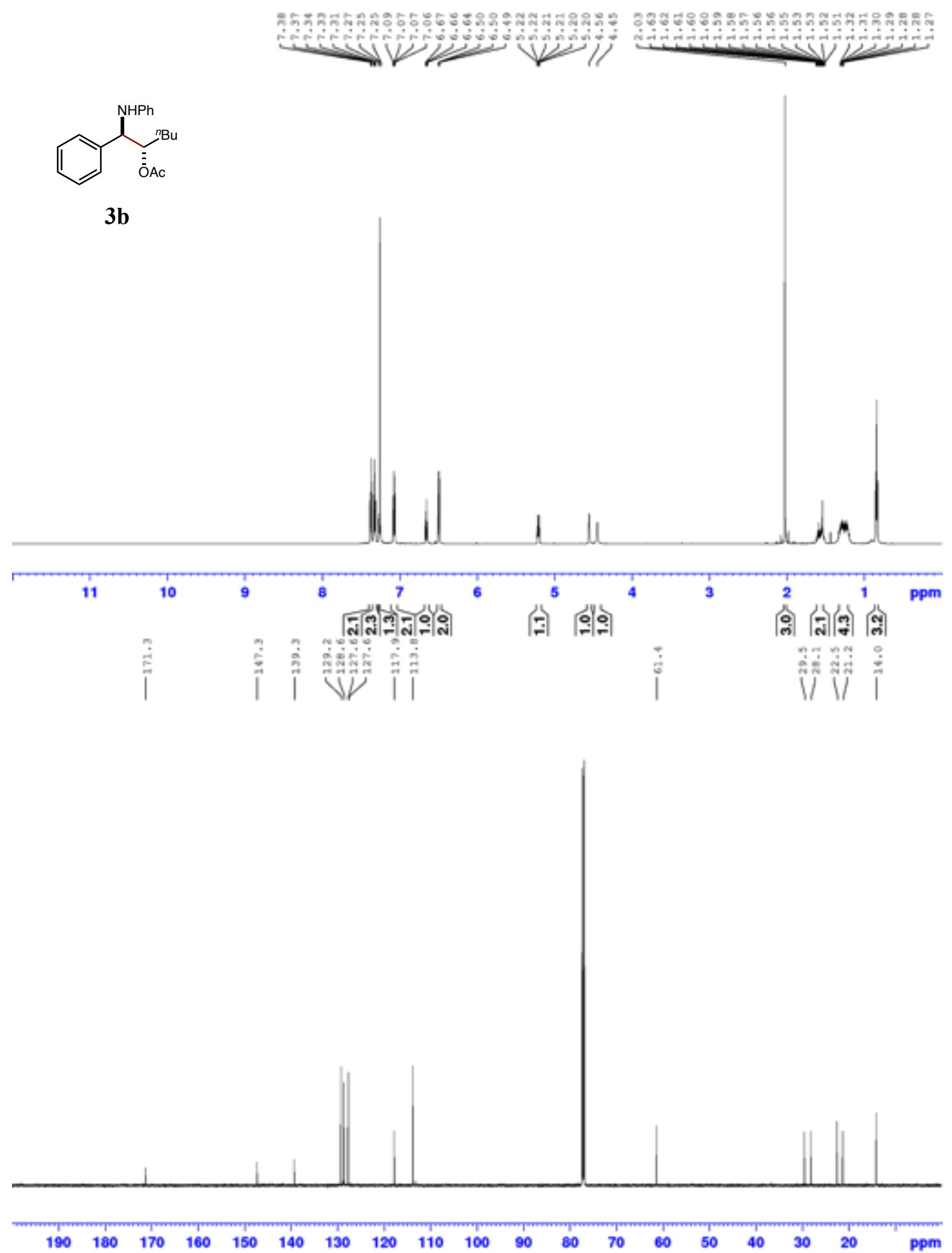
<smiles>CC(=O)OC([18O])C(c1ccccc1)[N+](=O)[O-]</smiles>

mixture of diastereomers

$$
\text { 3c }
$$
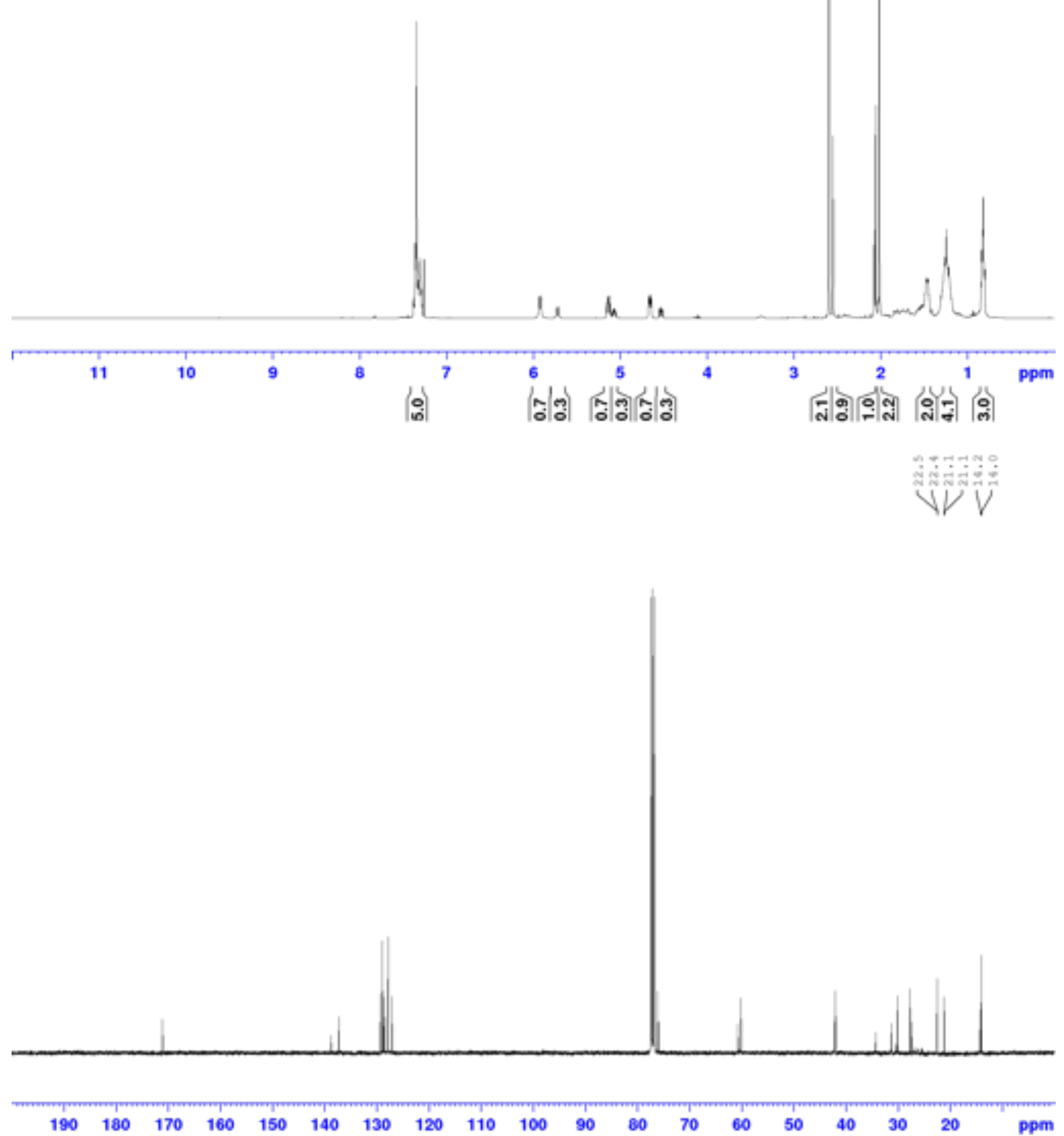

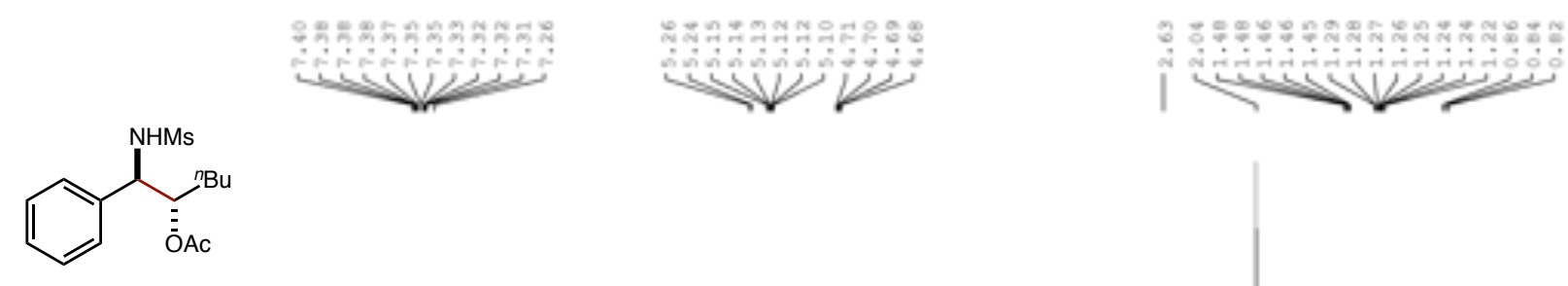

major diastereomer

$3 c$

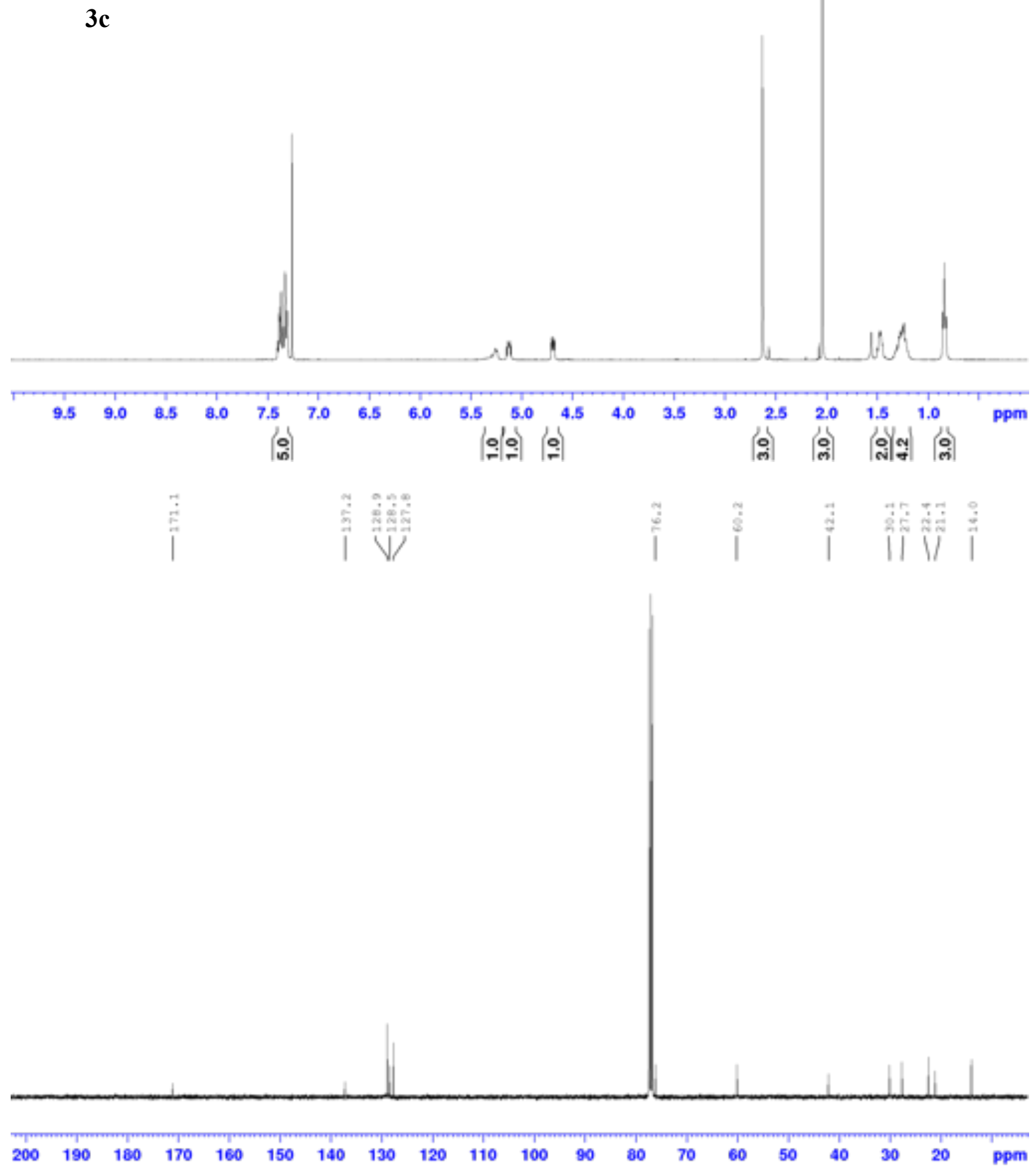




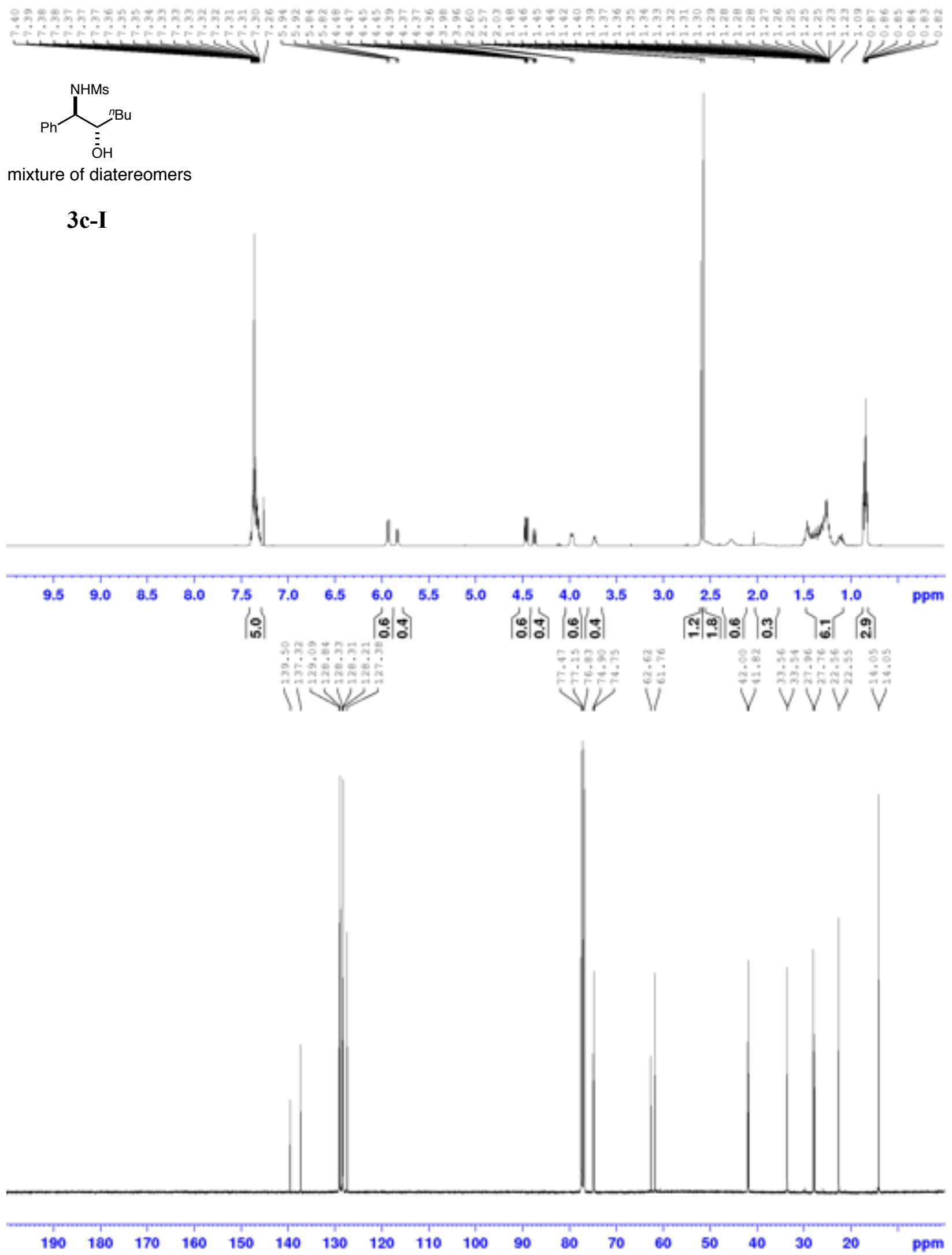


<smiles>CN1C(=O)OC([18O])C1c1ccccc1</smiles>

mixture of diastereomers

\section{3c-II}
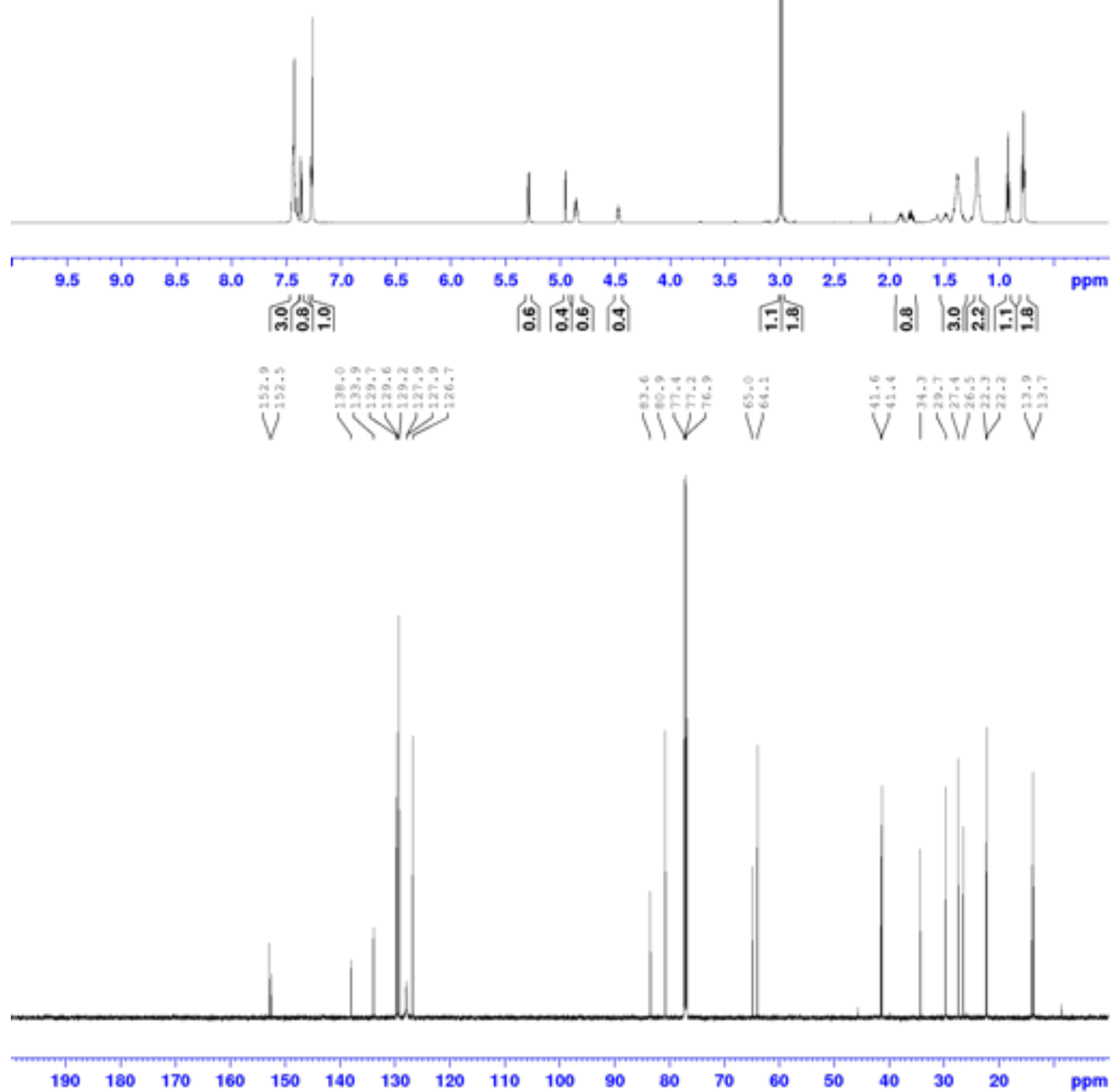


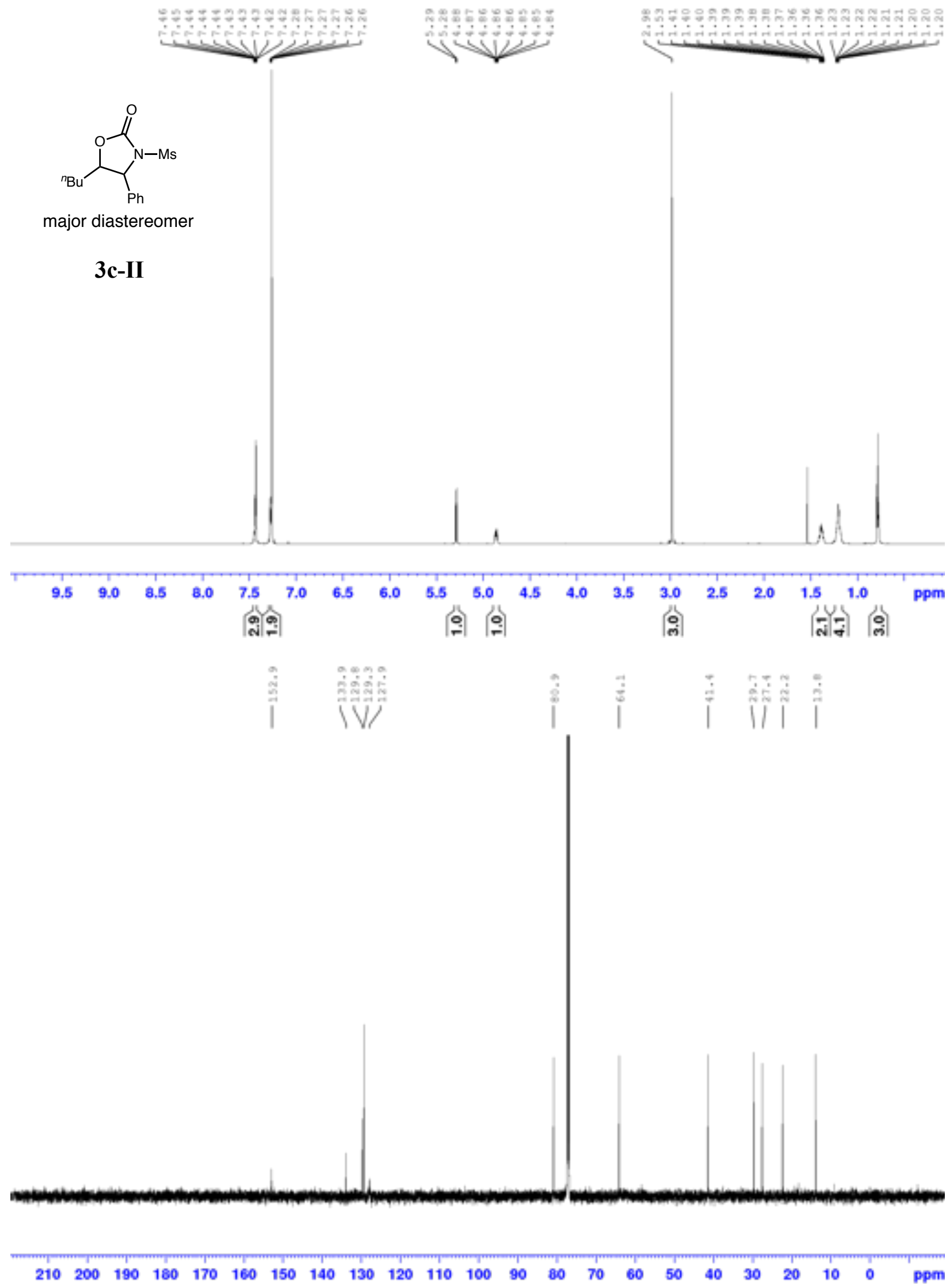




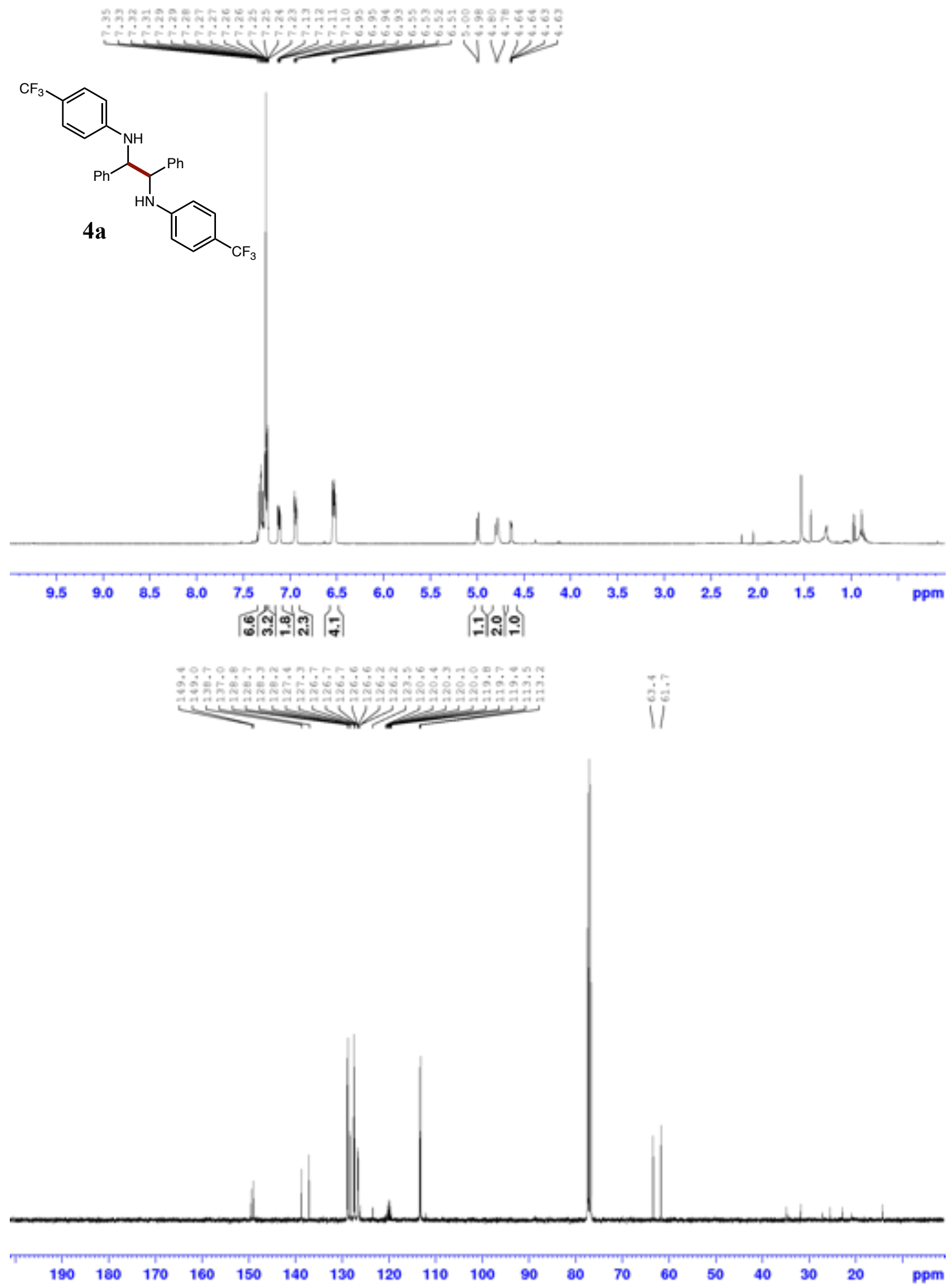




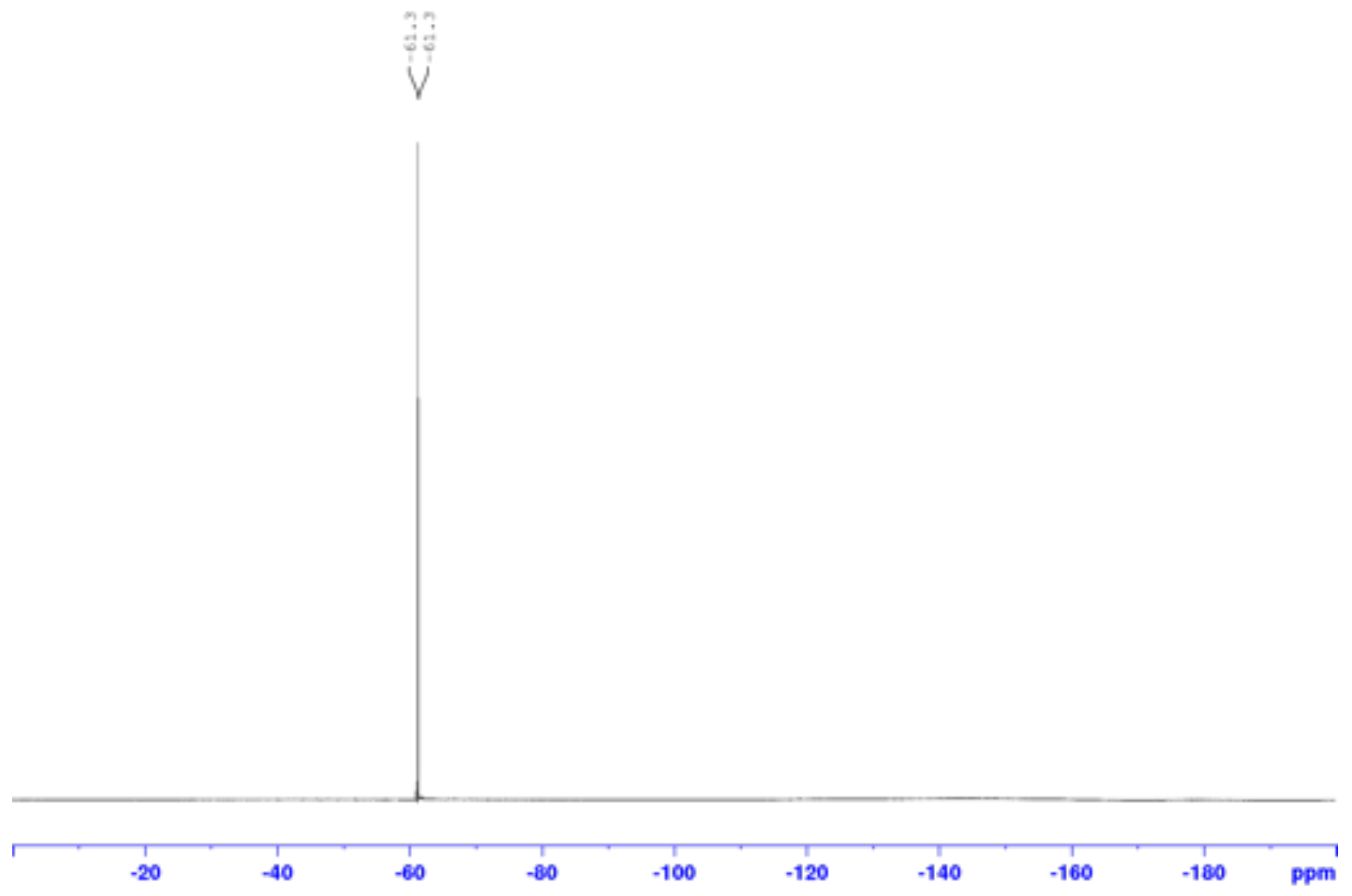



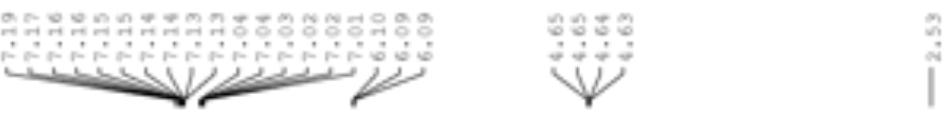

Ms

4c
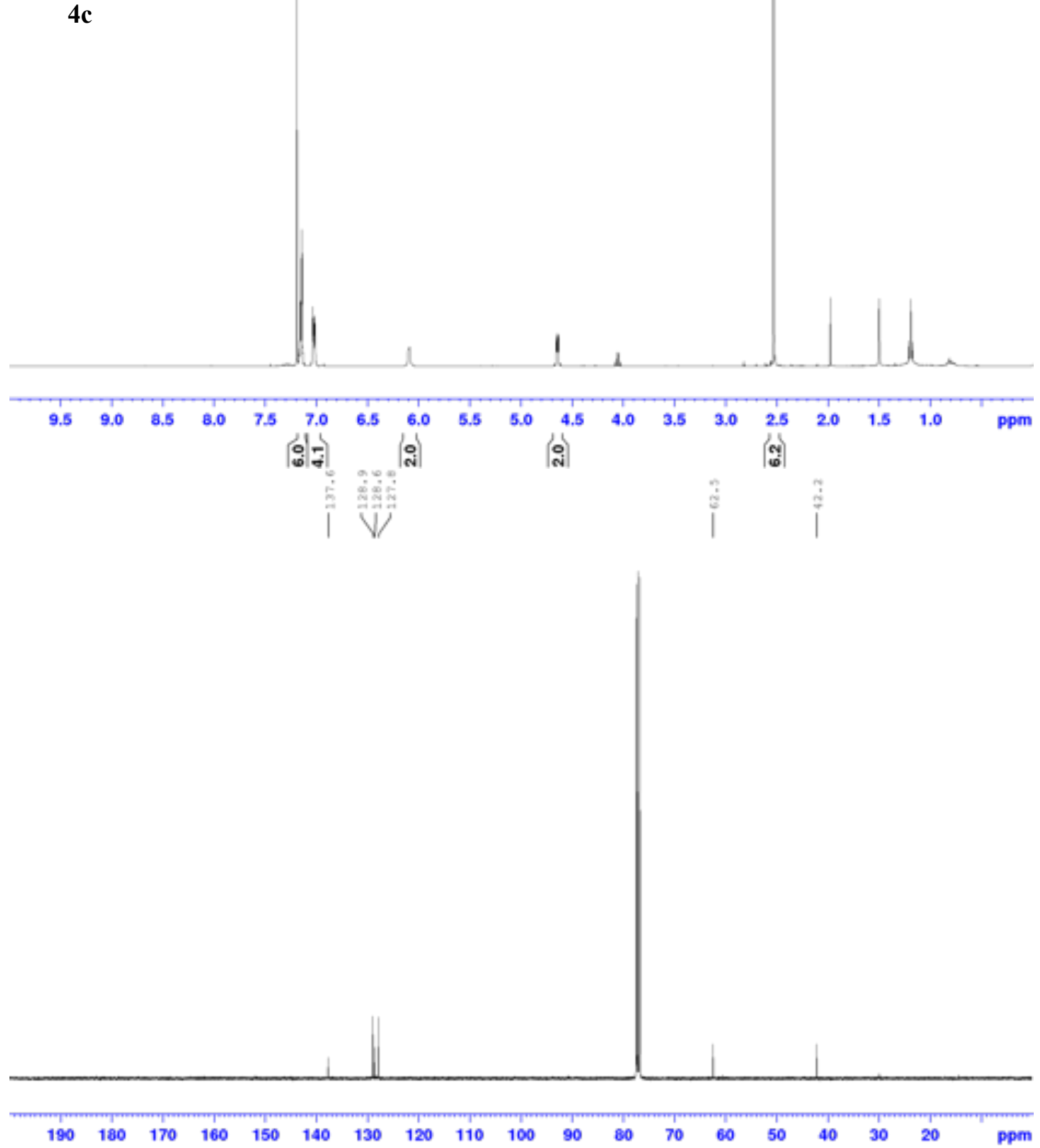

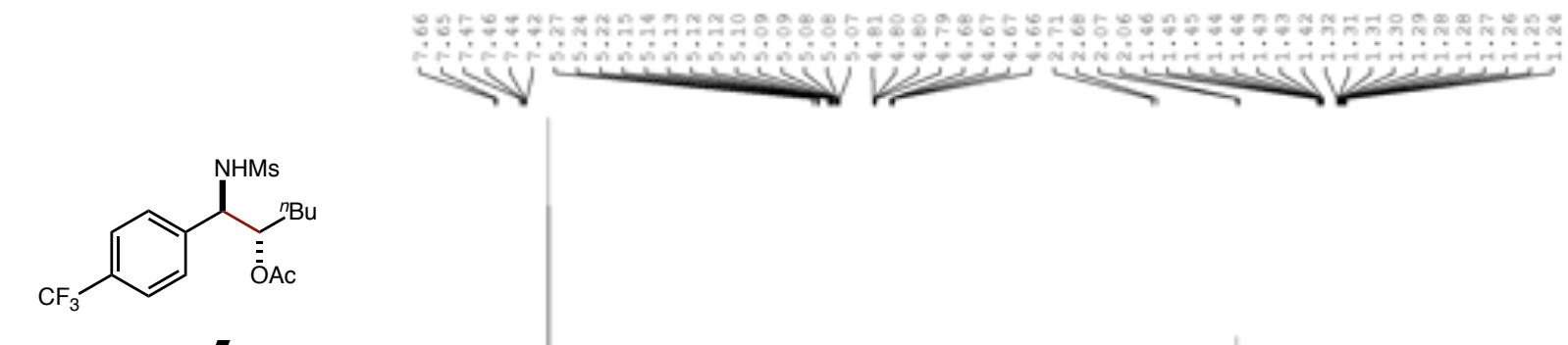

5
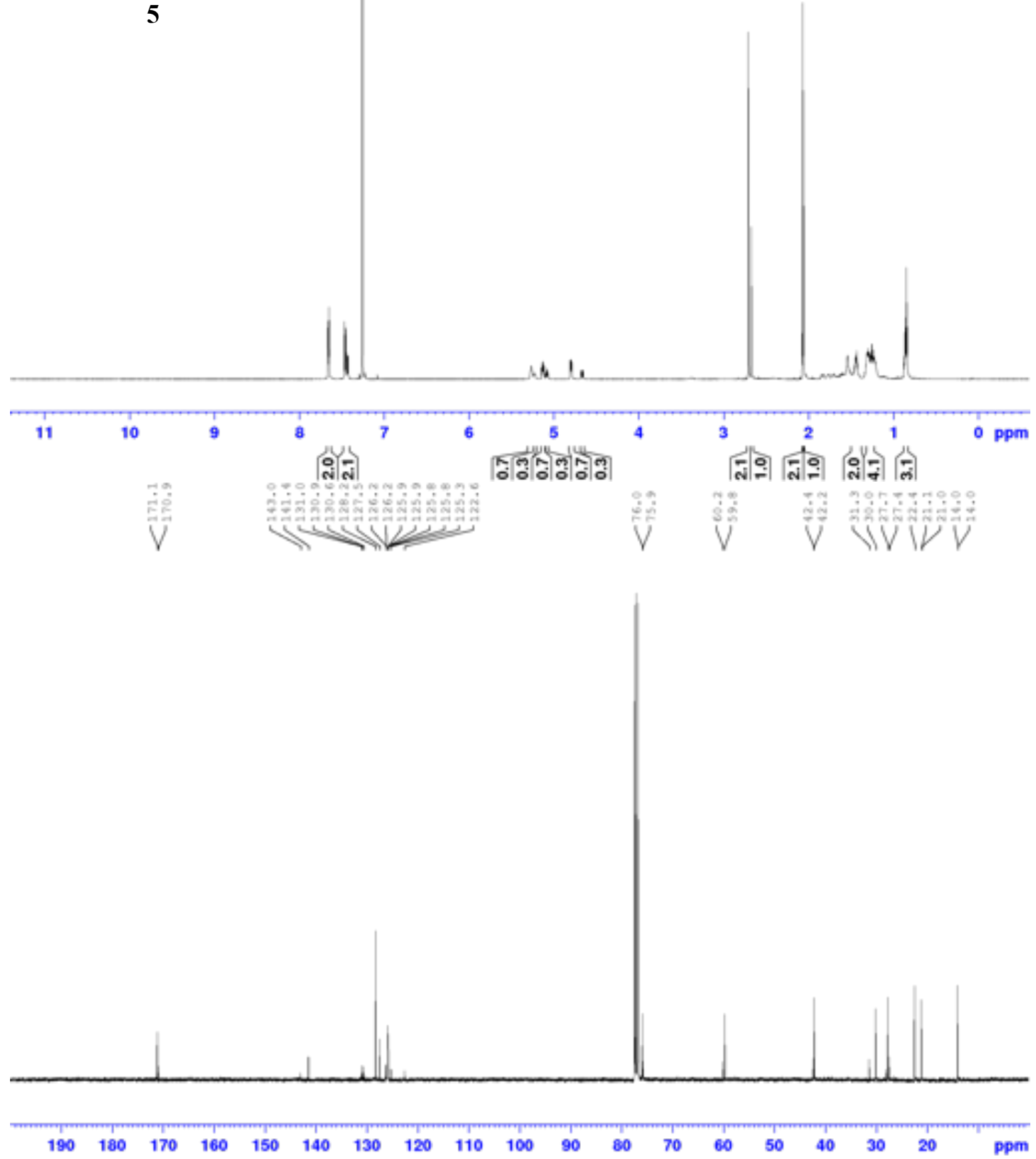


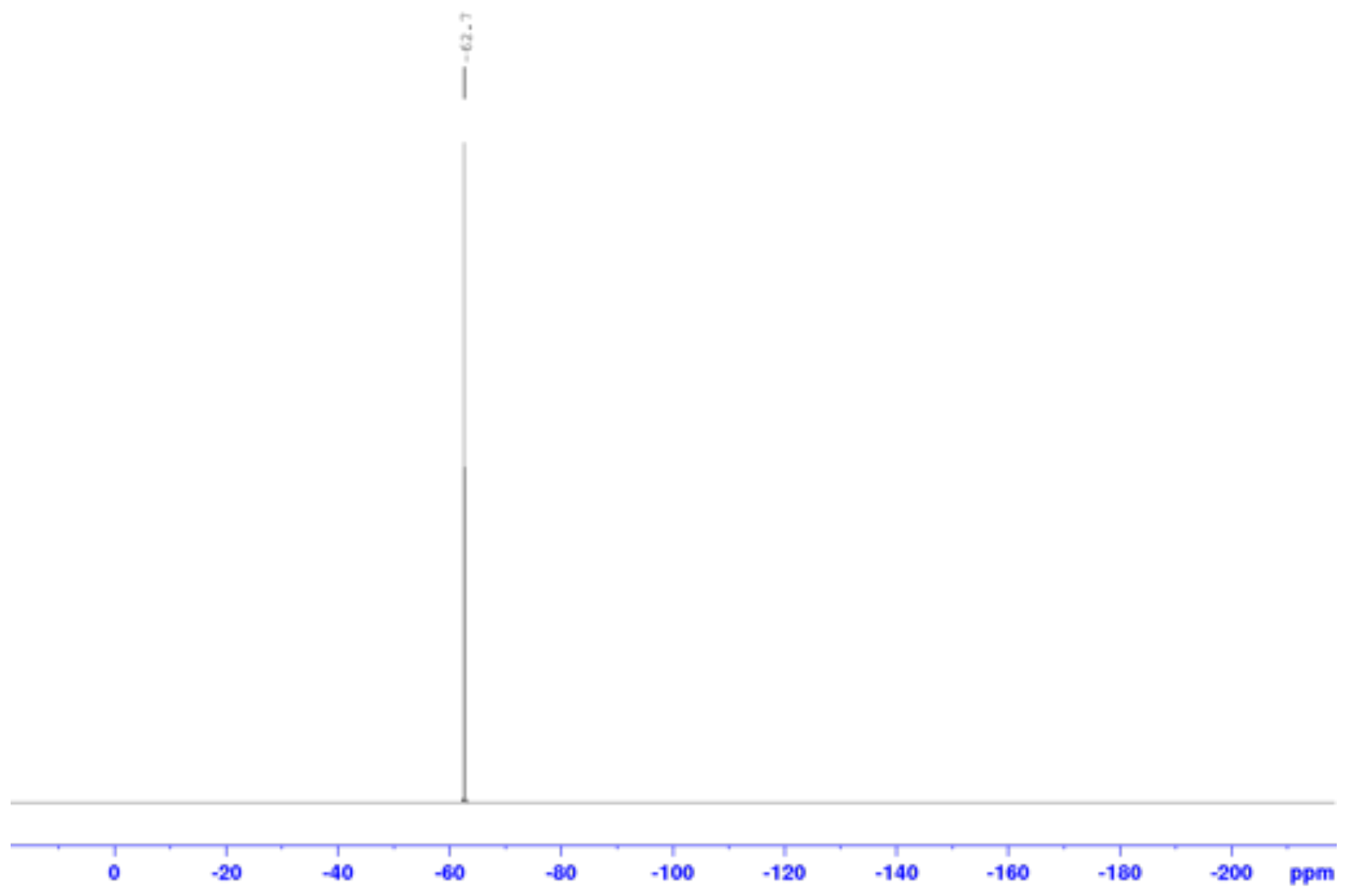




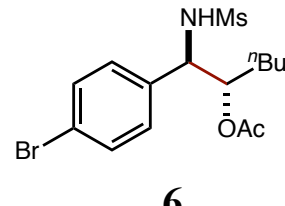

6

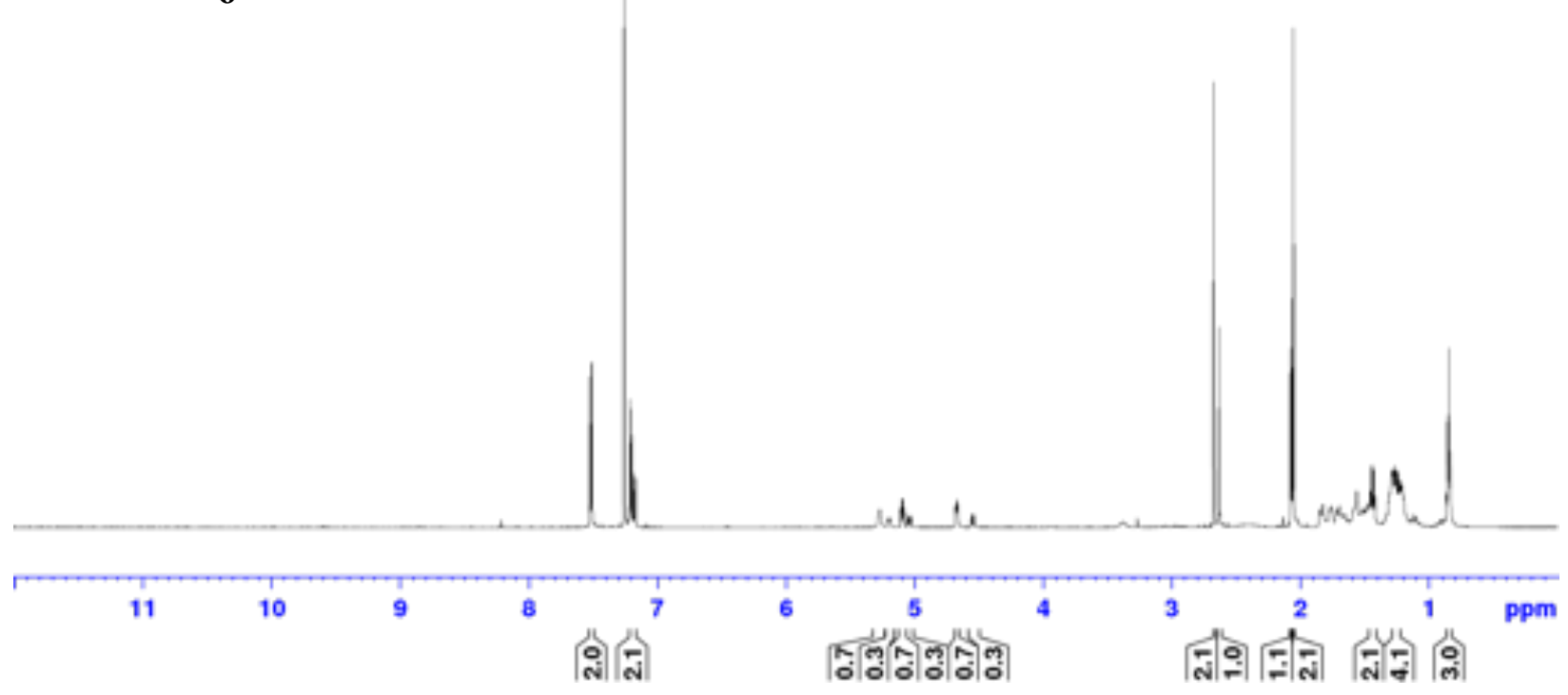

V VVVV

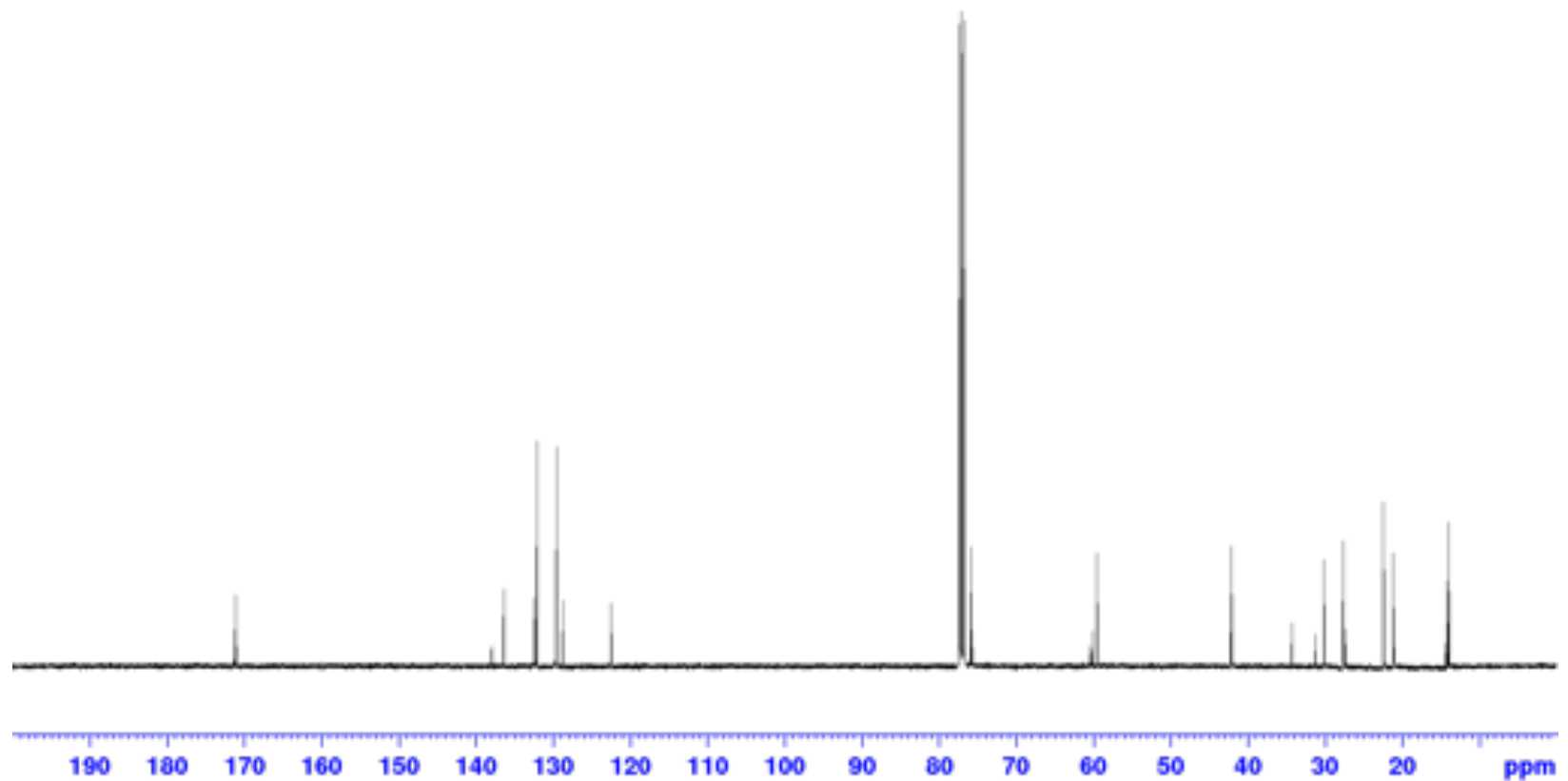




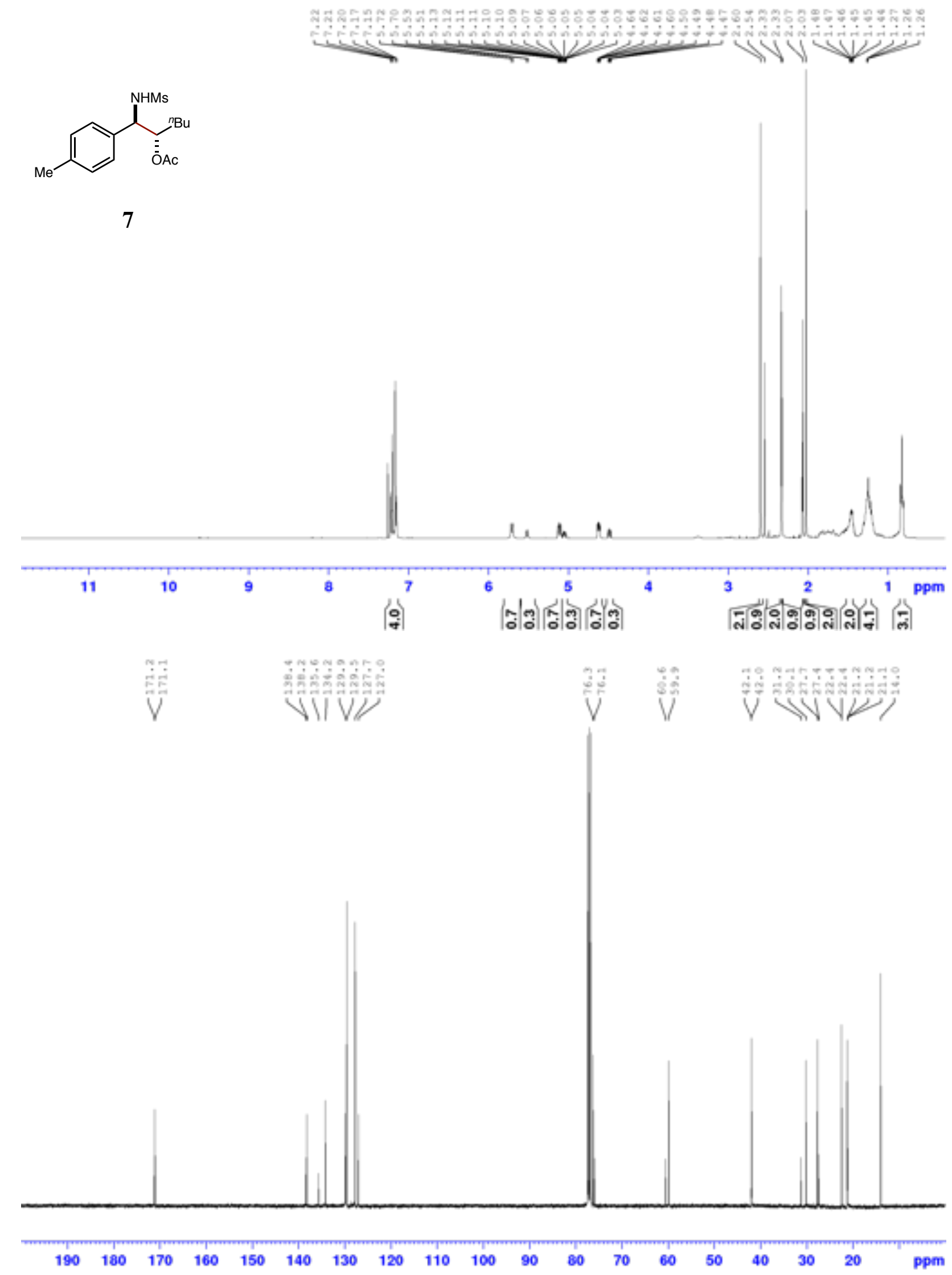


<smiles>COc1ccc(C(N)C([18OH])[18OH])cc1</smiles>

8
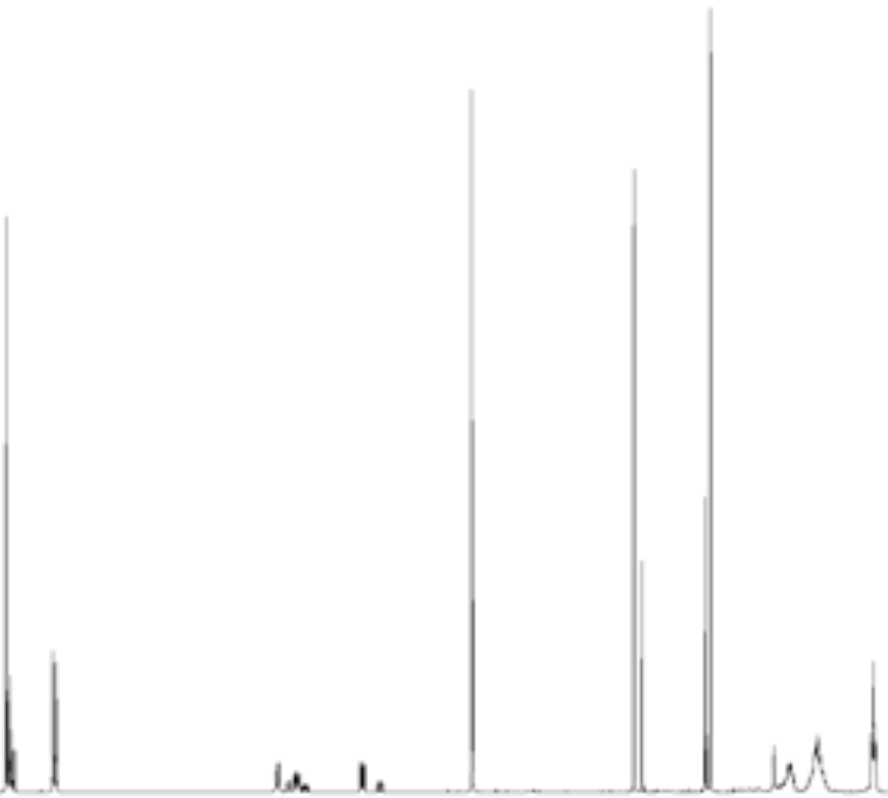

$\begin{array}{llllll}11 & 10 & 9 & 8 & 7 & 6\end{array}$

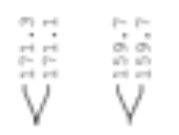
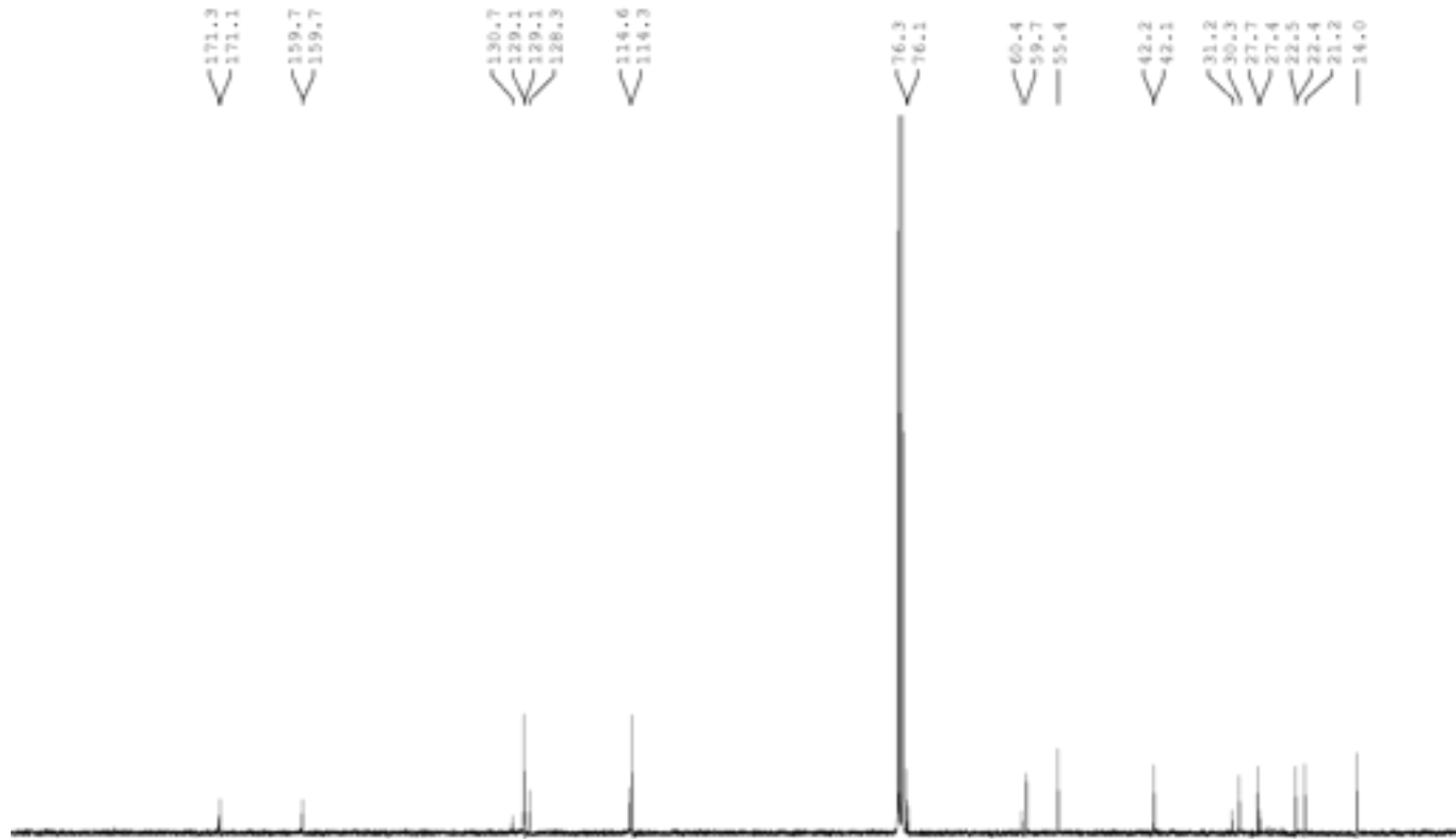


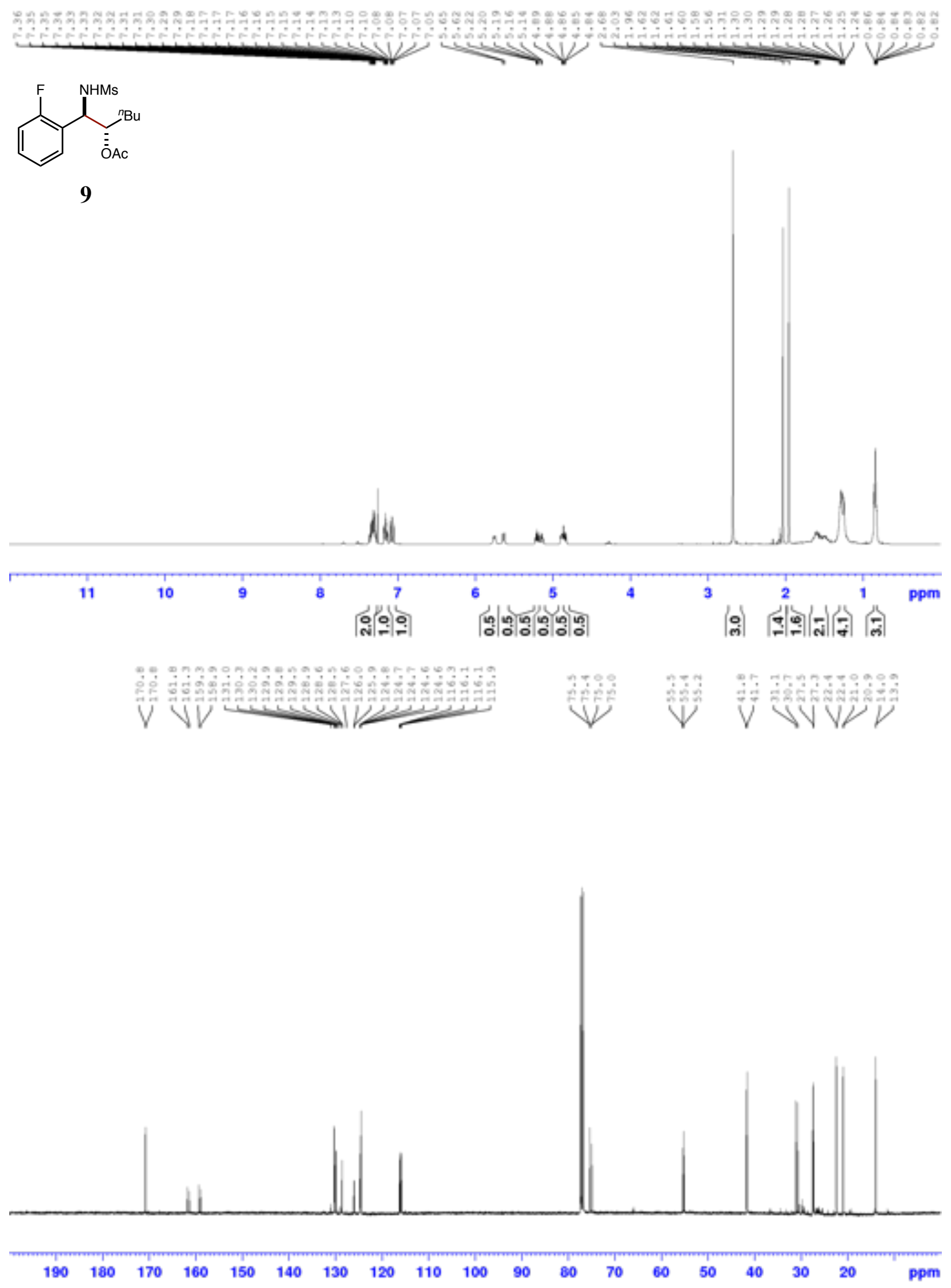




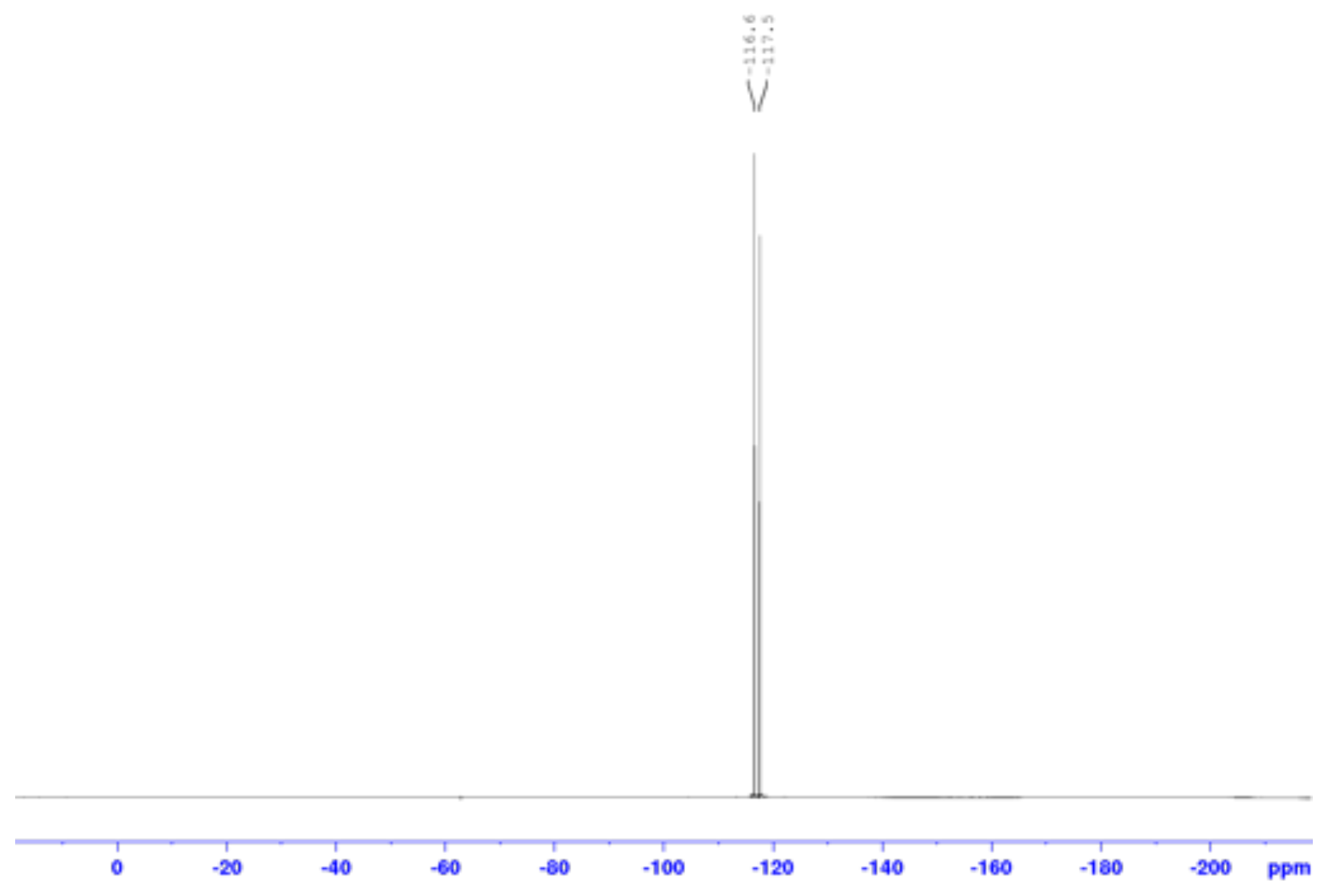



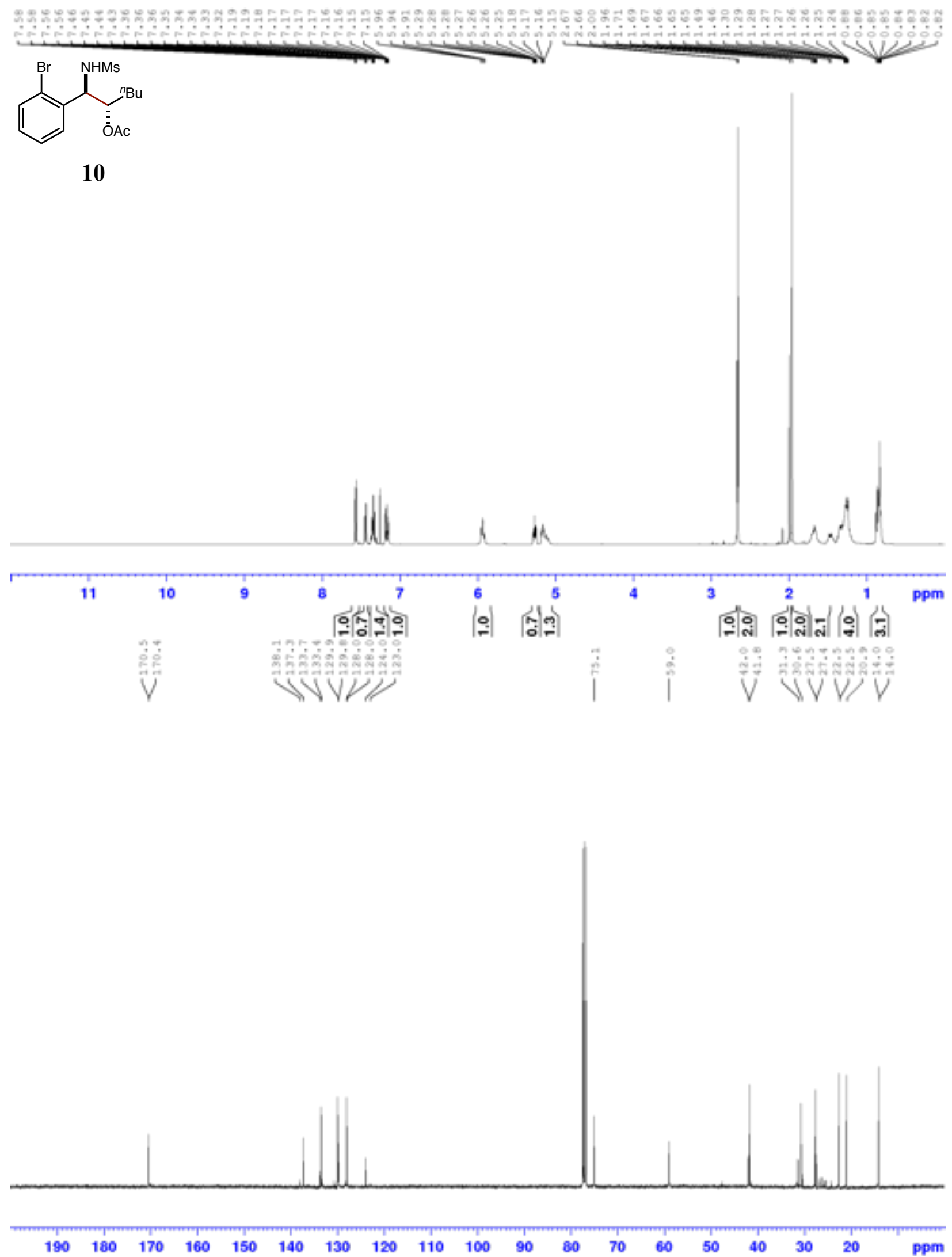


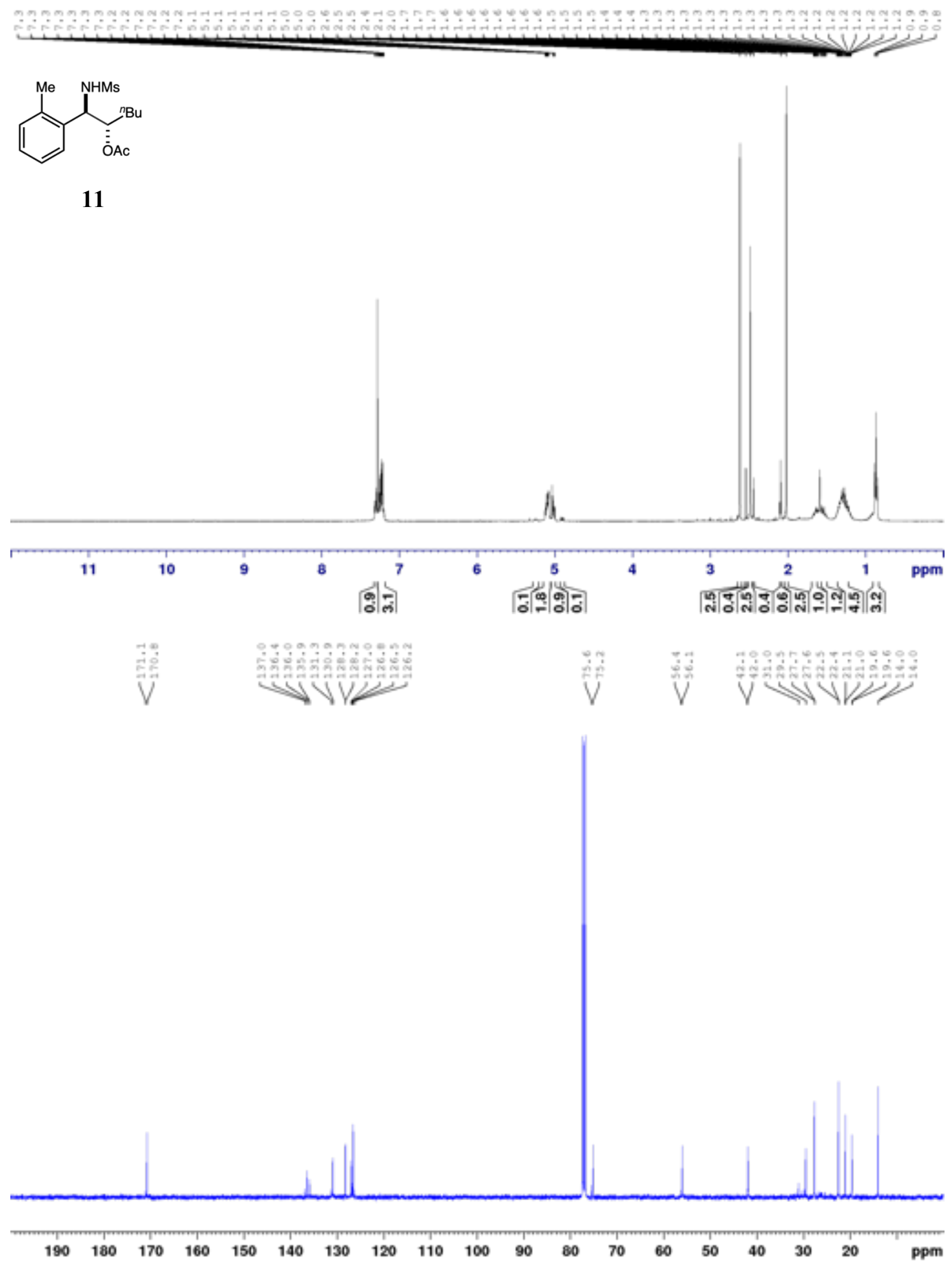




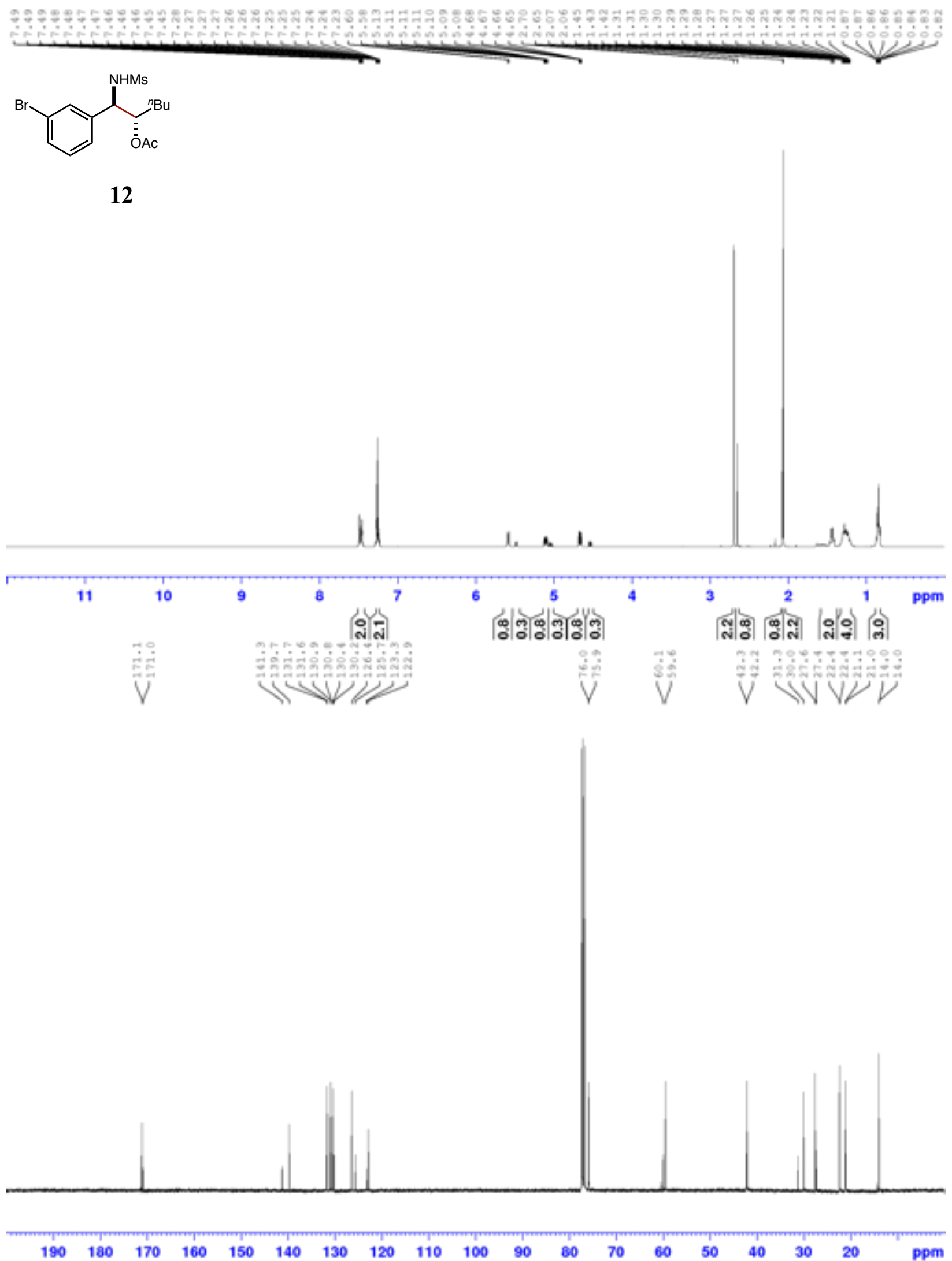




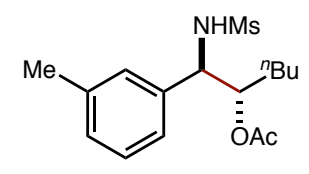

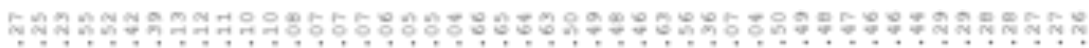

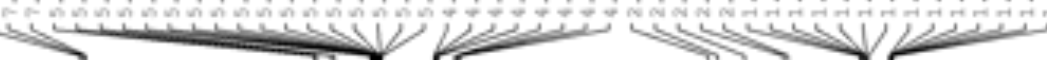

13
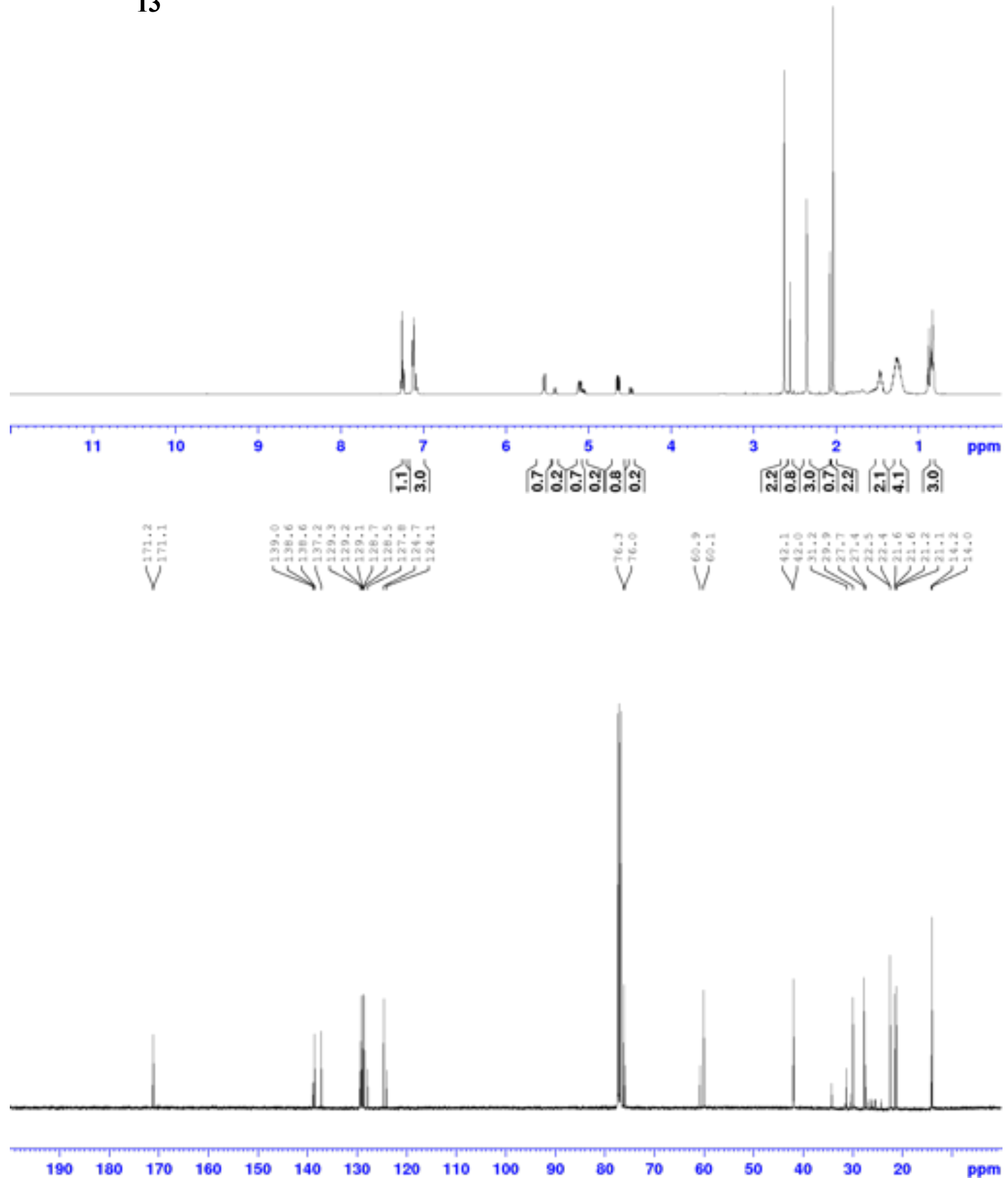


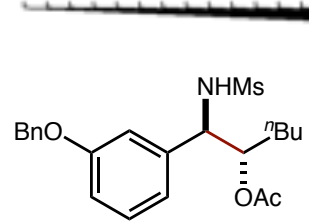

14
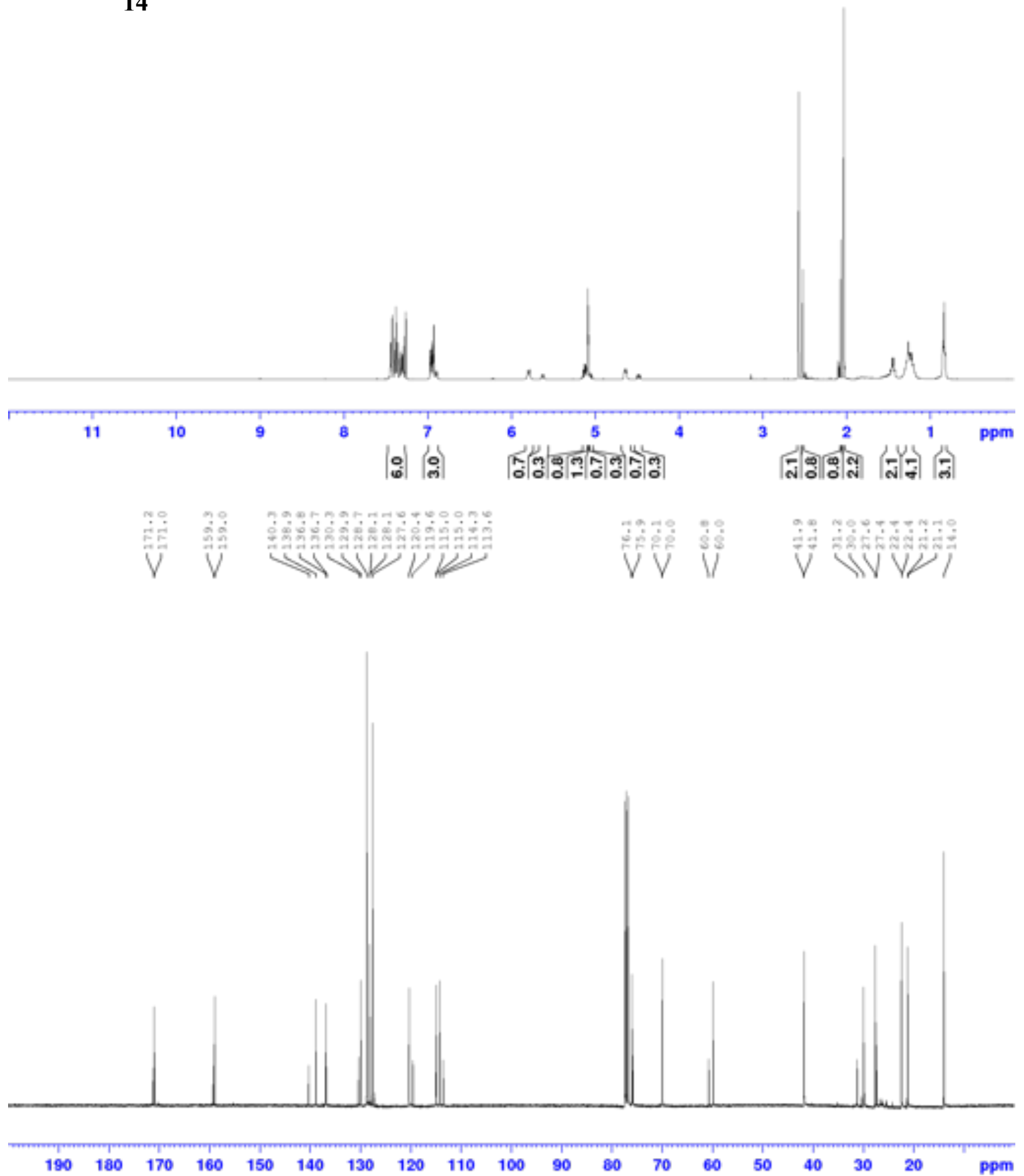


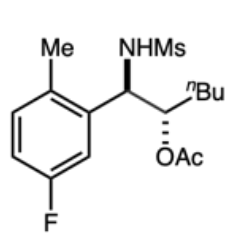

15
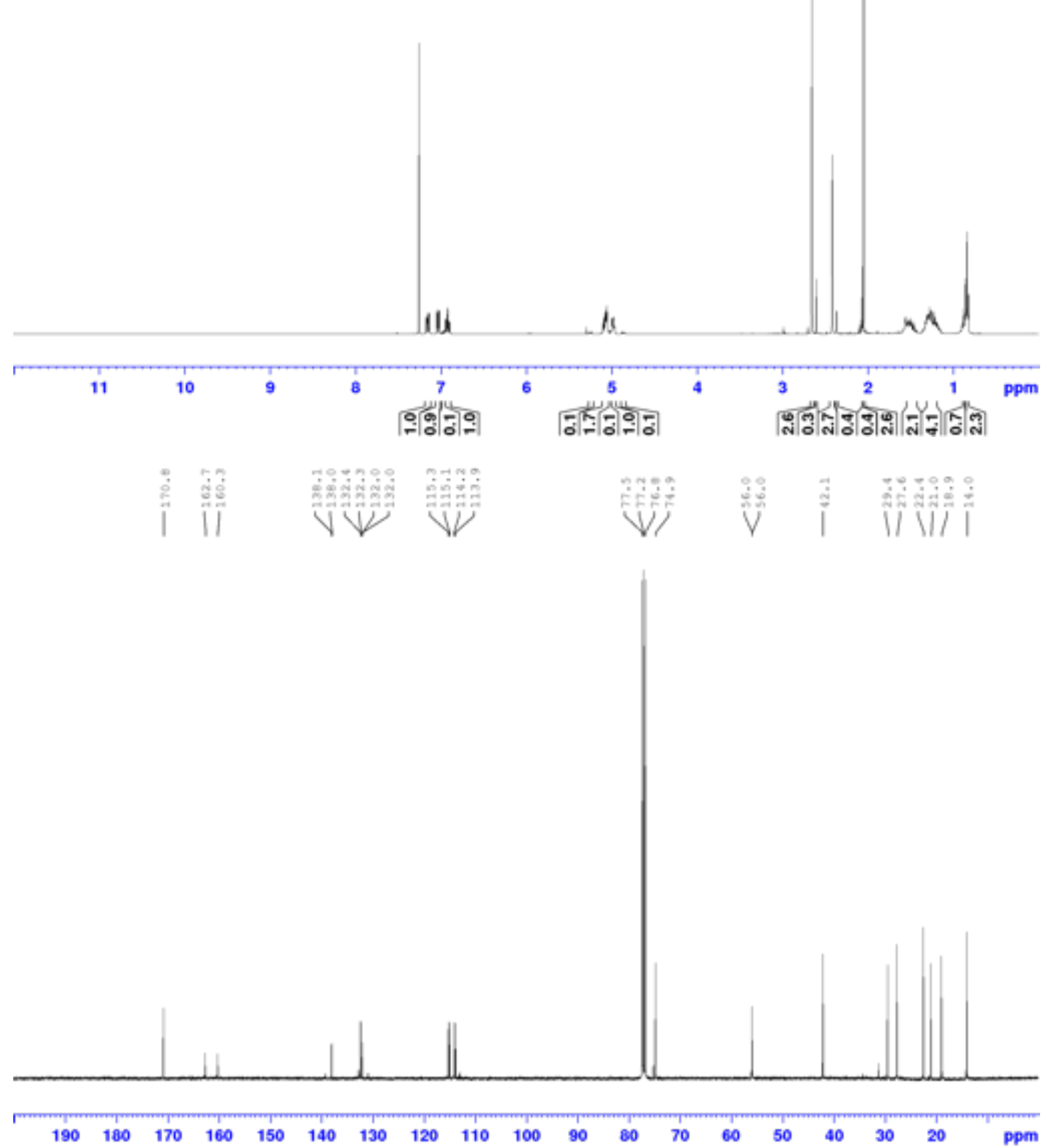


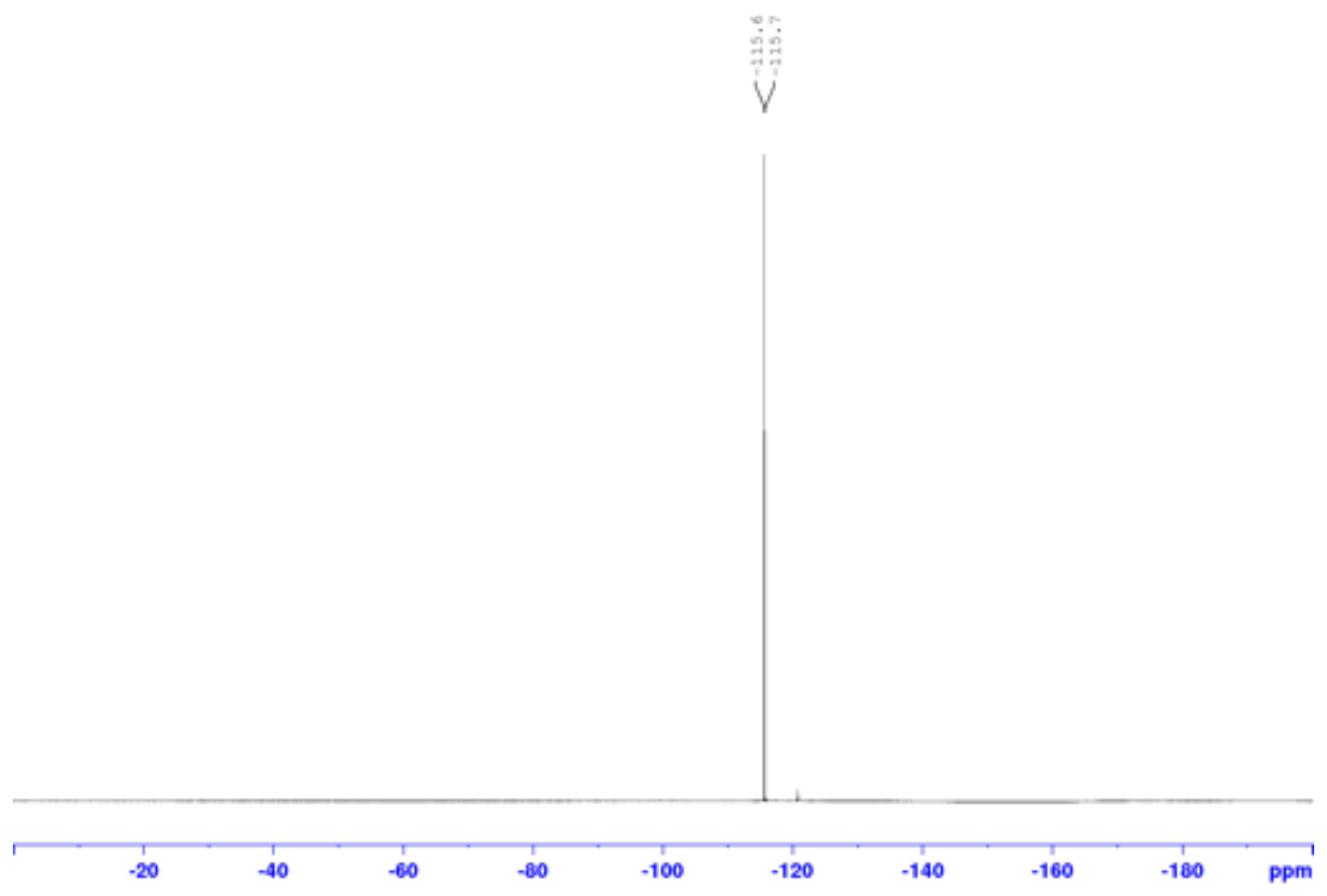




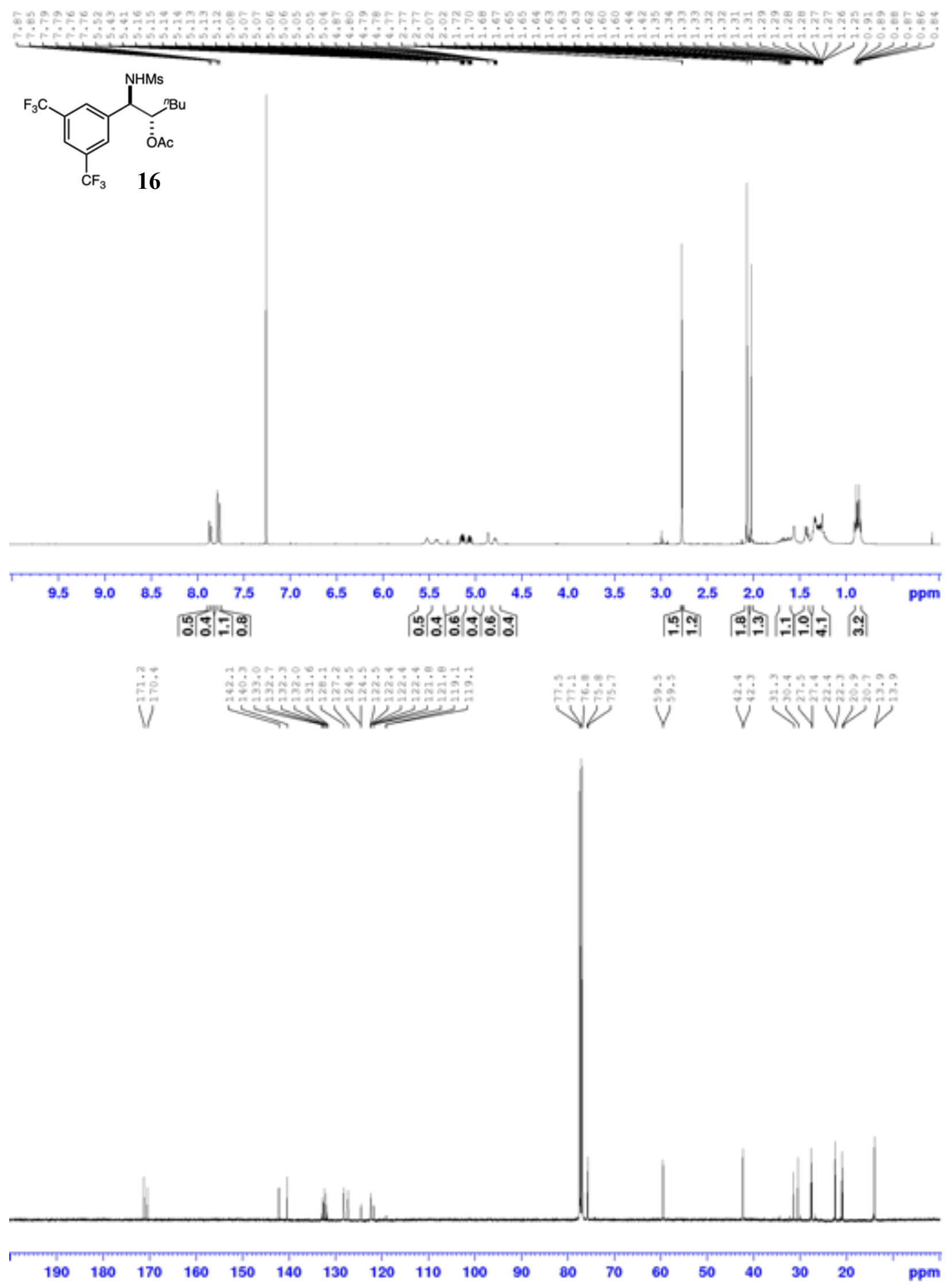




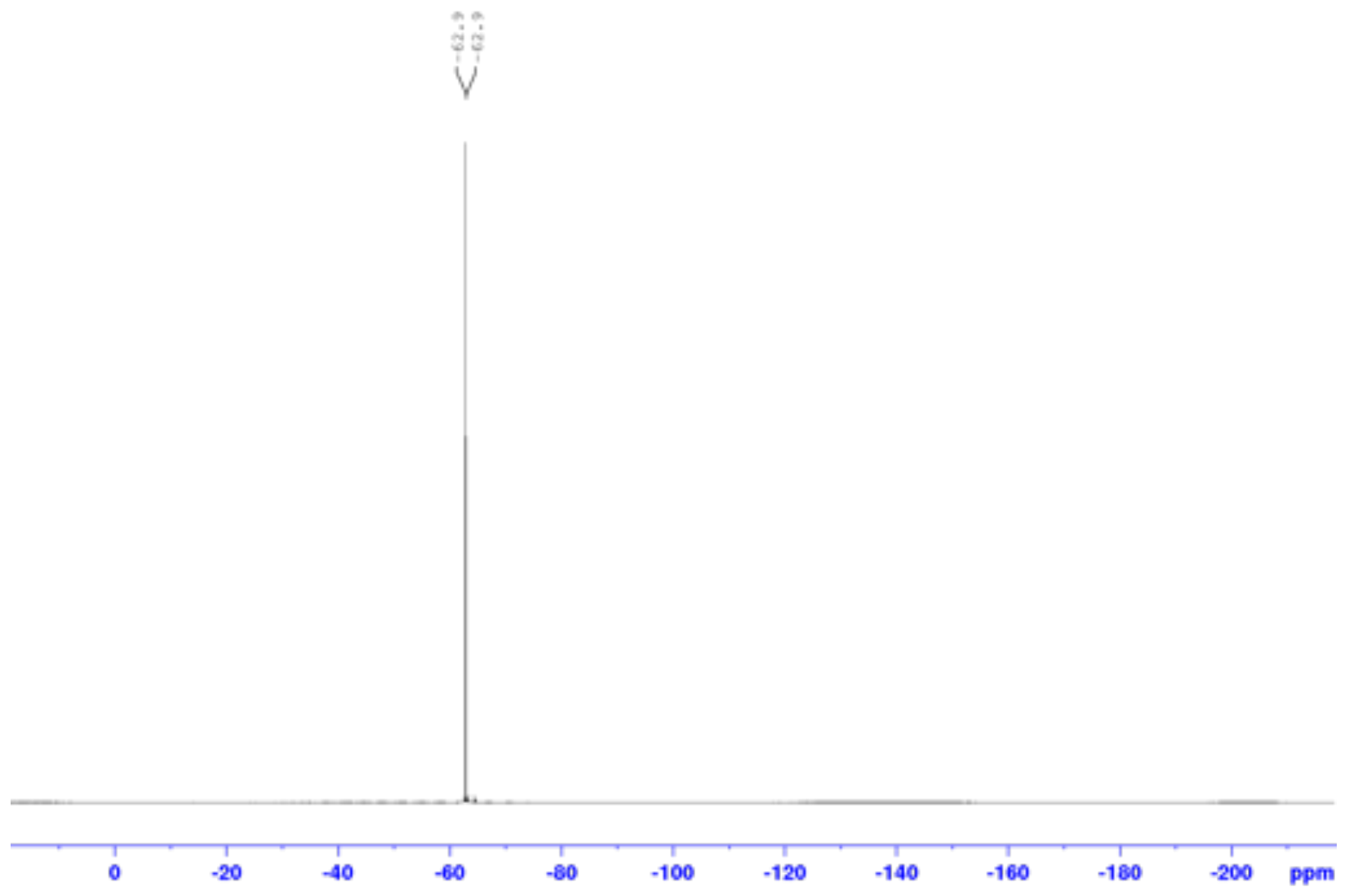


<smiles>CC(C)(C)[C@@H](N)C(N)c1ccc(F)nc1F</smiles>

17
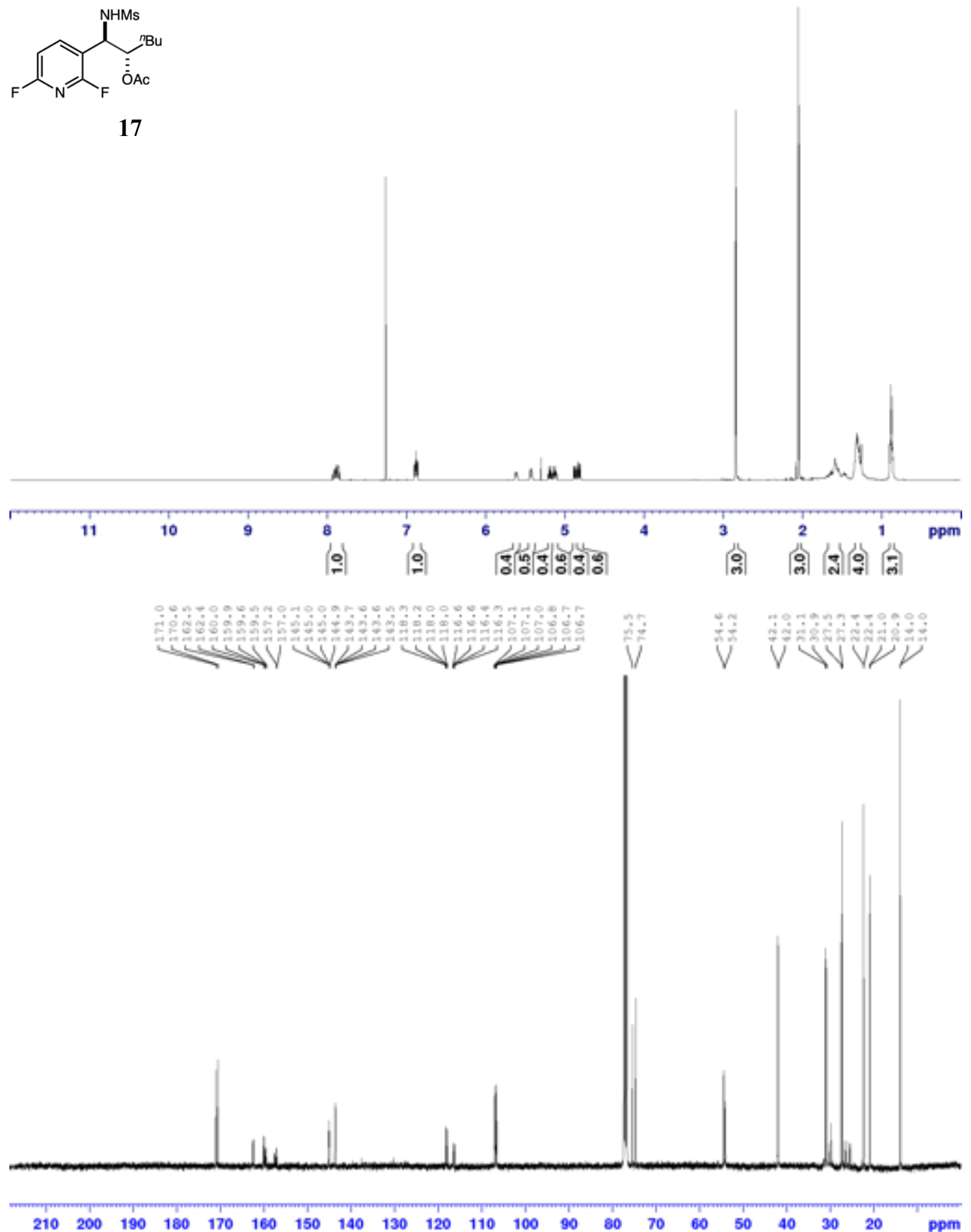


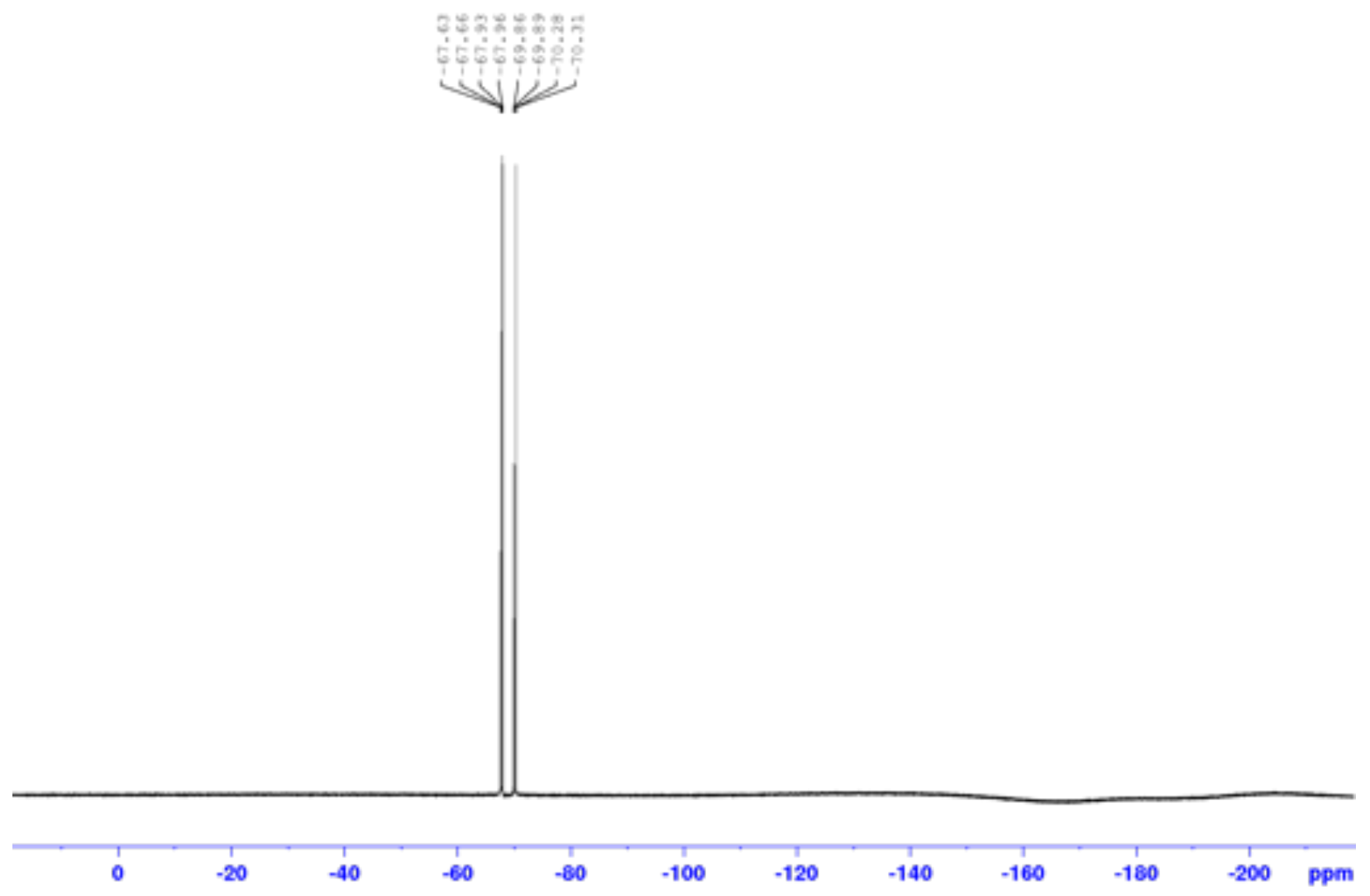



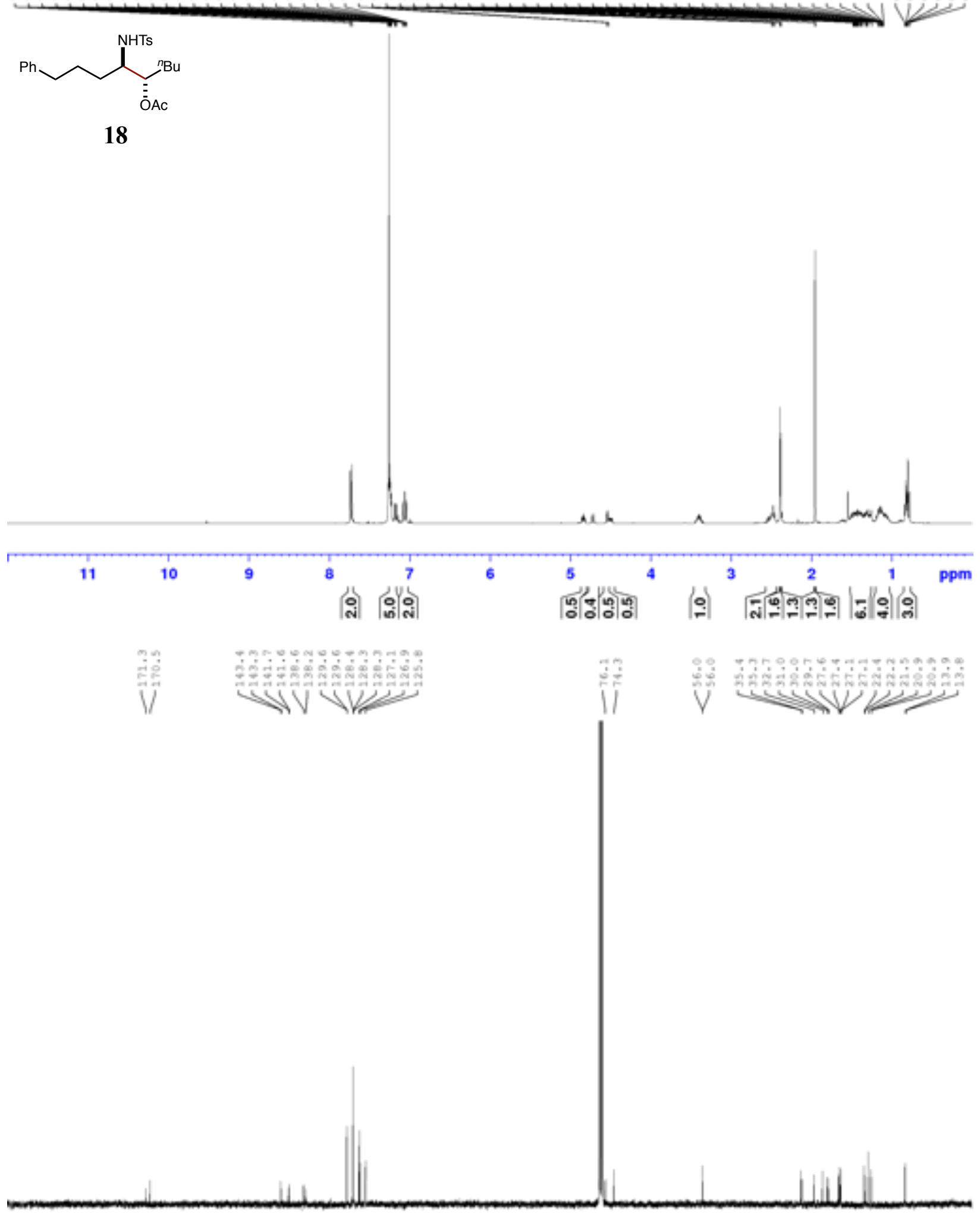

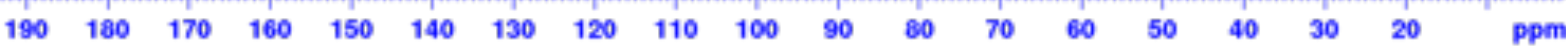




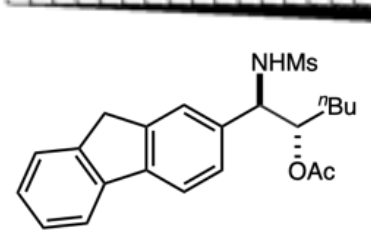

19

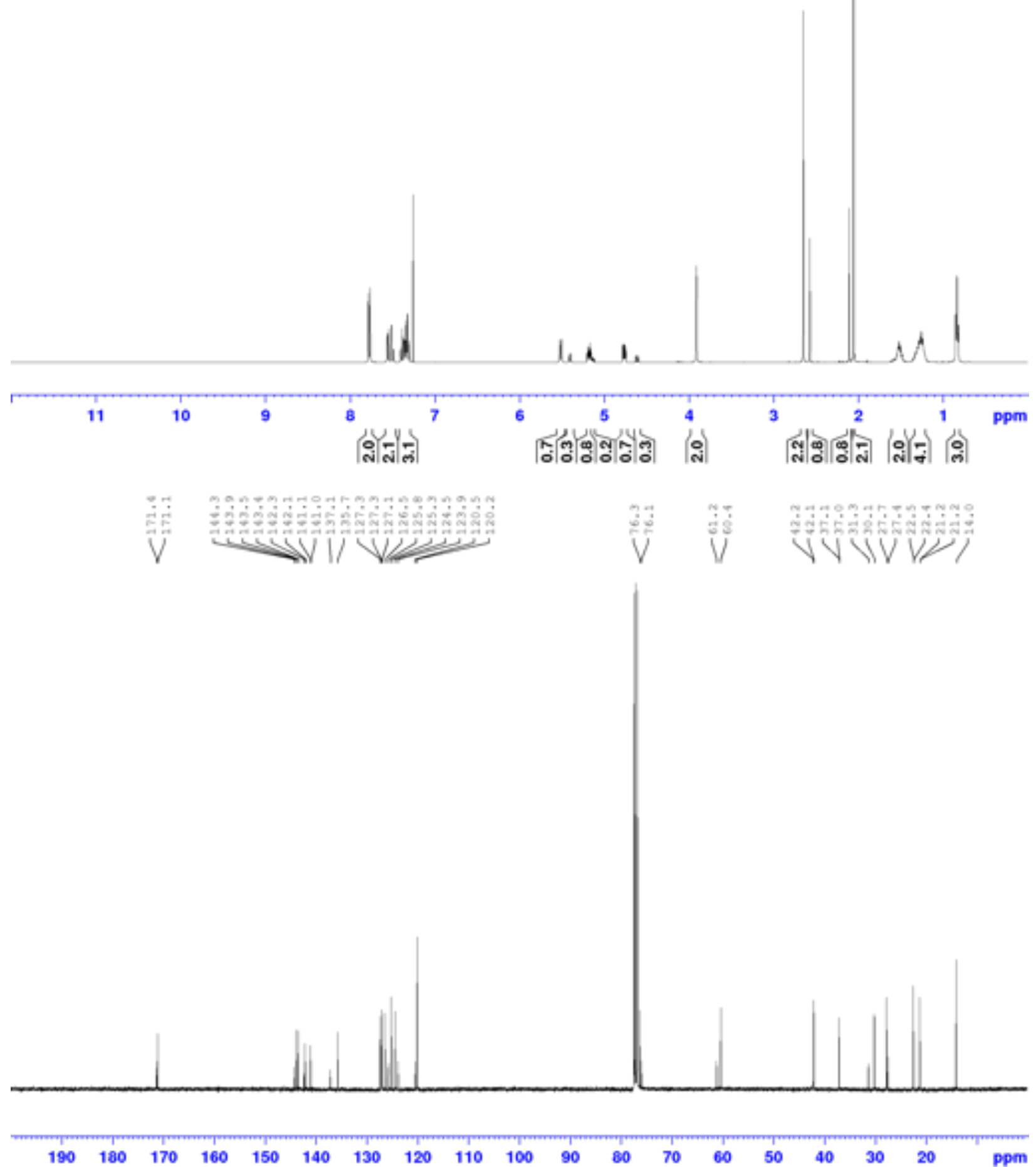


<smiles>NC(c1cc2ccccc2o1)C(Cc1ccccc1)C(=O)O</smiles>

20

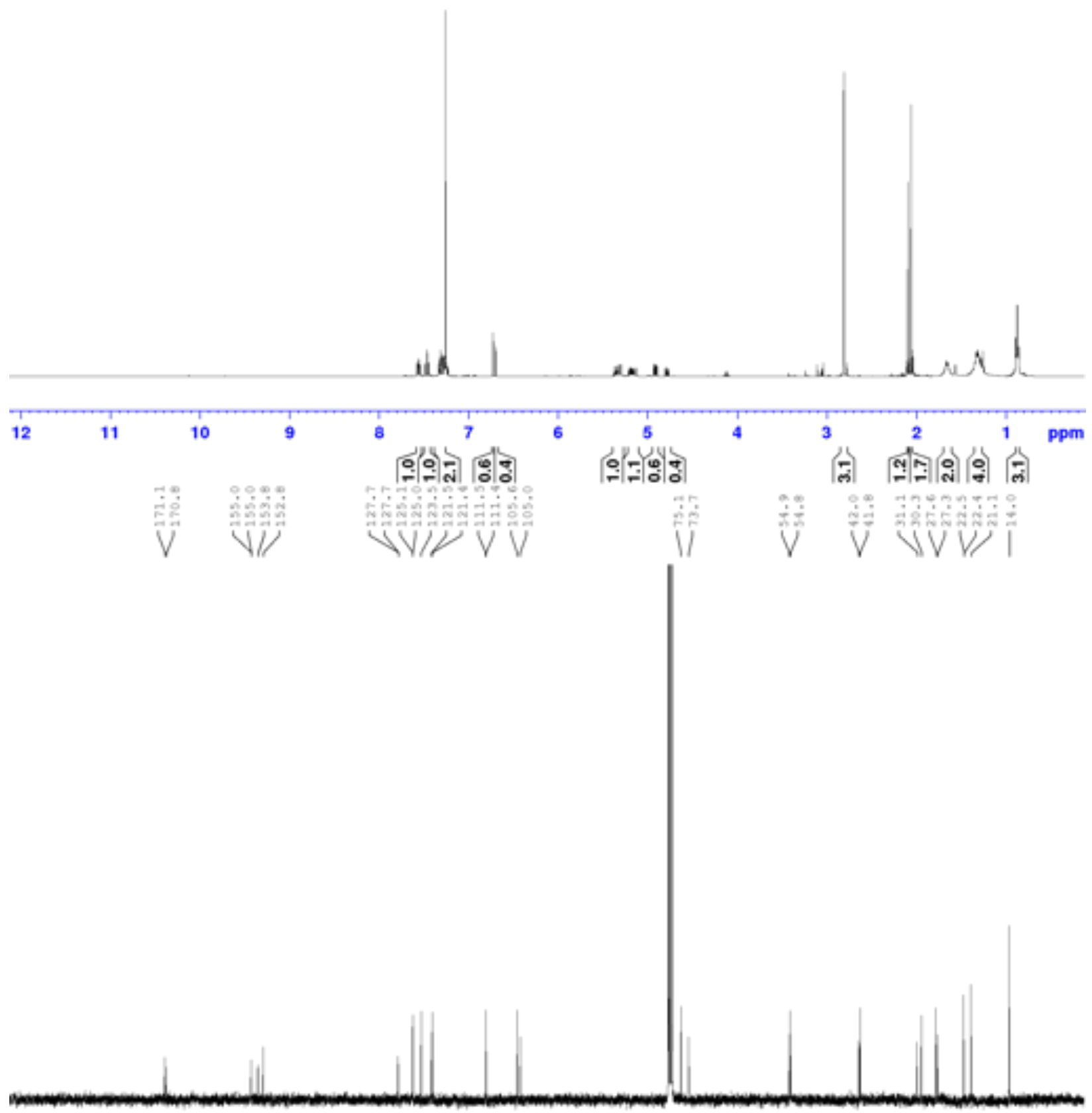

$\begin{array}{lllllllllllllllllll}190 & 180 & 170 & 160 & 150 & 140 & 130 & 120 & 110 & 100 & 90 & 80 & 70 & 60 & 50 & 40 & 30 & 20 & \mathrm{ppm}\end{array}$ 
<smiles>O=C(O[Na])C([18O])C([18O])c1ccc2c(c1)CCC21OCCO1</smiles>

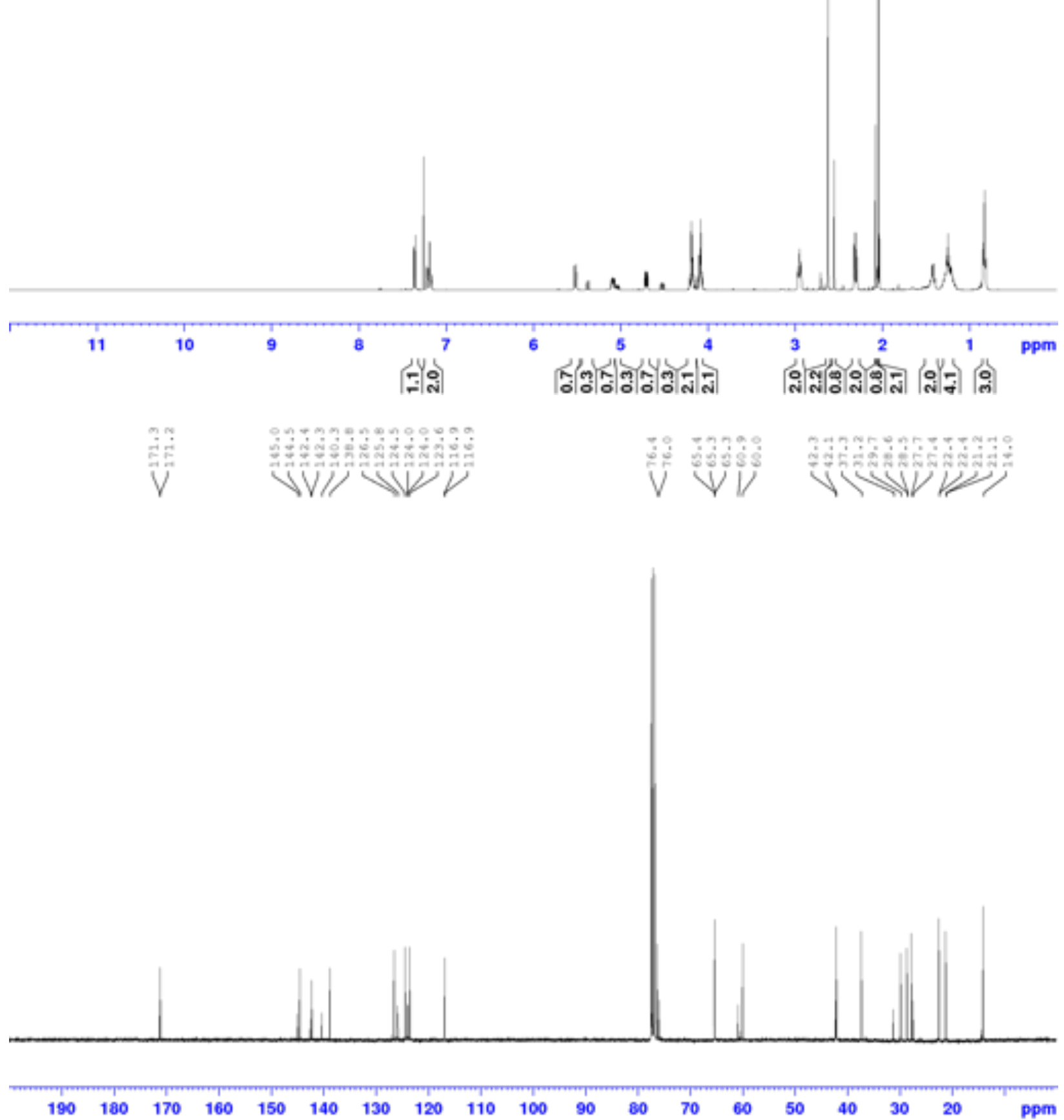



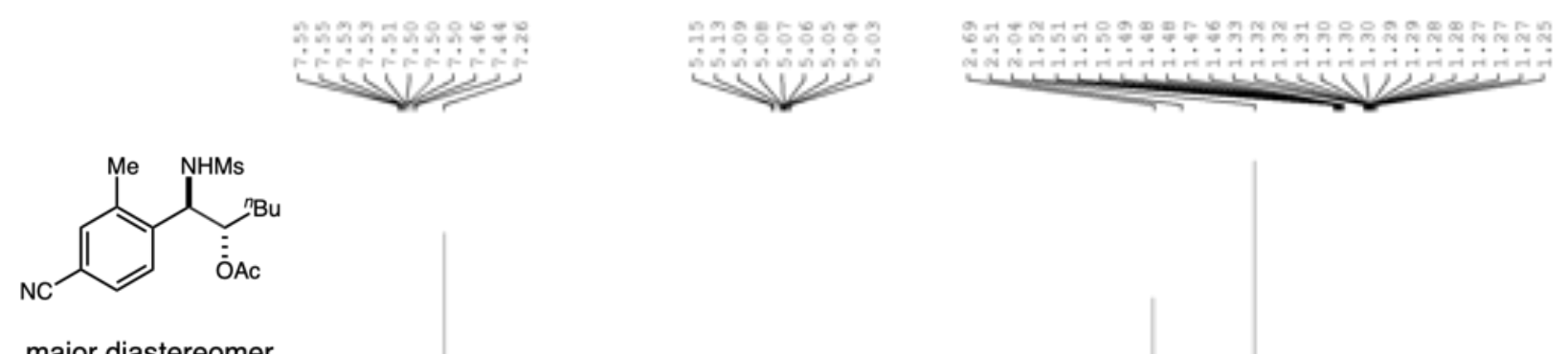

major diastereomer

22
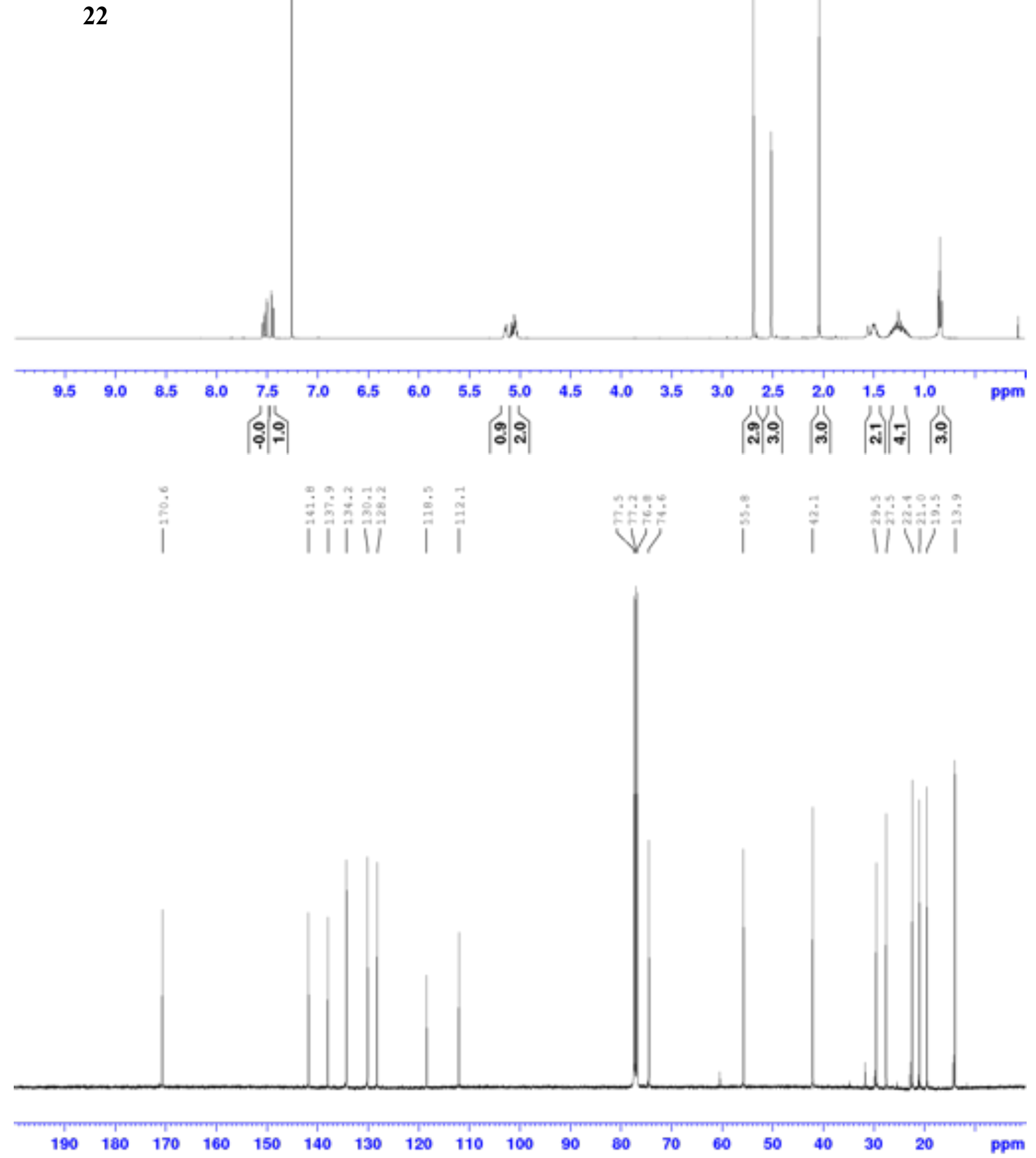

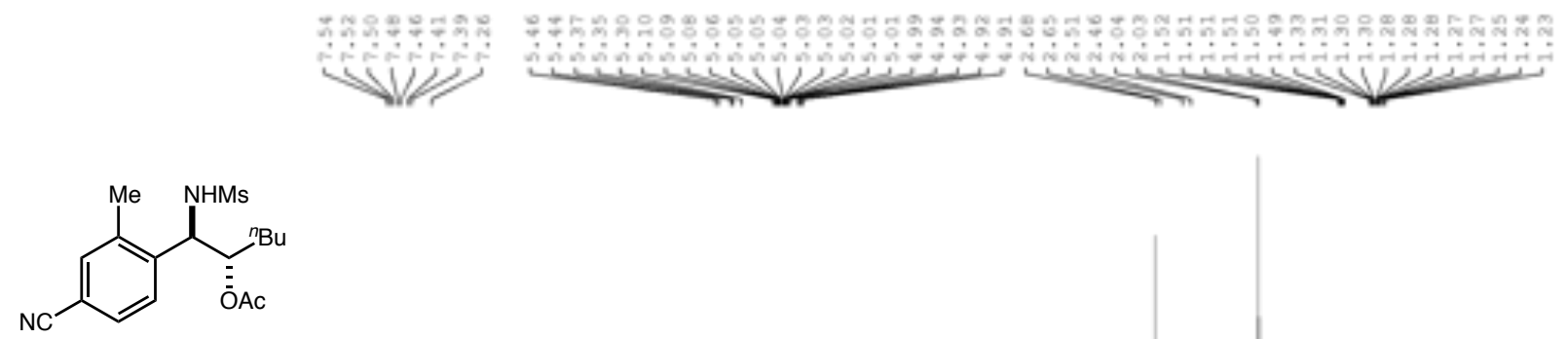

mixture of diastereomers

22

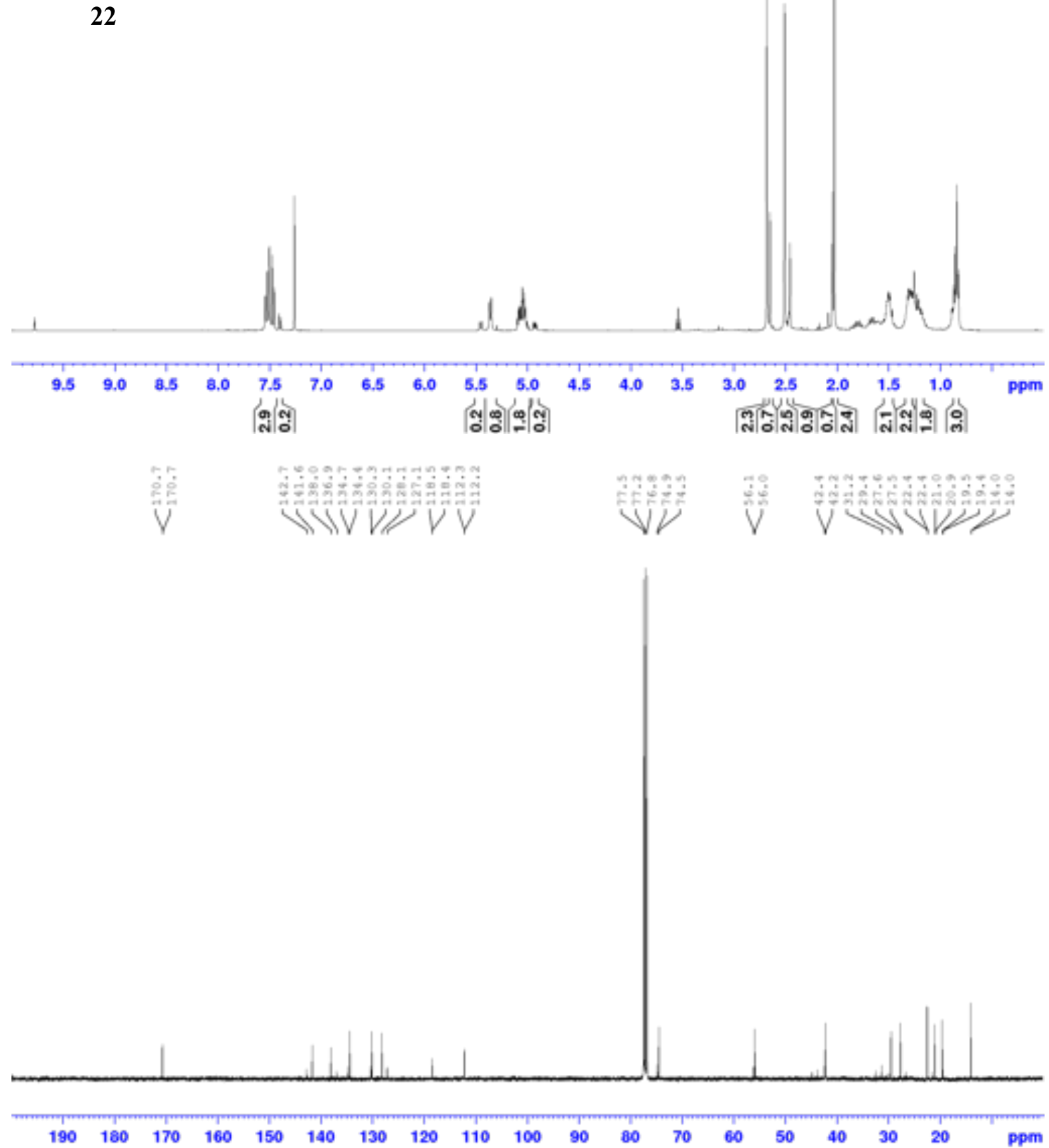



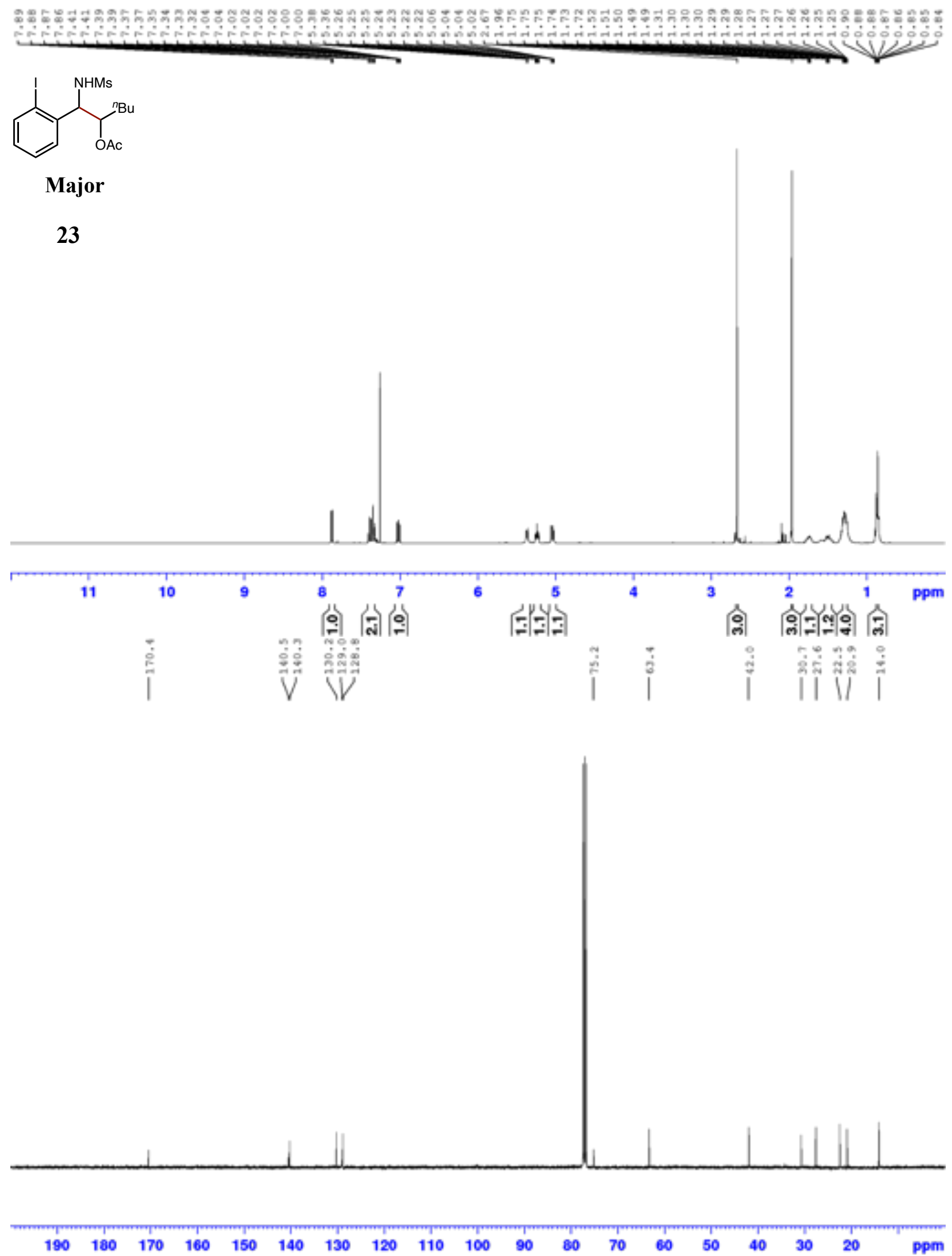
<smiles>CC(=O)OC(C(NS(C)(=O)=O)c1ccccc1I)C(C)(C)C</smiles>

Minor

23
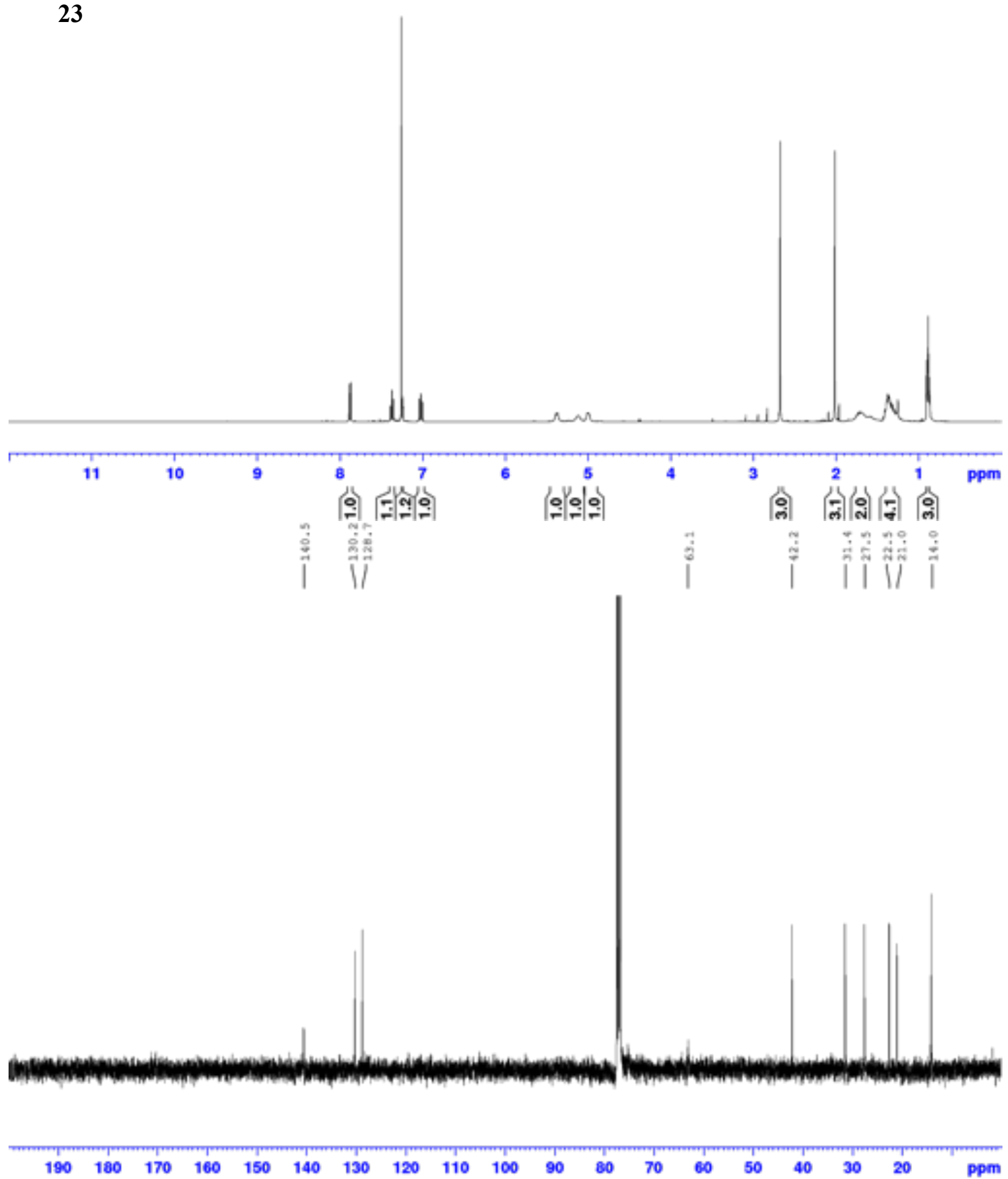

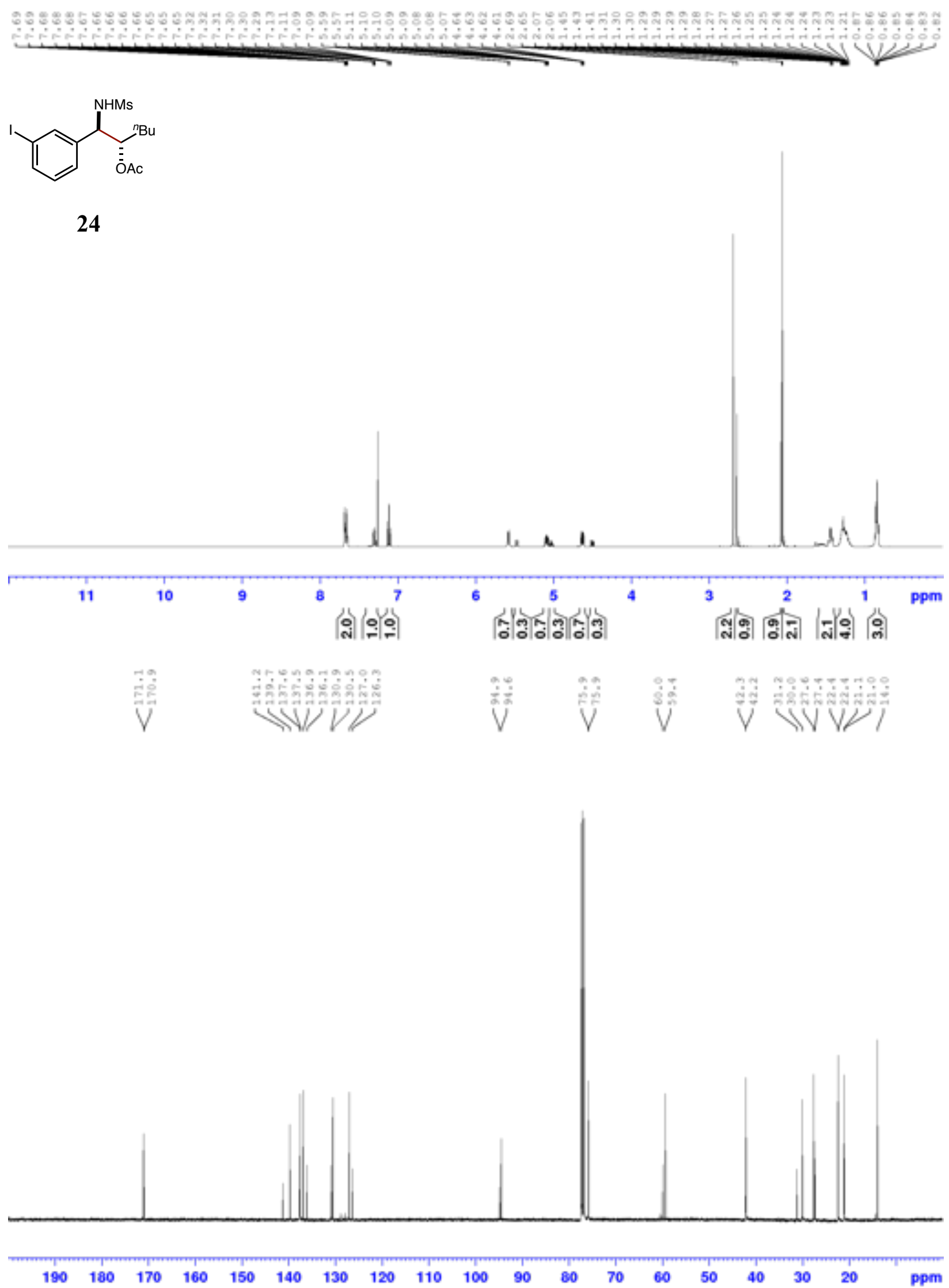
<smiles>CC(=O)OC(c1ccc(I)cc1)C(NS(C)(=O)=O)C(C)(C)C</smiles>

25

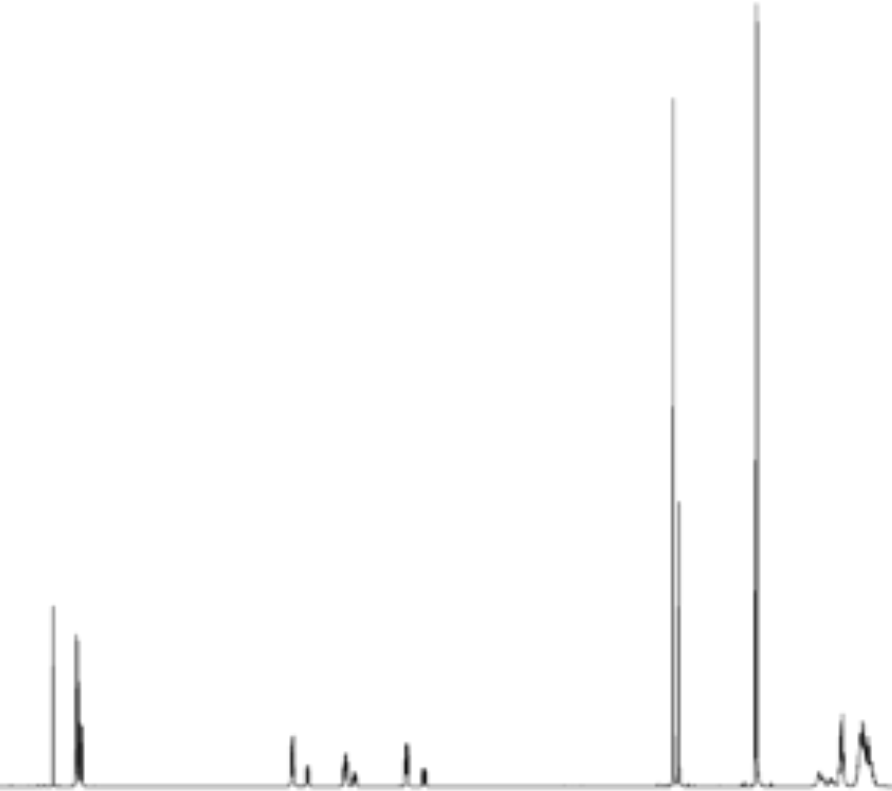

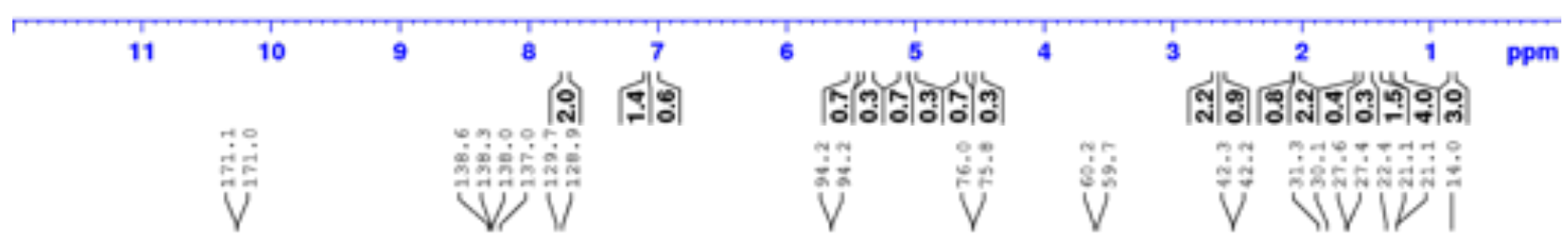

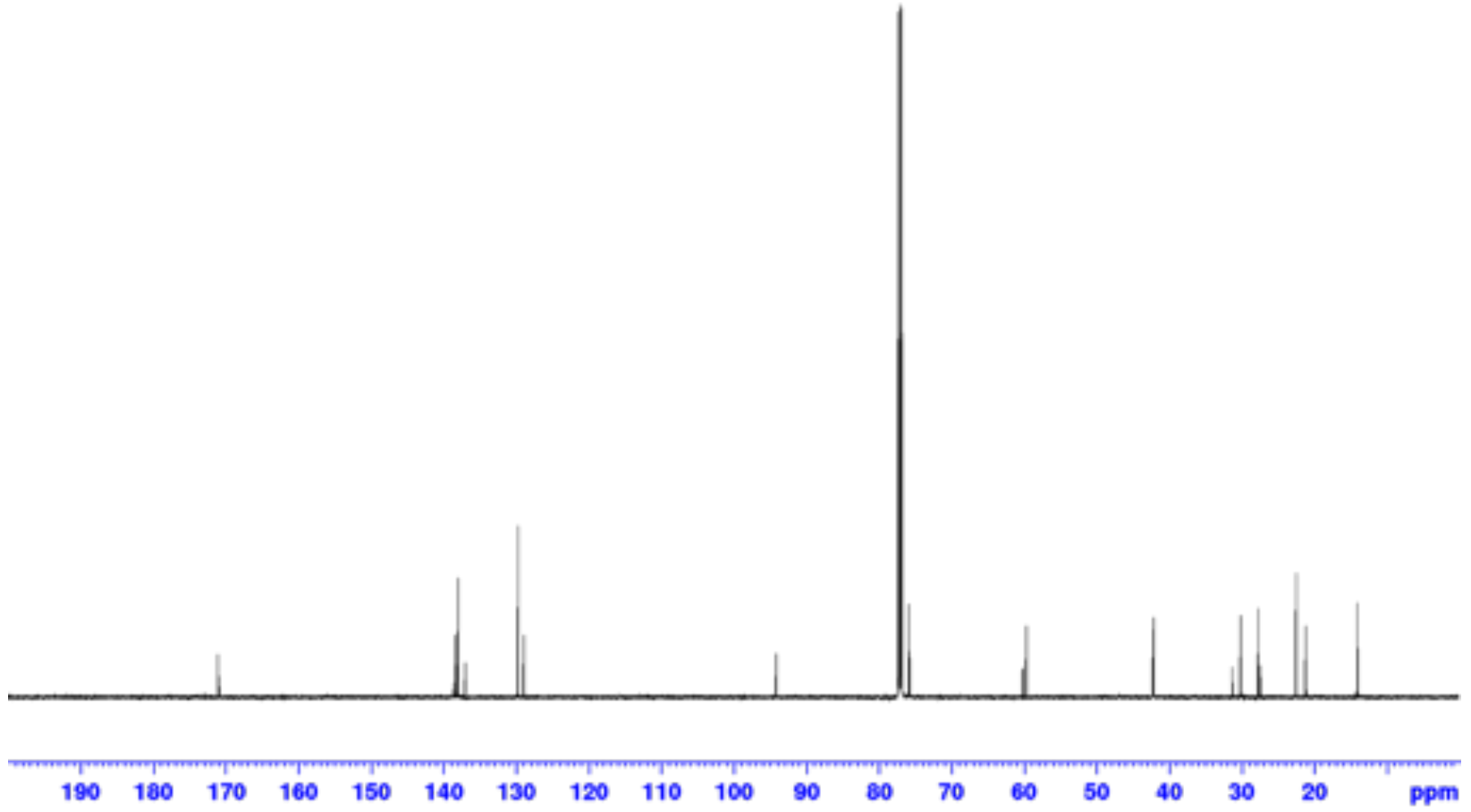


<smiles>CC(C)(C)O[C@H](C(N)C(N)c1cccc(C(=O)c2ccccc2)c1)C(C)(C)C</smiles>

26
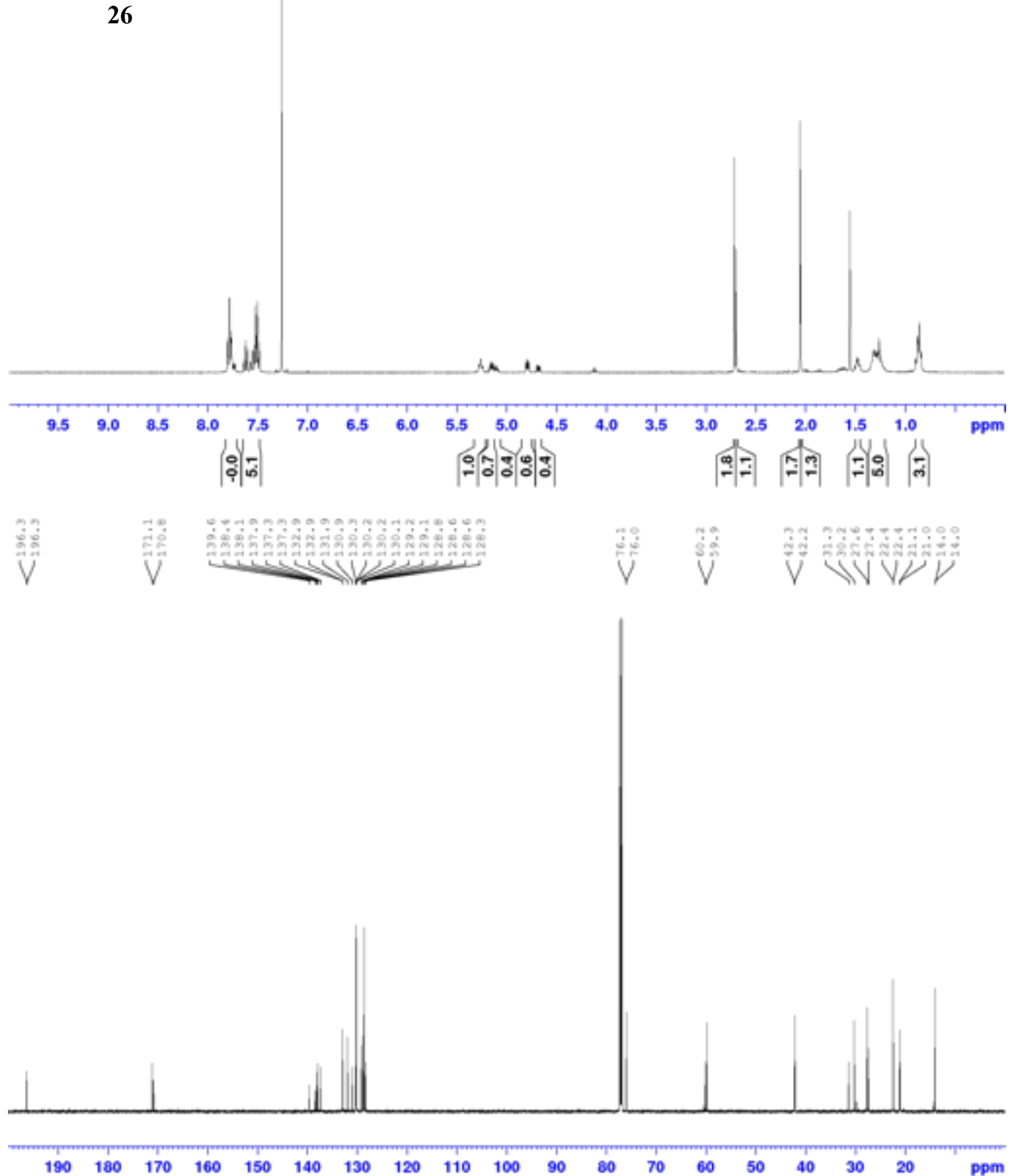


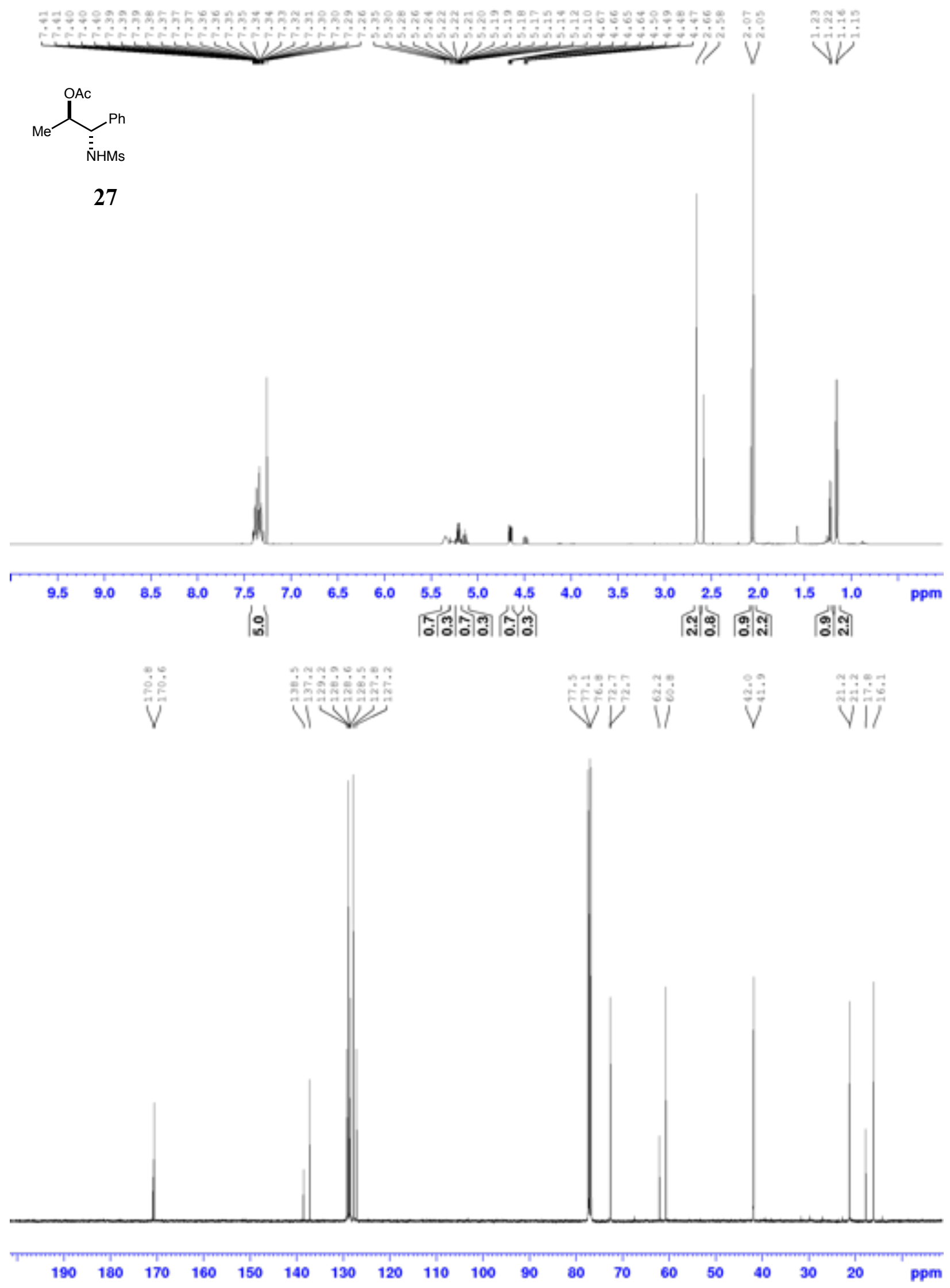




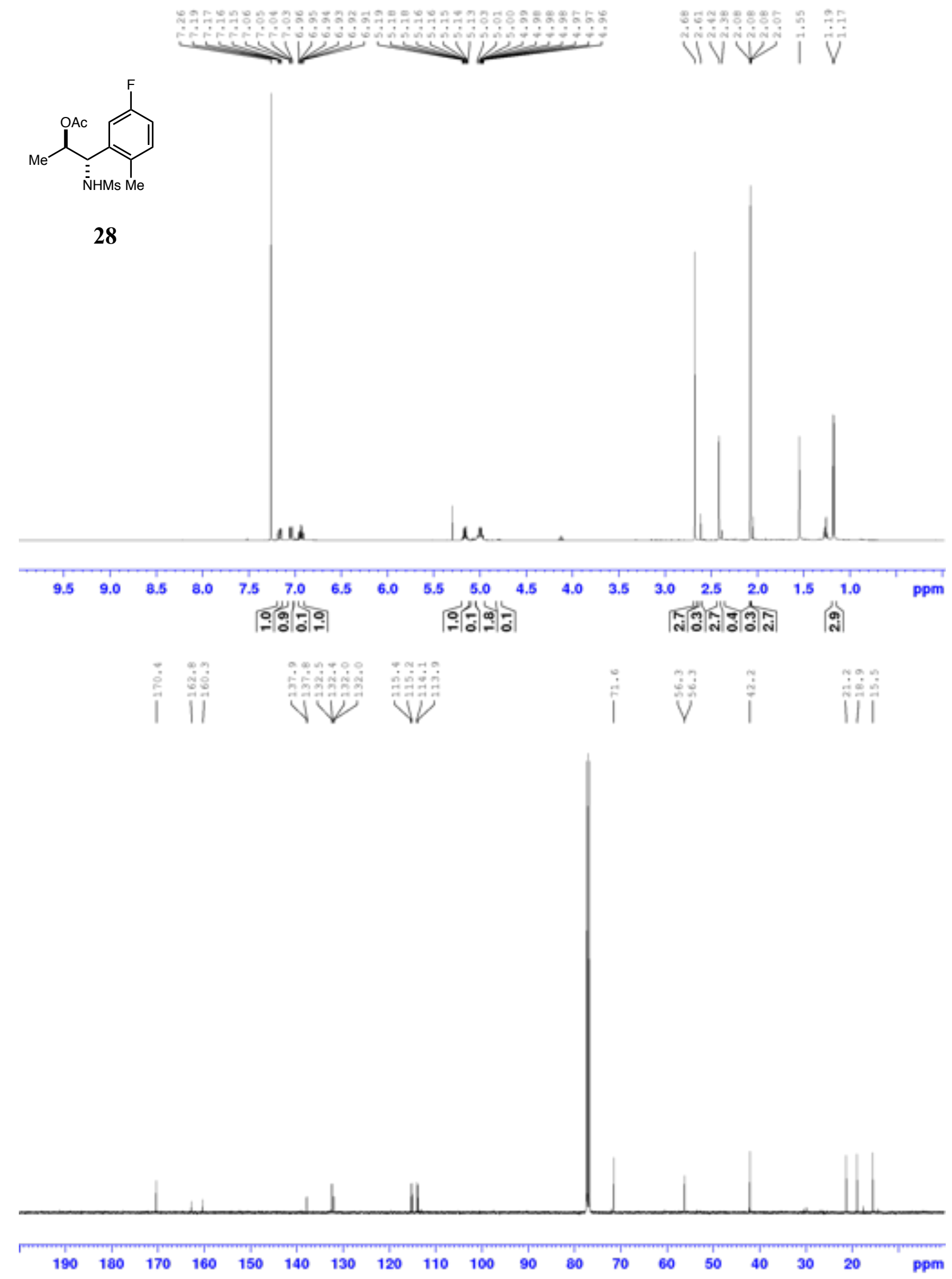



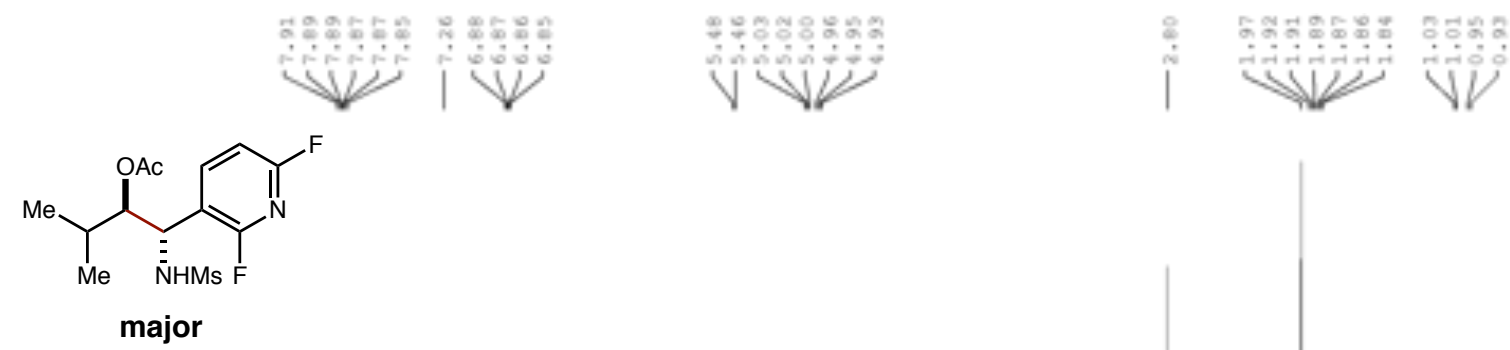

29
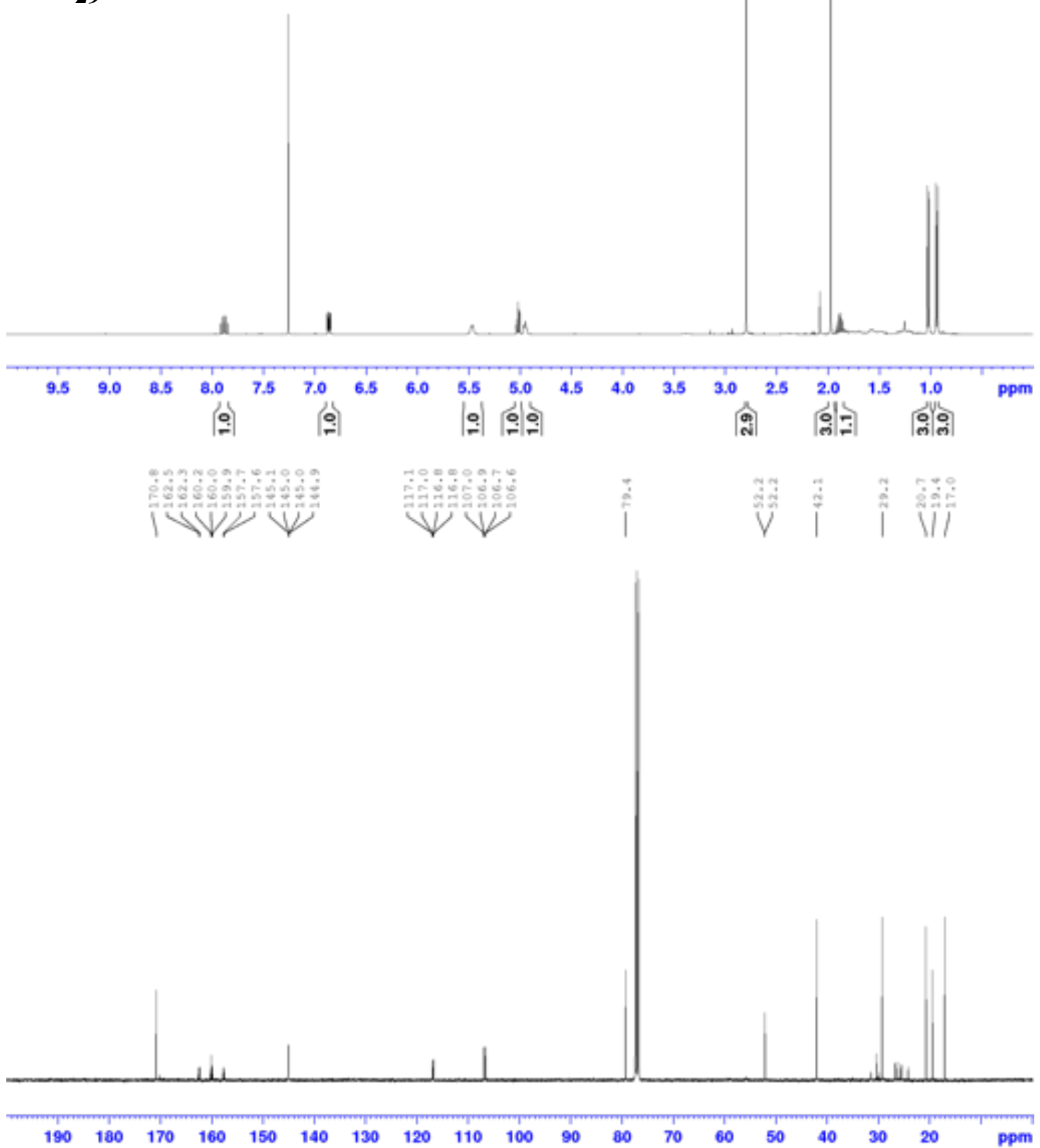


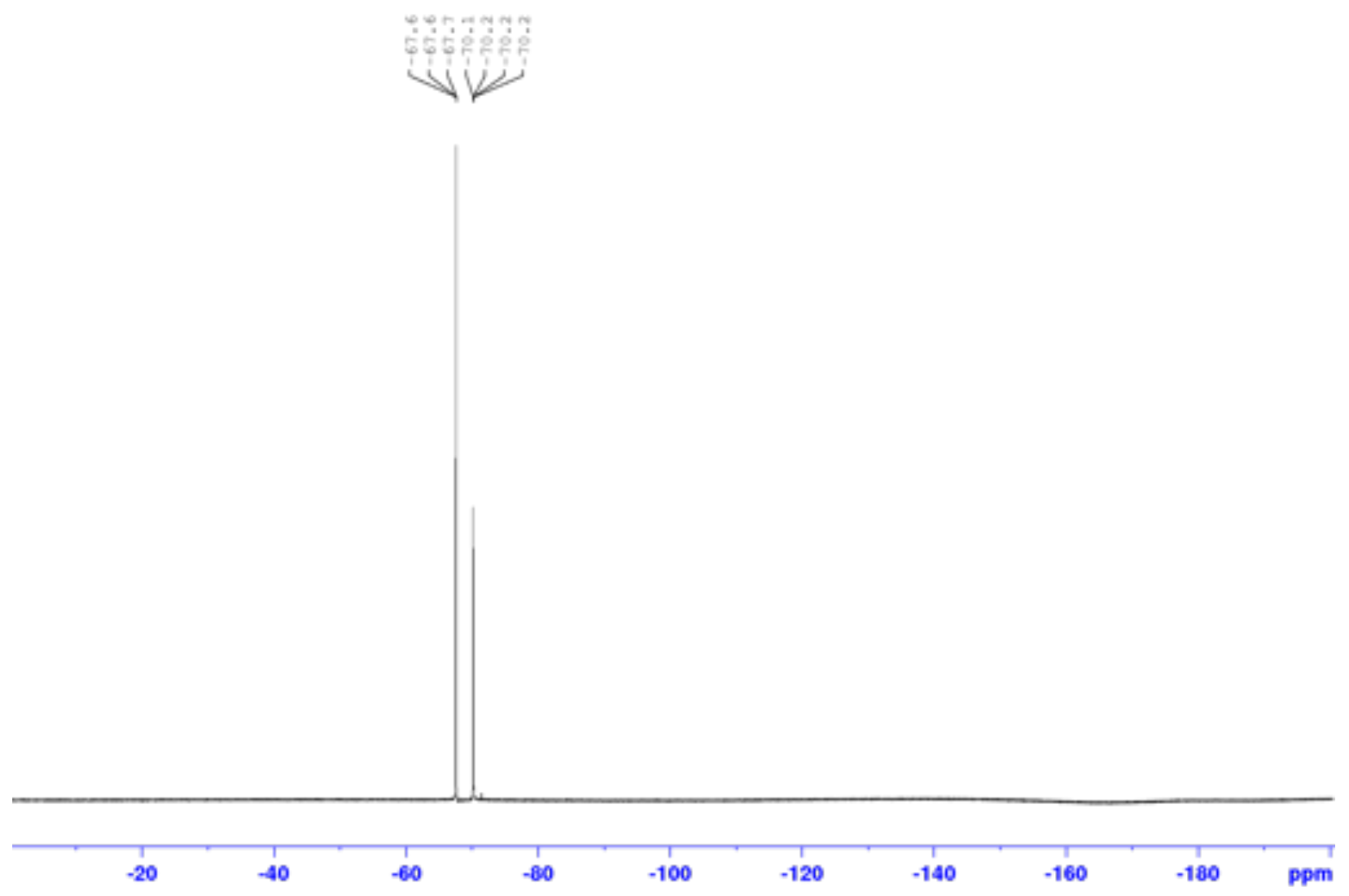




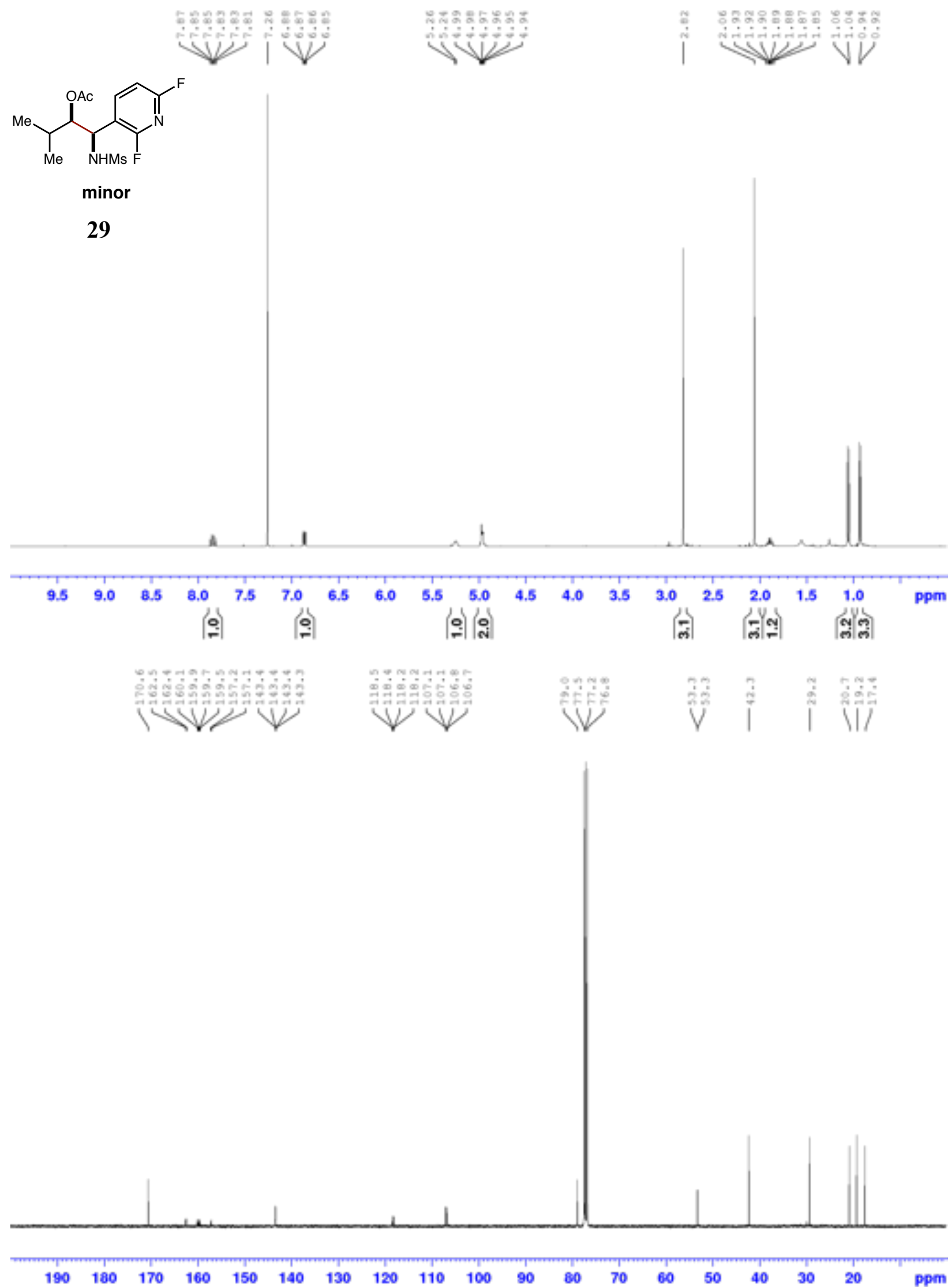




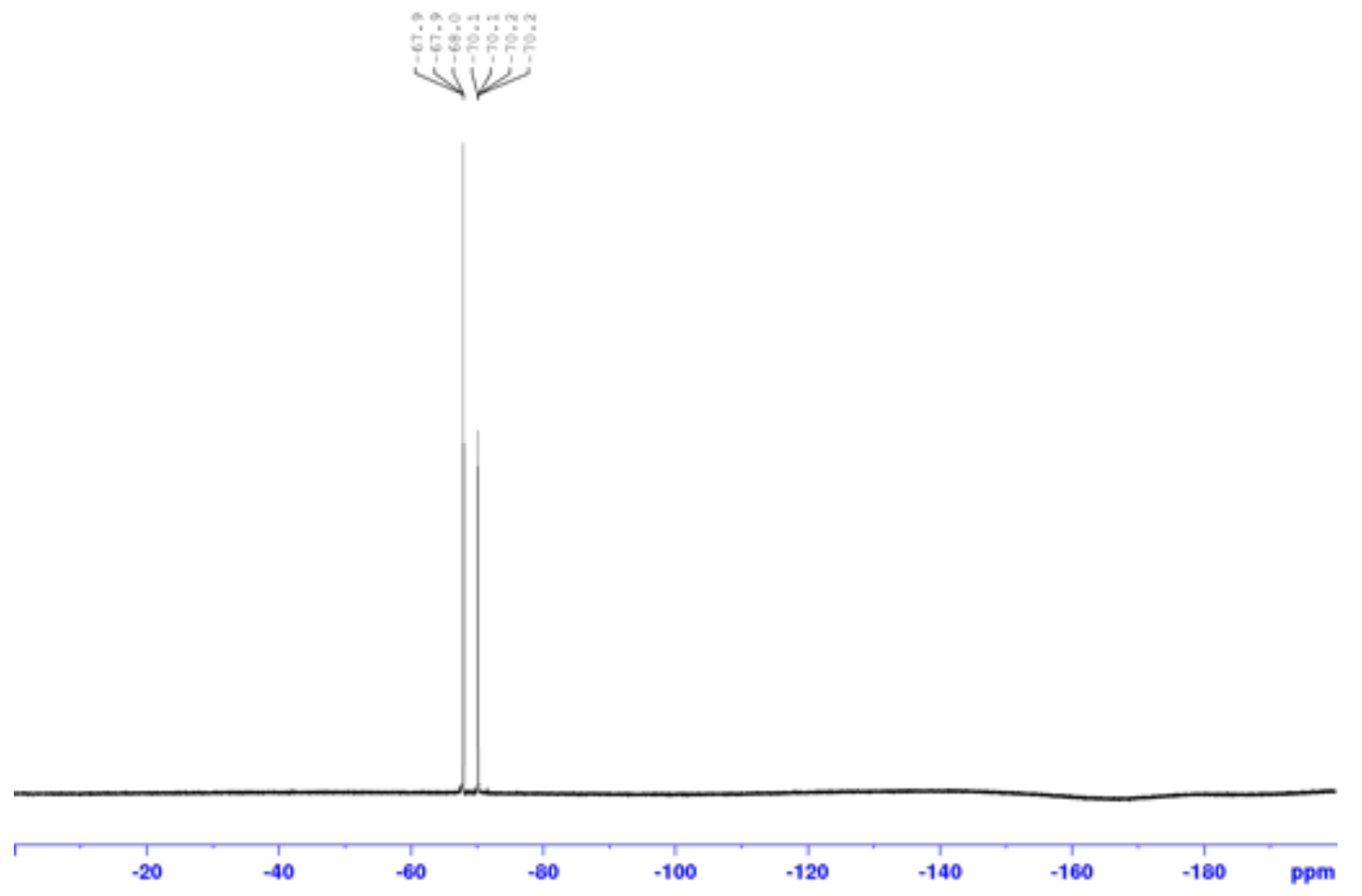

S112 


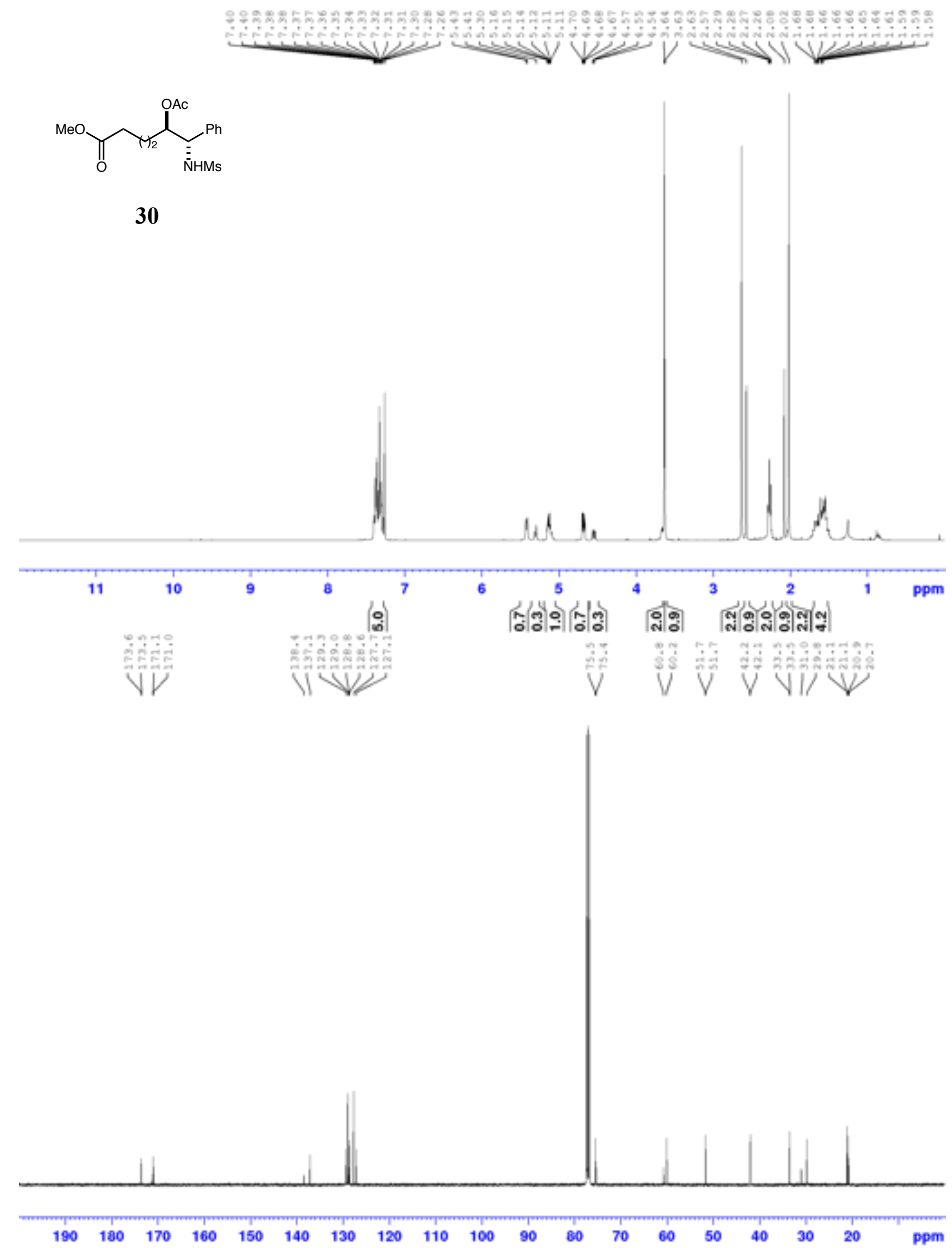


<smiles>CC(=O)OC(CCCCCC#N)C(N)c1ccccc1</smiles>

\section{1}
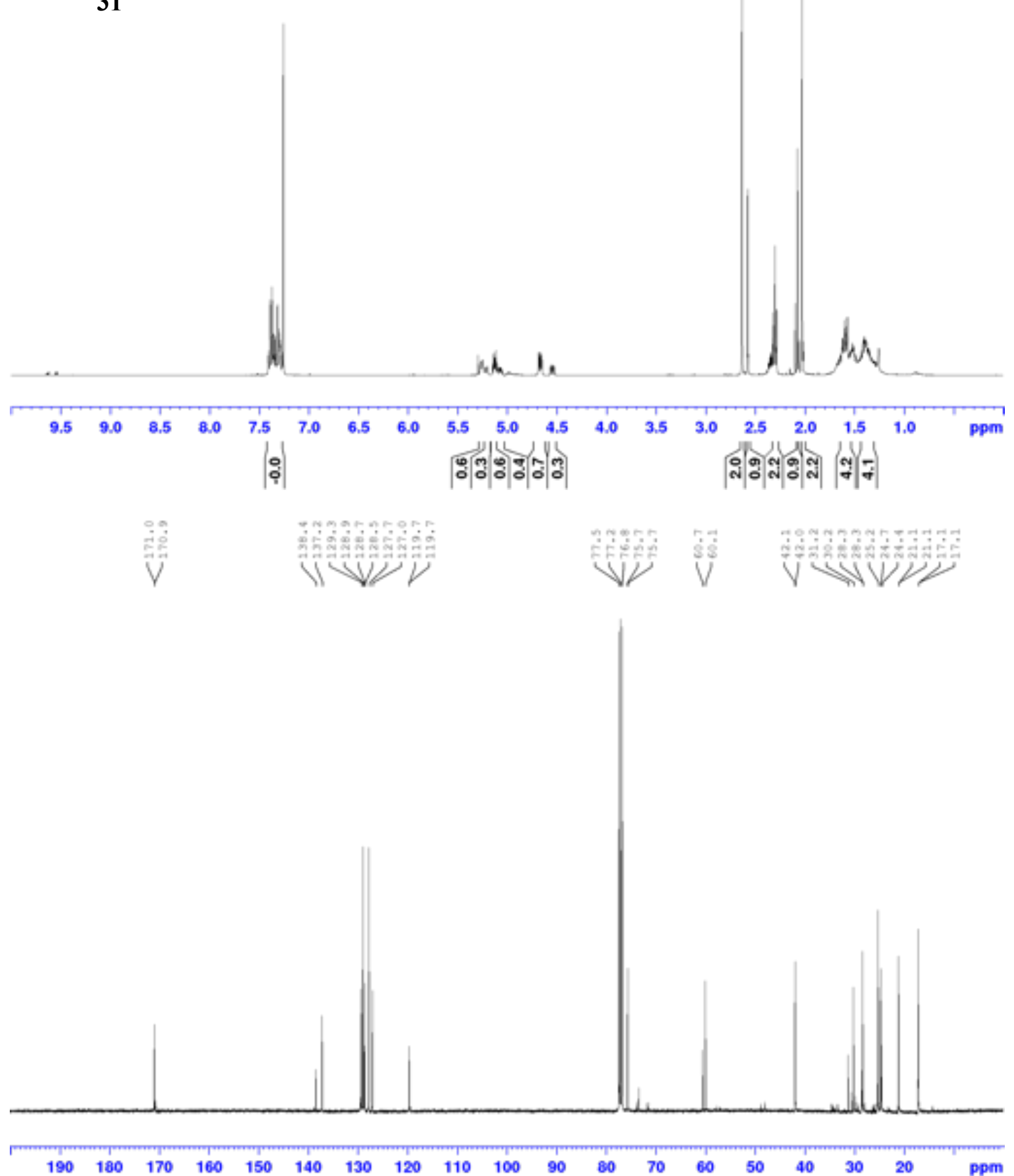


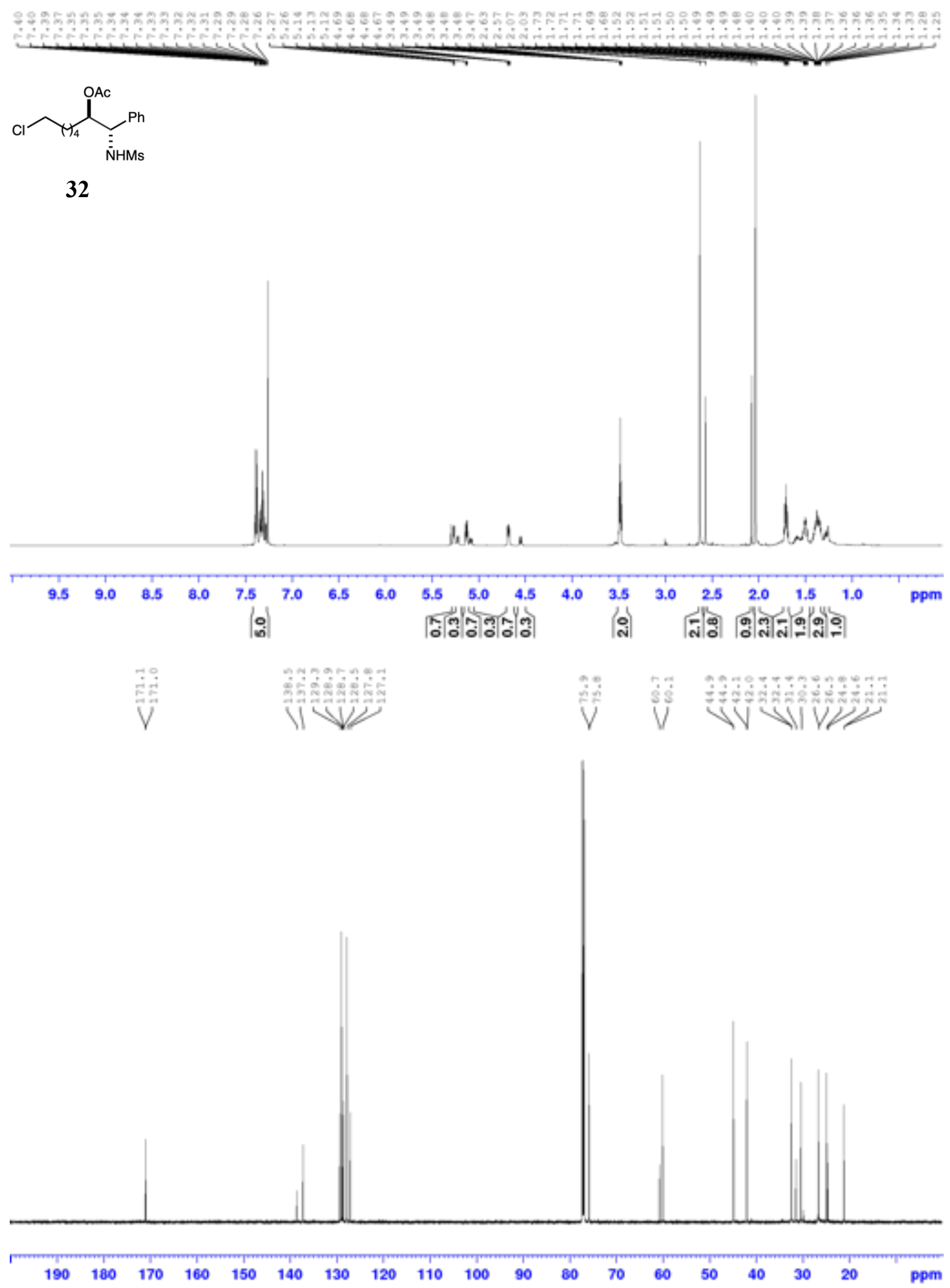




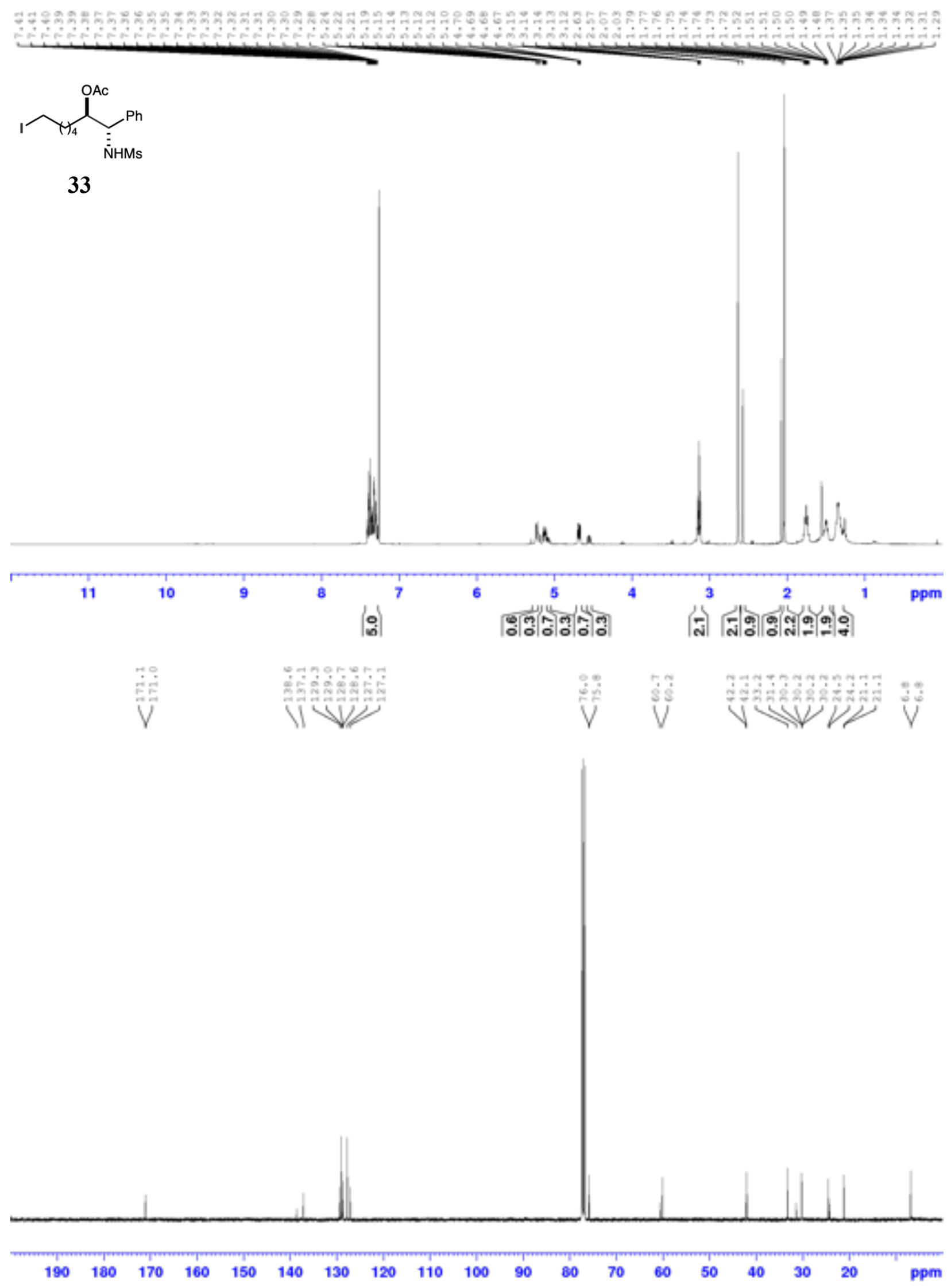




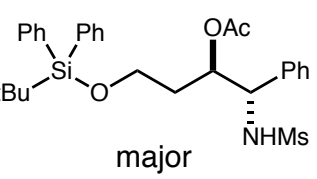

\section{4}
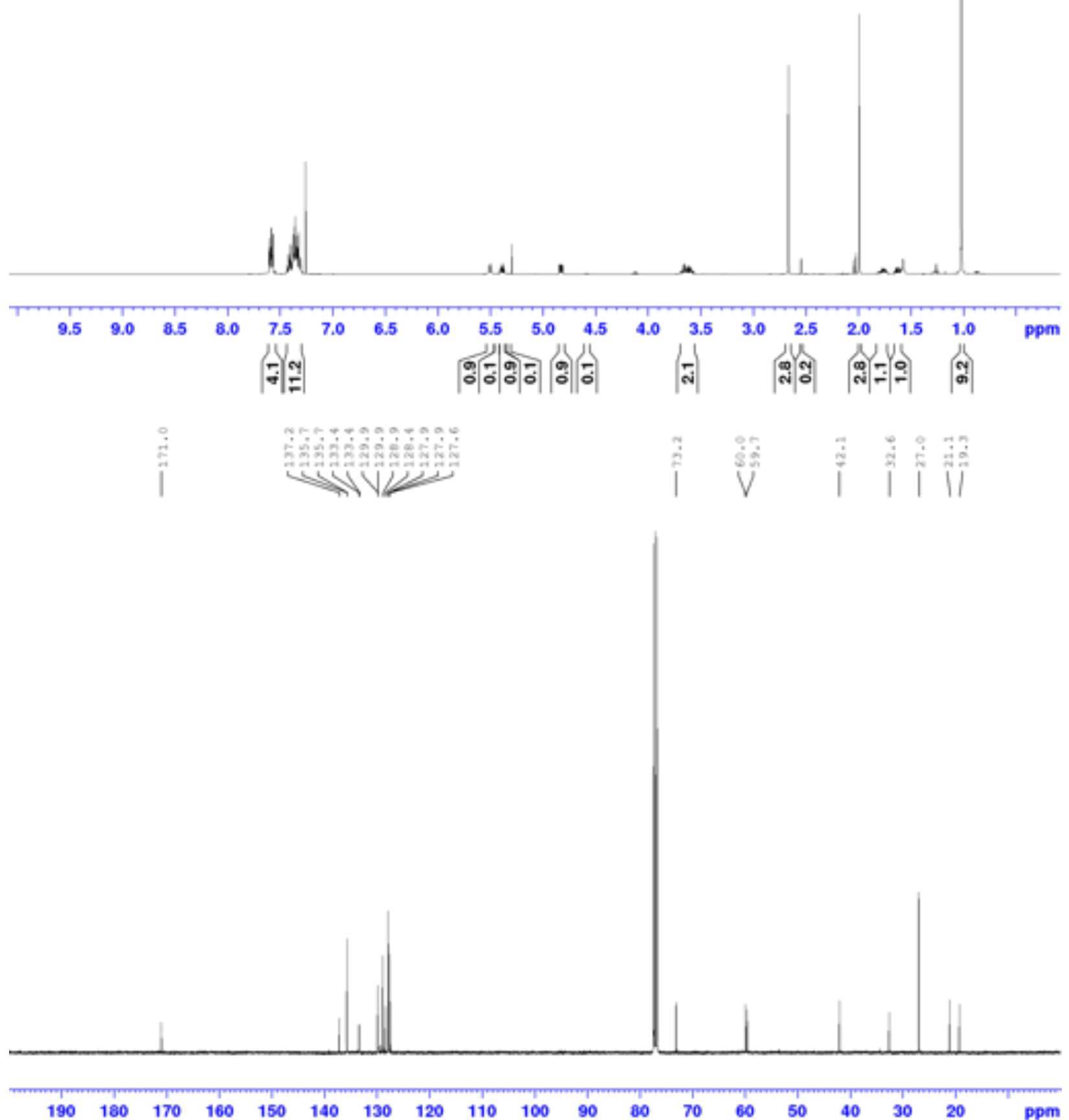
<smiles>CC(=O)OC(CCO[Si](CC(C)C)(c1ccccc1)c1ccccc1)[C@@H](N)c1ccccc1</smiles>

mixture of diastereomers

34
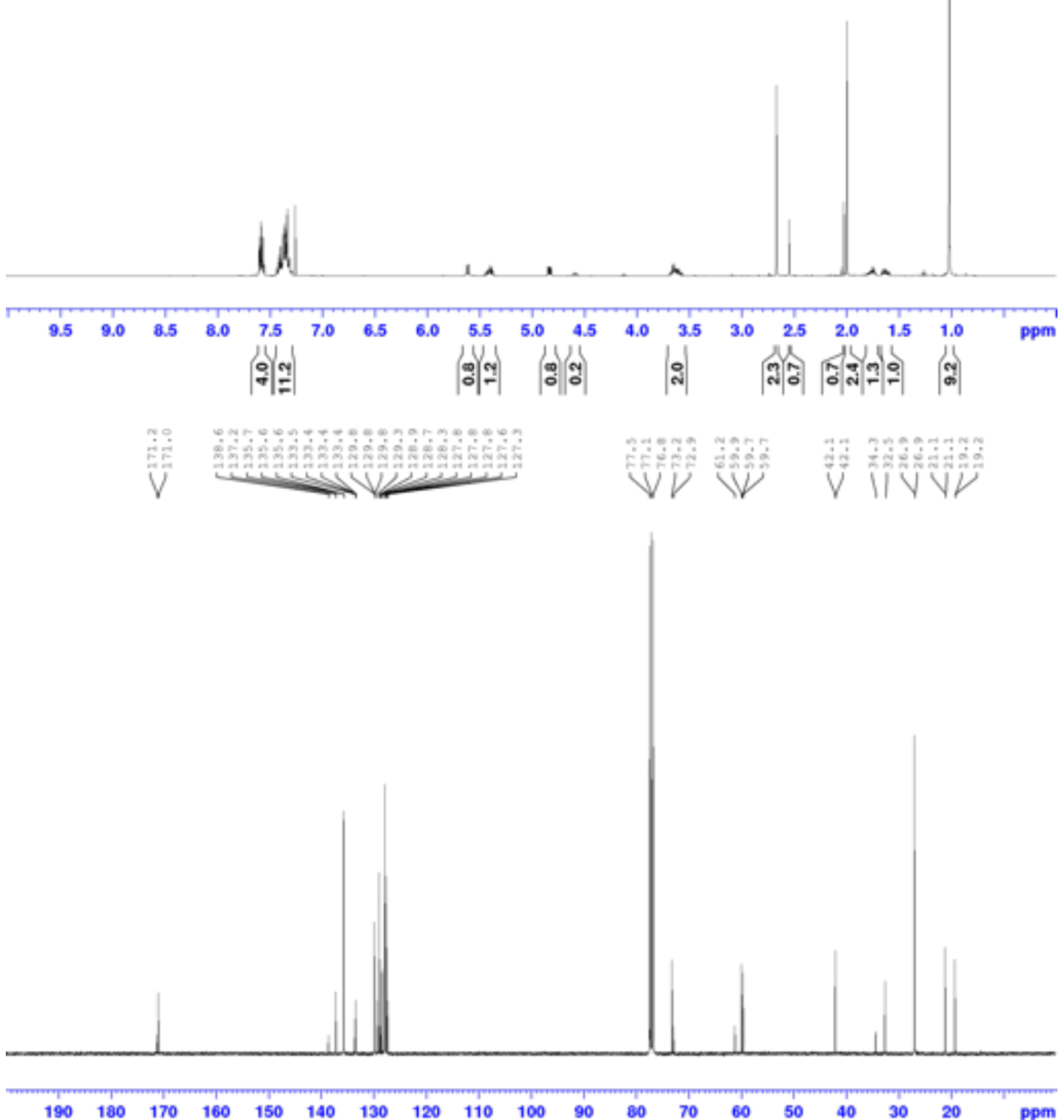
<smiles>CC(=O)OC(CCN1C(=O)c2ccccc2C1=O)[C@@H](N)c1ccccc1</smiles>

35
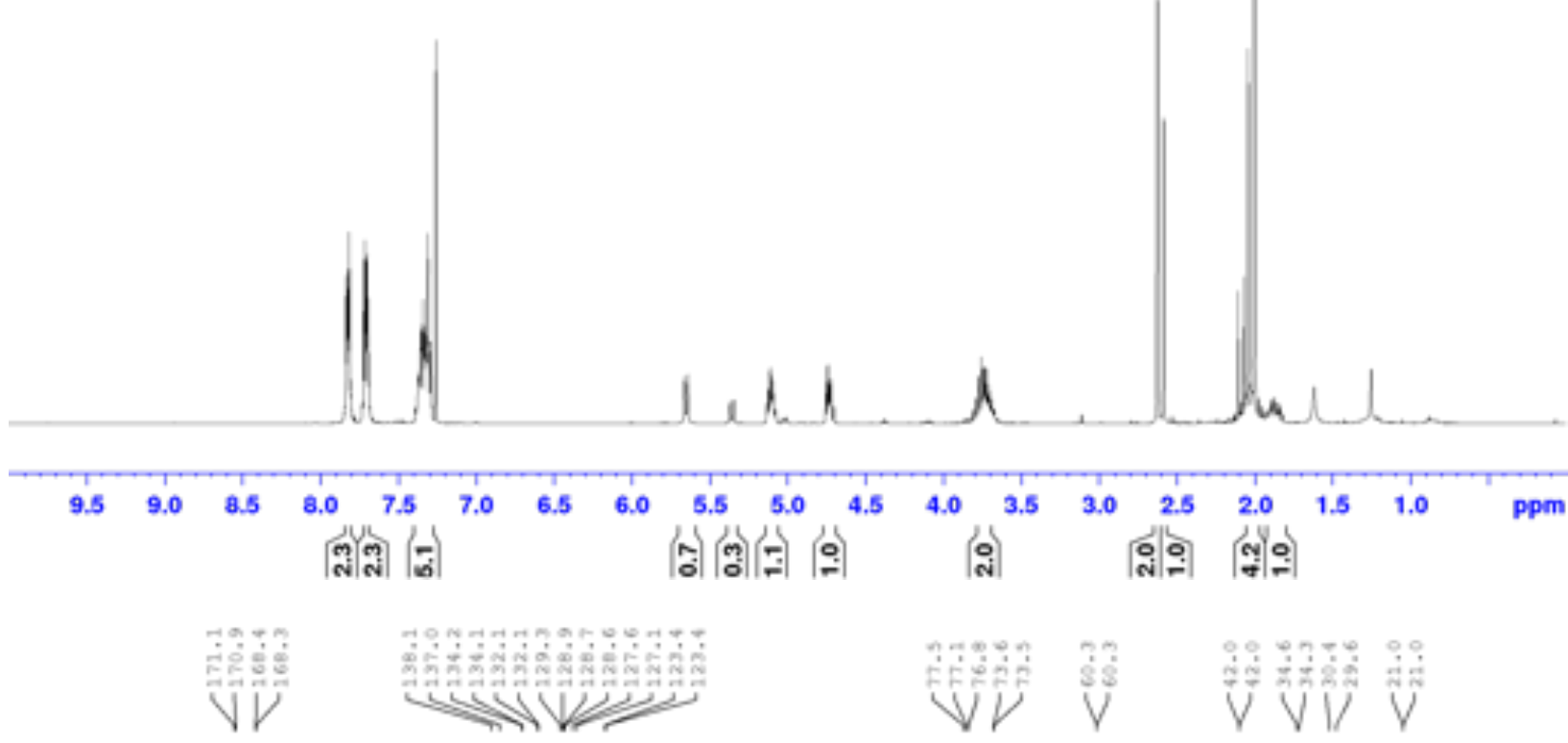

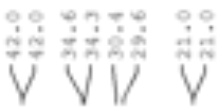

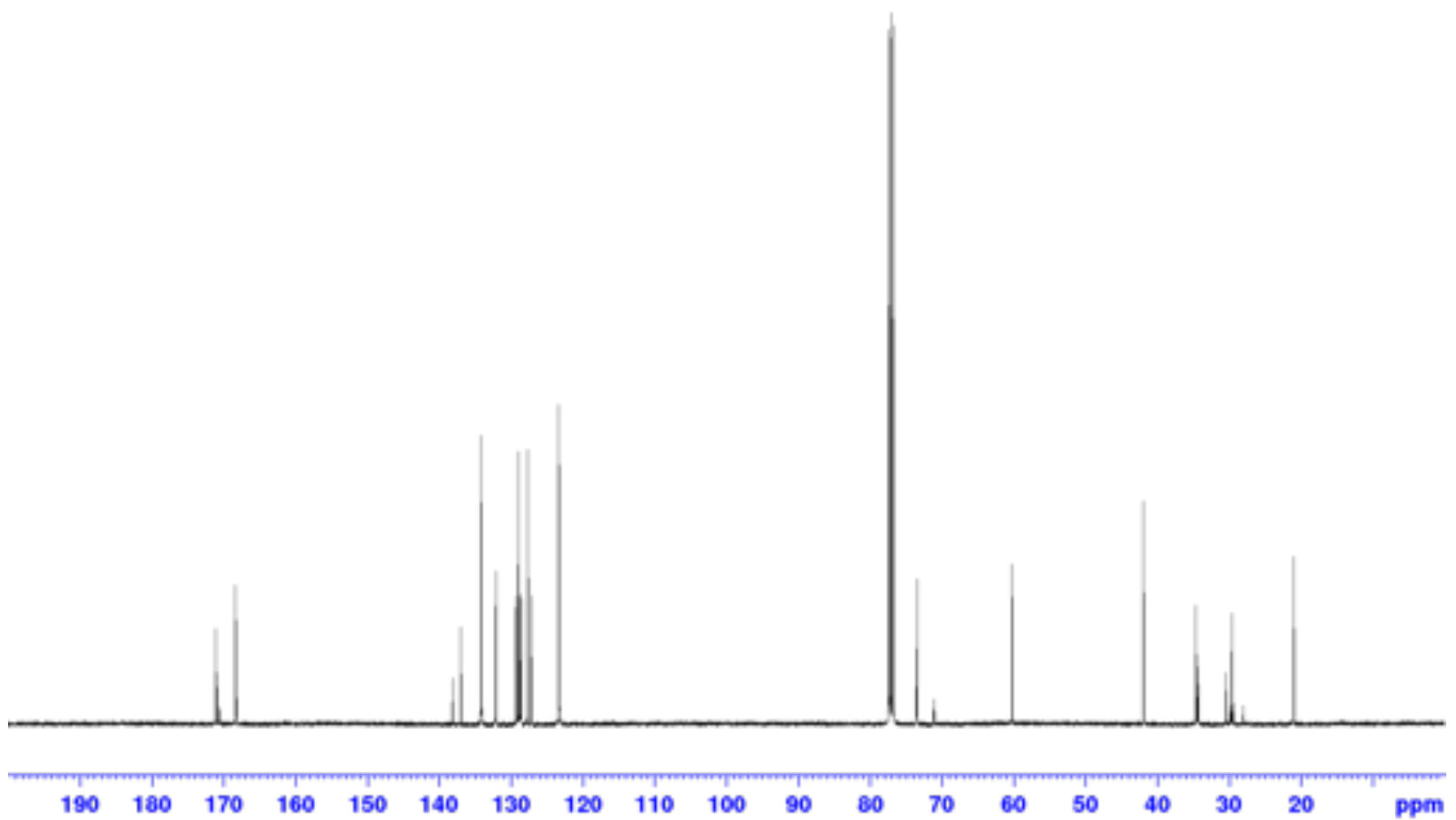




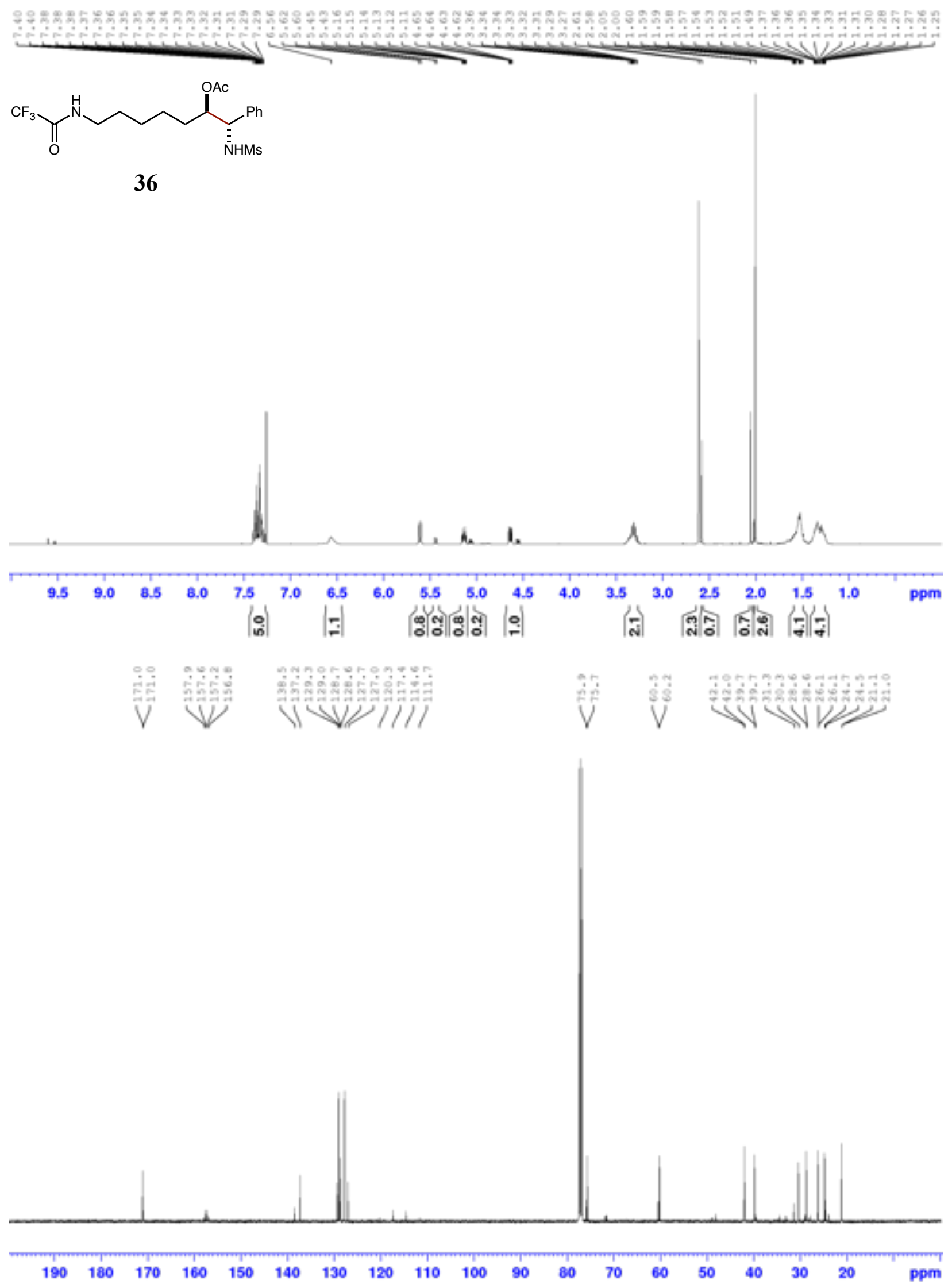




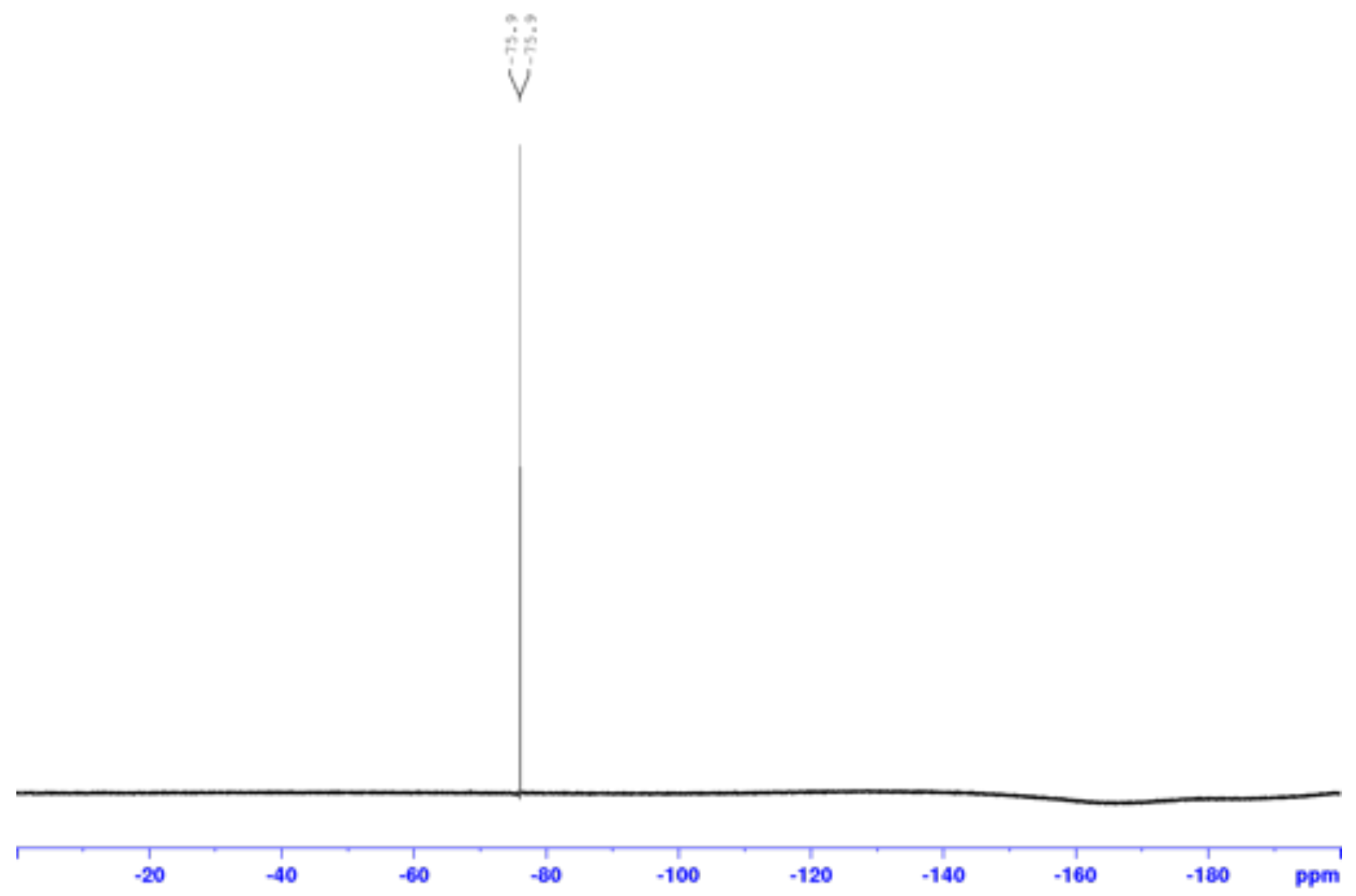


<smiles>CCCCC(OC(C)=O)[C@@H](N)CCCC</smiles>

37
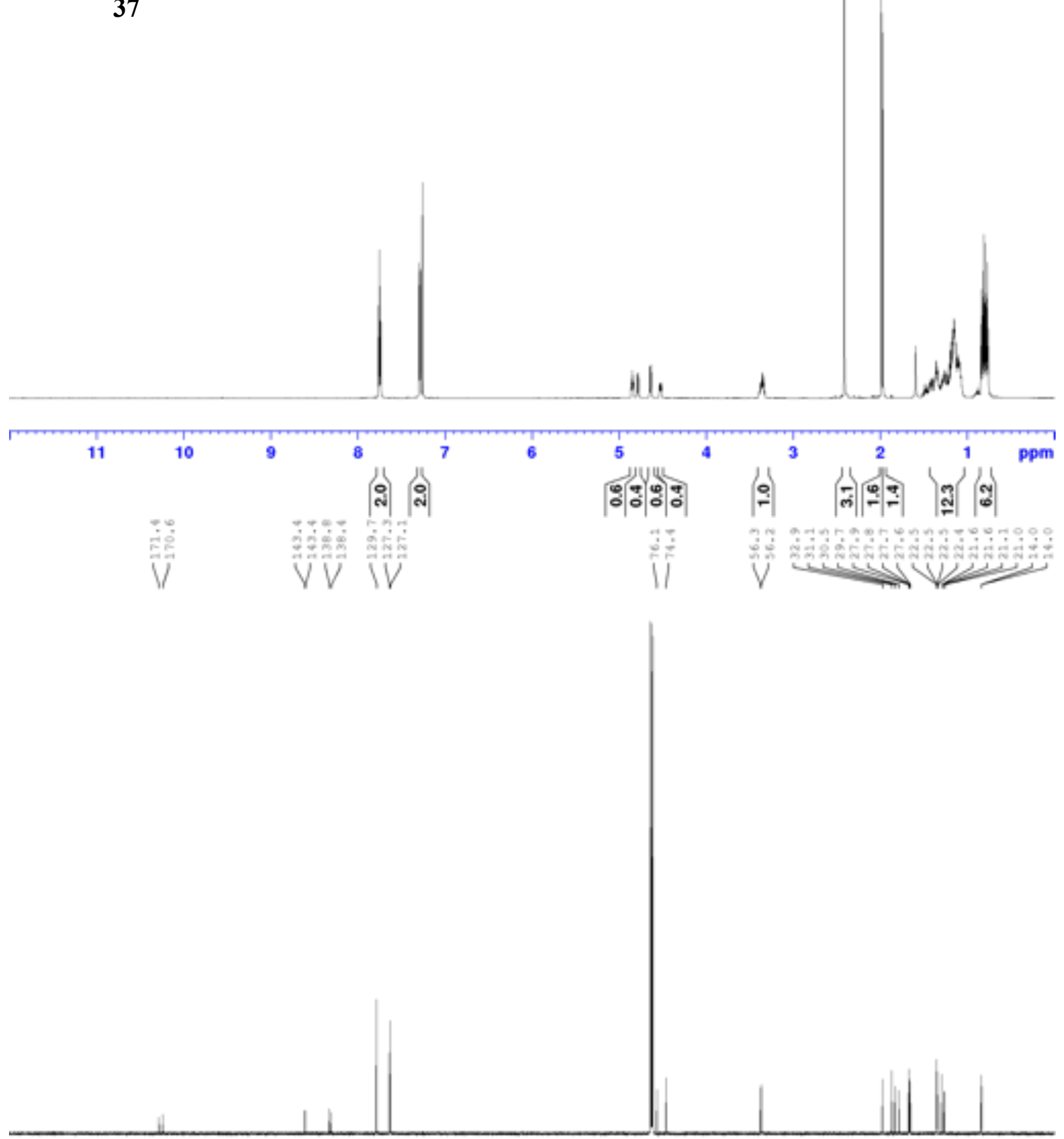

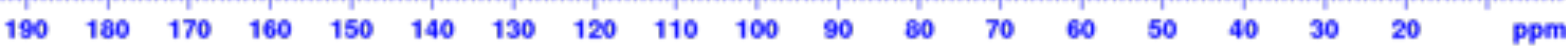



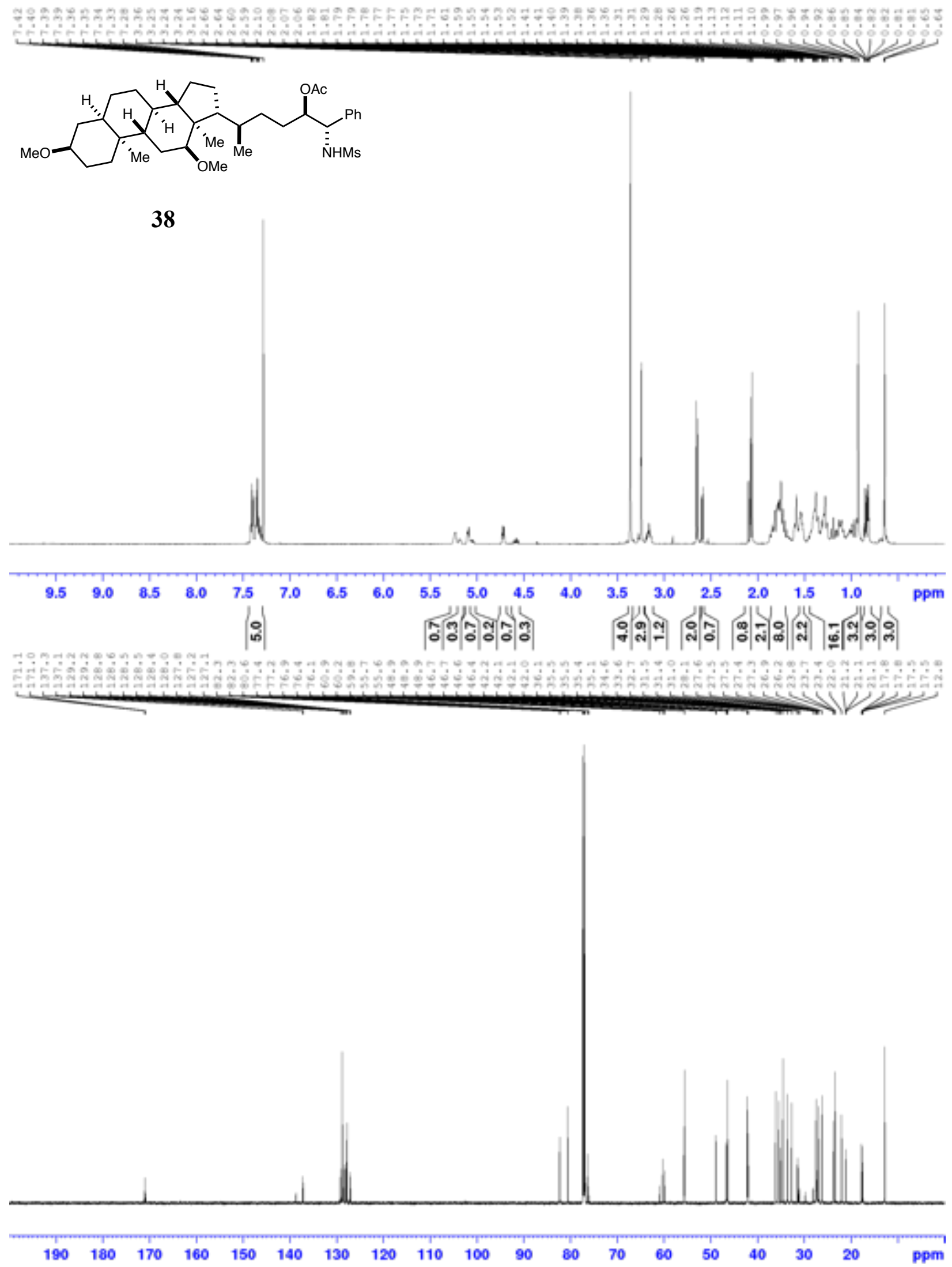
<smiles>O=S(/N=C/c1ccccc1)c1ccc(F)cc1F</smiles>
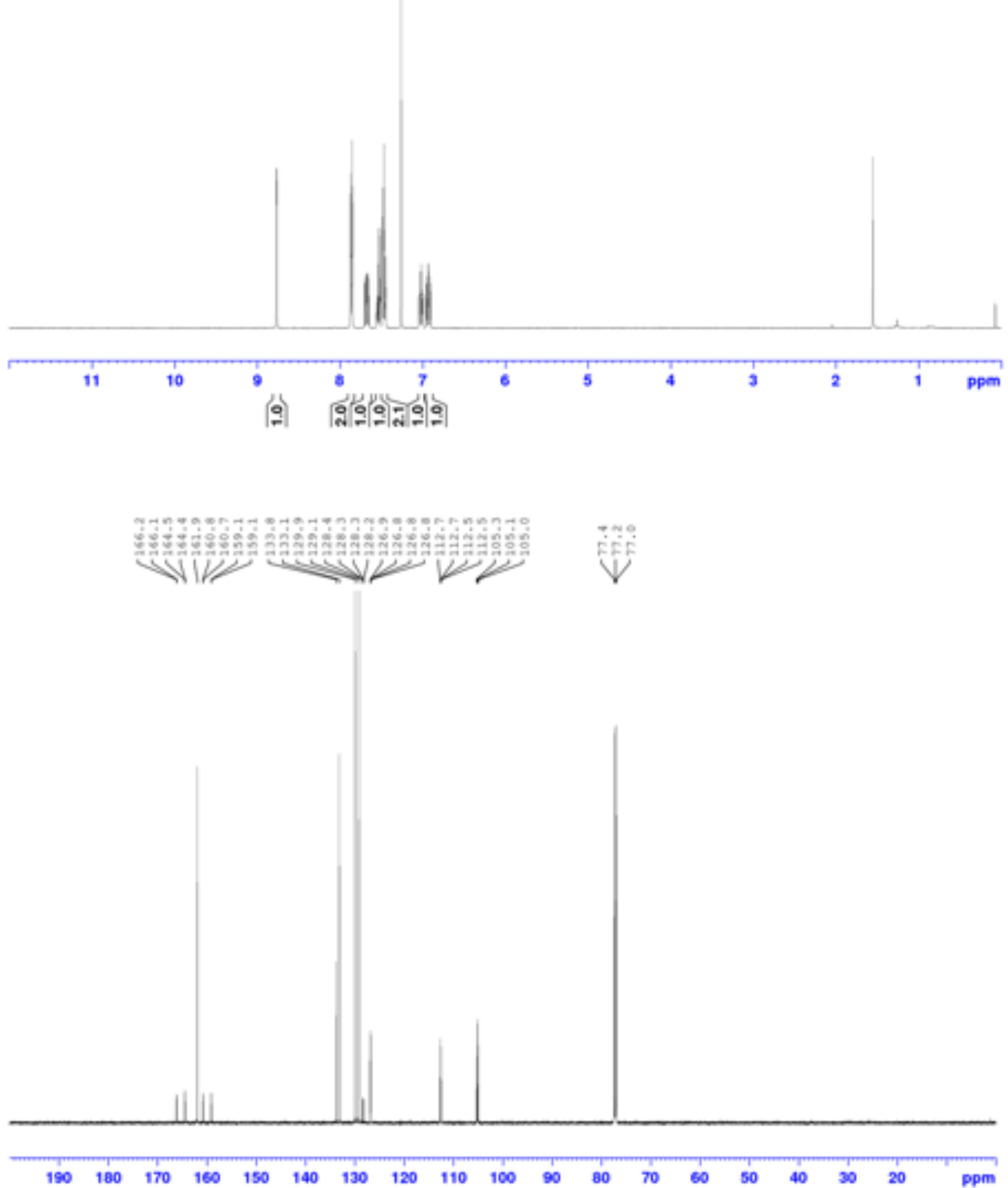

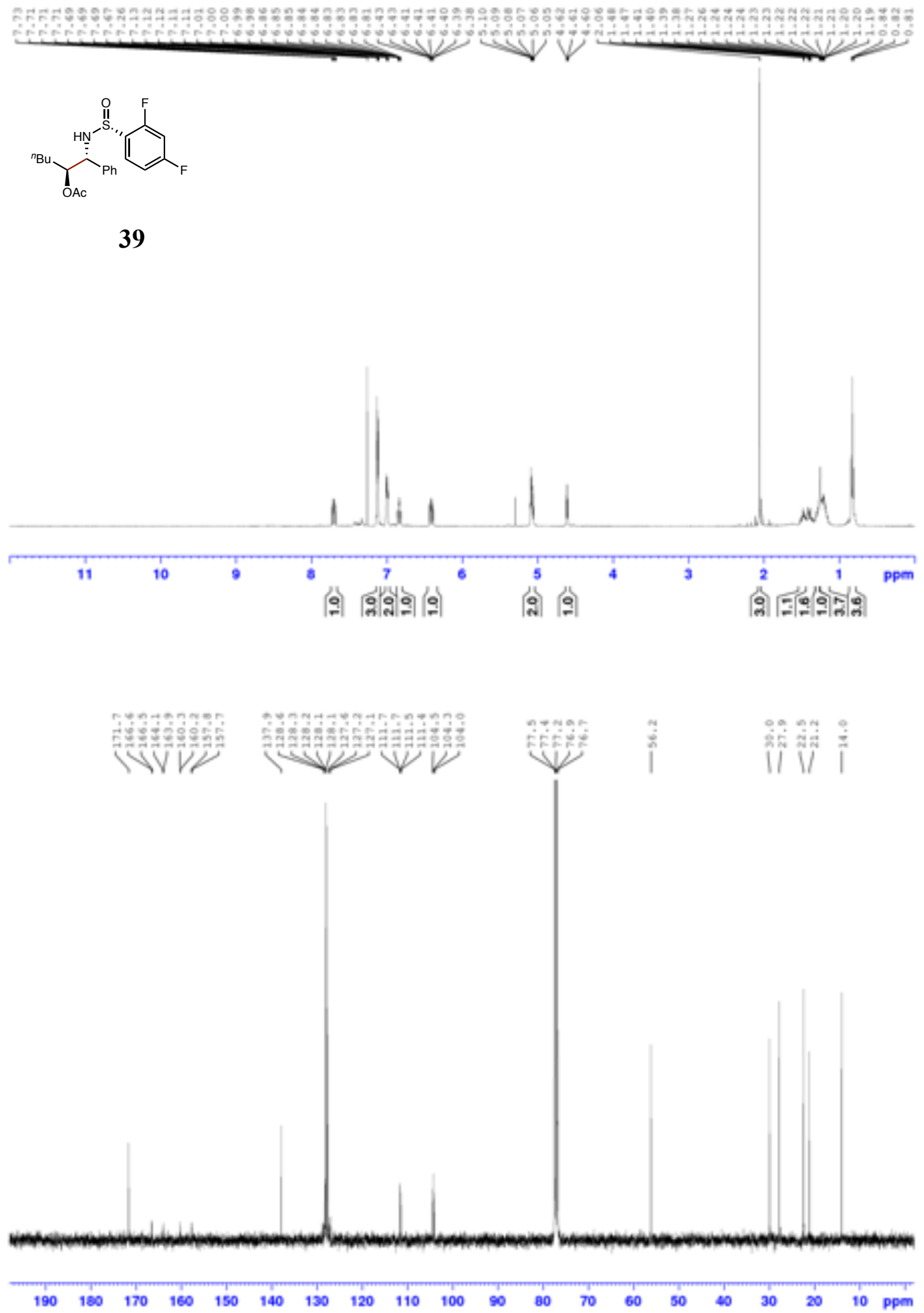
웅ํㅜ운

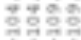

Vं

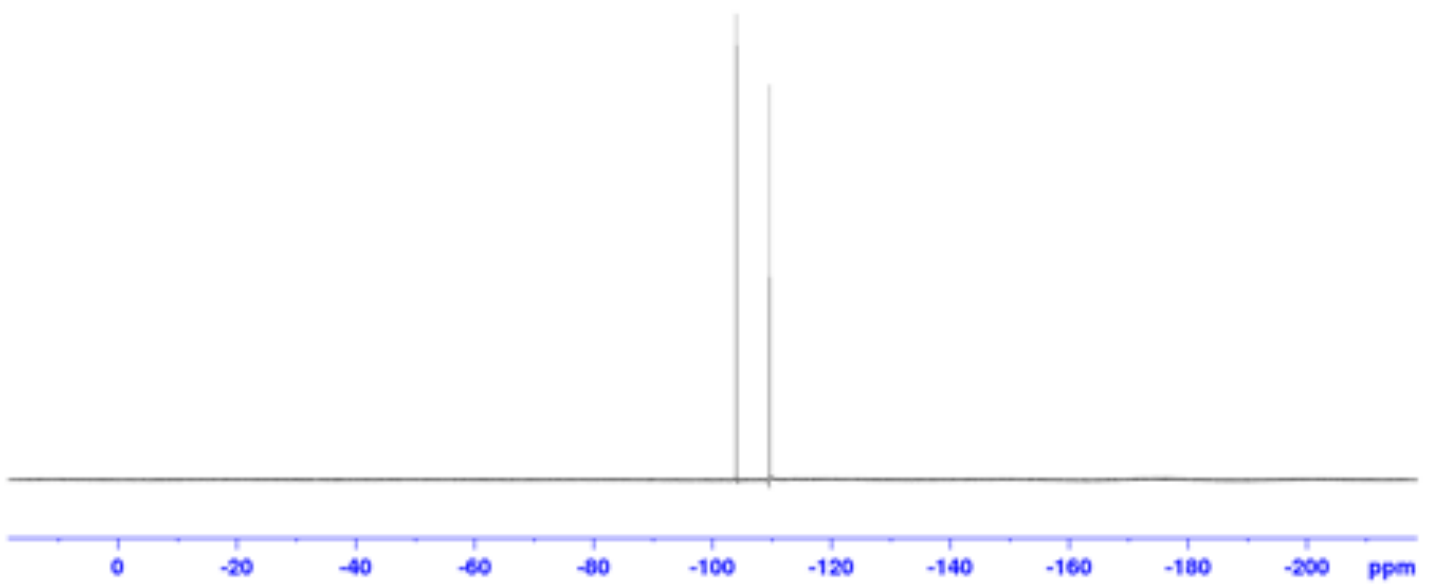




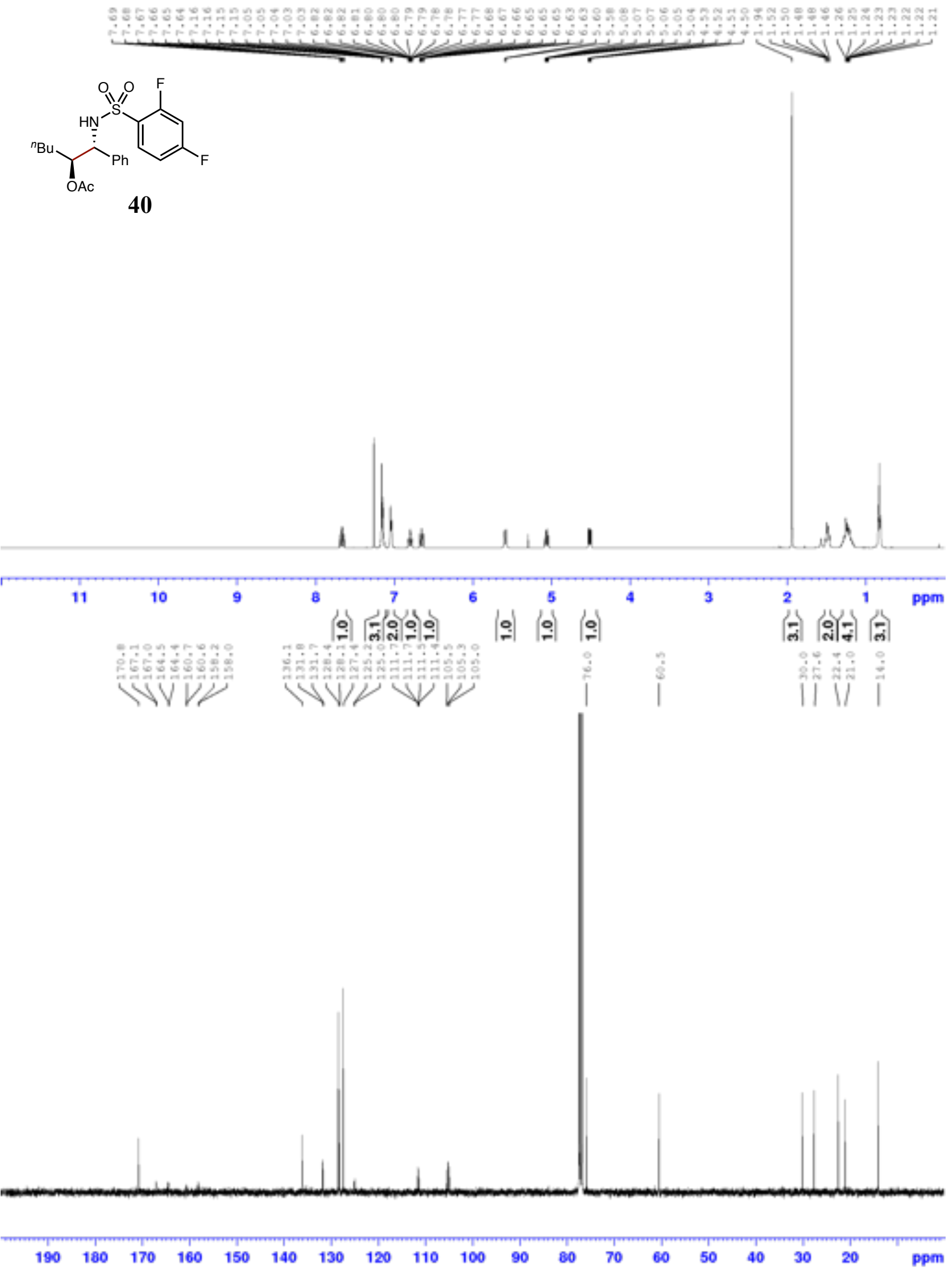




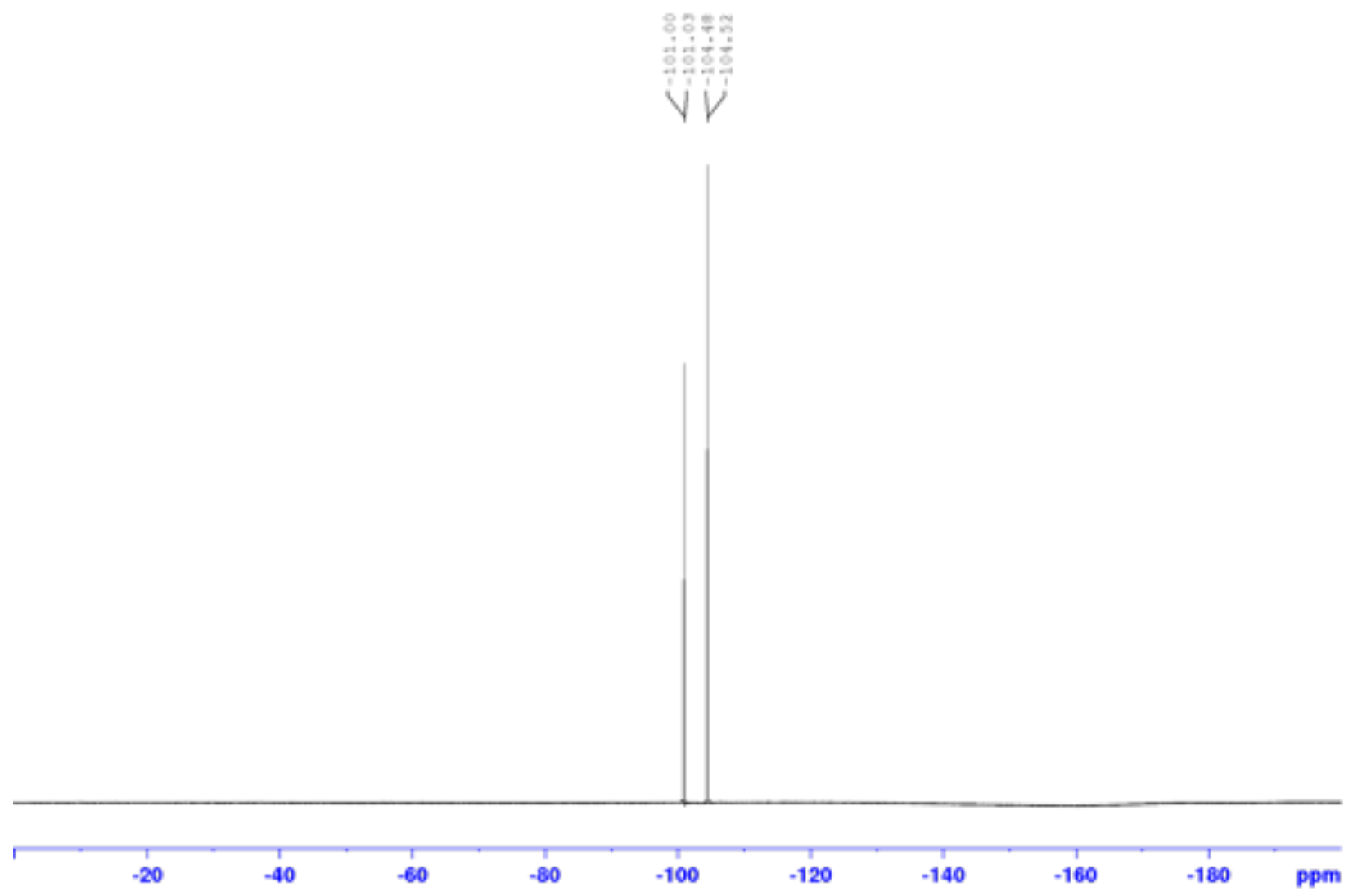


<smiles>CC(=O)OC=CC(C)C(C)(C)C</smiles>

41

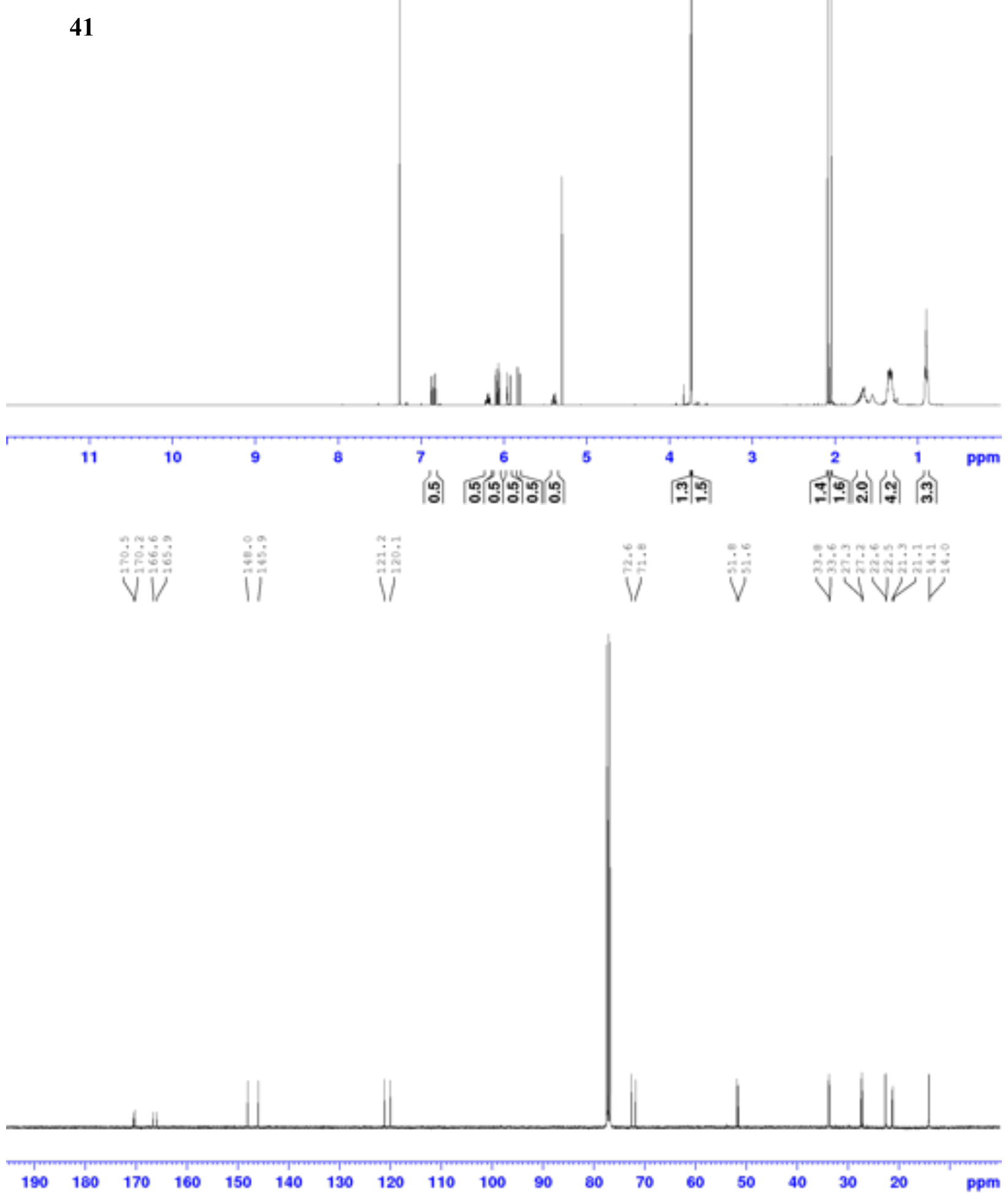




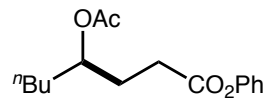

42

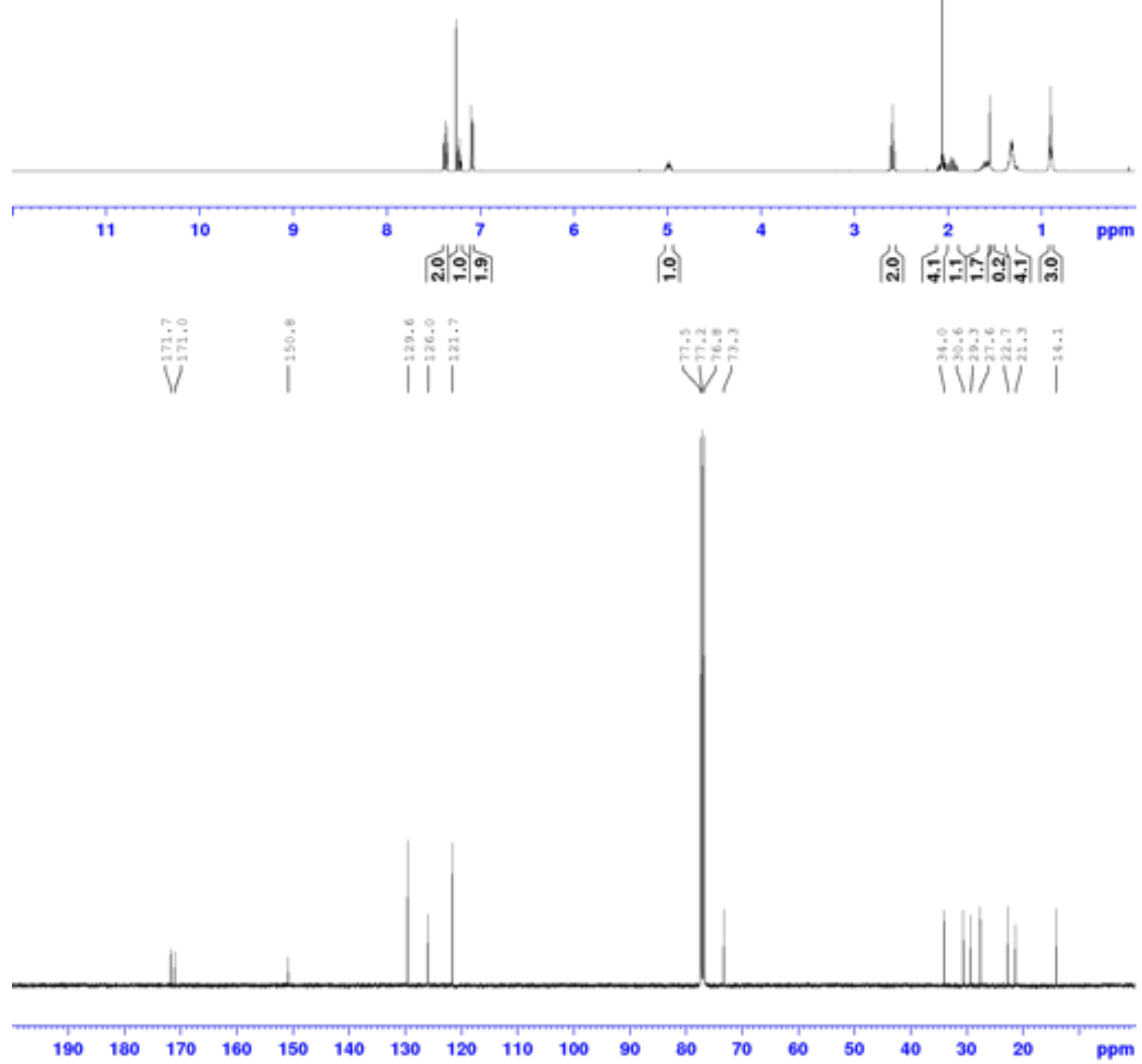




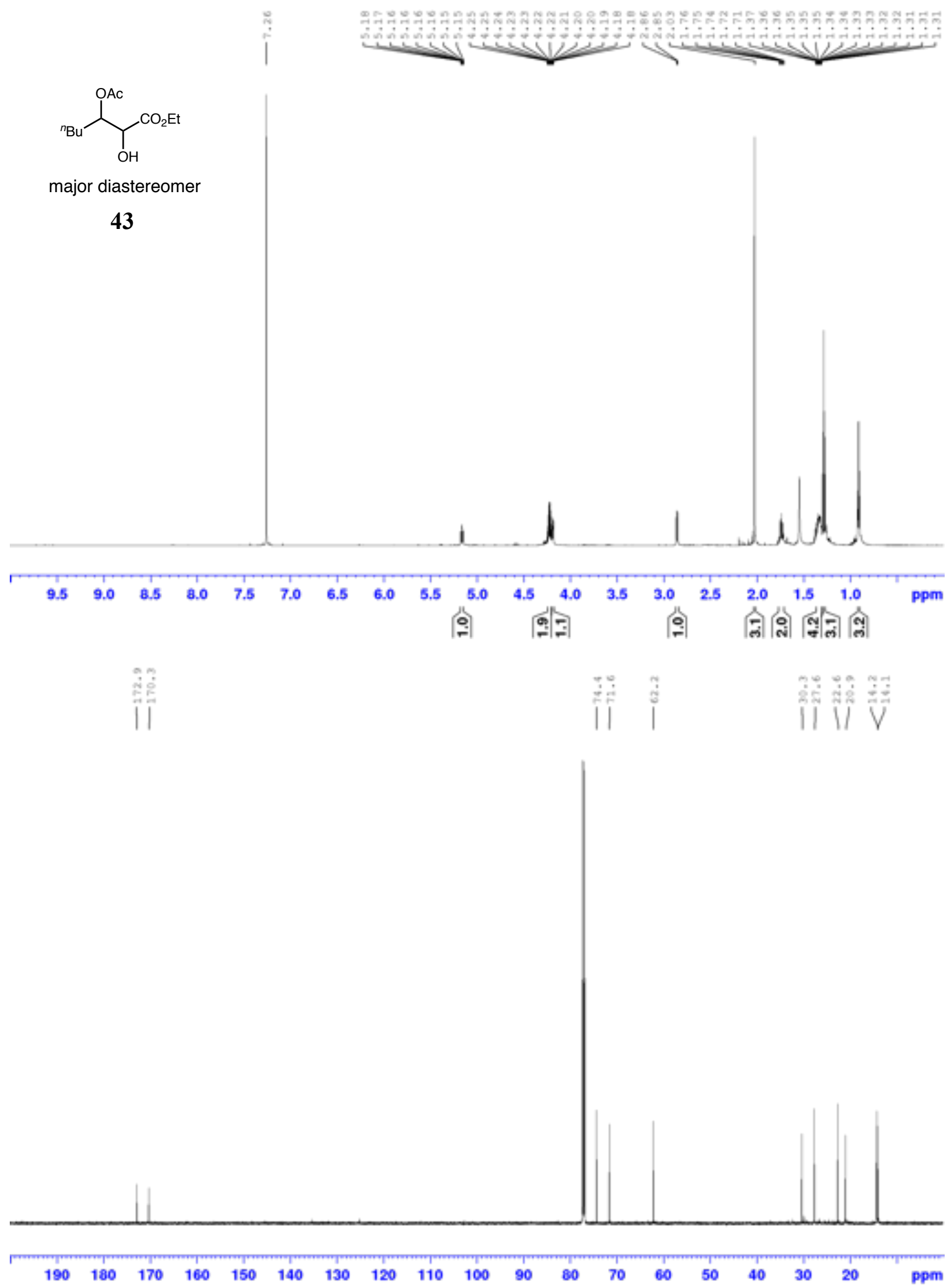



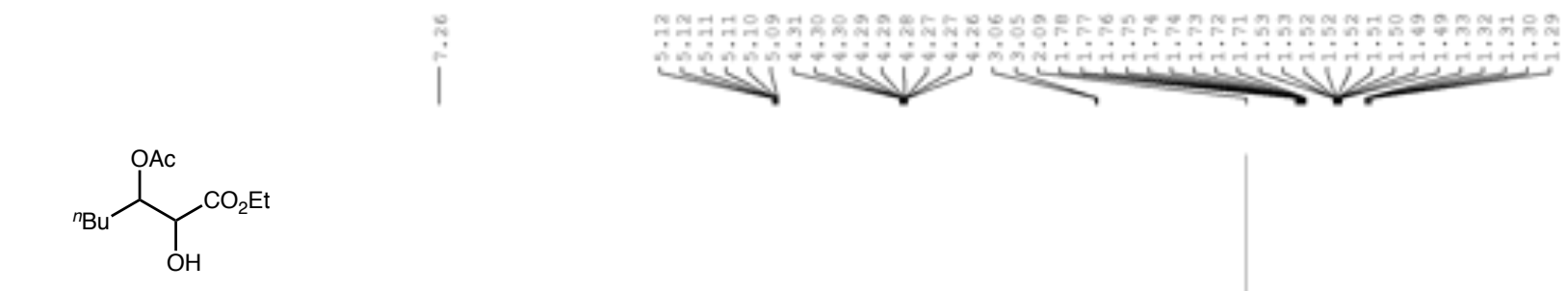

minor diastereomer

43
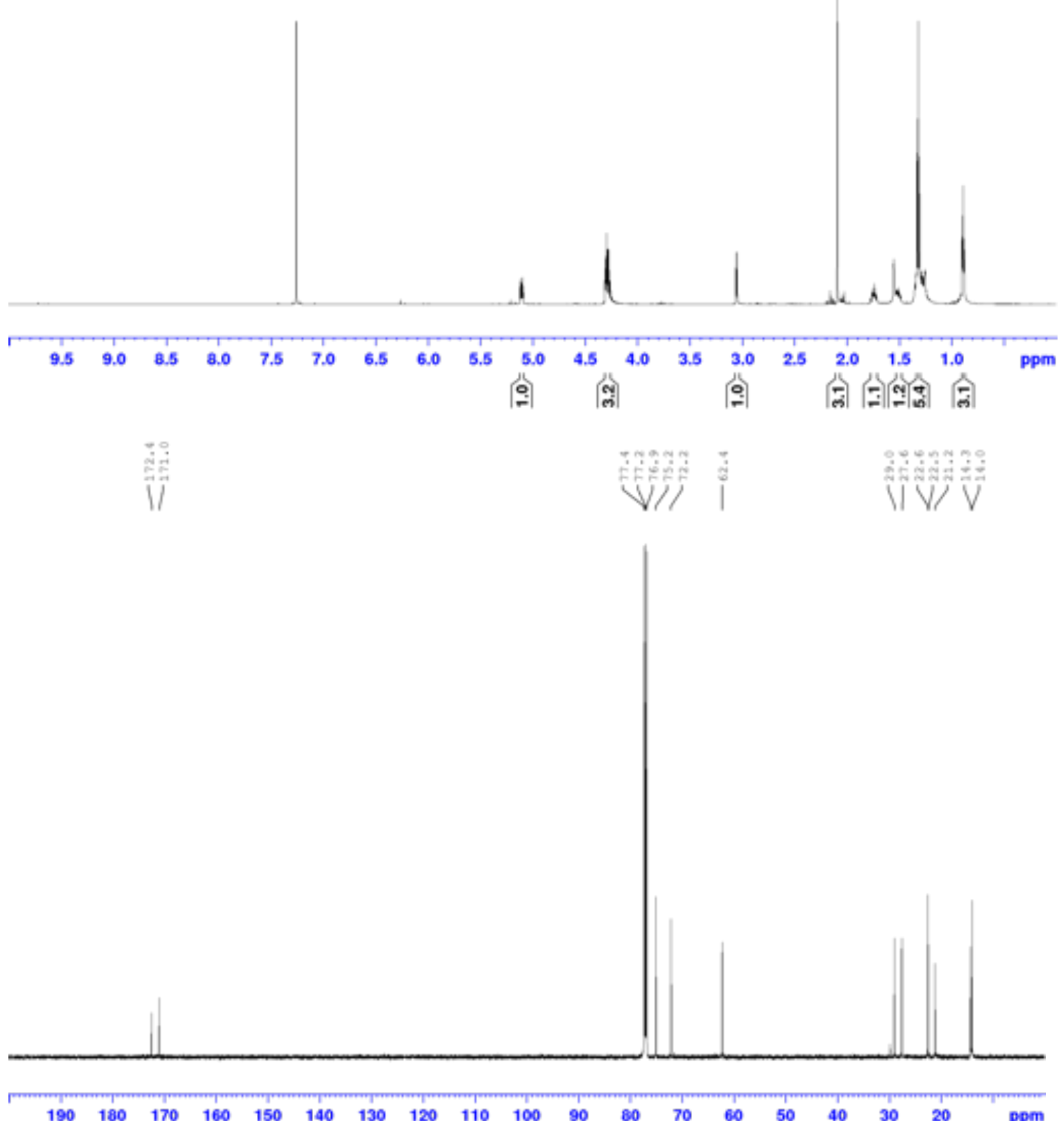


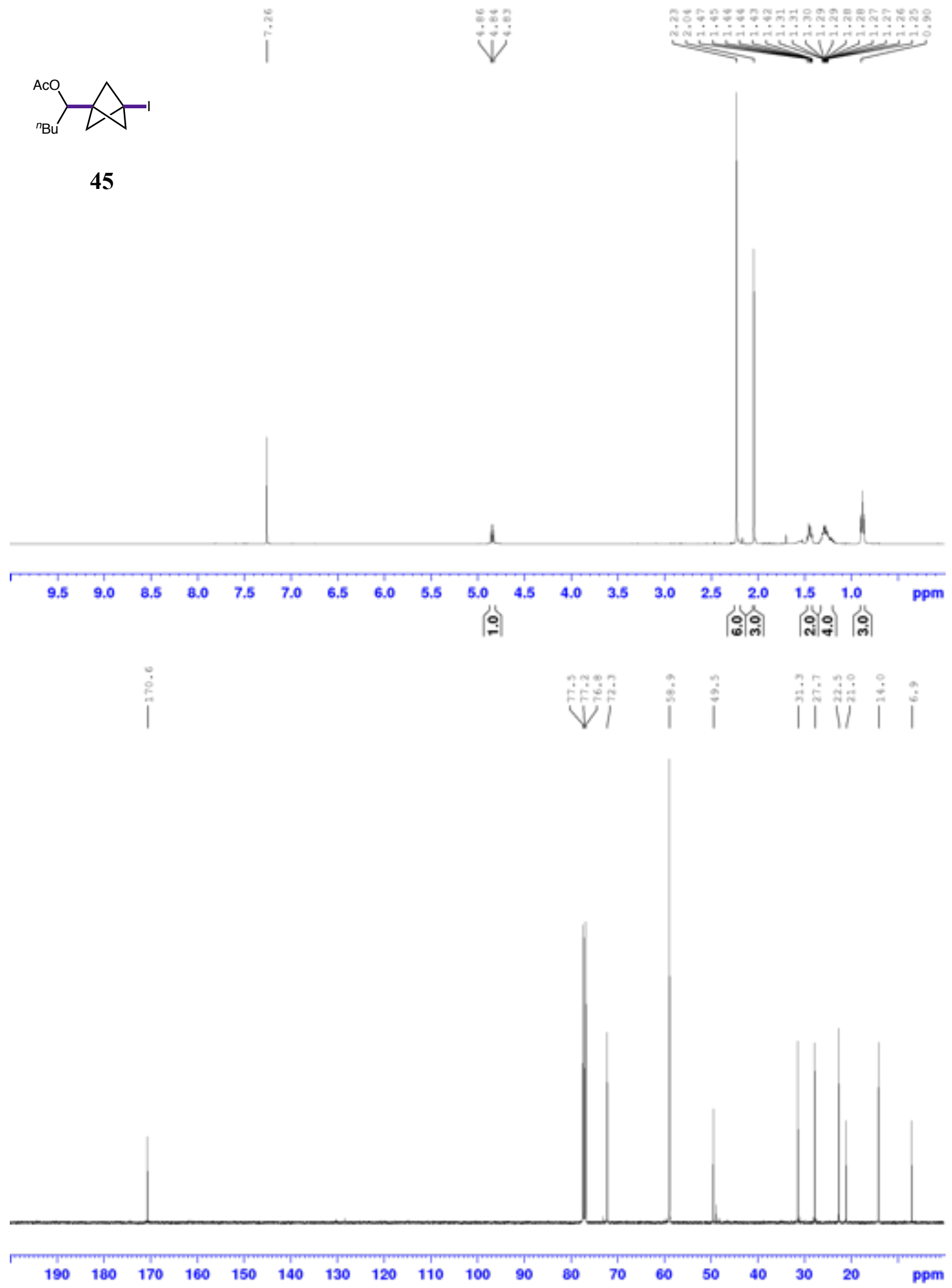

